\title{
UWB Radio Channel and Diversity Characterization for Wireless Implanted Devices
}

September 2018

Author: Carlos Andreu Estellés

Director: Dra. Concepción García Pardo

Prof. José F. Monserrat del Río

Prof. Narcís Cardona Marcet 



\section{Acknowledgements}

This thesis has been written in English. However, I would like to dedicate this part to my family and friends in my mother tongue. I apologize to the readers.

Me gustaría agradecer a mis directores de tesis Josetxo, Narcís y Conchi, todo el trabajo que han hecho junto a mi para que esta tesis haya salido adelante. Gracias Conchi por dedicar tu tiempo fuera de horario e implicarte para haber llegado hasta aquí. Una parte de mi, nunca va a salir del iTEAM. También, me gustaría agradecer a la Universitat Politècnica de València la posibilidad de disfrutar de una beca para completar mis estudios tanto en España como en Alemania.

Durante los años de la tesis, he tenido la suerte de compartir mi tiempo con un grupo de compañeros inmejorable que nunca voy a poder olvidar. Gracias a Charliet, Irene, Tere, los David's, Sandra, Alicia, Cabrejas y al resto de compañeros del MCG y de otros grupos del iTEAM. Por supuesto, a los Lord's of iTEAM con los que hemos formado una familia dentro y fuera del trabajo. Barjau, Pepelú, Gerardito, Manué, Eduardo, la historia continúa. Hay dos personas a las que me gustaría hacerles una mención especial por lo que han contribuido tanto como amigos, como para que esta tesis haya finalizado. Alex, has estado desde el primer día de una forma desinteresada ayudándome y enseñándome de que iba esto, muchas gracias germanor. Sergio, sabes que eres gran parte de este pastel. Sin tus potingues y ayuda hubiera sido imposible nada del trabajo que se ha publicado en esta tesis. Te has portado como 
un hermano para mi, muchas gracias bro. Algún día te lo recompensaré con galletas...

Me gustaría mentar a todos mis amigos que siempre han estado pendientes y apoyando para acabar esta aventura. Xuaky, Marcos, Jaume A., Jaume R., Rebeca, Blanca, Rafa y un largo etcétera, muchas gracias a todos. También, me gustaría agradecer la acogida de mis nuevos compis de Vodafone, con vosotros se hacen más cortos los largos días de trabajo en la oficina.

Ahora me gustaría agradecer el apoyo incondicional de mis dos familias. A mis padres, Jose y Mari, por siempre creer en mi, así como hacerme ver siempre el buen lado de las cosas. Gracias por apoyarme siempre. A mis abuelos, Paco y Carmina, gracias por estar siempre ahí. A mi otra familia que aprecio y quiero como si fuera la mía. Mari, Todoli, Yoli, Silvia, Chimo, Domingo, Pepe, Encarna y a todos mis sobris, os quiero mucho. Una mención a mi cuñado Emilio que hizo el primer prototipo de intestino artificial con cuatro tubos, gracias.

Noelia, gracias por apoyarme día a día. Por creer en que no hay reto que no podamos superar juntos y darme ese toque que hace que cada mañana tenga ganas de hacer las cosas mejor. Esta tesis es trabajo de los dos. Te quiero mucho.

Por último, me gustaría recordar a aquellos que, aunque no estén presentes hoy, siempre hay parte de ellos que han hecho que este trabajo haya sido posible. A mi bisabuela Carmen y a mi abuela Lola que las sigo queriendo como si estuvieran aquí. A mi abuelo Ramón, que me ha apoyado durante todo el tiempo que he llevado a cabo esta tesis y ha dejado un gran vacío recientemente. Y, por supuesto, a mi cuñada Esther. Por estar siempre presente en mí y recordarme que no hay reto que no se pueda superar. Eras, eres y serás, un ejemplo de superación y de buena persona. Te echo muchísimo de menos y te quiero cuñada. 




\section{Abstract}

Wireless Body Area Networks allow the interconnection between independent nodes located either inside or over the body skin or further. Regarding in-body communications, establishing a proper link with a capsule endoscope or with a pacemaker are examples of technological advances achieved in the last decades. In spite of these healthcare developments, current standards for these kind of communications do not allow high data rate wireless connections, which are common in the current telecommunication services. UWB systems have emerged as a potential solution for future wireless in-body communications. Nevertheless, the main drawback of UWB for in-body applications is the high attenuation of human body tissues which increases dramatically with the increment of frequency. Hence, an accurate UWB in-body channel characterization is relevant in order validate UWB frequency band as the best candidate for future networks of implantable nodes.

This thesis is devoted to test UWB technology for in-body communications from an experimental point of view. To achieve this goal, a novel spatial phantom-based measurement setup is used in several in-body propagation scenarios. Thus, the losses in the propagation medium and the channel diversity are checked in a reliable way. In order to check the values obtained in laboratory, they are compared and discussed with those obtained in an in vivo experiment. On the other hand, new UWB antenna candidates for inbody communications are designed and manufactured by using typical and new miniaturization and antenna optimization techniques for this purpose. Finally, diversity-based techniques are used to improve the performance of the propagation channel in two different in-body scenarios. 



\section{Resumen}

Las redes de área corporal permiten la interconexión de nodos independientes situados dentro o fuera de la superficie corporal o, incluso, alejados de dicha superficie. En cuanto a las comunicaciones intracorporales, el establecimiento de un enlace robusto con una cápsula endoscópica o con un marcapasos, son ejemplos de los avances tecnológicos conseguidos en las últimas décadas. A pesar de estos desarrollos en asistencia sanitaria, los estándares actuales para este tipo de comunicaciones no permiten conexiones inlámbricas de alta velocidad de transmisión, las cuales son comunes en los servicios actuales de telecomunicaciones. Los sistemas UWB han surgido como potencial candidato para las futuras redes de comunicaciones inalámbricas intracorporales. No obstante, el principal obstáculo de la tecnología UWB para aplicaciones intracorporales es la alta atenuación que sufren las señales transmitidas al atravesar los distintos tejidos corporales, que aumenta drásticamente con el aumento de la frecuencia. Por tanto, es importante una caracterización precisa del canal UWB intracorporal a la hora de validar dicha banda como la adecuada para este propósito.

Esta tesis se centra en el análisis de la tecnología UWB para posibilitar comunicaciones intracorporales inalámbricas desde un punto de vista experimental. Para conseguir este objetivo, se ha empleado un novedoso sistema de medidas experimental basado en fantomas en diversos escenarios de propagación intracorporal. De esta forma, se pueden comprobar las pérdidas de propagación en el medio así como la diversidad del canal de una forma fiable. Con el fin de validar los valores obtenidos en el laboratorio, se han comparado y analizado con los obtenidos en un experimento in vivo. Por otro lado, se han diseñado y fabricado nuevas antenas UWB candidatas para comunicaciones 
intracorporales, empleando técnicas existentes y nuevas de miniaturización y optimización. Finalmente, se han usado técnicas basadas en diversidad para mejorar el rendimiento del canal de propagación en dos escenarios intracorporales diferentes. 


\section{Resum}

Les xarxes d'àrea corporal permeten la interconnexió de nodes independents situats, o bé dins, o bé sobre la pell, o inclús allunyats del propi cos. Pel que fa a les comunicacions intracorporals, l'establiment d'un bon enllaç amb una càpsula endoscòpica o amb un marcapassos, són exemples dels avanços tecnològics aconseguits les darreres dècades. A pesar d'aquests desenvolupaments en assistència sanitària, els estàndards actuals per a aquests tipus de comunicacions no permeten connexions sense fil d'alta velocitat de transmissió, que són habituals als serveis actuals de telecomunicacions. Els sistemes UWB han sorgit com una solució potencial per a les futures comunicacions sense fil intracorporals. No obstant, el principal obstacle de la tecnologia UWB per a les aplicacions intracorporals és l'alta atenuació dels teixits del cos humà, que augmenta dràsticament amb l'increment de freqüència. Per tant, és important una caracterització acurada del canal UWB intracorporal a l'hora de validar la banda de freqüència UWB com a la millor candidata per a les futures xarxes de nodes implantats.

Aquesta tesi se centra en l'anàlisi de la tecnologia UWB per a comunicacions intracorporals des d'un punt de vista experimental. Per a aconseguir aquest objectiu s'ha emprat un sistema novedós de mesures experimentals, basat en fantomes, en diversos escenaris de propagació intracorporal. D'aquesta manera es poden comprovar les pèrdues de propagació en el medi i la diversitat del canal d'una forma fiable. Per tal d'avaluar els valors obtinguts al laboratori, s'han comparat i analitzat amb aquells obtinguts en un experiment in vivo. Per altra banda, s'han dissenyat i fabricat noves antenes UWB candidates per a comunicacions intracorporals emprant tècniques típiques i noves 
de miniaturització i optimització d'antenes per a aquest propòsit. Finalment s'han usat tècniques basades en diversitat per a millorar el rendiment del canal de propagació en dos escenaris intracorporals diferents. 




\section{Contents}

Abstract vii

$\begin{array}{lll}\text { Contents } & \text { XV }\end{array}$

1 Introduction 1

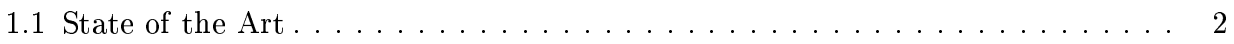

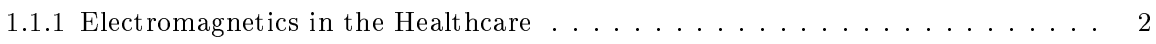

1.1.2 Standardization of Wireless Medical Systems . . . . . . . . . . . . . . . . . 4

1.1.3 Revision of Current Standard for Next Generation of In-body Devices . . . . . . 6

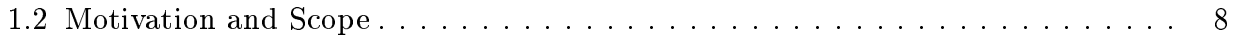

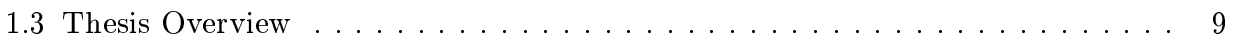

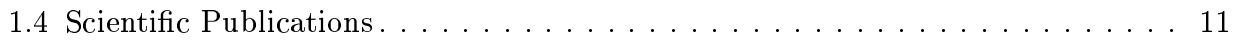

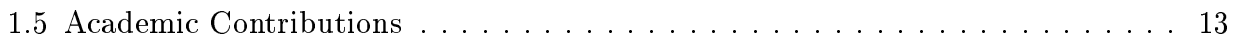

2 Experimental Measurement Setup $\quad 15$

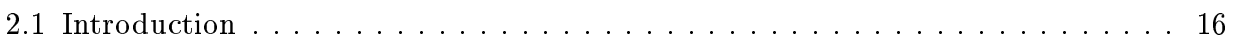

2.1.1 Reproducing the Propagation through Living Tissues . . . . . . . . . . . . 16

2.1.2 Frequency-Domain Channel Sounder . . . . . . . . . . . . . . . . 19 
2.2 Phantom-based Measurements . . . . . . . . . . . . . . . 20

2.2 .1 UWB Phantom. . . . . . . . . . . . . . . . . . . . . . . 20

2.2 .2 Measurement Setup . . . . . . . . . . . . . . . . . . . . . 23

2.3 In vivo Measurements. . . . . . . . . . . . . . . . . . . . 32

2.3 .1 Living Animal Subject . . . . . . . . . . . . . . . . . . . . . 32

2.3 .2 Measurement Setup . . . . . . . . . . . . . . . . . . . . . 32

3 UWB Biomedical Antennas 39

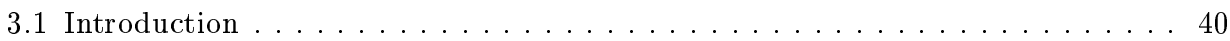

3.1 .1 Antenna Fundamentals . . . . . . . . . . . . . . . . . . . . 40

3.1 .2 Overview of UWB antennas for Biomedical Applications . . . . . . . . . . . . 44

3.2 UWB Implantable Antennas . . . . . . . . . . . . . . . . 47

3.2.1 Typical Procedure for In-Body Antenna Models . . . . . . . . . . . . . . . 47

3.2 .2 Direct Antenna Design Procedure . . . . . . . . . . . . . . . . . . . . . . . 69

3.3 UWB On-Body Antennas . . . . . . . . . . . . . . . . . . 80

3.3.1 Simulation setup \& Antenna Candidates . . . . . . . . . . . . . . . . . 82

3.3.2 Simulated Results . . . . . . . . . . . . . . . . . . . . . . . . . . 85

3.3.3 Manufacturing \& Testing . . . . . . . . . . . . . . . . . . . . . 88

4 UWB Channel Characterization $\quad 91$

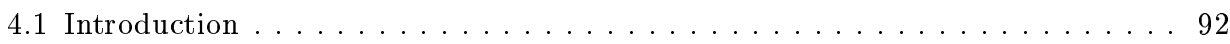

4.1 .1 Promising In-Body Scenarios at UWB Frequencies . . . . . . . . . . . . . . 92

4.1 .2 Path Loss Models . . . . . . . . . . . . . . . . . . . . . . . . . . 93

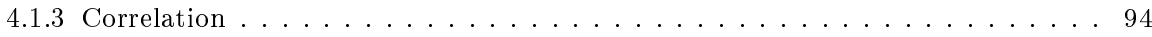

4.2 In-Body to In-Body Scenario . . . . . . . . . . . . . . . . . . . 94

4.2 .1 Preliminary Analysis . . . . . . . . . . . . . . . . . . . . . 94

4.2 .2 In-Body to In-Body Channel Characterization . . . . . . . . . . . . 96

4.3 In-Body to On-Body Scenario . . . . . . . . . . . . . . . . . . . 104

4.3.1 Path Loss Models . . . . . . . . . . . . . . . . . . . . . . . . . . . . . . . . 105

4.3 .2 Diversity of the Channel . . . . . . . . . . . . . . . . . . . . . . . . . 114 
5 Diversity Techniques

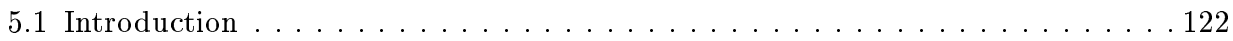

5.1 .1 Time Reversal Technique . . . . . . . . . . . . . . . . . . . . . 122

5.1 .2 Maximal-Ratio Combining. . . . . . . . . . . . . . . . . . . . . . 124

5.1.3 Reconstruction of the Channel Impulse Response. CLEAN algorithm . . . . . . 125

5.2 Time Reversal for In-Body to In-body Communications . . . . . . . . . . . . 127

5.2 .1 Configuration \& Methodology. . . . . . . . . . . . . . . . . . . . . . 127

5.2 .2 Reciprocity of the Propagation Channel _ . . . . . . . . . . . . . 128

5.2 .3 Results. . . . . . . . . . . . . . . . . . . . . . . . . . . . . . . . . . 130

5.3 MRC Technique for In-Body to On-body Communications . . . . . . . . . . 131

5.3.1 Measurement setup \& Methodology . . . . . . . . . . . . . . . . . . . . . . 131

5.3 .2 Threshold Selection for CLEAN Algorithm . . . . . . . . . . . . . . . 132

5.3.3 MRC Applied at In-body to On-body Communications . . . . . . . . . . . . 133

6 Conclusions and Perspective 137

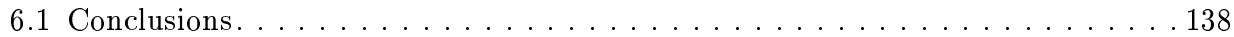

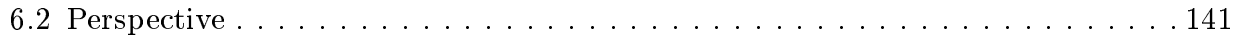

A Dielectric Properties of Human Body and Tissue-equivalent Phantoms

Bibliography 



\section{Chapter 1}

\section{Introduction}

This chapter begins with the state of the art that encompasses the main topics covered in this thesis. Besides, the motivation behind this thesis is also included. In addition, an overview of each of the chapters of this thesis is given as well. Finally, the scientific and academic contributions that have emerged from the content of this thesis are summarized. 


\subsection{State of the Art}

\subsubsection{Electromagnetics in the Healthcare}

Since 1840, when the pioneers Recamier and Pravaz experimentally proved the use of electrical current at sub-microwave frequencies to generate heat in order to destroy the uterine cancer [1], it can be stated that the scientific research and the medicine have advanced hand in hand. There are countless examples of this successful relationship. The discovery of X-ray by W.C. Roentgen implied a revolution on early diagnosis of countless pathologies. In 1952, P.M. Zoll implanted the first pacemaker in a human heart. The aforementioned advances are just a few examples of the improvements in this field through the technology.

Relevant technological steps have led to breakthrough in the medical field. In particular, the fast development in the wireless telecommunications in the last decades is a clear demonstration of this. Microwave and ultrasound technologies have led to a change in the conventional diagnostic model. They have reduced the application of invasive techniques based on ionizing radiation as well as those that use surgical procedures. Mainly, the application field of microwave-based techniques in medicine can be catalogued in three groups:

- Early diagnosis of severe diseases.

- Heat treatment at microwave frequencies.

- Monitoring techniques for patients and illnesses.

With regard to the first group, the appearance of diagnostic techniques based on microwaves almost arose as a necessity to decrease the number of invasive medical diagnostic based on surgery. For instance, the use of electromagnetic waves allows the scanning of different parts of the body wirelessly and painlessly. Nowadays, the Computed Tomography scan (CT) is an irrefutable proof of this. This complex device was mathematically posed and then designed by A.M. Cormack in 1917. Its operating principle consists in sending out a electromagnetic wave to the part of the body to be studied in order to get a $3 \mathrm{D}$ reconstruction [2]. Other relevant diagnostic techniques today in force are the Magnetic Resonance Imaging (MRI), which can detect tumor tissues invisible by X-ray techniques, or the medical ultrasound which was first used by K. Dussik to detect brain tumoral tissues recording the transmission of the sonic beam through the skull [3]. 


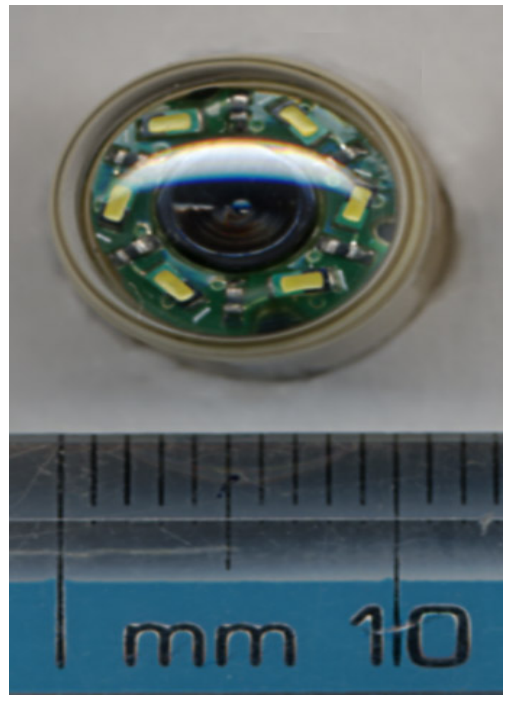

(a)

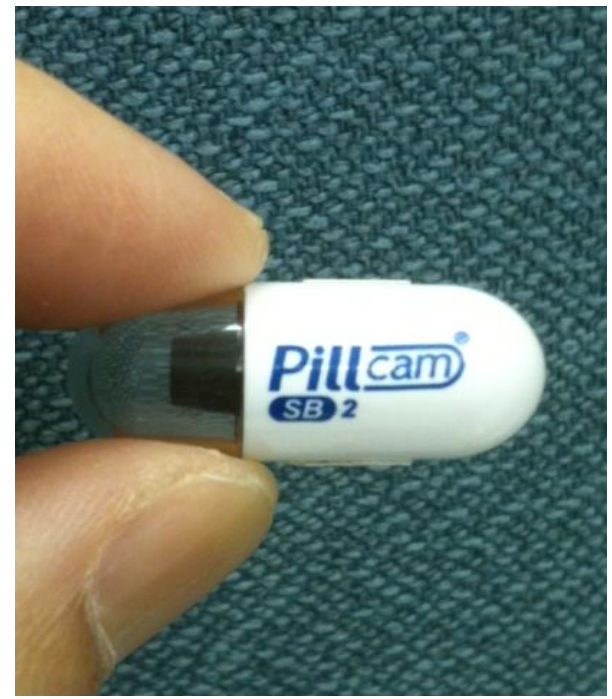

(b)

Figure 1.1: Commercial capsule endoscopy. (a) Front and (b) side view.

Most recently, the techniques based on imaging diagnosis are reaching a high degree of popularity. The most popular techniques establish wireless communications between devices outside and inside the body. In 2001, the Medtronic company (previously Given Imaging) launched into the market the first commercial model of the Wireless Capsule Endoscopy (WCE) (see Figure 1.1). This device intends to facilitate the diagnosis along the whole gastrointestinal tract and substitute the traditional endoscopy which involves a high uncomfortable procedure to the patients. Besides, the traditional endoscopy is not able to reach some areas of the small bowel due to the multiple twists and corrugations of the guts. The only way to reach some areas of the small intestine consists in using invasive techniques as laparoscopy. The operating principle of the WCE is quite simple [4]. This device collects pictures from inside the whole gastrointestinal tract reducing the patient's discomfort. The taken images are sent through the human tissues to an external reception system which is usually an on-body array of antennas connected to a Holter monitor which is in turn located on the patient's waist to record all the collected pictures. Nowadays, WCE makes easier the early diagnostic of serious illnesses such as the Chron's disease [5]. 


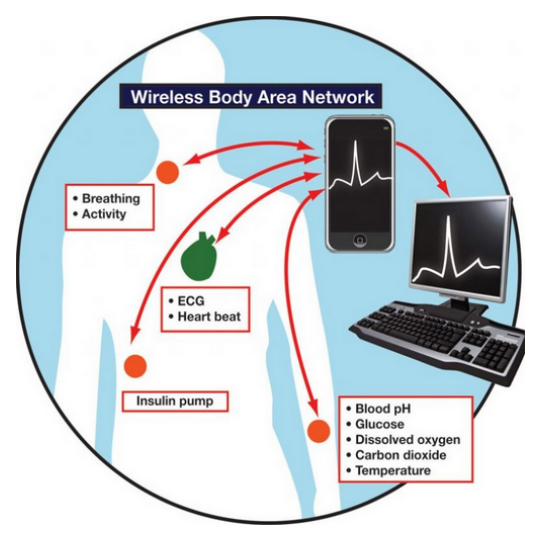

Figure 1.2: Example of multiple nodes interconnected in a WBAN network [9].

The appearance of the WCE has meant a quantitative leap forward in the radio technology applied in medicine. However, it has not been the only significant advance in this sense. Regarding the third group in the above list, many vital signs from inside the body can be monitored such as heartbeats or the electrical impulses generated by pacemakers [6]. In the view of the massive emergence of radio services for medicine, a standard became essential to coexist with other systems and technologies.

\subsubsection{Standardization of Wireless Medical Systems}

It is not before the first decade of the century $21^{\text {st }}$ when the first draft of the standard for wireless medical devices was published. The exponential growth of wireless networks along with the saturation of the radio spectrum made the standardization process necessary. For this reason, on November 2007, the LAN/MAN IEEE 802 committee formed the TG 15.6 working group in order to carry out this task [7]. Mainly, four working subgroups, composed of a committee of experts and researchers, addressed topics of the standard such as the implementation and regulation; the propagation channel model; the technique requirements and so on. Finally, the first international standard was published on February 2012 and this kind of network was called as Wireless Body Area Network (WBAN). The code of the standard, currently in force, is the IEEE Std 802.15.6 [8].

Regarding channel models, they enable and facilitate the performance assessment of the proposals for the physical layer. From these channel models, the propagation losses are characterized in both distance and operating frequency 
[10]. The TG 15.6 subgroup devoted to channel modelling established seven different propagation scenarios, named as $\mathrm{S}_{1}-\mathrm{S}_{7}$, which are determined by the location of the nodes involved in the communication. Accordingly, the nodes could be:

- Implanted, where the node is located inside the human body either beneath the skin or deeper.

- On-body, where the node is in direct contact with the skin or at least 2 $\mathrm{cm}$ away from it.

- Off-body, where the node is far $2 \mathrm{~cm}$ away from the body surface up to a maximum value of 5 meters.

The standard also defines several application scenario. Particularly, for implanted communications the $\mathrm{S}_{1}, \mathrm{~S}_{2}$ and $\mathrm{S}_{3}$ are defined as:

- $S_{1} \Rightarrow$ Communication among implanted nodes. In literature, this kind of scenario is also known as In-Body to In-Body (IB2IB).

- $S_{2} \Rightarrow$ Communication among implanted and on-body nodes. In literature, this kind of scenario is also known as In-Body to On-Body (IB2OB).

- $S_{3} \Rightarrow$ Communication among implanted and external nodes far from the body surface at least $2 \mathrm{~cm}$. In literature, this kind of scenario is also known as In-Body to Off-Body (IB2OFF).

The TG 15.6 working group set three different channel models according to the considered scenario $\left(\mathrm{CM}_{1}, \mathrm{CM}_{2}\right.$ and $\left.\mathrm{CM}_{3}\right)$. $\mathrm{CM}_{1}$ was allocated to $\mathrm{S}_{1}$, whereas $\mathrm{CM}_{2}$ was to $\mathrm{S}_{2}$ and $\mathrm{S}_{3}$. With regard to $\mathrm{CM}_{1}$ and $\mathrm{CM}_{2}$, different organizations, such as the Federal Communications Commission (FCC), decided that the Medical Implant Communication System (MICS) band, which covers a bandwidth from 402 to $405 \mathrm{MHz}$, was chosen for both channel models. MICS band was distributed in subbands up to $300 \mathrm{kHz}$. In terms of the radio performance of this band, it has a relative good wave propagation conditions through the human body tissues [11]. This is a high relevant factor since the losses suffered by the radio waves through the different tissues considerably increase as the working frequency grows as well [12]. In fact, this was a significant factor. Robustness and reliability were rewarded over other features. 


\subsubsection{Revision of Current Standard for Next Generation of In-body Devices}

The growth in the performance of wireless communication systems has been exponential within the last two decades. Principally, this higher performance has come along with the increment of the used bandwidth for current systems which reach transmission data rates of the order of $\mathrm{MHz}$ and even $\mathrm{GHz}$. In this sense, wireless medical services have suffered a stagnation.

Although new mobile and wireless communication standards encourage the use of large bandwidths to improve current applications, medical standard has not been changed since its first publication. This fact restrains the IEEE Std. 802.15.6 to allow high data rate connections, which are achieved by other telecommunication services today in force. MICS band is constrained to the use of narrow band systems up to $3 \mathrm{MHz}$. Therefore, future revisions of the current standard for in-body communications aims to study other frequency ranges to improve the performance of the current systems. Thus, new medical applications could appear in order to enhance the healthcare and the wellbeing qualitatively.

A large number of researchers and experts of this field has focused on researching new frequency bands for in-body communications as alternative of the current one. One of the proposed frequency bands has been the Industrial, Scientific and Medical (ISM) radio band which covers from 2400 to 2453.5 $\mathrm{MHz}$ [13]-[17]. However, these multipurpose band is widely used for WLAN and WPAN networks so that interferences between services could be produced. Besides, communications at this frequency band are not able to reach the data rates provided by current wireless technologies [18].

Over the last decade, the Ultra-Wide band (UWB) frequency band has been considered for short-range, high data rate communications [19]. As the European commission pointed out, this technology can provide a host of communications, measurement, location, medical, surveillance and imaging applications of benefit to various Community policies [20]. UWB technology was defined by the FCC as any wireless system which occupies a bandwidth of $500 \mathrm{MHz}$ or more (absolute bandwidth) or a fractional bandwidth larger than the $20 \%$ of the carrier frequency (relative bandwidth) [21]. The FCC approved the deployment on an unlicensed portion of the electromagnetic spectrum which covers from 3.1 to $10.6 \mathrm{GHz}$ (see Figure 1.3). The European Telecommunications Standards Institute (ETSI) defined the maximum mean power spectral density within the operating bandwidth [22]. Among the main benefits of UWB technology are the large available bandwidth, the lower power consumption, 


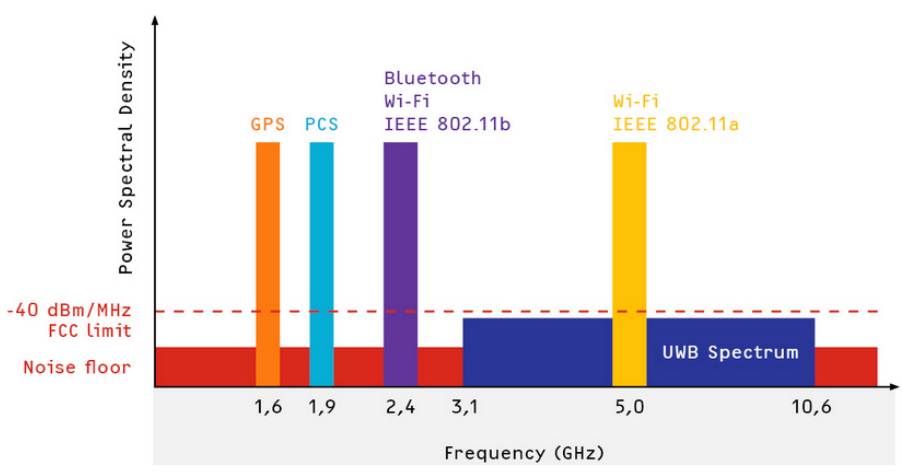

Figure 1.3: UWB frequency spectrum [24].

the low-cost of its transceivers, the simplicity of its receivers and the low profile of its antennas [23].

Concerning WBAN networks, UWB technology has been used for extracorporeal scenarios, i.e., when all the involved nodes are located outside the body. However, many research works have put UWB systems forward as a future candidate for the revision of the current standard for in-body communications [25]-[28]. In literature, it is possible to find works devoted to novel devices which benefit from this technology. For instance, cortical implants [28], UWB capsule endoscopy [29] or even innovative cancer detection techniques [30][32]. In fact, the current capsule endoscopy can take a qualitative leap forward by using UWB technology. Capsule endoscope sensors require at least 73.8 Mbps $(640 \times 480 \times 24$-bits $\times 10$-frames $/ \mathrm{s})$ to achieve high resolution images and real-time video [33]. Using UWB systems, this capacity can be achieved since UWB radio links can support up to $100 \mathrm{Mbps}$ in short-range implant communications [34]. Besides, new applications could appear such as high accurate localization and tracking of in-body devices [35] or remote drug delivery and biopsy extraction [36].

The main drawback of UWB systems for in-body communications is the large attenuation suffered by UWB signals through human body tissues [37]. In addition, this attenuation increases with the increment of frequency since the human body tissues are frequency-dependent [12]. Hence, a large number of validation must be performed to consider UWB technology as the best candidate for this purpose. 


\subsection{Motivation and Scope}

The international standard for WBANs (IEEE 802.15.6) establishes the basis for in-body communications. MICS band is the frequency band used when at least one involved node is implanted inside the human body. Even though this band has a good wave propagation conditions and certain robustness, it is mainly limited in terms of bandwidth.

UWB frequency band has emerged as a potential candidate for the revision standard of in-body communications. Some studies point out how the losses in the propagation medium increases dramatically with increments of frequency [38]. Therefore, many more studies should be performed to validate this band as the best choice. In addition, these losses could be compensated by means of other techniques which profit from the benefits of UWB systems.

On the other hand, UWB in-body channel characterization is challenging. The human experimentation is practically forbidden for obvious moral and physical integrity reasons. As alternatives to channel characterization, there are mainly two options:

- Mathematically, by using complex anatomical models embedded in an electromagnetic computer software.

- Experimentally, either by laboratory tests or by using living animals on in vivo experiments.

In both cases, transmitters and receivers involved in the communication must work efficiently taking into account the propagation medium around them.

With regard to experimental characterization, the availability of surgical rooms and the medical staff to perform in vivo measurements is limited. Nonetheless, validation by using values of living tissues is practically mandatory. In order to make accurate measurements in laboratory tests, the measurement setup is high relevant. The element which would replace the human tissues has to be able to reproduce the dielectric properties of these tissues with high accuracy to obtain realistic values. In literature, chemical compounds known as phantoms try to imitate this properties at certain frequencies [39], [40]. However, it is practically impossible to find phantoms which imitate the complex permittivity of human tissues within a large bandwidth.

This thesis is focused on UWB channel characterization from an experimental point of view. To achieve this goal, firstly new UWB antennas are designed 
and miniaturized to work in this medium efficiently. On the one hand, a high accurate phantom, which achieves the best approximation of complex permittivity of the human muscle tissue within UWB so far, is used to perform massive measurements. Besides, diversity techniques to enhance the channel performance are tested from these measurement campaigns. On the other hand, the phantom-based values are thoroughly compared and discussed with in vivo measurements performed in a surgical room by using a living animal.

The main goals to be attained in this thesis can be summarized as follows:

1. Design and manufacture UWB antennas to be implanted as well as to work over the skin surface. Testing the most popular design procedures and evaluating new methods of antenna miniaturization and optimization.

2. Development of a new measurement setup to perform high accurate experimental measurements.

3. Study the UWB propagation channel in several propagation scenarios by laboratory tests and in vivo measurements. Discussion of the results.

4. Assessment of the impact of diversity techniques such as Time-reversal or Maximal ratio Combining on the in-body channel performance.

\subsection{Thesis Overview}

The organization and content of each chapter of this thesis is summarized below.

Chapter 2 describes the novel measurement setup developed for this thesis. It explains the set-up of every pieces of equipment used to perform experimental channel measurements. For experimental measurements in laboratory facilities, a new accurate UWB phantom is presented. For both phantombased and in vivo measurements, two different software tools are programmed in order to control and synchronize all the devices involved in. Both software tools are described throughout this chapter. In this thesis, two phantom-based IB2IB and IB2OB scenarios are considered to recreate the propagation through human tissues. Both scenarios are detailed as well. Finally, the measurement setup and methodology for in vivo experiments are shown.

In Chapter 3, new UWB antennas for biomedical applications are designed, miniaturized and optimized. On the one hand, a new discone monopole antenna is designed by using the most popular miniaturization procedure for 
implantable antennas. On the other hand, a well-known UWB monopole antenna is miniaturized and optimized using a different approach. A CPW-fed antenna is optimized taking into account the dielectric properties of the human tissue from the first design stage. Thus, it is tried to keep the features that this antenna has in free space. The impact of this new approach in channel performance is assessed as well. This optimization technique is also used to design and evaluate new slot antennas as on-body receivers.

Chapter 4 is focused on UWB channel characterization by phantom-based and in vivo measurements. The pieces of equipment, software tools and the methodologies explained in Chapter 2 are used throughout this chapter. Results are divided considering the application scenario (IB2IB and IB2OB). For both of them, a large number of measurements are obtained by phantom-based setups. The path loss is obtained and the best approximation model is assessed. Besides, a thorough comparison with other related works of literature is carried out. The diversity of the channel is also evaluated from a experimental point of view in both scenarios.

In the IB2IB setup, the effect of locating the transmitter and receiver at different heights in channel performance is studied. The correlation is tested in transmission and reception.

In the IB2OB scenario, the losses and diversity are assessed for the whole available bandwidth as well as for subbands of $500 \mathrm{MHz}$ in order to evaluate the frequency dependence of UWB in-body radio channel characteristics. Furthermore, the differences in channel performance by using different on-body receivers are evaluated and discussed. Finally, a comparison between phantombased and in vivo measurements is provided.

Chapter $\mathbf{5}$ is devoted to apply methods based on diversity techniques to enhance the channel performance. On the one hand, the Time-reversal signal processing is used in a phantom-based IB2IB scenario. As previous step, the reciprocity of the channel is evaluated. On the other hand, the maximal ratio combining technique is applied in an IB2OB scenario by using several receivers. The channel impulse response is reconstructed from the received signal and the transmitted pulse by means of the CLEAN algorithm. The accuracy of this algorithm is assessed for this purpose. Finally, the gain in the Signal-to-Noise ratio is obtained and discussed.

This thesis concludes with the Chapter $\mathbf{6}$ where the general conclusions are summarized. Future research lines derived from this work are also cited at the end of this chapter. 


\subsection{Scientific Publications}

This thesis has led to 4 international journals indexed in the Journal Citation Reports, 7 international conferences, 1 national journal and 1 national conference.

Particularly, the work of Chapter $\mathbf{3}$ has been published in 3 international conferences:

- Q. Wang, D. Plettemeier, C. Andreu, C. Garcia-Pardo and N. Cardona,"Characteristics comparison of three different WCE implanted antennas in UWB low band," Proceedings of the 11th EAI International Conference on Body Area Networks (Bodynets), pp. 8-9, Turin 2016.

- C. Andreu, C. Garcia-Pardo, A. Fornes-Leal, M. Cabedo-Fabrés and N. Cardona, "UWB in-body channel performance by using a direct antenna designing procedure," 11th European Conference on Antennas and Propagation (EUCAP), pp. 278-282, Paris 2017.

- E. Miralles, C. Andreu, M. Cabedo-Fabrés, M. Ferrando-Bataller and J. F. Monserrat, "UWB on-body slotted patch antennas for in-body communications," 11th European Conference on Antennas and Propagation (EUCAP), pp. 167-171, Paris 2017.

The work of Chapter 4 has been published in 2 international journals, 3 international conferences:

- C. Andreu, S. Castelló-Palacios, C. Garcia-Pardo, A. Fornes-Leal, A. Vallés-Lluch and N. Cardona, "Spatial In-Body Channel Characterization Using an Accurate UWB Phantom," IEEE Transactions on Microwave Theory and Techniques, vol. 64, no. 11, pp. 3995-4002, Nov. 2016.

- C. Andreu, C. Garcia-Pardo, S. Castelló-Palacios, A. Vallés-Lluch and N. Cardona, "Frequency Dependence of UWB In-Body Radio Channel Characteristics," IEEE Microwave and Wireless Components Letters, vol. 28, no. 4, pp. 359-361, April 2018.

- C. Andreu, S. Castelló-Palacios, C. Garcia-Pardo, A. Fornes-Leal, A. Vallés-Lluch and N. Cardona, "Spatial In-Body to On-Body Channel Characterization Using an Accurate UWB Phantom" COST CA15104, TD(17)3025, Lisbon 2017. 
- C. Andreu, S. Castelló-Palacios, C. Garcia-Pardo, A. Fornes-Leal, A. Vallés-Lluch and N. Cardona, "UWB In-Body Radio Channel Characteristics," COST CA15104, TD(18)07014, Cartagena 2018.

- C. Andreu, S. Castelló-Palacios, C. Garcia-Pardo, A. Fornes-Leal, A. Vallés-Lluch and N. Cardona, "Spatial UWB In-Body Channel Characterization by Using Novel Phantoms at 5G Frequencies," Arco 5G workshop at IEEE International Symposium on Personal, Indoor and Mobile Radio Communications, Valencia 2016.

The work of Chapter $\mathbf{5}$ has been published in 1 international journal:

- C. Andreu, S. Castelló-Palacios, C. Garcia-Pardo and N. Cardona, "Experimental Assessment of Time Reversal for In-Body to In-Body UWB Communications," Wireless Communications and Mobile Computing (Hindawi), vol. 2018, Article ID 8927107, 12 pages, 2018.

The content of this thesis has been published in 1 international journal and 1 national journal of scientific dissemination. In addition, this content will be presented in a national conference as well:

- C. Garcia-Pardo, C. Andreu, A. Fornes-Leal, S. Castelló-Palacios, S. Perez-Simbor, M. Barbi, A. Vallés-Lluch and N. Cardona, "Ultrawideband Technology for Medical In-Body Sensor Networks: An Overview of the Human Body as a Propagation Medium, Phantoms, and Approaches for Propagation Analysis," IEEE Antennas and Propagation Magazine, vol. 60, no. 3, pp. 19-33, June 2018.

- C. Garcia-Pardo, C. Andreu, A. Fornes-Leal, S. Castelló-Palacios, S. Perez-Simbor, M. Barbi, A. Vila, M. Cabedo-Fabrés, V. Pons, M. Frasson, A. Vallés-Lluch and N. Cardona, "Wireless Communications for Medical In-Body Devices: Challenges for In-body Propagation," Waves Journal. June 2017.

- C. Garcia-Pardo, C. Andreu, A. Fornes-Leal, S. Castelló-Palacios, S. Perez-Simbor, M. Barbi, A. Vallés-Lluch and N. Cardona, "UWB Propagation for Medical In-Body Devices," Accepted for the URSI 2018 Symposium. Granada, Sept. 2018.

On the other hand, several works have arisen from this thesis: 
- M. Barbi, S. Perez-Simbor, C. Garcia-Pardo, C. Andreu and N. Cardona, "Localization for capsule endoscopy at UWB frequencies using an experimental multilayer phantom," IEEE Wireless Communications and Networking Conference Workshops (WCNCW), pp. 390-395, Barcelona 2018.

- S. Perez-Simbor, C. Andreu, C. Garcia-Pardo, M. Frasson and N. Cardona, "Path Loss Models for Implanted Devices in the Gastrointestinal Area at UWB frequencies," IEEE Transactions on Antennas and Propagation. Status: Under review.

\subsection{Academic Contributions}

This thesis has given rise to 1 final master project, which is related to the content of Chapter 2. This project has been awarded as the best final master project in the night of the valencian telecommunications (NTV) of 2017.

- Development, Design and Optimization of UWB antennas for In-body Communications (Estudio, diseño y optimización de antena UWB para comunicaciones intracorporales). Author: Enrique Miralles. Supervisors: C. Andreu and M. Cabedo-Fabrés. Grade A (10). 13/07/2016. 

Chapter 2

\section{Experimental Measurement Setup}

This chapter is devoted to explaining the different measuring equipment as well as the software tools that synchronize and control them. Besides, the phantom-based and in vivo measurement setups are detailed as well. 


\section{$2.1 \quad$ Introduction}

\subsubsection{Reproducing the Propagation through Living Tissues}

Implanting devices into human subjects in order to carry out validations of new radio technologies is not possible due to obvious ethical and physical reasons. Therefore, performing UWB in-body channel propagation measurements is challenging. To solve this issue, there are several ways to reproduce the transmission through body tissues:

- Complex computations by electromagnetic software tools. In literature, a large number of research studies based on measurement campaigns by using sophisticated and well-known electromagnetic simulation software tools such as Computer Simulation Technology (CST) or Ansys High Frequency Electromagnetic Field Simulation (HFSS) can be found [13], [41]-[43]. Here, accurate human anatomical models are embedded into the software. The shape of the human body is reproduced as well as the external and internal organs and tissues. To every human tissues, the complex permittivity value is assigned (dielectric constant and loss factor) within the operating bandwidth. The most popular models are the CAD and voxel models extracted from the U.S. National Library of Medicine under the Visible Human Project [44] (see Figure 2.1). Nonetheless, there are more human anatomical models of different ages, genres and even animal models [45], [46]. The greatest disadvantage of using anatomical models embedded in software tools is that these complex tools are not able to reproduce all the real conditions such as blood pressure, peristalsis, breathing, and so on. Such complex computations performed by using accurate anatomical models could imply the need of an expensive simulation cluster. Besides, the results obtained from simulation tools could be considered as an ideal case. This is due to the fact that other pieces of equipment involved in the communication scenario are not taken into account such as the cables, the antennas after manufacturing process, and so on.

- Experimental measurements. Using antennas, pieces of equipment and so on. This group can be split into two subgroups:

- Laboratory facilities. Chemical solutions, which emulate the electromagnetic behavior of human body tissues (i.e., complex relative permittivity), have been used in order to recreate the propagation through human tissues. These mimicking materials are known as 
"phantoms" and have been used in previous propagation studies [13], [47]. The main benefit of performing laboratory tests with phantoms is that this is a cost-effective solution and the measurements are relatively easy to perform on a daily basis. Besides, a large amount of measurements can be performed due to the high availability of the facilities. Figure 2.2 shows a phantom-based measurement setup. The main drawback is that the phantom needs to be tuned in the whole band under test as accurate as possible [39]. This may not be an inconvenient in narrow band systems since in a strait bandwidth the complex permittivity is practically constant. Nevertheless, reproducing the complex permittivity of human tissues in a large bandwidth can be an unrealizable task since these values vary at each frequency point. In fact, only a few number of phantoms are provided for wide frequency bands. An aqueous solution of sugar has been reported as UWB phantom [40], but its behavior is far from the real values reported in [12]. Other attempts have been made for wideband phantoms, but their accuracy decreases as frequency increases [48]. In particular, the reported phantoms of muscle for wide bands show a quite bad approximation above $4-5 \mathrm{GHz}$ [49], mainly regarding the conductivity part.

- In vivo. The measurements are performed using living animals such as pigs [50]-[52] (see Figure 2.3). It is often preferable to choose a porcine subject given the high similarities between pigs and human beings in terms of size, weight, dielectric features as well as the layout of internal organs and tissues. Therefore, using animal subjects could achieve a reliable solution to reproduce the transmission through the human tissues. However, animal experimentation is highly restricted in a large number of countries. Furthermore, the cost of each surgery as well as the low availability of the medical staff make difficult the use of this methodology with high periodicity. 


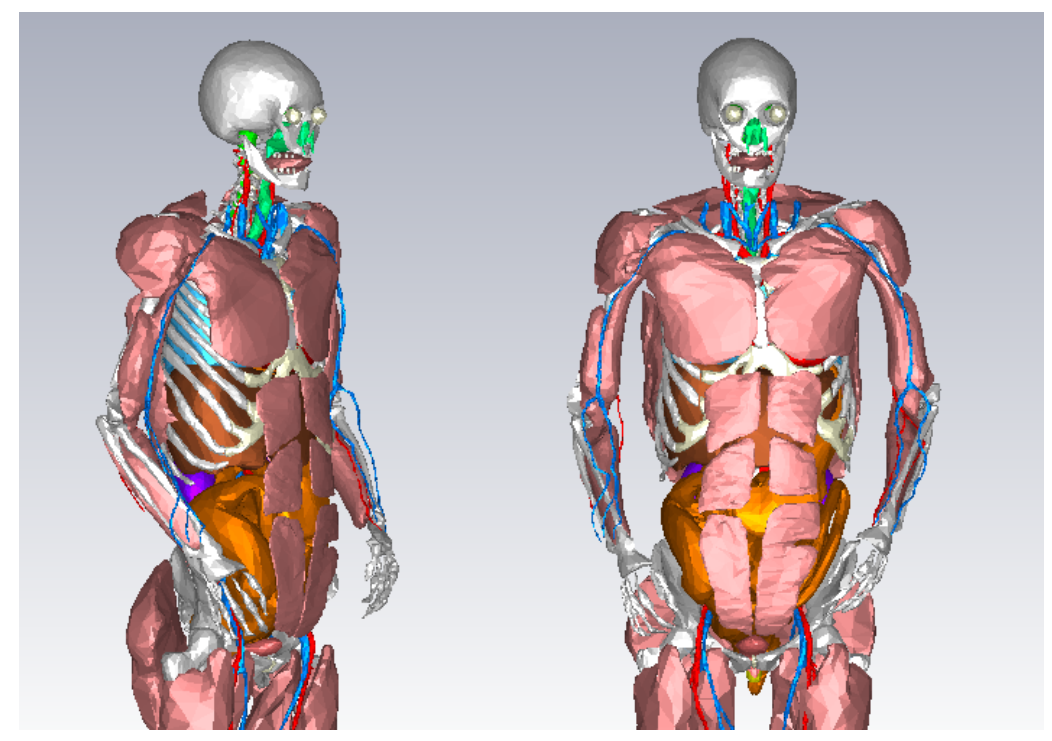

Figure 2.1: Inner tissues of the CST Female Visible Human Model "Nelly" [53].

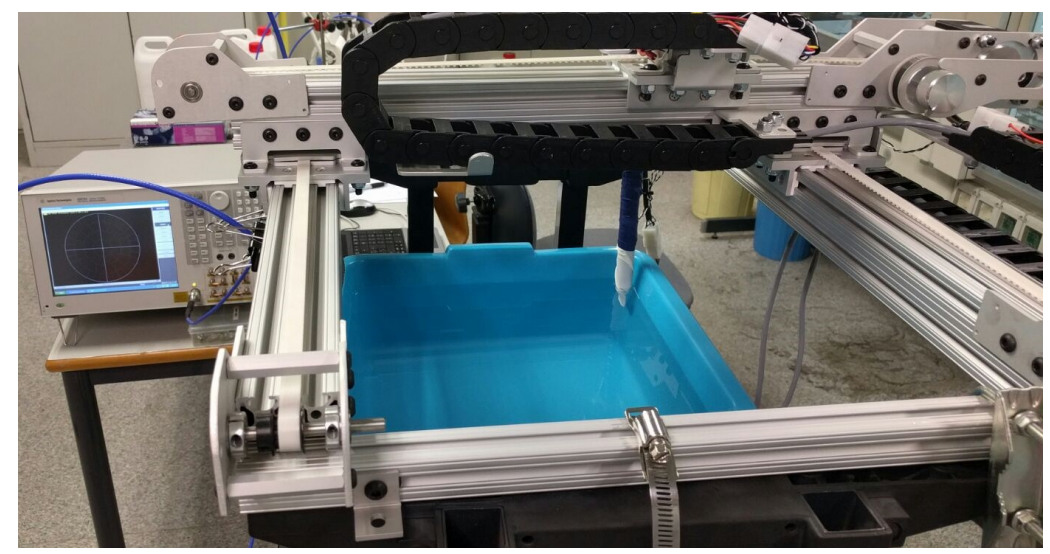

Figure 2.2: Experimental phantom-based measurement setup. 


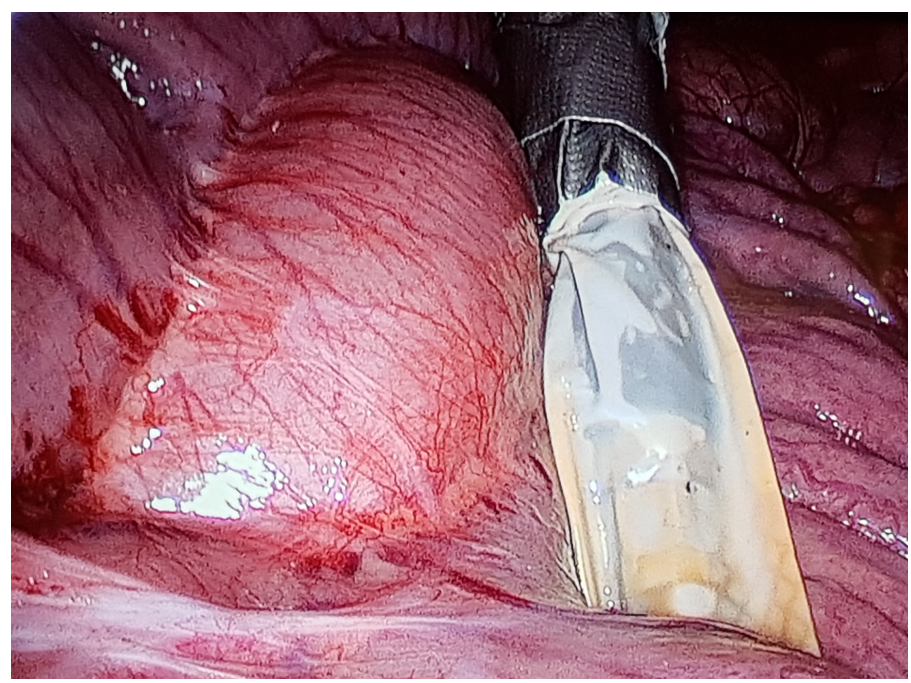

Figure 2.3: In vivo measurements. Antenna implanted in the abdominal region of a porcine subject.

\subsubsection{Frequency-Domain Channel Sounder}

Experimental measurements of the in-body propagation channel are obtained by a specific testbed. A Vector Network Analyzer (VNA) triggers a frequency sweeper to obtain the frequency response of the channel with a certain resolution (see Figure 2.4). The resolution is given by the selected bandwidth and the frequency points chosen for the sweep. The resolution in frequency impacts at the time resolution of the impulse response measurement in the time domain.

In each frequency point, the S-parameter test set sends a known signal at port 1 and monitor the received signal at port 2 . This procedure allows the VNA to determine what is known as complex frequency response of the channel $(\mathrm{H}(\mathrm{f}))$ or the forward transmission coefficient $\left(\mathrm{S}_{21}\right)$. To obtain the response in time domain, the Inverse Discrete Fourier Transform(IDFT) processing gives a band truncated version. From H(f), the channel impulse response $(\mathrm{h}(\tau))$ is obtained from the Inverse Fast Fourier Transform (IFFT) of $H(f)$ within $N$ resolution points. 


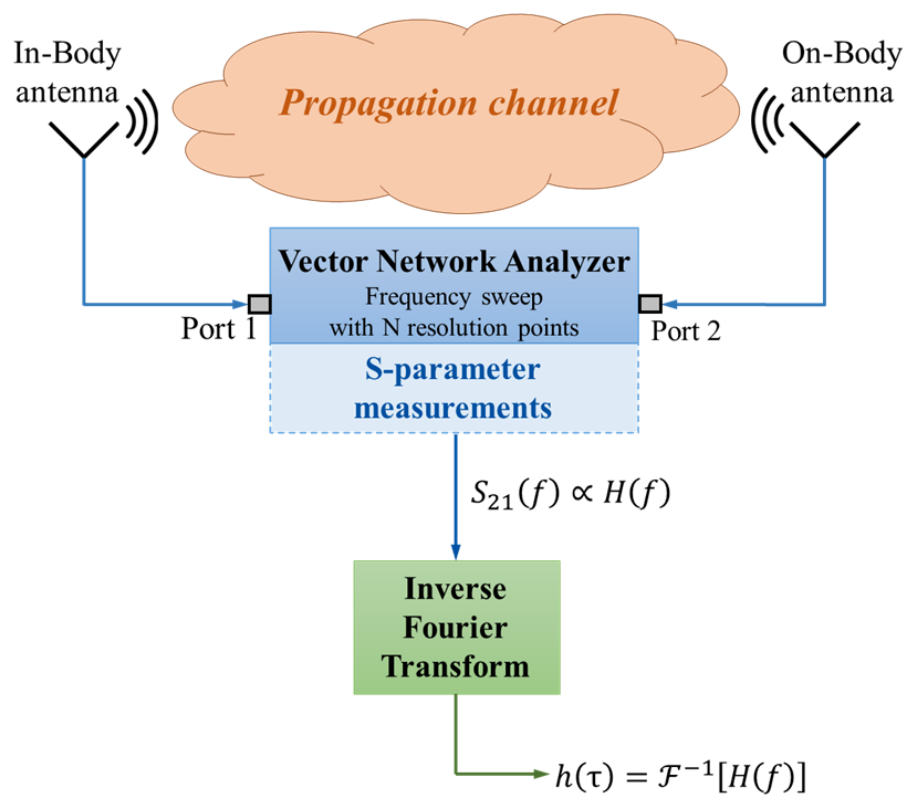

Figure 2.4: Frequency domain channel sounding [53].

Apart from measuring the $S_{21}$ parameter, other scattering parameters like the reflection coefficients are measured by the VNA. These parameters would be $\mathrm{S}_{12}, \mathrm{~S}_{11}$ and $\mathrm{S}_{22}$ in a system with 2 ports.

\subsection{Phantom-based Measurements}

In this section, in-body propagation scenarios are emulated by means of high accurate liquid phantoms which emulate the electromagnetic behavior of human body tissues within a frequency range. In this way, the UWB in-body channel can be characterized in realistic conditions.

\subsubsection{UWB Phantom}

The high restrictions in human experimentation force to find other ways to reproduce the same propagation conditions of body tissues. From an experimental point of view, it becomes necessary to employ a tissue-equivalent medium which mimics the electromagnetic behavior of the human body tissues. Phantoms, which imitate the dielectric properties (i.e., complex relative 
permittivity), have been used in order to recreate the propagation through human tissues [13].

Imitating the propagation through different tissues can be an unrealizable task. Reproducing a body multilayer model can produce inaccurate results since tissue-like layers should be separated with plastic materials or similar. Nonetheless, human body tissues located in both thoracic and abdominal region such as muscle, heart, skin or small intestine, have very similar complex permittivity values [12]. Accordingly, the use of a single-tissue phantom of the human muscle tissue is one of the most widespread approaches [42], [47]. Moreover, liquid phantoms are the most suitable in order to have free movement as well as to place implantable devices on different locations.

For our experimental measurement campaigns, an aqueous solution which imitates the dielectric properties of human muscle tissue is used. Specifically, the proposed phantom is composed of $54.98 \%$ acetonitrile and $1.07 \% \mathrm{NaCl}$. This phantom achieves the targeted conditions within UWB spectrum with high accuracy [54]. Besides, it is quite easy to prepare as follows: firstly, salt and acetonitrile are poured into a beaker in their corresponding amount. Then, the required mass is filled with water and stirred for minutes until its dissolution. Phantom has been designed to work at 24 degrees Celsius which is a common room temperature. At such temperature, the solution reproduces the dielectric values of human muscle tissue at body temperature [12]. The variation of its dielectric properties with temperature is about \pm 0.8 units per degree Celsius for the dielectric constant, and \pm 0.11 units per degree for the loss factor. The use of acetonitrile, a polar molecule that gets a dielectric constant values similar to those of muscle within a wide frequency range, is the key factor to obtain a high accurate approximation of human muscle tissue at UWB frequencies. Moreover, the addition of salt increases the loss factor so the tissue's behavior is better imitated in both parts of the complex relative permittivity (see Appendix A).

Figure 2.5 shows the complex relative permittivity of the muscle-like phantom, i.e., dielectric constant (see, Figure 2.5(a)) and loss factor (see Figure 2.5(b)), compared to the reported measured values for the real muscle tissue [12]. 


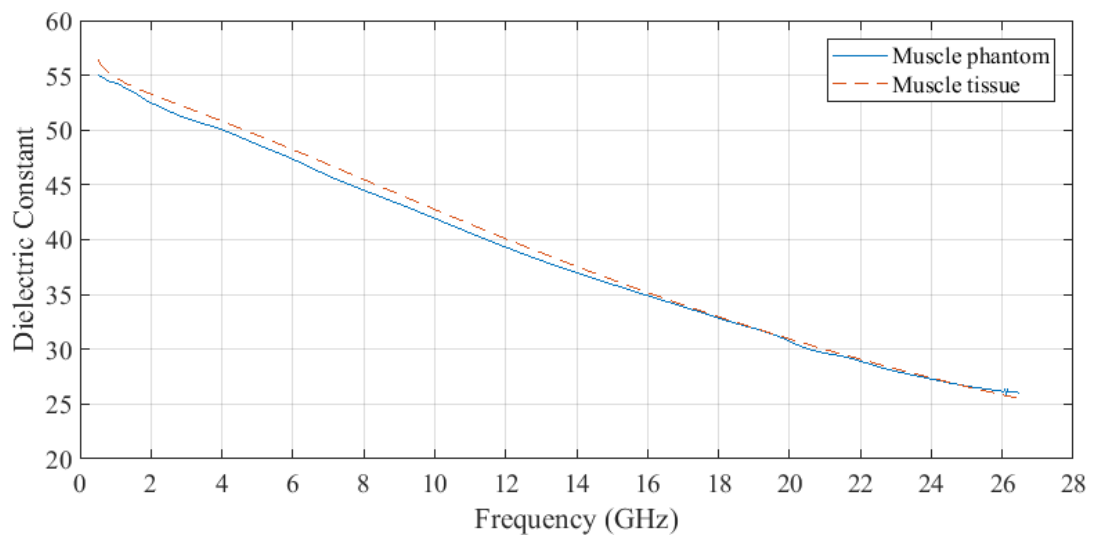

(a) Dielectric constant

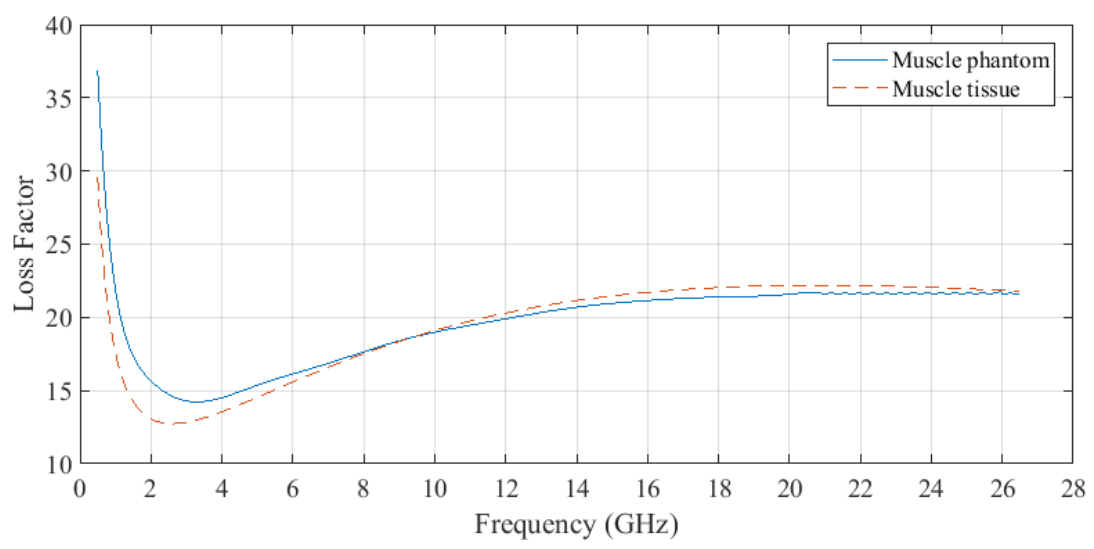

(b) Loss factor

Figure 2.5: Measured relative permittivity of the UWB phantom compared to the values of human muscle tissue reported in [12]

This phantom is the most accurate imitation of the human muscle tissue within $0.5-26.5 \mathrm{GHz}$ frequency range so far. For the phantom-based measurement setup used in this thesis, this phantom is chosen. 


\subsubsection{Measurement Setup}

For every assessed propagation scenarios, a large amount of measurements has been provided by means of a novel accurate 3D spatial experimental setup. Accordingly, a VNA, a 3-axis positioner and a tracking system are controlled and synchronized by a homemade program. In the following sections, the involved hardware and software as well as the methodology are detailed.

\subsubsection{Vector Network Analyzer}

During measurements, the four scattering parameters $\left(S_{11}, S_{22}, S_{21}, S_{12}\right)$ are obtained by means of a Keysight E5072A ENA VNA which works up to 8.5 $\mathrm{GHz}$. This piece of equipment is shown in Figure 2.6. The VNA is calibrated through a full 2-port calibration by using a calibration kit Rosenberger 03K30R in order to suppress the effect of the cables and connectors. The two antennas used in each measurement campaign are connected to port 1 and 2 of the VNA by means of two coaxial cables. Specifically, the transmitting and receiving antennas are connected to port 1 and 2, respectively.

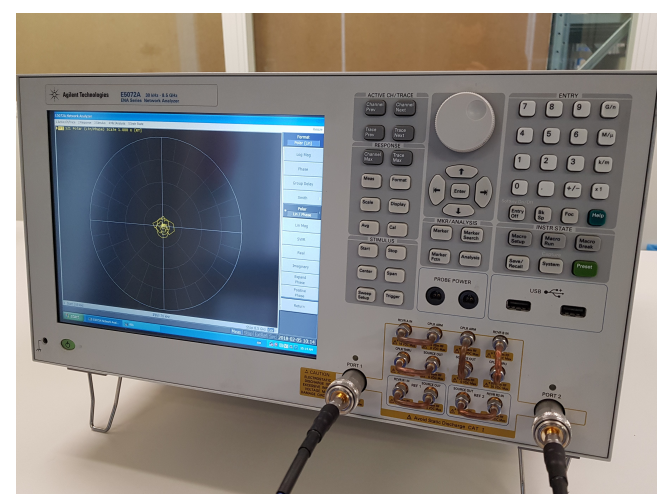

Figure 2.6: Keysight Vector Network Analyzer.

\subsubsection{Tracking System}

In order to characterize the propagation channel, the distance between transmitter and receiver must be known. For our experiments, this distance is obtained by a trackstar ${ }^{T M} 3 \mathrm{D}$ electromagnetic tracking system. This guidance system consists of a mid-range transmitter (hereafter referred to as tracking unit), two magnetic sensors and a desktop electronics unit (see Figure 2.7). 
Each sensor has a diameter of $0.9 \mathrm{~mm}$. The tracking unit generates a tracking volume and measures the absolute position XYZ of the sensors within this volume. The origin of coordinates is located at the center of the tracking unit.

The scale factor of the generated volume can be set. This is related to the size of the area that the generated volume covers. As the scale factor increases, the precision of the system decreases. This scale factor is represented by the values 36,72 and 144 in inches.

From the absolute location of the sensors, the distance between them is computed. The error of the tracking system is lower than $1.4 \mathrm{~mm}$ per sensor. The tracking unit and sensors are connected to the desktop electronics unit which integrates the power supply as well as the USB port to send the collected data to an external device such as a computer, laptop, etc.

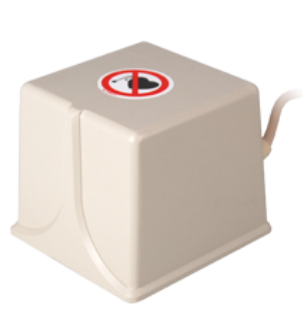

(a)

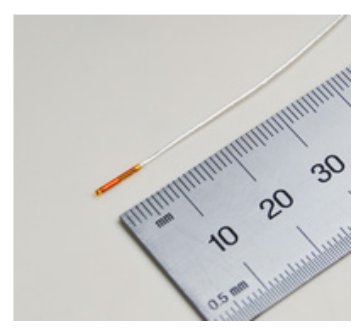

(b)

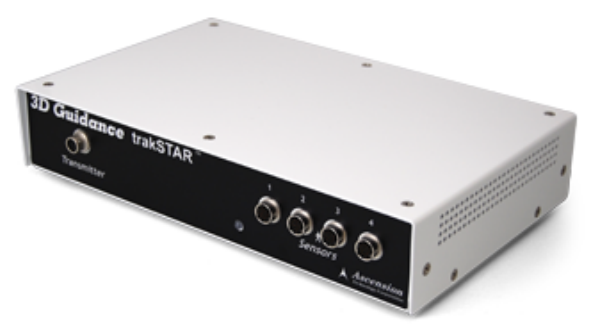

(c)

Figure 2.7: Tracking system. (a) Mid-range transmitter, (b) sensor, (c) Desktop electronics unit [55]. 


\subsubsection{3-axis Automatic Positioner}

The study of the propagation channel in a large amount of in-body locations is carried out mounting the transmitting antenna in a robotic arm (hereinafter referred to as the positioner). Three coils rotate a sheave system which moves the robotic arm across the three spacial directions. The coils are powered by two stepper motors. One step is equivalent to a movement of the robotic arm of $0.05 \mathrm{~mm}$. The error of this positioner is lower than $0.254 \mathrm{~mm}$ per $\mathrm{cm}$ in each axis.

Moving one, two or three coils at the same time, the positioner can trace a linear movement in either one, two or three axes. The origin of coordinates or the reference position of the positioner is located in one of the corners as can be observed in Figure 2.8. From this position, the robotic arm can be moved into a XYZ mesh of $30 \times 30 \times 10 \mathrm{~cm}^{3}$. The positioner can be moved to absolute locations into the XYZ mesh, e.g., from $(0,0,0)$ to $(15,12,3)$. Also, the positioner enables to perform relative movements in every axes.

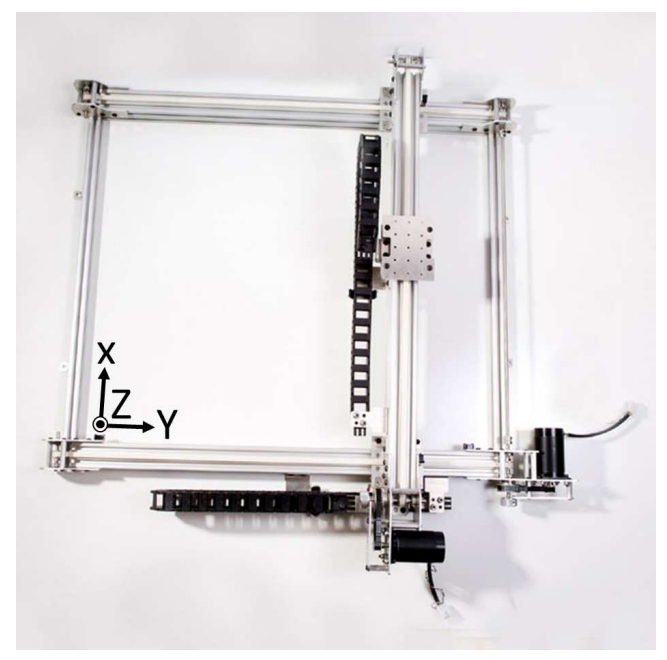

Figure 2.8: XYZ linear positioners [56]. 


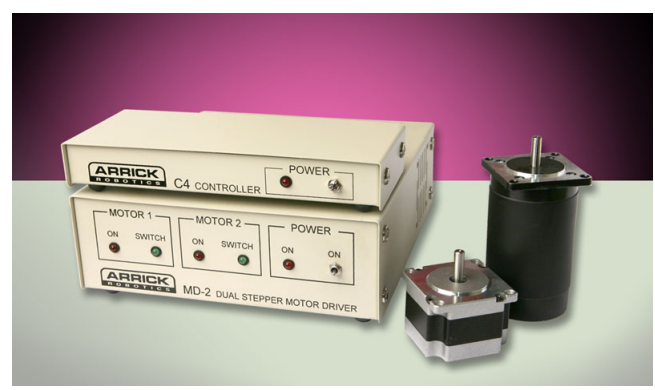

Figure 2.9: Step motors and coils [56].

\subsubsection{Measurement Setup}

In this section, the phantom-based measurement setups used in each propagation scenario are described. Two antennas which act as transmitter and receiver are used. According to the standard [8], two different scenarios are reproduced:

- In-Body to In-Body (IB2IB), where both antennas are considered to be located inside the human body ( $\mathrm{S}_{1}$ scenario in the standard).

- In-Body to On-Body (IB2OB), where the in-body transmitting antenna is considered to be located inside the body whereas the on-body receiver is placed over the human body surface $\left(\mathrm{S}_{2}\right.$ scenario in the standard).

In order to reproduce the communication for WBAN networks in a reliable way, the elements involved in the reproduction of the propagation scenarios play a crucial role. The phantom container should emulate a cross section of the human body to obtain realistic results. In the scenarios previously described, a Polypropylene (PP) container has been used. The container dimensions are $30 \times 30 \times 15 \mathrm{~cm}^{3}$ with negligible wall thickness. These dimensions have been chosen to imitate a cross section of the human body torso [41]. In fact, the considered values are widely used in simulation setups using voxel models which are designed from the dimensions of real human bodies [57]. The liquid phantom is poured into this $\mathrm{PP}$ container. Before being submerged into de UWB phantom, implantable antennas are covered with a layer of latex so that the physical contact between antennas and liquid phantom is avoided. the choice of the latex rubber is because it is widely used in healthcare environments. The antennas are carefully wrapped in order to remove the air as well 
as achieve an air-tight seal. Previously, the magnetic sensors are attached to the center of both antennas. It is worth mentioning that it has been tested that the attached sensors do not affect to the radiation parameters of the antennas at all.

During measurements, the positioner held and placed the transmitting antenna within a XYZ mesh into the liquid phantom. From an initial position, the in-body transmitter is moved by the positioner in steps of $\Delta \mathrm{x}, \Delta \mathrm{y}, \Delta \mathrm{z}$ along the three axes (relative movements) to a final position describing a rectangular grid of measurement points. The receiver is fixed either into the phantom (IB2IB, see Figure 2.10) or over the container (IB2OB, see Figure 2.11). During measurements, the positioner stopped in each measurement point until the VNA and the tracking unit had finished the data acquisition. The tracking unit is placed close enough so that the generated tracking volume completely covered the area where the measurements are performed

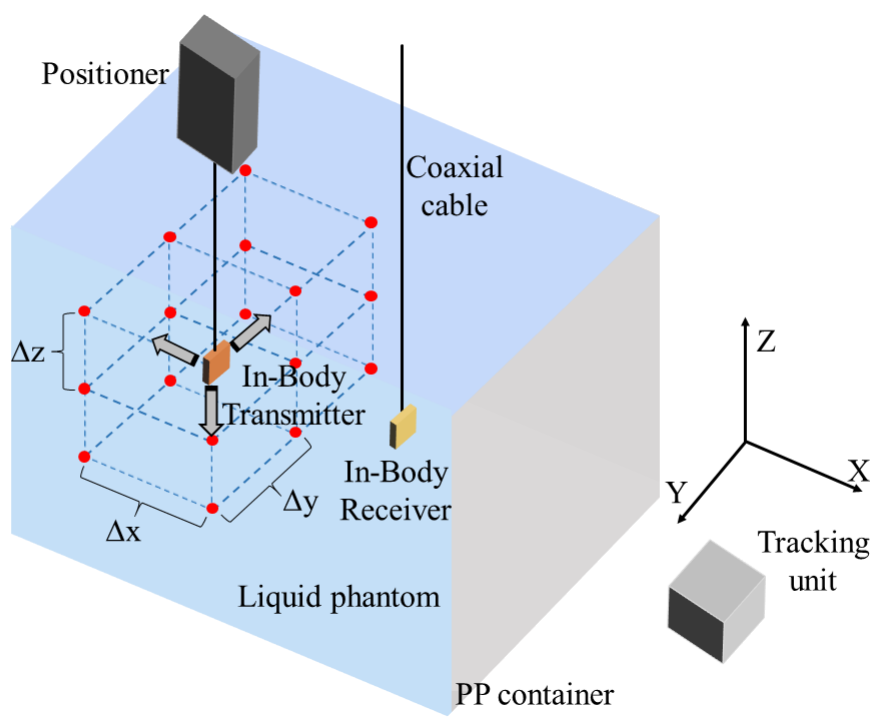

Figure 2.10: IB2IB phantom-based experimental measurement setup 


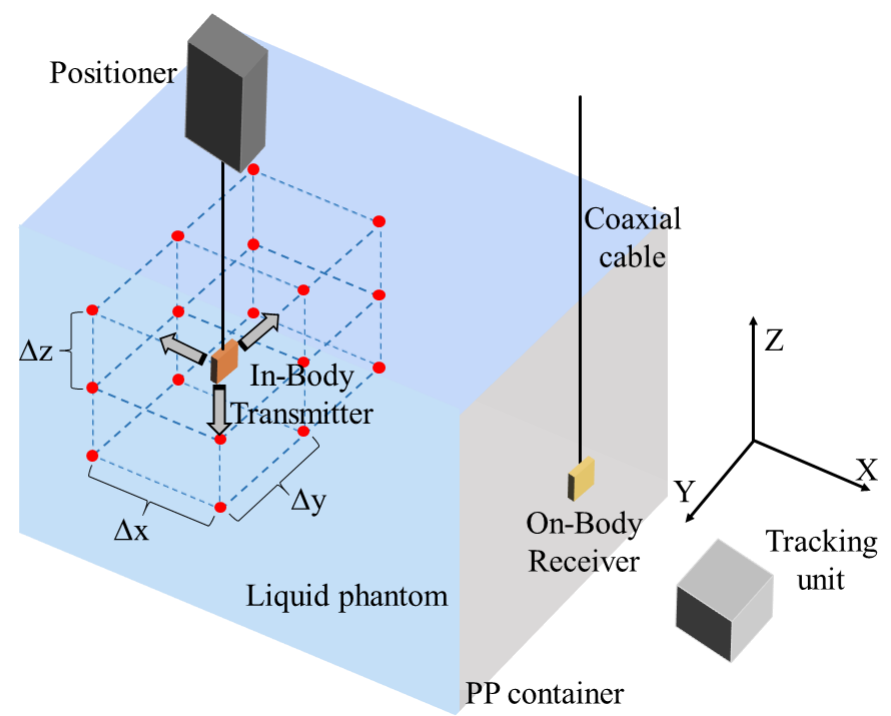

Figure 2.11: IB2OB phantom-based experimental measurement setup

\subsubsection{Custom Measurement Software}

As mentioned in previous sections, the information related to distance between antennas is provided by the tracking system; the scatter parameters are measured by the VNA; and the antenna is moved by the positioner to $(\mathrm{x}, \mathrm{y}, \mathrm{z})$ locations in a XYZ mesh. In order to work in a coordinated manner, the three pieces of equipment must be controlled and synchronized since they work independently to each other. Besides, the direct intercommunication among devices is not possible because they use different programming languages. Therefore, a software tool is needed to send and receive the commands of each device and manage them properly. For that, a custom software has been programmed to solve this task. Visual Basic (VB) has been the chosen programming language due to the fact that VB owns a large number of useful interoperability libraries.

In Figure 2.12 the main interface of the custom software is shown.

As can be observed in Figure 2.12, there are three buttons at the center of the window and two panels named as Positioner and VNA. The description of each field of the VNA panel are the following:

- Start Freq (GHz). Initial frequency value in Gigahertz $(\mathrm{GHz})$. 


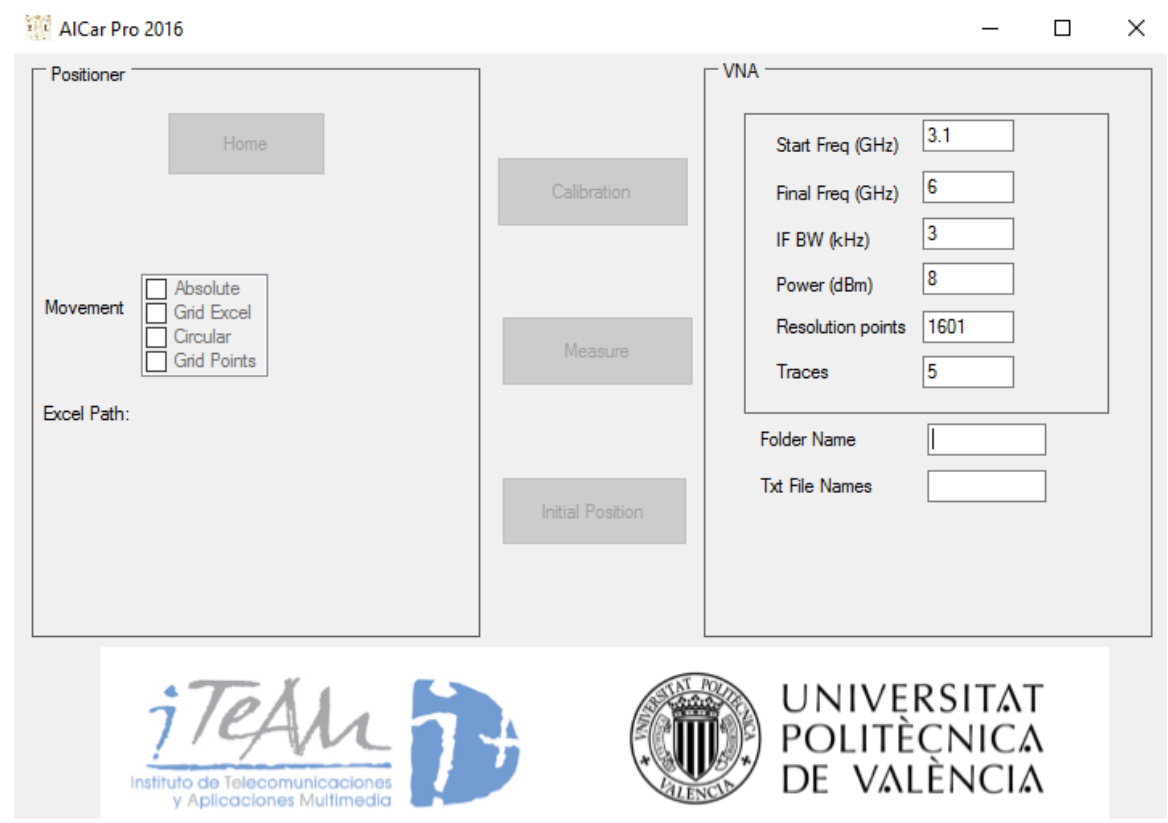

Figure 2.12: Custom program interface for phantom-based measurement campaigns.

- Final Freq (GHz). Final frequency value in Gigahertz (GHz).

- IF BW (kHz). Resolution bandwidth in kilohertz $(\mathrm{kHz})$.

- Power $(\mathbf{d B m})$. Output power from the ports of the VNA in decibelmilliwatts $(\mathrm{dBm})$.

- Traces. Number of the traces that the VNA takes of each scatter parameter in a measurement point.

- Folder Name \& Txt File Names. The software creates a folder name where the measurements will be saved. The measured values of the VNA and the tracking unit dump the collected data into this folder.

Regarding the Positioner panel, the following options can be configured:

\section{- Movement}

- Absolute. The positioner moves the robotic arm from the reference location to a position entered by the user. 
- Grid Excel. The positioner moves the robotic arm from the reference position to different locations which are indicated in a excel file. The path of the excel file is shown on the positioner panel as "Excel Path:".

- Circular. The robotic arm describes a circle centered in the middle of $\mathrm{XY}$ plane. User has to enter the radius in $\mathrm{cm}$.

- Grid Points. The positioner moves the robotic arm into a XYZ mesh describing a grid of measurement points. The step resolution (Deltax, Deltay, Deltaz) is entered by the user. The user also established the initial and final points of the grid.

Finally, the buttons located at the center of the software interface have the following functionality:

- Calibration. Open a new window to calibrate the VNA and the positioner. The VNA takes the values entered on the VNA panel (right panel in Figure 2.12) and the positioner is calibrated according to the movement selected on the Positioner panel (left panel in Figure 2.12).

- Measure. Take a trace of the forward transmission coefficient $\left(S_{21}\right)$.

- Initial Position. Change the reference location to the position where the positioner is currently located.

The software activates and deactivates its functionalities following a protocol. First of all, the devices have to be calibrated. The software has been designed to do a sequential calibration. Firstly, the VNA is calibrated through a full 2-port calibration. Then, the positioner and the tracking system are calibrated as well. Once all the devices are calibrated, the software enables the functionalities of the Positioner panel as well as the buttons Measure and Initial Position.

As can be observed in the Positioner panel, four kind of movements can be selected. Throughout this work, the Grid Points option is chosen to measure within a XYZ mesh of measurement points with the resolution entered by the user. In each point of this XYZ mesh, the positioner stops and the VNA and the tracking system measure the scattering parameters and the distance between antennas, respectively. On the one hand, the number of times that the VNA measures the scattering parameters in a measurement point is entered by the user in the field Traces. On the other hand, 500 measurements of the sensor locations in each spatial point are obtained and averaged in order to reduce the precision error of the tracking system. 


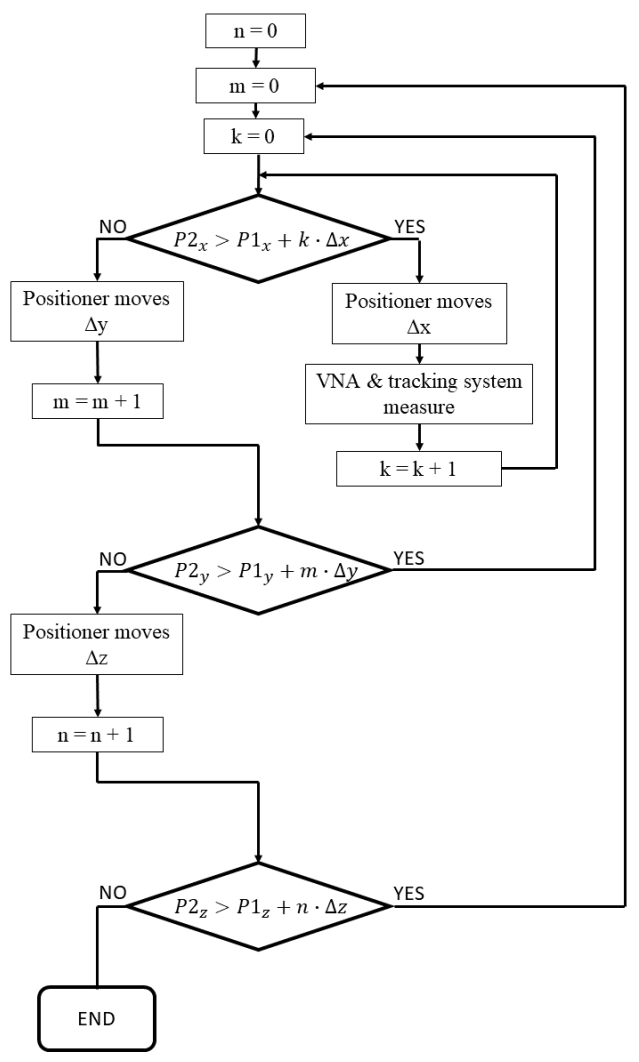

Figure 2.13: Algorithm of Grid Points movement.

The algorithm that the software follows in a measurement campaign choosing Grid points is detailed in Figure 2.13. The user has to enter a initial position $P 1\left(P 1_{x}, P 1_{y}, P 1_{z}\right)$, a final position $P 2\left(P 2_{x}, P 2_{y}, P 2_{z}\right)$ and the resolution in each axes $(\Delta \mathrm{x}, \Delta \mathrm{y}, \Delta \mathrm{z})$ in $\mathrm{cm}$. The number of points in which the positioner stops in each axis are $k_{\max }=\left\lceil\frac{P 2_{x}-P 1_{x}}{\Delta x}\right\rceil, m_{\max }=\left\lceil\frac{P 2_{y}-P 1_{y}}{\Delta y}\right\rceil$ and $n_{\max }=$ $\left\lceil\frac{P 2_{z}-P 1_{z}}{\Delta z}\right\rceil$, being $P 2_{x} \geq P 1_{x} ; P 2_{y} \geq P 1_{y}$; and $P 2_{z} \geq P 1_{z}$. Firstly, the positioner is moved $\Delta \mathrm{x}$ in $\mathrm{X}$ axis from the initial position $\left(P 1\left(P 1_{x+\Delta x}, P 1_{y}, P 1_{z}\right)\right)$. Then, the positioner stops until the VNA and the tracking system measure the scattering parameters and the distance between antennas. Once both pieces of equipment have ended the measurements, the counter $k$ is updated and this 
procedure is repeated until $P 2_{x}<P 1_{x}+k \times \Delta x$. Afterwards, the positioner goes back to the initial position in $\mathrm{X}$ axis $(k=0)$ and is moved $\Delta \mathrm{y}$ in $\mathrm{Y}$ axis. The same occurs in the $\mathrm{Z}$ axis in steps of $\Delta \mathrm{z}$. In essence, there will be a XYZ mesh of $k \times m \times n$ measurement points.

\section{$2.3 \quad$ In vivo Measurements}

Despite the low availability of surgical rooms and staff, the validation of phantom-based laboratory tests by means of measurements with living tissues is highly relevant. Living subjects is a multilayer tissue model consisting of organs, skin, bones and so on. Besides, the blood flow, the breathing and other physiological phenomena of living tissues form the structure of a living body. Hence, the differences between phantom-based and in vivo measurement results must be checked to confirm the reliability of the first ones. In this section, the in vivo measurement setup is detailed. In order to perform these measurements, a pig subject is chosen due to the high similarities between pig and human anatomy. Since the time into the surgical room is limited, only IB2OB measurements have been performed.

\subsubsection{Living Animal Subject}

The animal subject used to the in vivo measurement campaign is a female pig which weighs $80 \mathrm{~kg}$, approximately. Previously to the animal experimentation, two strict ethical committees have been approved by public organisms such as the Hospital Universitari i Politècnic La Fe (HULAFE), the Universitat Politècnica de València (UPV) and other regional authorities. The useful purpose of the measurements and the whole detailed medical procedure had to be demonstrated in the aforementioned committees.

\subsubsection{Measurement Setup}

Likewise the IB2OB scenario described in section Section 2.2.2.4, the in-body antenna acted as a transmitter, whereas the on-body one acted as a receiver. In this case, the on-body receiver is in direct contact with the animal skin. The in-body antenna is covered by latex rubber in order to avoid the damage of the antenna due to the internal fluids. Previously, a sensor of the tracking system is attached to the center of the antenna. Furthermore, other sensor is attached to the on-body receiver to compute the distance between antenna centres. As in the phantom-based setup, the antennas are connected to the 
ports 1 and 2 of the VNA by means of two coaxial cables. The attached sensors are connected to the desktop electronics unit by two flexible cables provided by the manufacturer. More details about the VNA and the tracking system can be found in Section 2.2.2.1 and Section 2.2.2.2, respectively.

Firstly, the living porcine subject is anesthetized. Afterwards, the in-body antenna is placed in several locations of the abdominal region (see Figure 2.14). Specifically, the antenna is placed among the guts of the small intestine by means of a laparoscopy (see Figure 2.15). The abdominal region of the pig subject is filled with carbon dioxide $\left(\mathrm{CO}_{2}\right)$ up to 14 millimeters of mercury $(\mathrm{mmHg})$ of pressure. Then, the in-body antenna is placed in the desired location using tweezers. The correct placement of the in-body antenna is controlled by a internal camera. Once the antenna is implanted in the sought position, the belly is emptied of $\mathrm{CO}_{2}$.

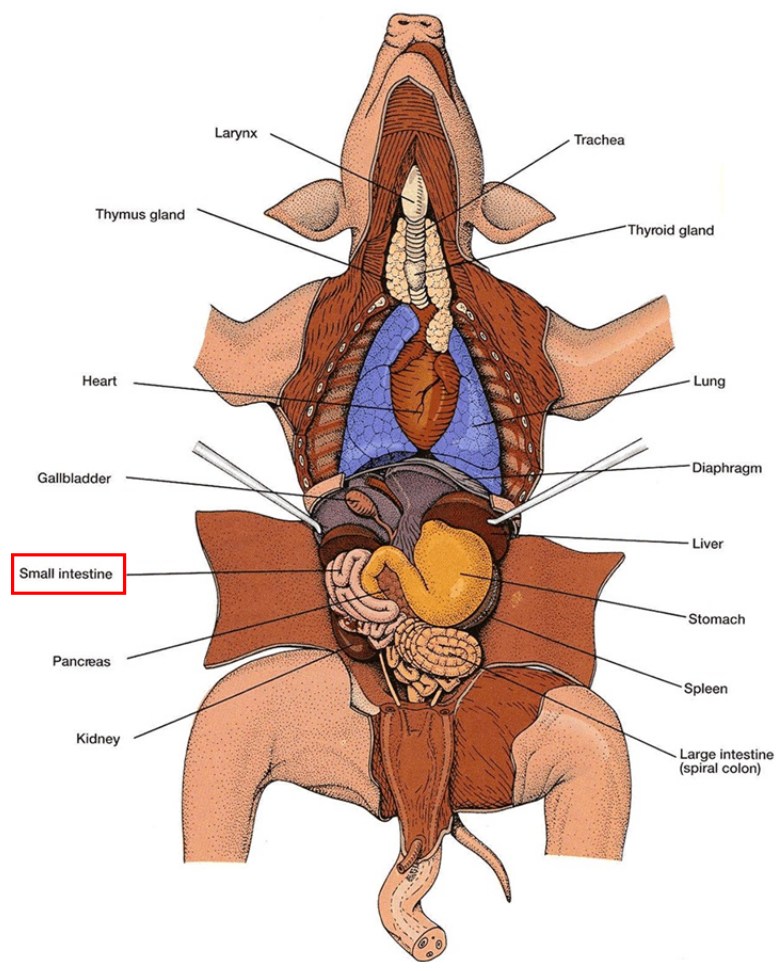

Figure 2.14: Pig anatomy [58]. 


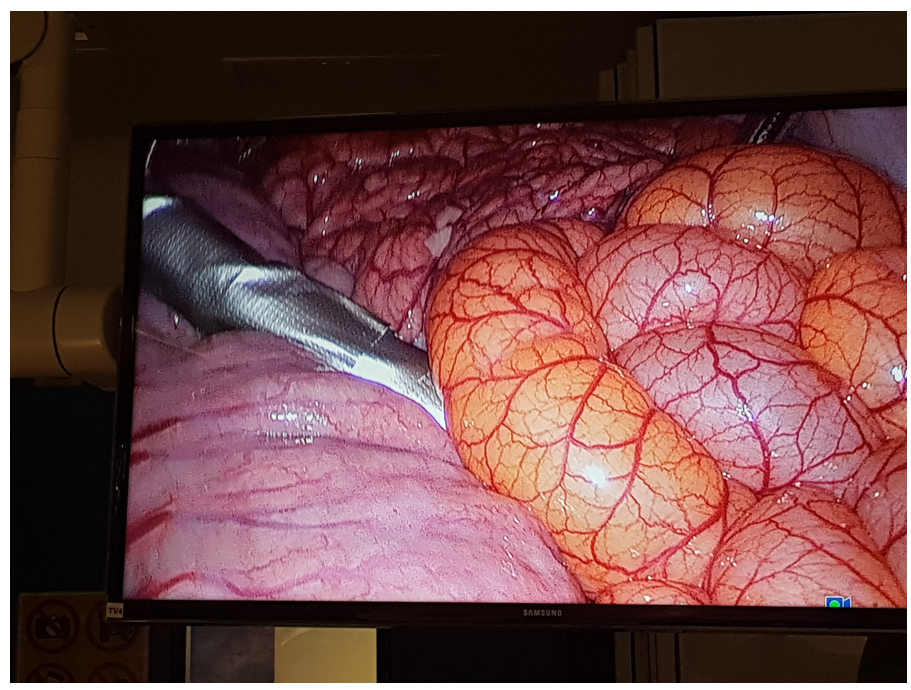

Figure 2.15: In-body antenna placed among the guts of the small intestine. Picture taken by the laparoscopic camera.

Whereas the in-body antenna is fixed in a location inside the animal subject, the on-body antenna is placed in several positions over the pig's belly. The on-body receiver is placed in several ventral locations (positions numbered from 1 to 13 in Figure 2.16). These positions are separated multiples of $\lambda$, being $\lambda$ the wavelength corresponding to the central frequency of the frequency band under test considering the propagation speed in the muscle tissue. In each of these on-body positions, the VNA and the tracking unit measure the scattering parameters and the position of the antennas, respectively. It should be mentioned that the on-body antenna is covered by a isolator layer made by a ferrite material to avoid the creeping waves and other external contributions (see Figure 2.17).

\subsubsection{Measurement Software}

In order to perform the in vivo measurements, the VNA and the tracking system have to be used to collect the values of the scattering parameters and the location of the transmitter and the receiver, respectively. According to that, a modified version of the software described in Section 2.2.2.5 has been programmed for this purpose. For this particular case, the commands to control the VNA and the tracking system are only included. Figure 2.18 shows the 


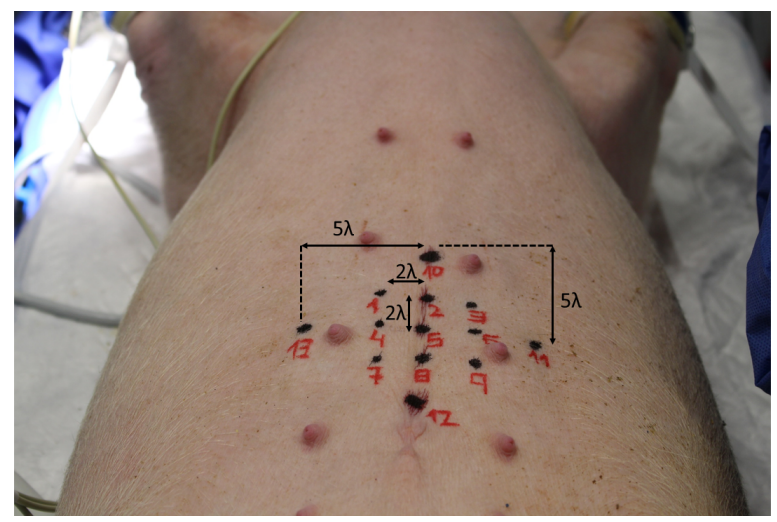

Figure 2.16: Locations of the on-body receiver over the pig's belly.

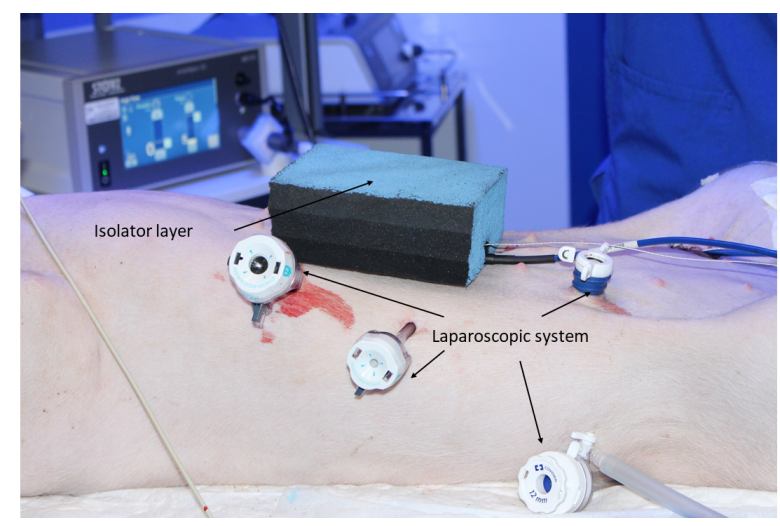

Figure 2.17: On-body receiver placed on the belly and covered by the isolator layer.

interface of the custom program for this purpose. As can be seen in Figure 2.18, the new software has three main panels. The Calibration panel located at the top of the window calibrates both the VNA and the tracking system by means of the button named as Calibrate. The VNA is pre-configured with a particular configuration (frequency, power, etc.) in order to avoid unnecessary waste of time since the time inside the surgical room is limited. The VNA is calibrated through a full 2-port calibration with the following parameters:

- Output power $=8 \mathrm{dBm}$

- Resolution bandwidth $=3 \mathrm{kHz}$ 


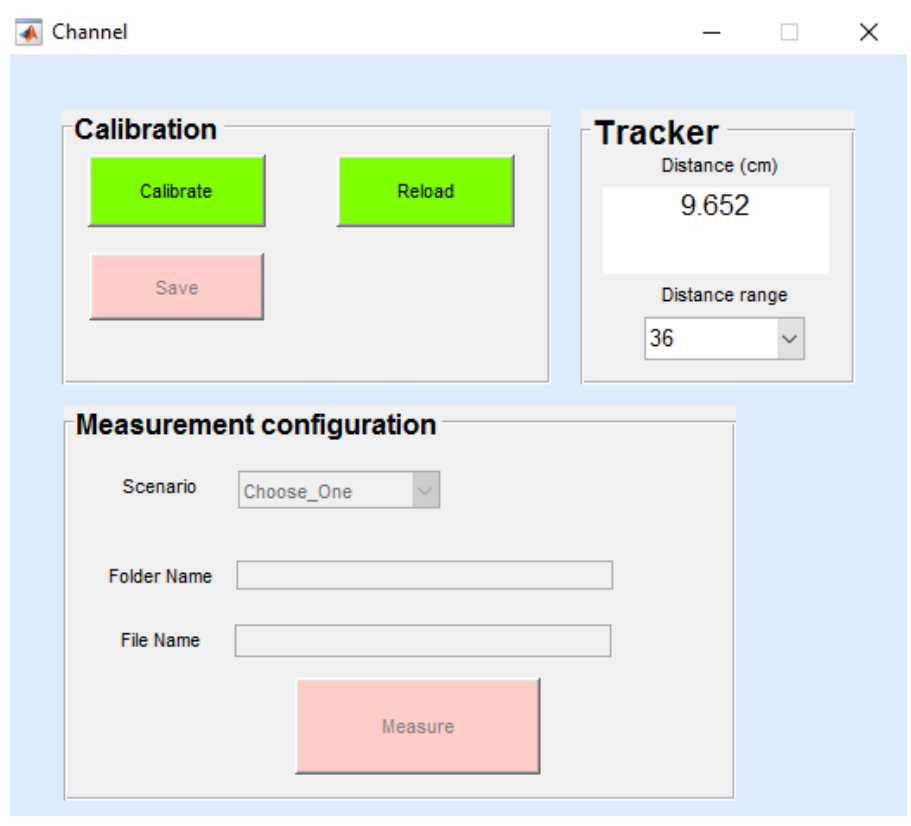

Figure 2.18: Custom software interface for in vivo measurement campaigns.

- Initial frequency $=3.1 \mathrm{GHz}$

- Final frequency $=5.1 \mathrm{GHz}$

- Resolution points $=1601$

- Traces $=5$

The Save and Reload buttons save the current calibration files and load the last saved calibration in both devices, respectively. The Tracker panel shows in real-time the distance between the sensors of the tracking system. The refresh time of the display is 0.5 seconds. Knowing the distance between sensors in real-time is relevant to know how deep the inner antenna is. The size of the generated volume by the tracking unit can be selected in this panel as well.

The Measurement Configuration panel includes three fields and a button:

- Scenario. A folder with the scenario name is generated. 
- Folder Name. A folder with the name detailed in this field is generated into the scenario folder.

- File Name. The name of each file which includes the measurements from the VNA and the tracking system.

- Measure. By clicking on this button, the VNA and the tracking system measure. Then, a file is filled with the collected measurements.

As in section Section 2.2.2.5, the tracking system takes 500 measurements of the current position of the sensors attached on the antennas. Each measurement of the tracking system and VNA takes 20 seconds, approximately. The procedure that the software follows to calibrate, name the files and measure is depicted in Figure 2.19. Firstly, the VNA and the tracking system are calibrated. Then, when the Measure button is clicked, the VNA measures the scattering parameters and the tracking system takes the position of the attached sensors. The obtained values are saved in the file and folders named by the user. During the whole measurement campaign, the distance between antennas is shown in the Tracker panel depicted in Figure 2.18.
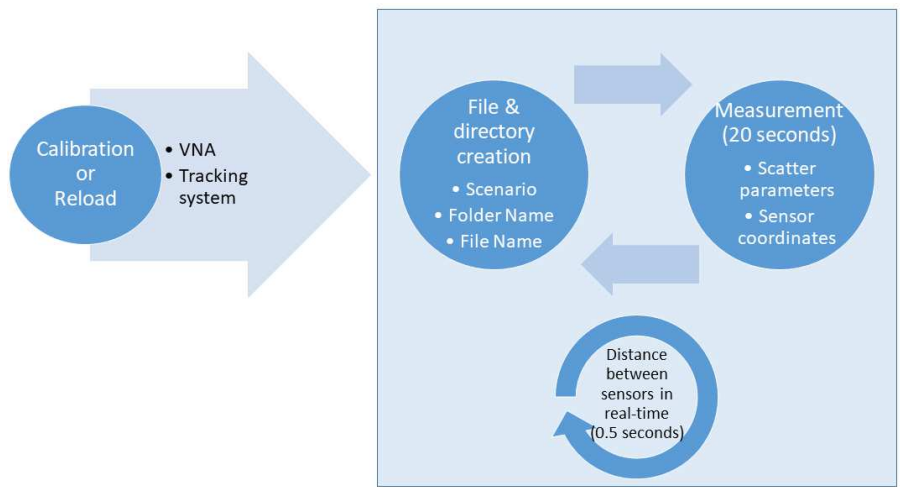

Figure 2.19: Algorithm of the software for in vivo measurements. 



\section{Chapter 3}

\section{UWB Biomedical Antennas}

In order to assess the UWB channel performance in a reliable way, the antennas involved in the communications with implants play a key role. In this chapter, two procedures to miniaturized and optimized UWB antennas for inbody communications are assessed and discussed. On the one hand, a new antenna model is designed by means of the most extended procedure of antenna miniaturization. This part of the thesis are the result of a short-term stay in the Technische Universität of Dresden. On the other hand, a new approach to design implantable and on-body antennas is tested. 


\subsection{Introduction}

In order to achieve a realistic experimental characterization of the UWB inbody channel, the antennas involved in the communication play a crucial role. The radio channel characteristics is strongly influenced by the antenna behavior in this propagation medium [43]. In-body, on-body and off-body antennas must comply specific requirements which depend on the medical application.

\subsubsection{Antenna Fundamentals}

\subsubsection{Antenna Parameters in a Lossy Medium}

The impedance of an antenna is defined as the ratio between the electrical tension and current at its input terminals [59]. Normally, this is a complex impedance. The real part is named as the antenna resistance, whereas the imaginary one is known as reactance. Figure 3.1 depicts the equivalent circuit of an antenna structure. The antenna is fed by a transmission line with characteristic impedance of $Z_{0}$. The antenna impedance is expressed as follows:

$$
Z_{A}=R_{L}+R_{r}+j X_{A},
$$

where $R_{L}$ and $R_{r}$ are the loss and radiation resistances, respectively, and $X_{A}$ is the antenna reactance.

Considering the expressions for an antenna working at free space, in the case of a lossy medium, the radiation resistance can be expressed from the radiated power in far field with a input current as [60]:

$$
R_{r}=\frac{P_{r}}{I^{2}}=\frac{\int P(\theta, \varphi) r^{2} \sin \theta d \theta d \varphi}{I^{2}},
$$

being $P(\theta, \varphi)$ the density power in a lossy medium expressed in spherical coordinates. The module of $P(\theta, \varphi)$ is defined as:

$$
P=\Re\left[\frac{|\vec{E}|^{2}}{Z_{m}} \cdot e^{-2 \alpha r}\right],
$$

where $e^{-2 \alpha r}$ is a decay factor to compensate the attenuation caused by the lossy medium ( $\alpha$ would be negative in a lossy medium); $\mathrm{Z}_{m}$ is the wave impedance in the lossy medium.

Also, the radiation resistance and E-field are different compared to that in free space because of the changes in the current distribution. This is by the fact that the presence of the lossy medium. 


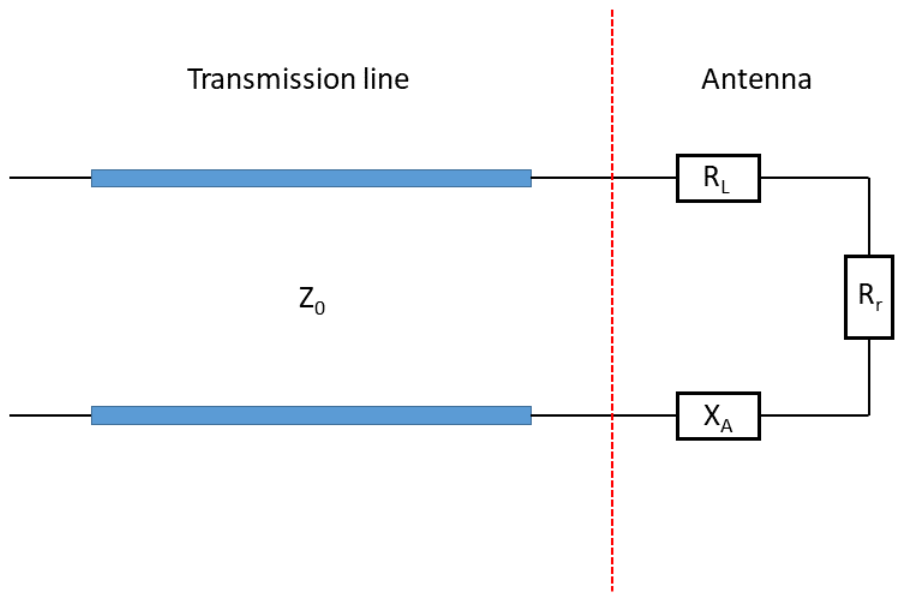

Figure 3.1: Scheme of antenna structure [61].

The antenna efficiency, $\eta$, and the reflection coefficient, $\rho$, are determined by:

$$
\eta=\frac{R_{r}}{R_{r}+R_{L}},
$$

and the reflection coefficient, $\rho$, is determined by:

$$
\rho=\frac{Z_{A}-Z_{0}}{Z_{A}+Z_{0}},
$$

being $Z_{A}$ the antenna impedance.

The impedance of the transmission line should be matched with that of the antenna in order to reduce $\rho$ as much as possible. Ideally, $\mathrm{Z}_{0}$ is the complex conjugate of $Z_{A}$. It is worth recalling that the reflection coefficient is the magnitude of the reflected power at the port connected to the antenna, i.e., $\mathrm{S}_{M N} \forall M=N$, being $M$ and $N$ less or equal to the number of ports. A really good antenna matching is achieved when the reflection coefficient is below -10 $\mathrm{dB}$ within the desired bandwidth [59]. 


\subsubsection{Radiation Pattern in Near and Far Field}

The radiation pattern is a graphic representation of the radiation properties of the antenna as a function of the space coordinates [60]. In spherical coordinates, the radiation pattern as a function of the E-field can be expressed as:

$$
C(r, \theta, \varphi)=\left.\frac{\vec{E}(r, \theta, \varphi)}{|\vec{E}(r, \theta, \varphi)|_{\text {max }}}\right|_{r=\text { constant }}
$$

Also, it can be expressed as a function of the radiated power as:

$$
T(r, \theta, \varphi)=\left.\frac{\vec{P}(r, \theta, \varphi)}{|\vec{P}(r, \theta, \varphi)|_{\text {max }}}\right|_{r=\text { constant }}
$$

Therefore, $T(r, \theta, \varphi)=C^{2}(r, \theta, \varphi)$.

Depending on the distance to the antenna, $r$, the electromagnetic radiated field can be simplified in three different region. These field regions can be determined by the maximum dimension of the antenna, $D$, and the wavelength, $\lambda$. It is worth mentioning that these conditions are valid for $D>>\lambda$.

- Reactive near field or Rayleigh region. Region influenced by the charges and current of the antenna as well as the reactive radiated power.

$$
r<\frac{\lambda}{2 \pi}
$$

- Radiating near field or Fresnel region. As the the distance to the antenna increases, the radiated fields begin to appear. However, they still depend on distance.

$$
\frac{\lambda}{2 \pi}<r<\frac{2 D^{2}}{\lambda}
$$

- Far field or Fraunhofer region. Electric and magnetic fields are not influenced by the charges and currents of the antenna in this field region. From this distance, the radiated power can be considered as a plane wave.

$$
r>\frac{2 D^{2}}{\lambda}
$$




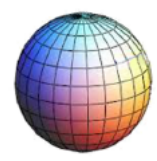

Isotropic

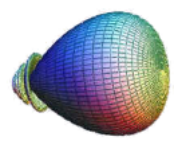

Directive

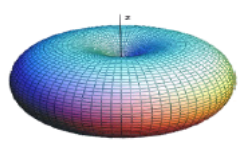

Omnidirectional

Figure 3.2: Types of radiation pattern [61].

On the other hand, three radiation patterns can be distinguished. These are the isotropic, directive and omnidirectional (see Figure 3.2). An isotropic antenna is whose radiated power is uniformly sent in all the space directions. It represents a perfect spherical shape. Actually, it is not possible to get an isotropic antenna. However, this radiation pattern is useful to compare these characteristics with other kinds of antennas. A directive antenna has a maximum of the radiated power in some direction in space. Finally, an omnidirectional antenna equally radiates in all directions of a plane.

\subsubsection{Directivity and Gain}

\section{Directivity}

The directivity is the relation between the radiated power density in a direction and the power density which an isotropic antenna would radiate at equal radiated power. The directivity can be expressed by:

$$
D(\theta, \varphi)=\frac{P(\theta, \varphi)}{\frac{P_{r}}{4 \pi r^{2}}}
$$

The maximum directivity is obtained from the radiation pattern of the antenna as:

$$
D_{\max }=\frac{P_{\max }}{\frac{\iint P(\theta, \varphi) r^{2} \sin \theta d \theta d \varphi}{4 \pi r^{2}}}
$$


Gain

The antenna gain is defined as the relation between the radiated power density and the power density which an isotropic antenna would radiate at equal distances and power supplied to the antenna $\left(P_{s}\right)$.

$$
G(\theta, \varphi)=\frac{P(\theta, \varphi)}{\frac{P_{s}}{4 \pi r^{2}}} .
$$

The antenna directivity is related to the gain. The relation between the power supplied to the antenna and the radiated power is known as ohmic losses or antenna efficiency:

$$
\eta_{\Omega}=\frac{P_{r}}{P_{s}}
$$

Hence, the relation between gain and directivity can be expressed as:

$$
G(\theta, \varphi)=\eta_{\Omega} D(\theta, \varphi) .
$$

\subsubsection{Overview of UWB antennas for Biomedical Applications}

\subsubsection{UWB Implanted Antennas}

In order to achieve a realistic experimental characterization of the UWB in-body channel, miniaturized antennas play a crucial role. The overall dimensions of implantable antennas should be suitable to be implanted inside the human body. Furthermore, achieving high data rates occupying the whole UWB bandwidth is one of the most relevant challenges of UWB antennas. Besides, in-body antennas should have an omnidirectional radiation pattern at all frequencies to communicate with an on-body sensor array, an external reception system or other implanted devices placed on different parts of the human body. For example, the capsule endoscope moves uncontrollably inside the gastrointestinal tract. By using a omnidirectional radiation pattern, the probability to losing the communication is lower than that of an antenna with a directive pattern.

As mentioned, the size and shape of the in-body antenna is constrained to the medical application in which it will be used. For instance, in [62], a UWB loop tiny antenna to be implanted inside the brain is presented. Research works of antenna designers are focused on designing efficient antenna candidates for 


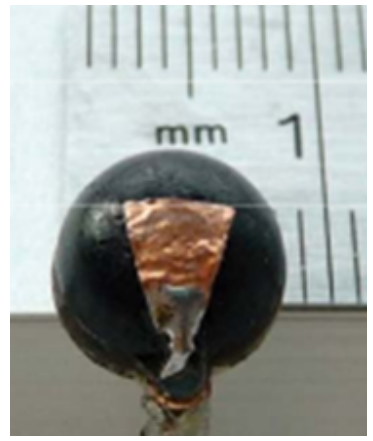

(a)

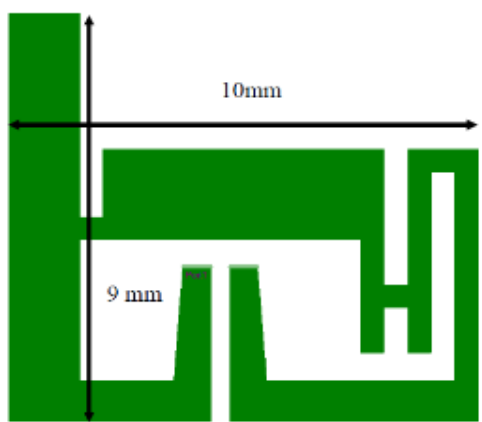

(b)

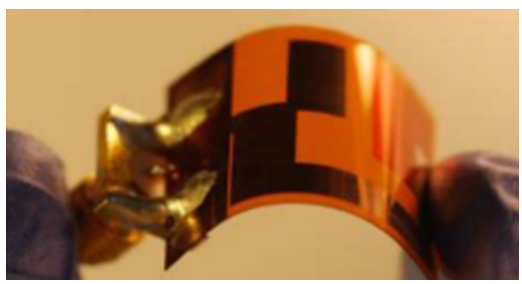

(c)

Figure 3.3: UWB implantable antennas. (a) For capsule endoscope [33]. (b) Loop antenna to be implanted into the brain [62]. (c) Flexible implantable antenna [63].

future UWB capsule endoscopies. In [33], authors bet on using a dielectric resonator antenna to be embedded in the dome of the capsule. In [64], an helical and a loop antennas are conformed over the dome, whereas authors in [63] use a flexible shaped antenna attached to the body of the capsule.

Even though UWB is a potential candidate for future in-body communications, the literature is lack of antenna candidates to work within this band efficiently. So many technologies and novel innovative design procedures must be undertaken to perform reliable validation tests within this band. 


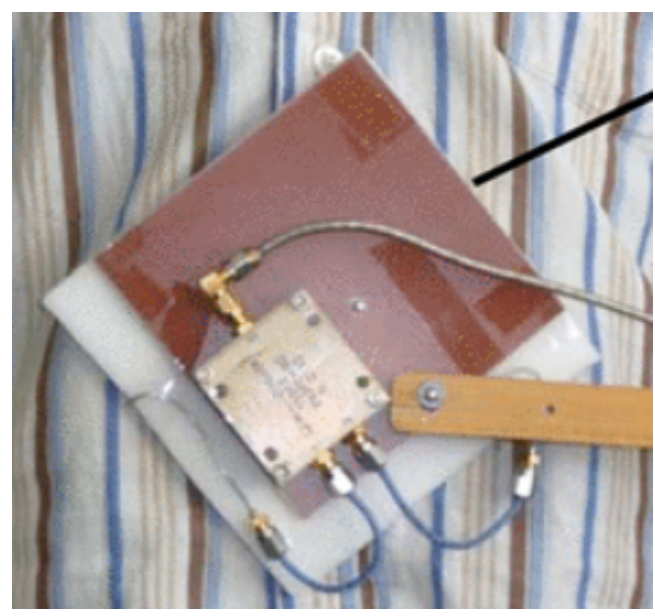

Figure 3.4: Manufactured UWB On-Body antenna model [66]

\subsubsection{UWB On-Body Antennas}

As mentioned above, according to the locations of transmitters and receivers, different communication scenarios can be considered. Regarding the IB2OB scenario, $\mathrm{S}_{2}$ in the standard, the implanted antenna is located inside the human body whereas the on-body antenna is placed over the human body surface. With regard to the on-body antenna, a compact structure to be embedded in different structures is needed. For example, on-body receivers are usually located in the patient's waist in a typical scenario to communicate with a capsule endoscope [65]. Patients do not remain in hospital and continues his daily life. So, the external system must not be too bulky.

On the other hand, concerning the antenna parameters, the antenna matching should be ensured within the whole frequency band of interest. Moreover, the radio waves within this frequency band should have fair penetration through the human tissues in order to establish a proper link with an implanted antenna. For these antennas, a directive radiation pattern is preferable instead of a omnidirectional one [66]. This is due to the fact that on-body antennas communicate with a implanted device in one direction. There are several ways to focus the power and thus reduce the backward radiation. One of them could be the inclusion of a reflecting plane [60], or even the use of an optimal sensor array [67]. 
In literature, a high number of UWB on-body antenna designs for on-body to on-body (OB2OB) and on-body to off-body (OB2OFF) communications can be found [68]-[70]. However, the literature is lack of UWB on-body antennas for in-body communications. Many more efficient antenna models for UWB such as horn, patch, slot and so on, should be designed and tested as candidates to work in this specific application.

\subsection{UWB Implantable Antennas}

In order to perform reliable UWB in-body measurements, antennas play a crucial role. In in-body communications, the channel characteristics are highly influenced by the antenna performance compared to that in free space. The harsh propagation environment could change the radiation parameters and decrease the efficiency of the antennas dramatically due to the frequencydependence of body tissues [12].

Implantable antennas have to comply specific requirements in terms of size, shape and radiation parameters such as the radiation pattern, antenna matching, and so on. To profit from UWB systems, new in-body antenna designs should achieve a bandwidth as large as possible. In this section, two design procedures for UWB implantable antennas are assessed and discussed.

\subsubsection{Typical Procedure for In-Body Antenna Models}

\subsubsection{Overview}

In-body antenna design is challenging. Implantable antenna models have particular peculiarities which have been taken into account at the design stage, unlike antenna models designed to work in free space. The propagation medium is not the air. In this context, it is essential to consider the dielectric properties of human body tissues since they can affect the antenna characteristics. The most extended procedure to design implantable antennas takes the free space validation of the antenna as a starting point. In literature, related research works take the average value of the muscle tissue at UWB frequencies in order to design a feasible UWB implantable antenna [38]. This is by the fact that the average value of the dielectric constant of the human body torso at UWB frequencies is nearly 0.6 times that of the human muscle tissue [39], [71].

As mentioned above, the propagation medium is not the free space. In fact, the effective wavelength of the antenna is shorter in comparison with the 
wavelength in free space. The relationship between the wavelength for body tissues and for free space is:

$$
\lambda_{e f f}=\frac{\lambda_{0}}{\operatorname{Re}\left[\frac{\epsilon_{r}}{\epsilon_{0}}\right]},
$$

where $\lambda_{\text {eff }}$ is the effective wavelength, $\lambda_{0}$ is the wavelength in free space, $\epsilon_{0}$ is the permittivity of vacuum and $\epsilon_{r}$ is the relative permittivity of the human body tissue.

The typical procedure to optimize and miniaturize in-body antennas profits from this shorter wavelength. Mostly, implantable antenna models are designed to work in free space in a first stage [33]. Thus, the in-body antenna is designed to work at higher frequencies since, when it is implanted into the human tissues, a shorter wavelength is expected [72]. Therefore, the resonance frequency should shift down as the antenna is implanted. A Shortening Factor (SF) of the wavelength can be considered. This SF is obtained from the relationship between the wavelength in free space and the wavelength in the human tissue as:

$$
S F=\frac{\lambda_{0}}{\lambda_{e f f}} .
$$

When the bandwidth is large, the shortening factor should be averaged since the dielectric properties of human vary with frequency [12]. At UWB frequencies, from $3.1-10.6 \mathrm{GHz}$, this $\mathrm{SF}$ is around 7.2 considering the complex permittivity values of human muscle tissue. Hence, for antenna designs at UWB frequencies, the resonance frequency of the candidate antenna should be located between 20 and $25 \mathrm{GHz}$ on free space, approximately.

\subsubsection{UWB In-Body Antenna Candidate}

The antenna model is influenced by the application. Regarding implantable or ingestible transmitters, they should comply to strict features in terms of size or shape. Hence, the most suitable antenna model for the final purposes should be carefully studied.

WCE is a widely used wireless device which takes images from the inside of the gastrointestinal tract [4]. The antenna for the WCE should comply with the constrains in terms of dimensions in order to be embedded inside the capsule. Besides, an UWB antenna for WCE should be matched within the frequency range of interest. According to the commercial capsule dimensions, the design specifications for a UWB antenna for WCE should be the following: 


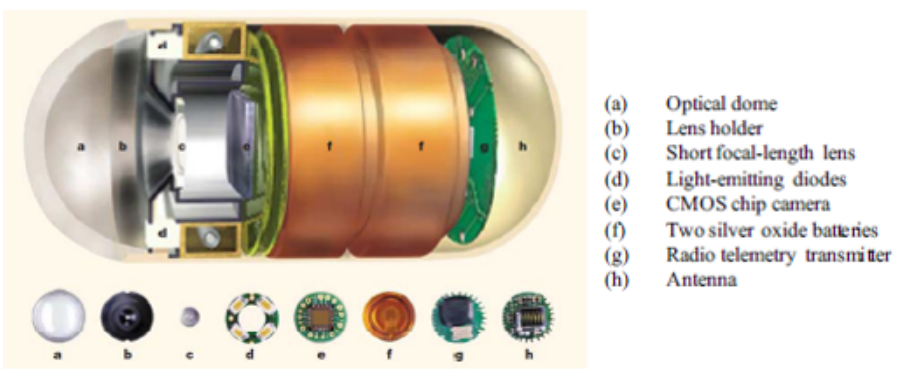

Figure 3.5: Wireless capsule endoscopy structure [33].

- Operating environment. Tissues of abdominal region. Communicating with either body surface or external antenna.

- Fitting inside capsule volume. The size of WCE is $11 \mathrm{~mm}$ in diameter and $26 \mathrm{~mm}$ in length. As can be observed in Figure 3.5, the antenna should take up one-sixth of the overall size of the capsule, approximately.

- Antenna maching within UWB frequencies. The antenna matching should be within $3.1-10.6 \mathrm{GHz}$. It is preferable that the antenna matching was at the lower part of the UWB spectrum since the losses in this region are lower [12].

- Impedance bandwidth. Matching to 50 Ohms within the maximum achievable bandwidth.

- Radiation pattern. In order to communicate with a sensor array located around the waist, an omnidirectional pattern is preferred.

According to the aforementioned requirements, conical skirt monopoles or discone antennas could be a good candidate owing to its really compact structure, its omnidirectional radiation pattern within the entire frequency range and its large bandwidth. This kind of antenna is widely used within UWB frequency band [73], [74]. Hence, these kinds of antennas could comply with the requirements of the WCE. 


\subsubsection{Discone Antenna Features}

As mentioned in section Section 3.2.1.2, discone antennas can be a fair candidate as UWB antenna for the WCE. They have a physical structure according to the hemispherical part of the WCE. Besides, their radiation pattern is omnidirectional within the entire frequency range and presents a large bandwidth.

Discone antennas are a monopole. These are a variation of the biconical one adding a small disc as ground plane. They can be considered a kind of modified dipole. These modifications are often used to obtain wide bandwidths without increasing complexity. Thus, these antennas can be manufactured in a non-complex way. The discone antenna consists of a circular disc and a cone. The disc is typically smaller than the maximum diameter of the cone (see Figure 3.6). The tip of the cone is frequently truncated and the antenna fed between the tip of the cone and the disc. The cone is often capped to improve performance. The upper part of the cone is usually flared more than the remainder of the cone to decrease the cone height. Precise construction of the area near the feed is important since this region determines the high frequency performance. The discone antenna is generally fed by means of a coaxial cable running through the cone.
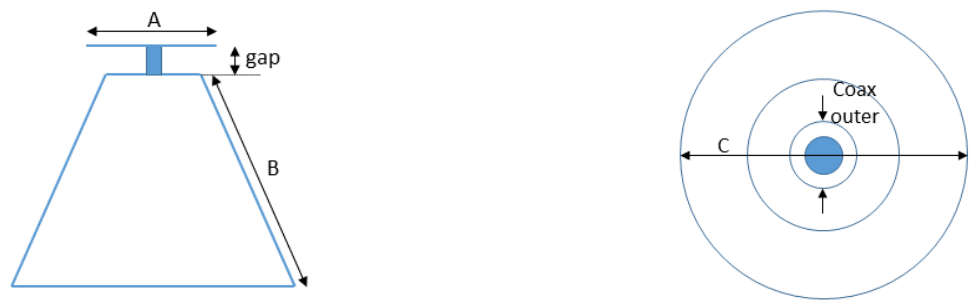

Figure 3.6: Discone antenna parameters.

According to Figure 3.6, each part of the antenna is parameterized as:

- $\mathrm{A} \Rightarrow$ Ground plane diameter.

- $\mathrm{B} \Rightarrow$ Side length of the cone.

- $\mathrm{Gap} \Rightarrow$ Spacing between disc and conical structure.

- $\mathrm{C} \Rightarrow$ Maximal diameter of the cone.

- Coax outer $\Rightarrow$ Minimal diameter of the cone. 
The main characteristics to be considered to optimize the model are the following [75]:

- Decreasing the whole antenna structure, the operating frequency can be increased.

- In order to mitigate the ripples on the reflection coefficient, increase the cone diameter $\mathrm{C}$.

- In order to vary the input impedance, increase/decrease the flare angle (angle between the center and the side of the cone).

Apart from these characteristics, it should be highlighted that the dielectric features of human tissues can produce modifications in the initial antenna design.

\subsubsection{Antenna Design}

\section{Free Space Validation}

Considering the dimensions of the discone antenna described in Section 3.2.1.3, the first antenna design has been optimized to work at higher frequencies. It should be mentioned that the coaxial cable which feeds the antenna has been considered from this starting stage. Thus, more realistic results are obtained. The chosen coaxial cable is a $50 \Omega$ RG405 whose diameters of the inner and outer conductors are $0.51 \mathrm{~mm}$ and $1.68 \mathrm{~mm}$, respectively. This cable has been selected since the final antenna design has to be really tiny due to the size of the capsule.

Computer Simulation Technology $\left(\mathrm{CST}^{\circledR}\right)$ software has been chosen for the analysis of the antenna designs. After a parametric sweep of simulations, the best antenna matching within $20-30 \mathrm{GHz}$ has been obtained with the following dimensions:

- $\mathrm{A}=10.25 \mathrm{~mm}$

- $\mathrm{B}=10.91 \mathrm{~mm}$

- $\mathrm{C}=12.99 \mathrm{~mm}$

- $\operatorname{gap}=0.5 \mathrm{~mm}$

- coax_outer $=1.68 \mathrm{~mm}$ 
The antenna model in free space is shown in Figure 3.7.

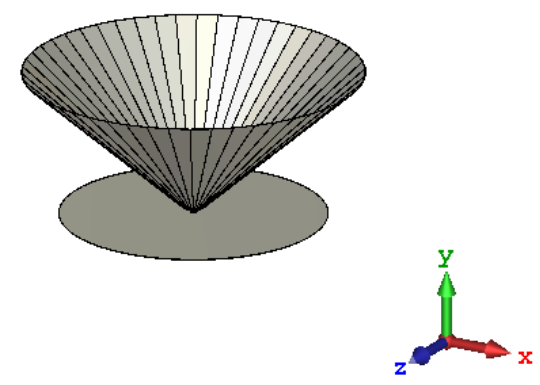

Figure 3.7: Discone antenna model in free space.

The return loss is depicted in Figure 3.8. As can be observed, the resonance frequency is around $25 \mathrm{GHz}$ and the antenna has a $-10 \mathrm{~dB}$ bandwidth of 10 $\mathrm{GHz}$, approximately. Regarding the input impedance of the antenna in free space, the real part is close to $50 \Omega$ from 23 to $28 \mathrm{GHz}$ and the imaginary part is quite low within these frequencies (see Figure 3.9 and Figure 3.10).

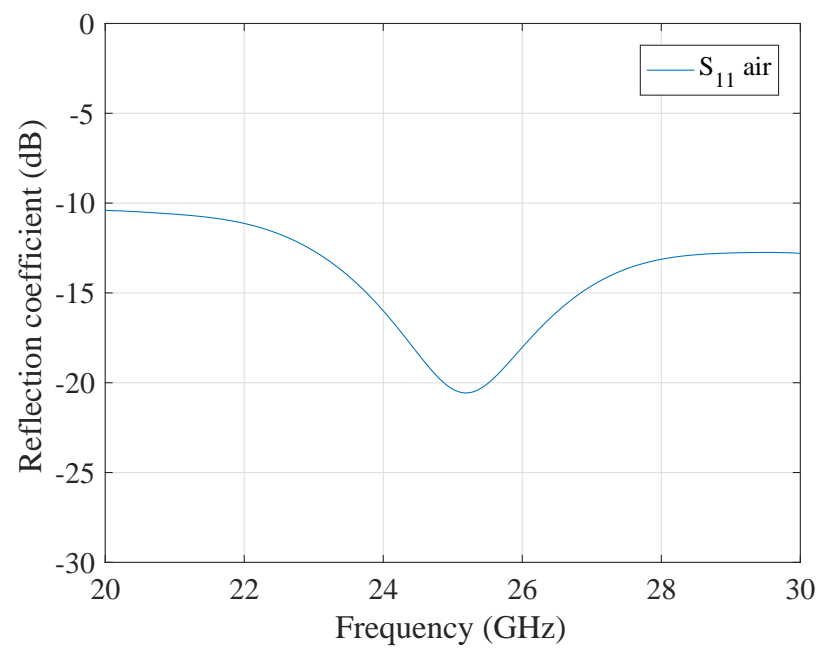

Figure 3.8: Reflection coefficient of discone antenna designed to work at high frequencies (Free space). 


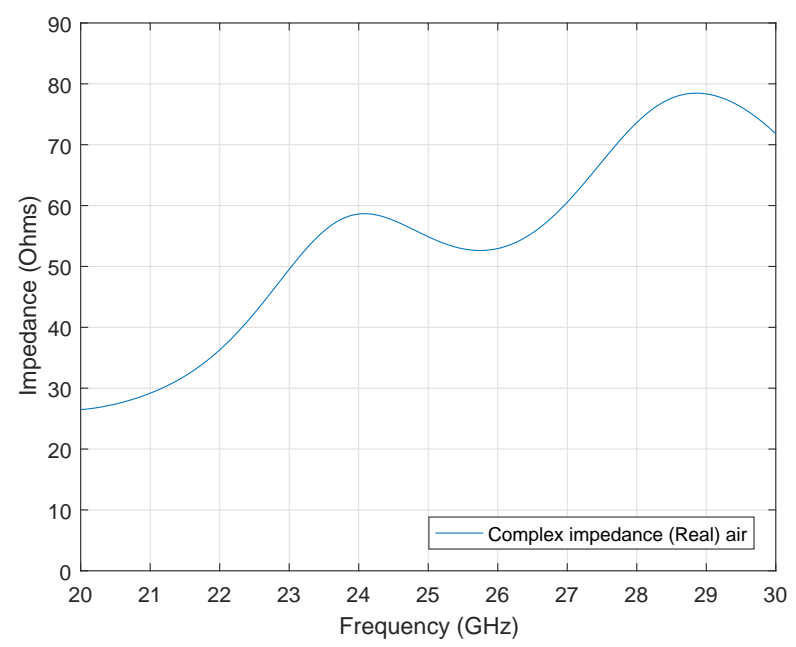

Figure 3.9: Real part of the input impedance of discone antenna (Free space).

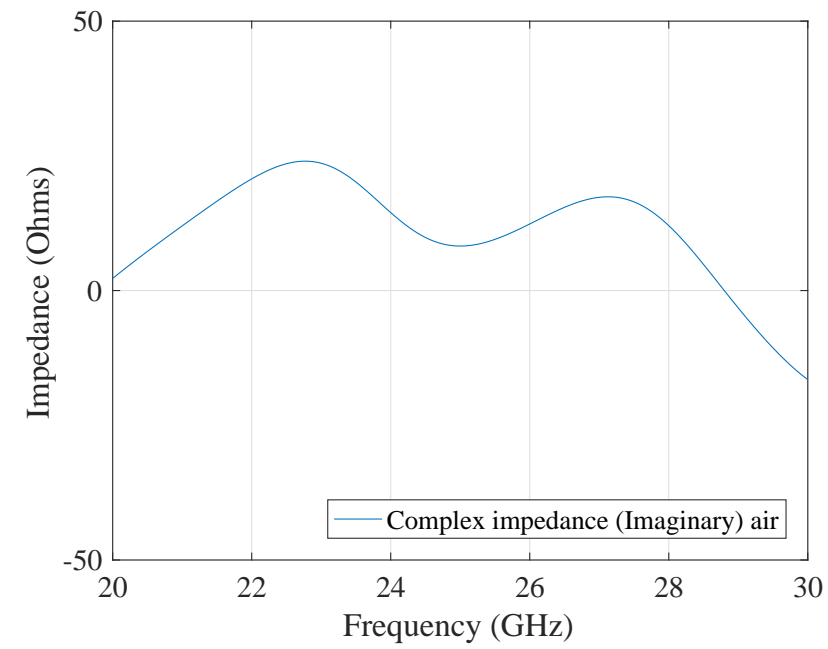

Figure 3.10: Imaginary part of the input impedance of discone antenna (Free space). 

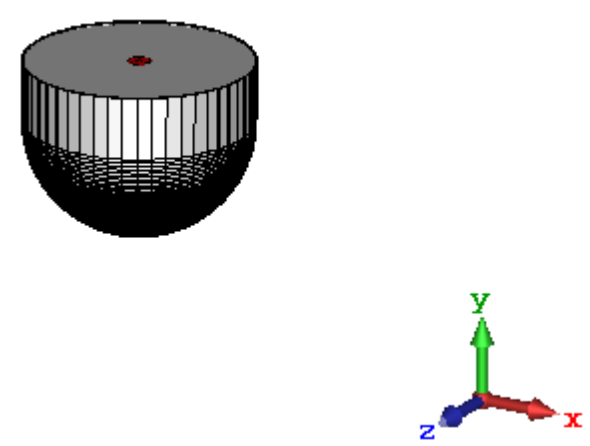

Figure 3.11: Discone antenna embedded into the capsule endoscope.

\section{Simulation Setup}

In simulation, one of the most relevant challenges in order to reproduce a realistic in-body scenario is the setup. In our particular case, the antenna should be embedded in a WCE. Therefore, the shape of the capsule as well as the material are attempting to be reproduced. According to Figure 3.5, the antenna is embedded in the upper part of the capsule which has a hemispherical shape. The external material is polyethylene with a thickness of $0.1 \mathrm{~mm}$, approximately. Taking into account the aforementioned comments, the whole structure has been reproduced in the simulation software as can be seen in Figure 3.11. It is worth mentioning that the inner part of the capsule is filled with air as in real conditions.

Apart from the WCE, the human tissue layers should be included as well. As mentioned in Section 2.2.1, the muscle tissue has been considered a fair approximation of the human abdominal region. Hence, the capsule is submerged in a brick which has the dielectric properties of the human muscle tissue (see Figure 3.12).

At this point, it can be noted that the antenna model for free space is not small enough to be embedded in the upper part of the dome of the capsule. Nevertheless, it is important to know what is the effect of both added layers in the radiation parameters. Therefore, the diameter of the capsule has been enlarged up to the diameter of the discone antenna (see Figure 3.13). Thus, a first approximation is obtained. The return loss considering both layers is shown in Figure 3.14. As can be seen, the resonance frequency shifts down 

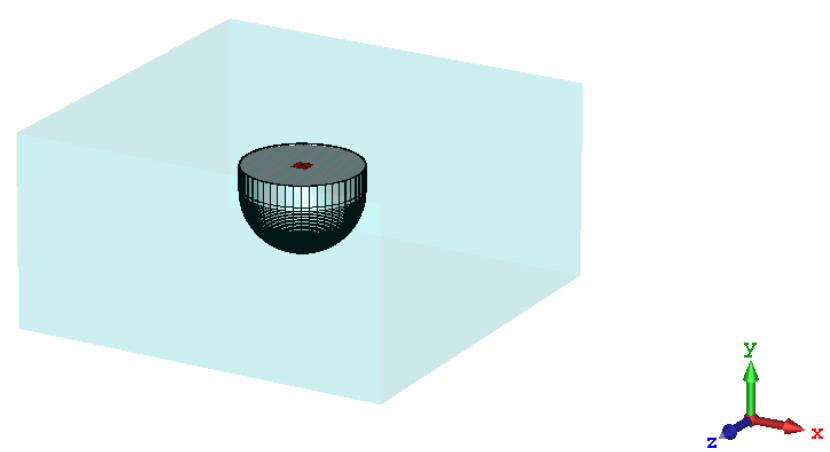

Figure 3.12: Capsule endoscope and antenna wrapped with the phantom layer.

considerably compared to that of free space. This is by the SF explained in Section 3.2.1.1. Here, the resonance frequency is around $10 \mathrm{GHz}$.

So, there are two main issues in order to get a proper discone antenna model for the WCE. On the one hand, the antenna should be resized in order to be embedded in the dome of the capsule. On the other hand, the resonance frequency should shift down where the losses of the propagation medium are lower.

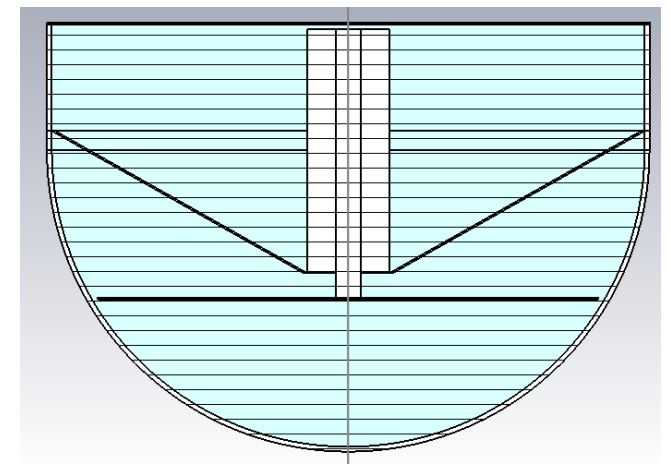

Figure 3.13: XY cross section of the discone antenna and capsule. 


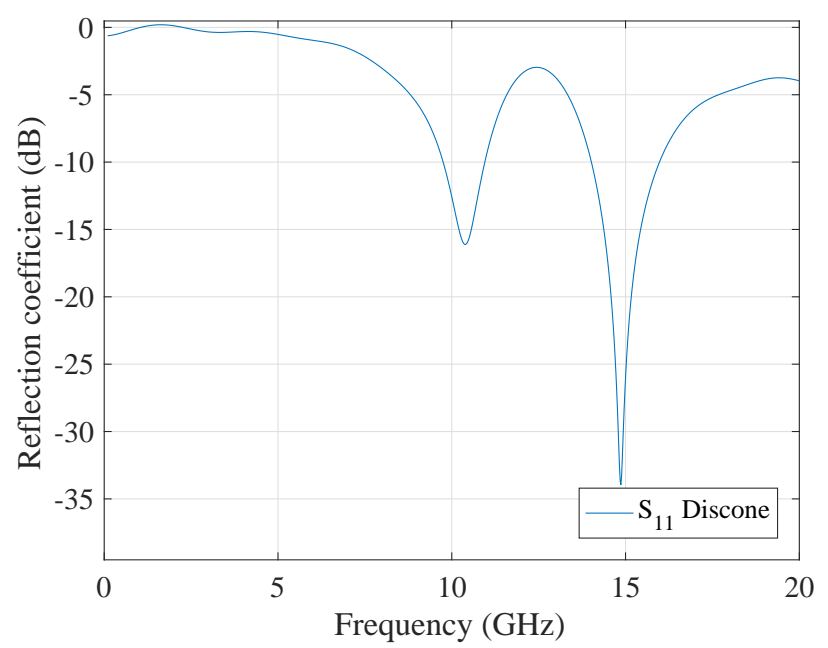

Figure 3.14: Reflection coefficient of the embedded antenna into the capsule endoscope and wrapped with the phantom layer.

\section{Resizing and Optimization of the Antenna Model in Free Space}

As mentioned in the previous section, the antenna should be resized in order to fit into the capsule. Introducing some steps in the conical structure could be an optimization technique to resize the antenna [74]. In this way, the current flow in the side of the cone is kept. Besides, as mentioned in [74], the height of these steps can vary the resonance frequency. Nevertheless, the maximal conical radius would not vary its diameter and should be decreased. As mentioned in Section 3.2.1.3, this could affect the input impedance of the antenna.

The use of magneto-dielectric composites is other solution widely used in order to reduce the size of the antenna. The technique consists in filling the antenna with a ferrite (see Figure 3.16). Thus, the antenna can be resized, i.e., miniaturized. 

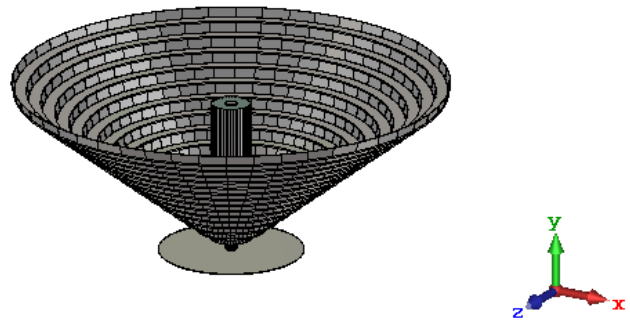

Figure 3.15: Discone antenna model with 15 steps.
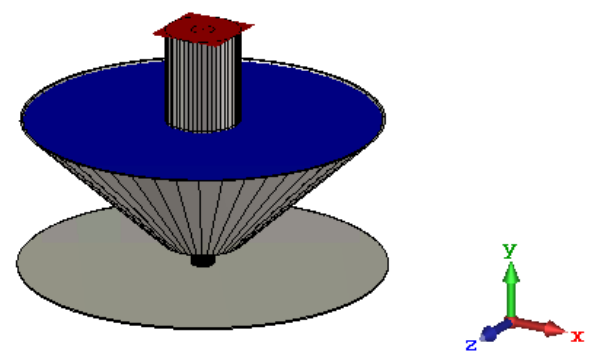

Figure 3.16: Discone antenna filled with ferrite.

After several simulations including a ferrite material with a dielectric permittivity of 5 , the resonance frequency is shifted down and the antennas has a overall size to be embedded:

- $\mathrm{A}=8.25 \mathrm{~mm}$

- $\mathrm{B}=2.91 \mathrm{~mm}$

- $\mathrm{C}=7.99 \mathrm{~mm}$

- gap $=0.51 \mathrm{~mm}$

Although the antenna is miniaturized, the resonance frequency only shifted down by $0.3 \mathrm{GHz}$ and the overall size is not small enough for the WCE. Besides, the bandwidth is narrower than without this dielectric material. 
Other technique used in low frequencies to reduce the antenna size consists in using a back cavity that surrounds the cone of the antenna [76]. Using this technique, a really compact structure maintaining the current flow path can be achieved. Accordingly, this technique is applied here to assess its performance at high frequencies for this specific purpose.

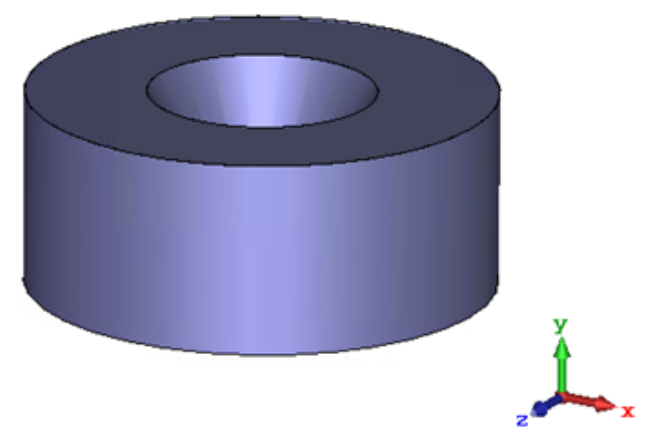

Figure 3.17: Discone antenna optimized by back cavity technique

After resizing and optimizing the antenna with the back cavity, a suitable overall size for the WCE is achieved (see Figure 3.17). The new antenna dimensions are the following:

- $\mathrm{A}=4.25 \mathrm{~mm}$

- $\mathrm{B}=2.96 \mathrm{~mm}$

- $\mathrm{C}=2.42 \mathrm{~mm}$

- Gap $=1 \mathrm{~mm}$

- Radius of upper circle $=3.5 \mathrm{~mm}$

- Height of external cylinder $=2.96 \mathrm{~mm}$

As can be seen in Figure 3.18, the antenna is tuned within the low part of UWB frequency range. The bandwidth is $1.04 \mathrm{GHz}$, approximately.

Regarding the input impedance, in Figure 3.19, one can observe that the real part of the impedance is lower than $50 \Omega$. This decrease in the real part of the impedance can be produced by the lossy propagation medium along with 
the tiny dimensions of the antenna. Therefore, a matching network between the antenna and the coaxial cable would be necessary.

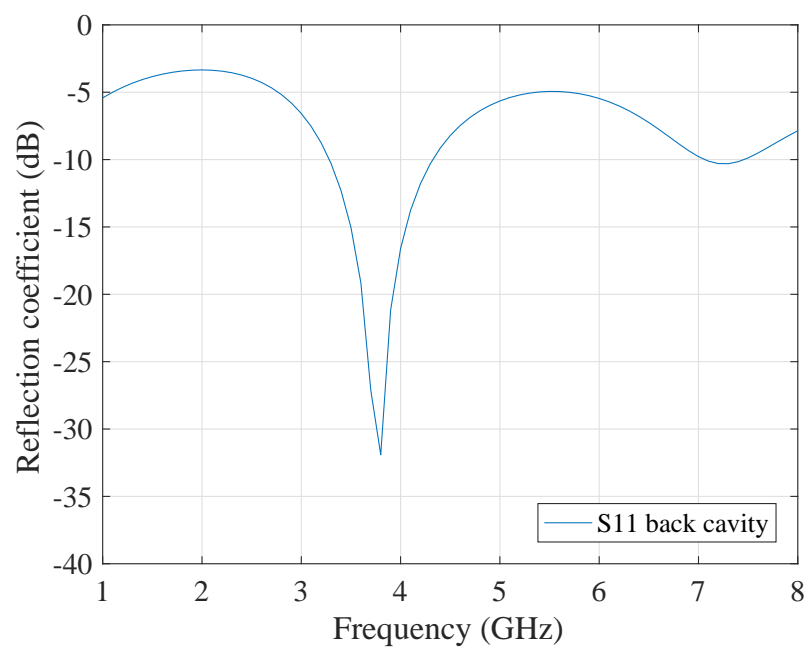

Figure 3.18: Reflection coefficient of the discone antenna optimized by back cavity technique

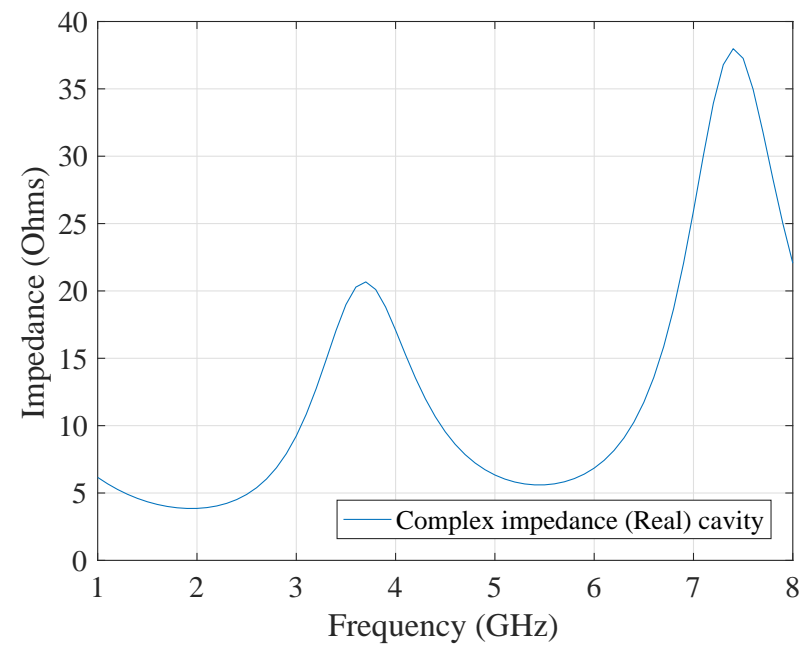

Figure 3.19: Real part of the input impedance of the discone antenna optimized by back cavity technique 


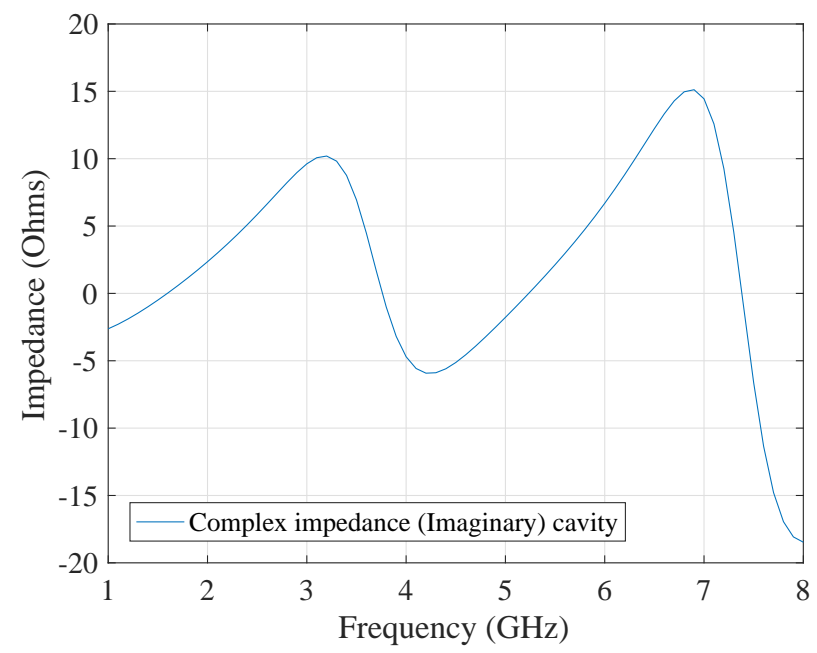

Figure 3.20: Imaginary part of the input impedance of the discone antenna optimized by back cavity technique

\section{Improvement of Input Impedance. Including a Short Wall}

As noted, the overall size of the antenna and the lossy propagation medium can be critical points as regard to the input impedance of the antenna. Considering the input impedance values obtained with the back cavity technique depicted in Figure 3.19, a matching network should be considered in order to keep $50 \Omega$ within the bandwidth. Furthermore, matching networks can improve the bandwidth as well. The main issue of matching network designs is that this is not a methodical process and usually is based on trial and error method [77]. Besides, this matching network should be included in the antenna model so that the overall size would be larger. Therefore, other techniques should be considered to increase the input impedance maintaining the large bandwidth achieved. One of this technique could be the inclusion of a short wall. In this particular case, the short wall would be connected between the conical structure and the ground plane. In Figure 3.21, the discone antenna with a short wall is depicted. 


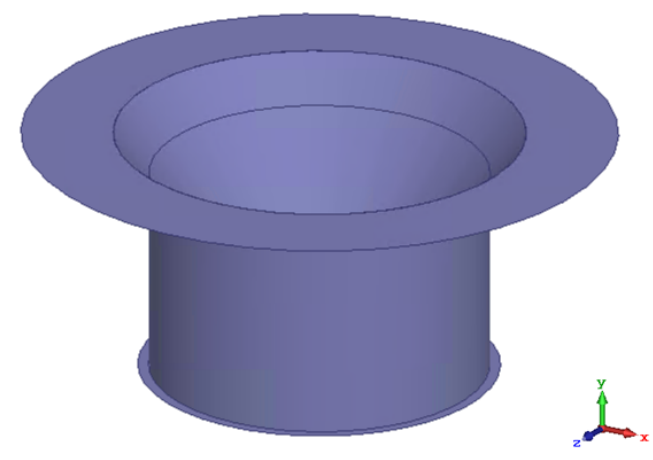

Figure 3.21: Discone antenna optimized by back cavity technique and short wall

As mentioned before, the main goal consists in keeping the large bandwidth as well as achieving an input impedance of $50 \Omega$ within the whole bandwidth. Including the short wall, the dimensions of the antenna are:

- $\mathrm{A}=7.99 \mathrm{~mm}$

- $\mathrm{B}=3.91 \mathrm{~mm}$

- $\mathrm{C}=4.25 \mathrm{~mm}$

- $\mathrm{Gap}=4.25 \mathrm{~mm}$

- Radius of upper circle $=3.5 \mathrm{~mm}$

- Height of external cylinder $=2.96 \mathrm{~mm}$

- Radius of short wall $=2 \mathrm{~mm}$

In Figure 3.22 and Figure 3.23, the return loss and input impedance of the antenna with back cavity and short wall is shown. As can be observed, the input impedance increases by including the short wall. Regarding the antenna matching, it is kept within the low part of UWB frequency band. 


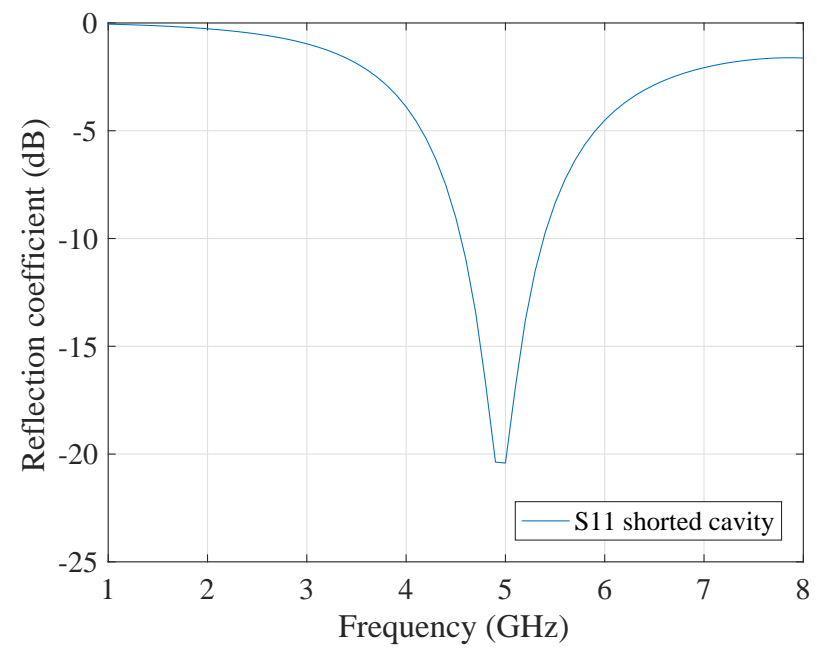

Figure 3.22: Reflection coefficient of the discone antenna optimized by back cavity technique and short wall

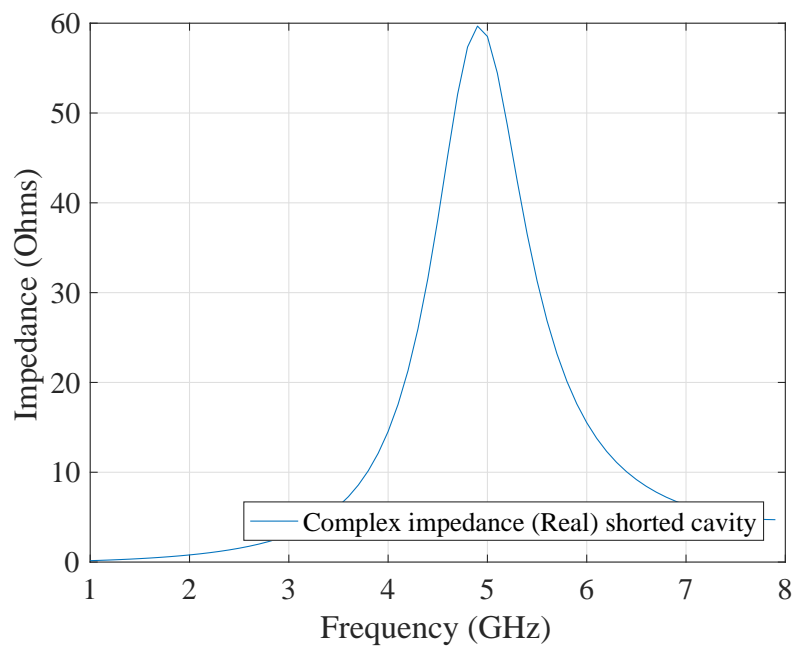

Figure 3.23: Real part of the input impedance of the discone antenna optimized by back cavity technique and short wall 


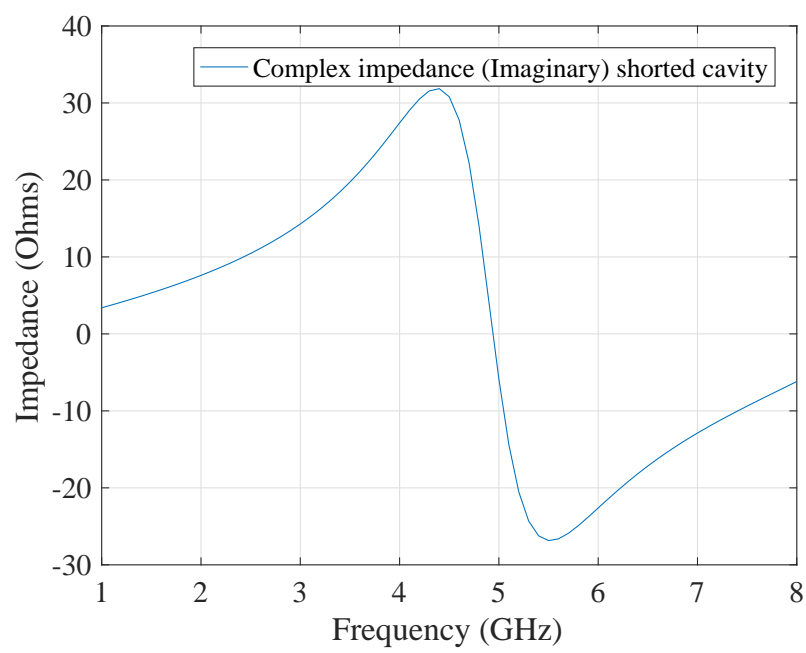

Figure 3.24: Imaginary part of the input impedance of the discone antenna optimized by back cavity technique and short wall

\section{Back Cavity Discone Antenna with Short Wall. Lossy Materials}

At this point, it is important to consider the materials which the antenna will be manufactured. The antenna model shown in Figure 3.21 is designed with a Perfect Electrical Conductor (PEC) surface. This PEC layer has been replaced by a copper layer with $1 \mathrm{~mm}$ of thickness (see Figure 3.25). Besides, the losses in the material has been taken into account as well. It should be mentioned that this change in the material could vary both the return loss and the input impedance. In order to keep the bandwidth and the impedance achieved with the last antenna model, a parametric sweep is performed. Afterwards, the discone antenna reached the following size parameters:

- $\mathrm{A}=8.6 \mathrm{~mm}$

- $\mathrm{B}=3.91 \mathrm{~mm}$

- $\mathrm{C}=6.84 \mathrm{~mm}$

- $\operatorname{gap}=1.5 \mathrm{~mm}$

- Radius of upper circle $=3.5 \mathrm{~mm}$ 
- Height of external cylinder $=2.96 \mathrm{~mm}$

- Radius of short wall $=2 \mathrm{~mm}$

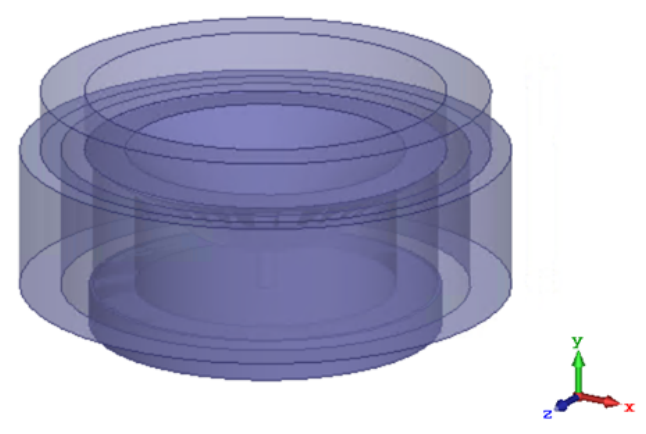

Figure 3.25: Discone antenna optimized by back cavity technique and short wall including a copper layer of $1 \mathrm{~mm}$ of thickness.

As can be seen in Figure 3.26 and Figure 3.28, a bandwidth of $1 \mathrm{GHz}$ and a impedance really close to $50 \Omega$ is achieved with the realistic antenna model.

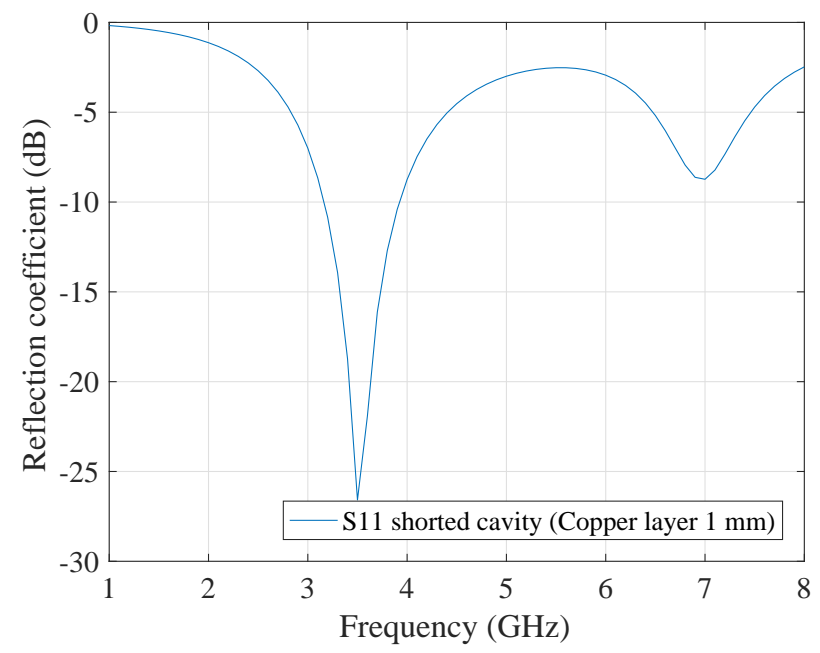

Figure 3.26: Reflection coefficient of the discone antenna optimized by back cavity technique and short wall including a copper layer of $1 \mathrm{~mm}$ of thickness 


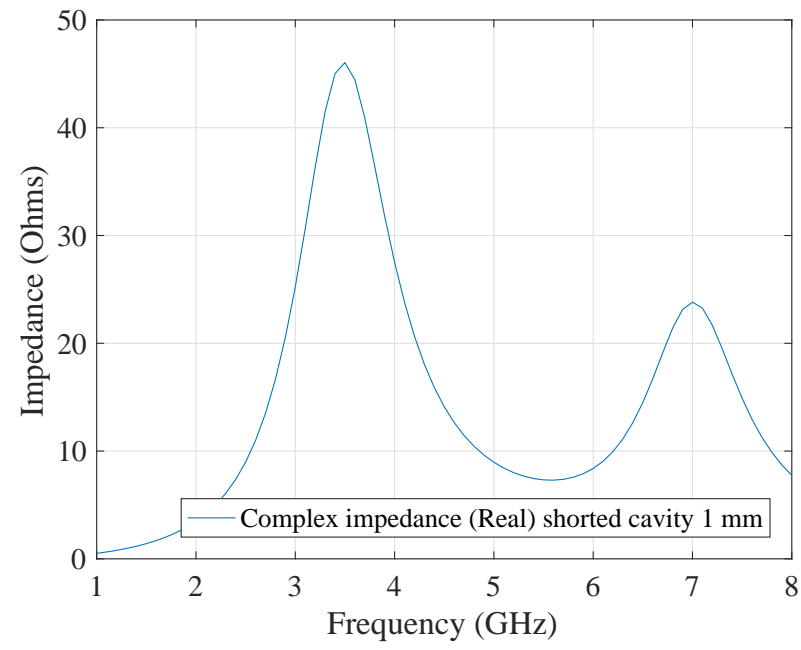

Figure 3.27: Real part of the input impedance of the discone antenna optimized by back cavity technique and short wall including a copper layer of $1 \mathrm{~mm}$ of thickness

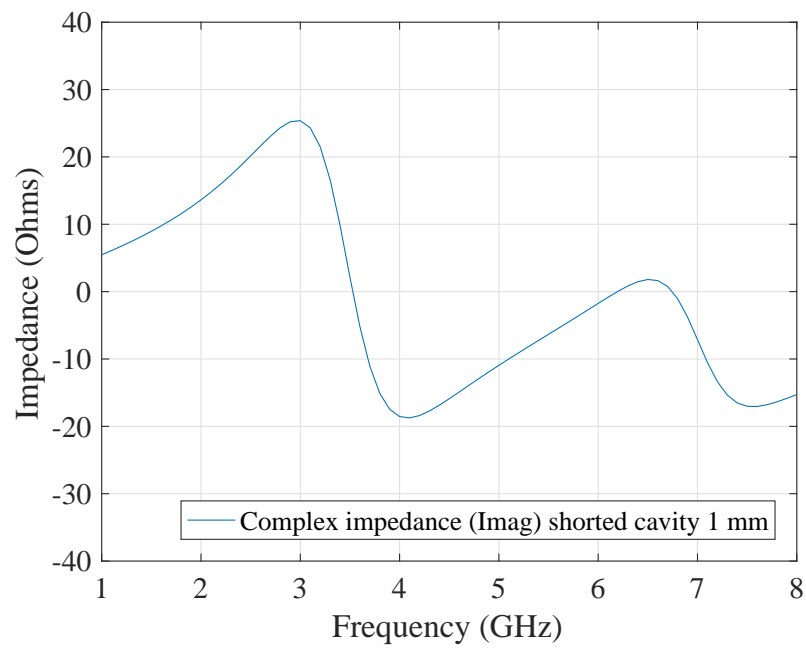

Figure 3.28: Imaginary part of the input impedance of the discone antenna optimized by back cavity technique and short wall including a copper layer of $1 \mathrm{~mm}$ of thickness 
After designing the discone antenna to work at high frequencies, a large number of trials by using different optimization techniques have been performed without success. Including steps on the structure or ferrite materials with different dielectric features have not given the expected results. Finally, a bandwidth of, approximately, $1 \mathrm{GHz}(700-800 \mathrm{MHz})$ within the low part of UWB spectrum with an input impedance close to $50 \Omega$ is achieved with the back cavity technique with a short wall.

\section{Manufactured Antenna. Validation}

Once the antenna complies with the requirements in terms of size and radiation parameters, it can be manufactured. The manufacturing process as well as the antenna validation was performed on the Technische Universität Dresden facilities. Due to the tiny dimensions of the antenna structure, it has been made by a 3D printer. 3D printers can produce a large number of different pieces with high accuracy. Currently, the limitation of these devices come from the materials that they can use to print each layer. For this reason, the antenna was manufactured with a material which is quite similar to the copper and is available to be used in a 3D printer. The chosen material is a kind of aluminium. In Figure 3.29, the manufactured antenna model is shown.

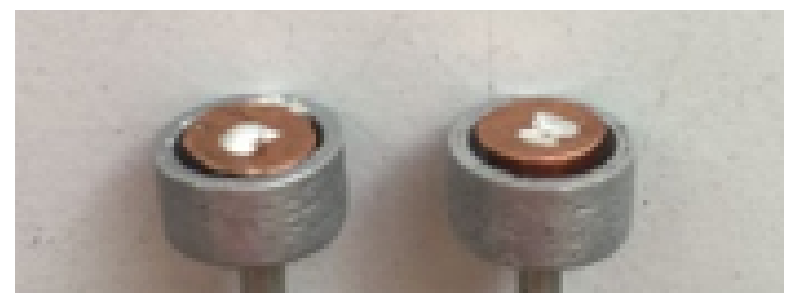

Figure 3.29: Manufactured discone antennas.

To reproduce the considered measurement setup faithfully, the antenna has been inserted in a polyethylene capsule (see Figure 3.31). Afterwards, the structure has to be submerged into the liquid phantom. Therefore, the capsule is sealed with a blue tack. Finally, the antenna is introduced in a bucket where the liquid phantom is poured. The phantom used is a sucrose solution $\left(C_{12} \mathrm{H}_{22} \mathrm{O}_{11} / 1.0 \mathrm{M}\right)$ that emulates the electromagnetic behaviour of the human muscle tissue with certain accuracy at UWB frequencies [40]. In Figure 3.30, the relative permittivity of prepared phantom is compared with the reported measured values for the real muscle tissue reported in [12]. 


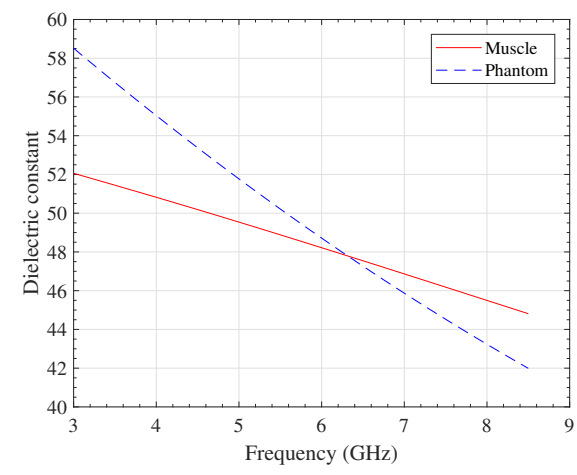

(a)

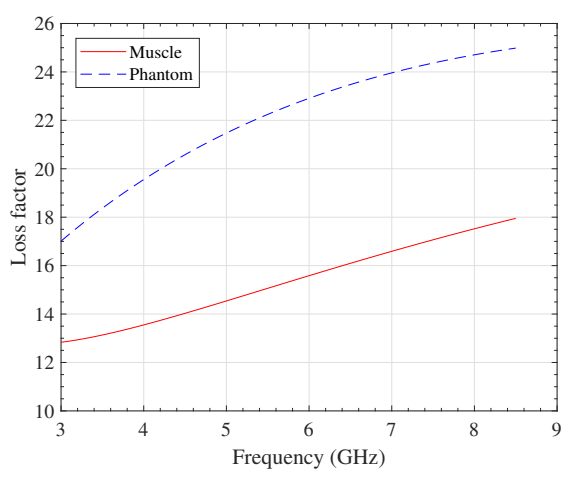

(b)

Figure 3.30: (a) Dielectric constant and (b) loss factor values of the phantom described in [40] and the real values of the human muscle tissue [12].

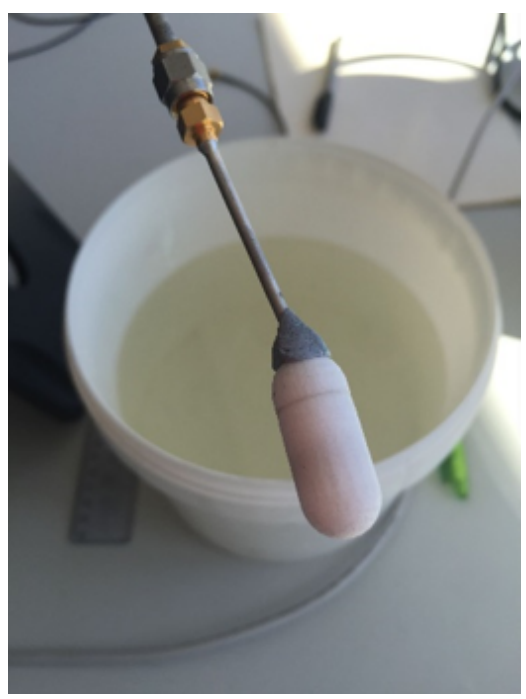

Figure 3.31: Discone antenna embedded into a polyethylene capsule.

A Keysight Fieldfox Network Analyzer is used. The Network Analyzer is calibrated through a full-port calibration from 0 to $25 \mathrm{GHz}$. The antenna is connected to the port 1 of the Fieldfox Network Analyzer. The measured reflection coefficient and the input impedance are depicted in Figure 3.32 and Figure 3.33, respectively. As can be observed, the reflection coefficient is below 
$-10 \mathrm{~dB}$ in the low part of the UWB spectrum (from 3 to $5 \mathrm{GHz}$ ). On the other hand, the resistance is close to $50 \Omega$ within this frequency range and the reactance is checked to be close to 0 .

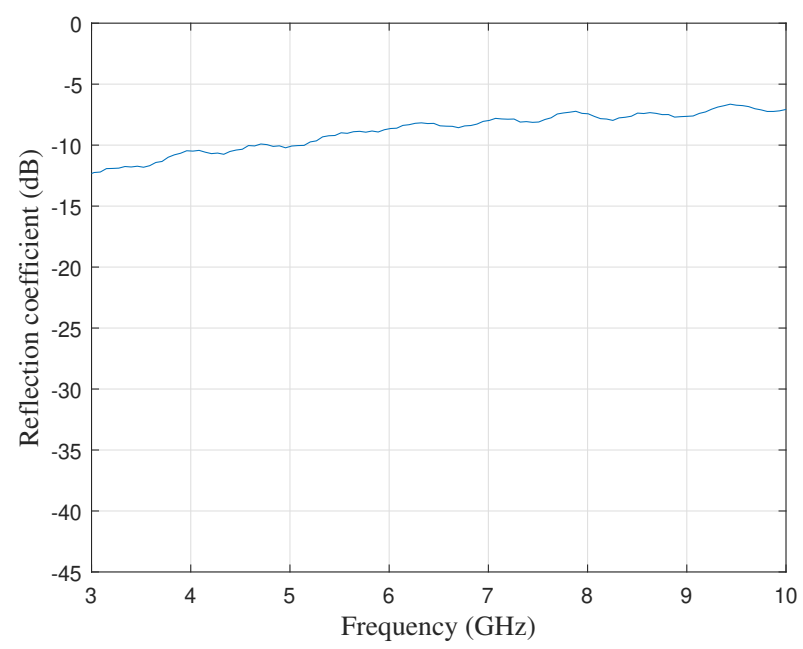

Figure 3.32: Reflection coefficient of manufactured antenna

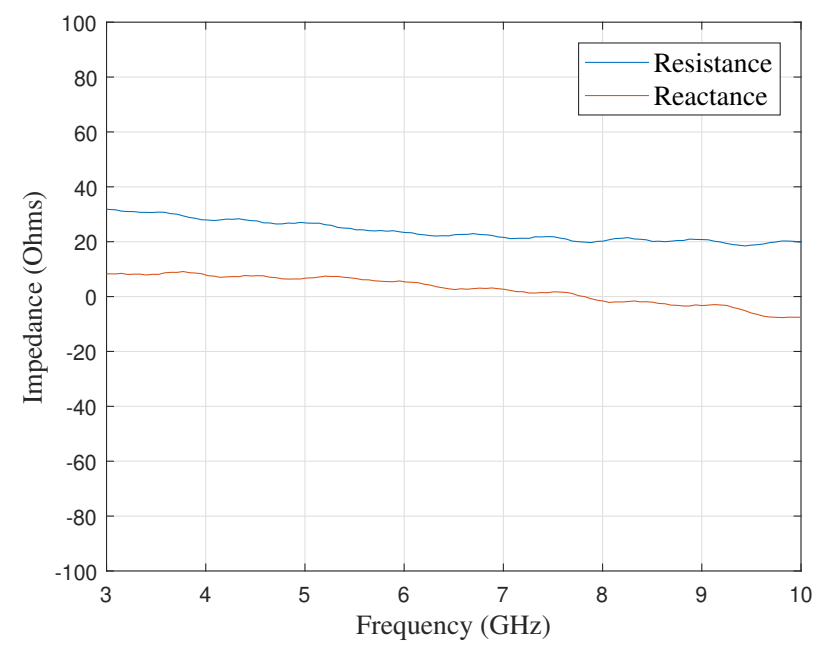

Figure 3.33: Real and Imaginary part of the input impedance of the manufactured discone antenna 


\subsubsection{Direct Antenna Design Procedure}

\subsubsection{Overview}

As mentioned in Section 3.2.1.1, most of works related to UWB implantable antennas apply the typical procedure for antenna miniaturization and optimization (see Figure 3.34). In this approach, the antenna is miniaturized in order to work at higher frequencies than UWB [33]. Then, the antenna is wrapped with a human body tissue which has a high dielectric constant. Thus, the bandwidth is shifted down since the wavelength is shorter [72]. However, the effectiveness of this approach may not be as high as expected in antennas with large bandwidth due to the frequency dependence of dielectric features of body tissues. As a consequence, a large bandwidth within the frequency range of interest could be unattainable. As seen in Section 3.2.1.1, it is quite complicated to optimize the antenna after being designed to work in free space. Besides, the bandwidth is not as wide as expected.

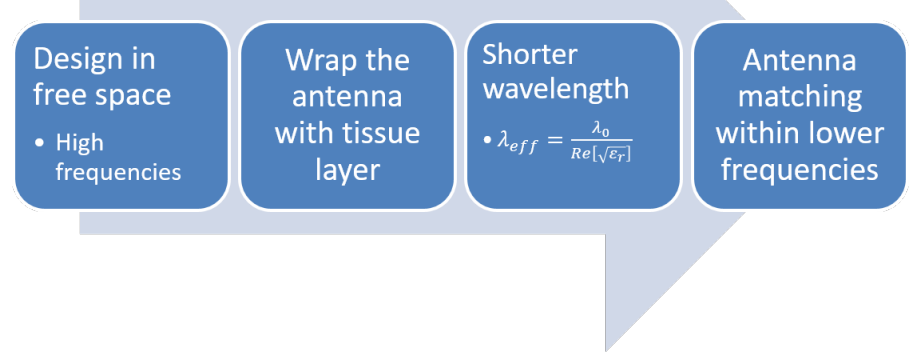

Figure 3.34: Most extended procedure for antenna optimization and miniaturization

In order to assess the UWB channel performance at the highest number of frequency points, the antenna matching should be as large as within this frequency range. In this section, a new optimization approach is presented (see Figure 3.35). Here, the in-body antenna is wrapped with a human tissue layer since the initial designing stage. Then, a miniaturization and optimization procedure is directly applied considering the real materials of the antenna. Concretely, a well-known antenna model is chosen and then it is miniaturized and optimized in order to keep the parameters that it has in free space. 


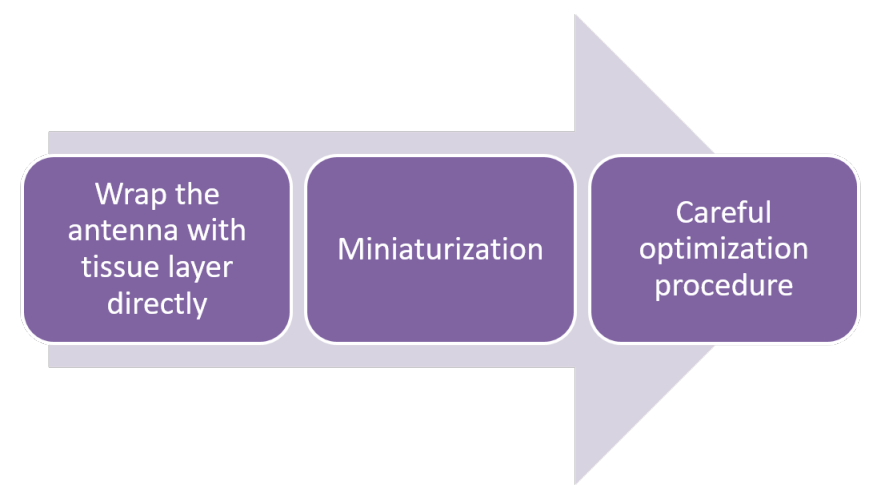

Figure 3.35: New approach of miniaturization and optimization. Direct antenna design technique.

\subsubsection{Simulation Setup \& Antenna Candidate}

Achieving high data rates occupying the whole UWB bandwidth is one of the most relevant challenges of UWB antennas. Besides, in-body antennas should have an omnidirectional radiation pattern at all frequencies to communicate with an on-body sensor array or an external receiver. Applying the direct antenna design procedure proposed here, a broadband and omnidirectional antenna is tried to achieve.

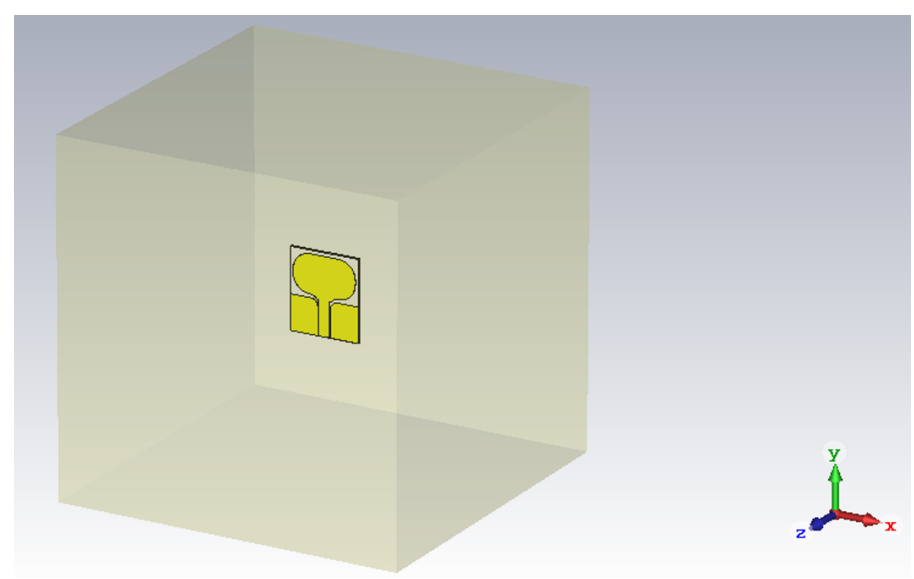

Figure 3.36: Candidate antenna wrapped with phantom layer. 


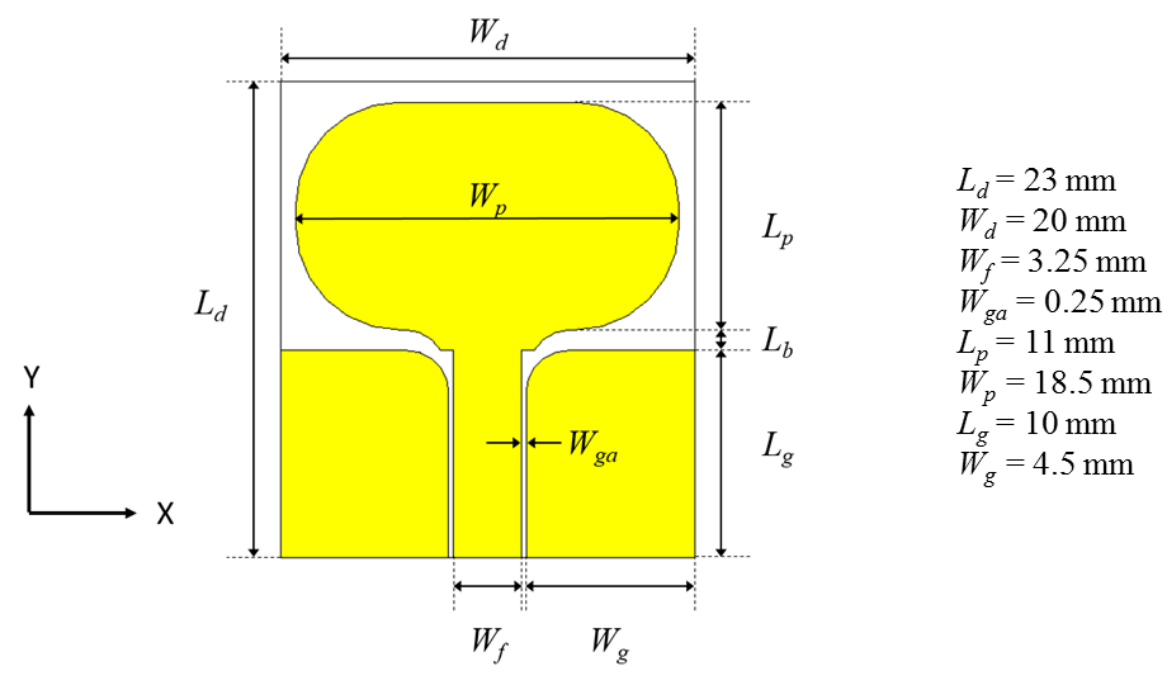

Figure 3.37: UWB optimized planar antenna dimensions.

As a first candidate to use this new designing approach, an UWB planar monopole with Coplanar Waveguide (CPW) feeding and circular patch is proposed. This kind of antenna presents a really compact structure, omnidirectional radiation pattern, linear polarization and broadband characteristics in free space [78]. As mentioned above, the antenna is wrapped with a human tissue layer since the initial designing stage (see Figure 3.36).

Instead of doing an antenna design with PEC material, lossy materials has been considered from the beginning to obtain more realistic conditions in the design stage. Both the copper and the dielectric layers have been included in the antenna model. As dielectric material, the Rogers 4003 substrate is chosen, which has a relative permittivity of 3.28 and a thickness of $0.813 \mathrm{~mm}$.

\subsubsection{Miniaturization \&3 Optimization Procedures}

As mentioned, the miniaturization and optimization procedures are carried out at the same time with this new approach. The overall size of the antenna is reduced, while several optimization techniques are considered in order to keep the characteristics that this kind of antenna has. The final antenna design is depicted in Figure 3.37. 
The bends in the ground plane are thoroughly studied in order to keep the omnidirectional radiation pattern when the antenna is implanted. Furthermore, a specific gap between the patch and the ground plane is carefully chosen in order to widen the bandwidth $\left(\mathrm{L}_{b}=1 \mathrm{~mm}\right)$. Moreover, the shape of the patch is elliptical in order to enlarge the bandwidth as well. This is in contrast to [78] where the patch is circular. The rest of antenna dimensions are depicted in Figure 3.37.

In Figure 3.38, the simulation in CST of the gain pattern in the XZ-plane is shown. The simulations are performed by considering the theoretical values of the permittivity for the human muscle tissue at UWB frequencies reported in [12]. From Figure 3.38, one can observe that the antenna exhibits a quasiomnidirectional radiation pattern in XZ-plane at UWB frequencies.

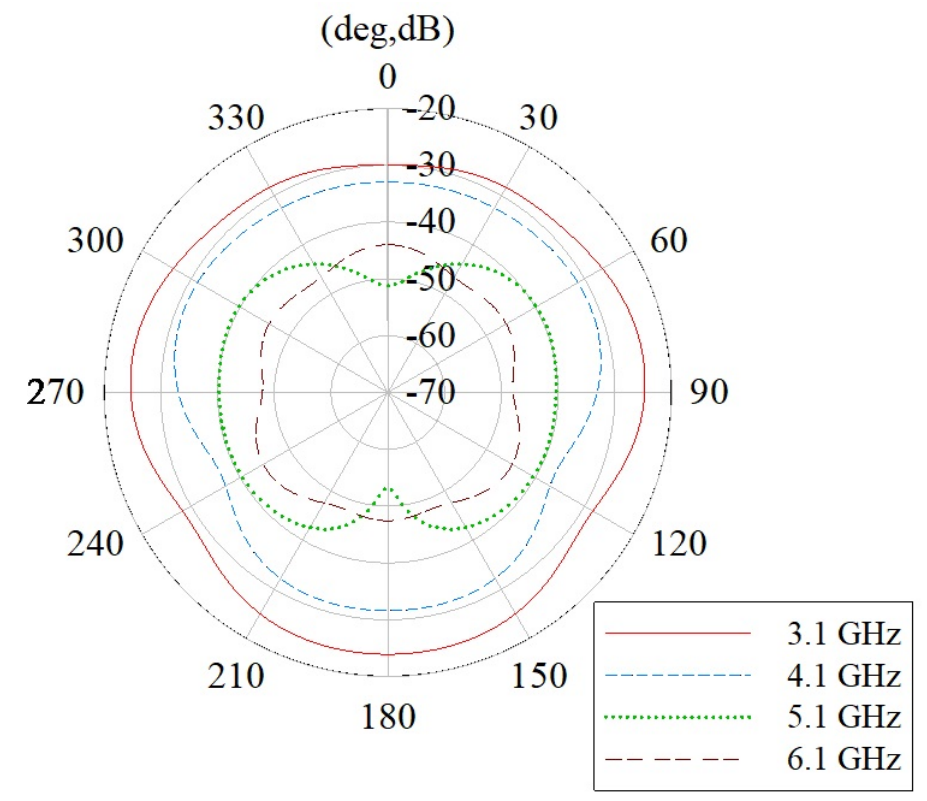

Figure 3.38: Gain pattern of the miniaturized antenna in the human muscle tissue at 3.1, 4.1, 5.1 and $6.1 \mathrm{GHz}$. 


\subsubsection{Testing}

The manufactured planar antenna presented in the previous section is depicted in Figure 3.39. This antenna was fabricated and measured at iTEAM research facilities.

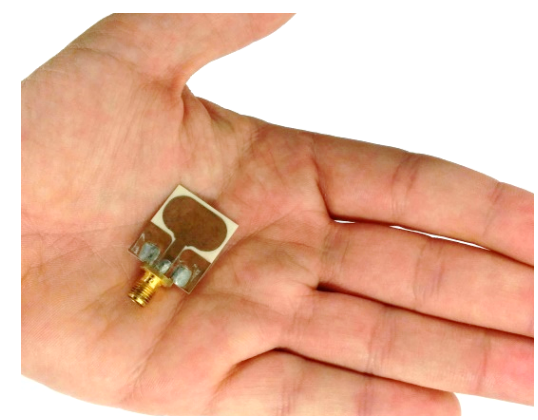

Figure 3.39: Manufactured UWB antenna which is miniaturized and optimized by the direct antenna design procedure.

Hereinafter, we refer to this antenna as the miniaturized antenna. After the manufacturing process, the antenna matching should be checked within the frequency band of interest. To validate the antenna working in an inbody environment, the sucrose liquid phantom described in [40] is used (see Figure 3.30).

In order to measure the return loss $\left(S_{11}\right)$, the antenna is submerged at a depth of $8 \mathrm{~cm}$ into the phantom which is poured into an extruded polystyrene foam container. The polystyrene container dimensions are $22 \times 22 \times 16 \mathrm{~cm}^{3}$ with a wall thickness of $40 \mathrm{~mm}$. The antenna has been previously covered and isolated with a layer of latex rubber to avoid the physical contact between the submerged antenna and the phantom in order to prevent a short-circuit of the printed board elements as well as to protect the antenna and connectors. Subsequently, the antenna is connected to an Agilent Technologies ${ }^{T M}$ ENA E5072A VNA. The VNA is calibrated through a full port calibration within the entire frequency band $3.1-8.5 \mathrm{GHz}$ in order to remove the cable effects.

The simulated and measured results for the $S_{11}$ are depicted in Figure 3.40. It is worth mentioning that the effect of the latex rubber which covers the antenna is practically negligible, since this layer has not been considered in the simulations and the curves are quite similar. It can be noted that the three curves exhibit a similar behaviour within all frequency points. Also, 
emphasize that all values are below $-10 \mathrm{~dB}$ in the three cases. Therefore, it can be concluded that the miniaturization approach proposed in this section keeps the antenna matching within the entire frequency band. In addition, it should be highlighted the great similarity between simulated and measured results.

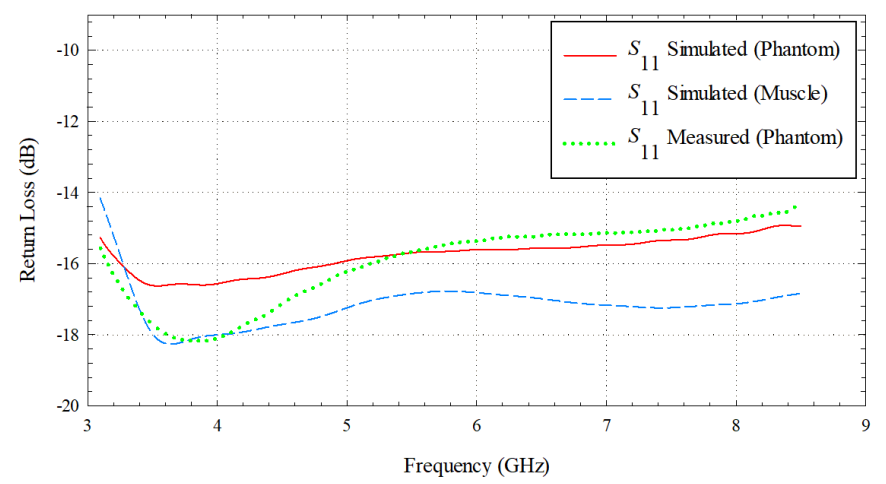

Figure 3.40: Measured and simulated return loss from 3.1 to $8.5 \mathrm{GHz}$

It should be pointed out that the miniaturized antenna has a low radiation efficiency due to the high losses of the surrounding medium (see Figure 3.41, Figure 3.42). These losses are in part responsible for the low $S_{11}$ values and for the broad matching bandwidth of the antenna. However, as will be shown in Section 3.2.2.6, the received power values are high enough to establish a communication channel in different scenarios despite the low efficiency of the miniaturized antenna. In any case, applying the direct antenna design procedure achieves a large bandwidth within the whole available bandwidth faster and more efficient than the conventional one explained in Section 3.2.1.1. Besides, the radiation pattern is kept with this technique. 


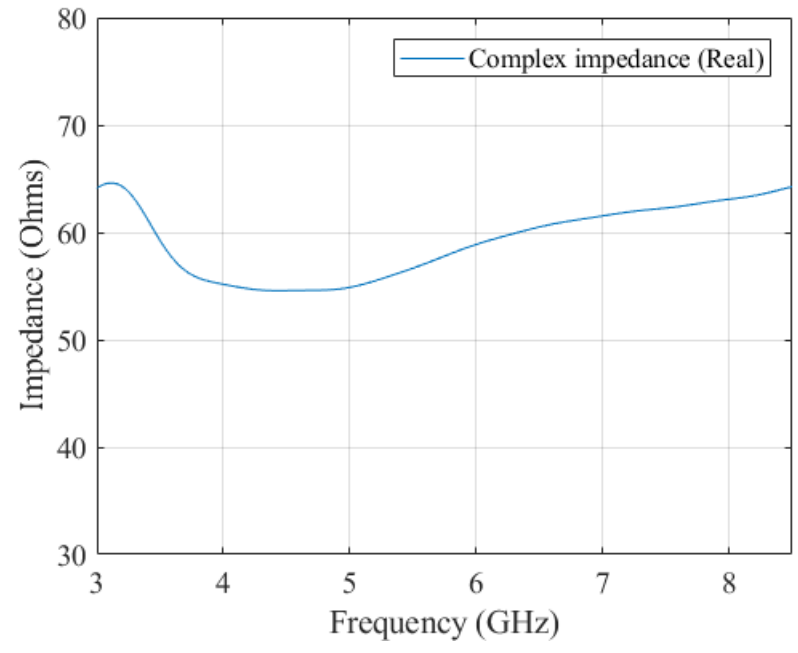

Figure 3.41: Real part of the input impedance of the miniaturized antenna.

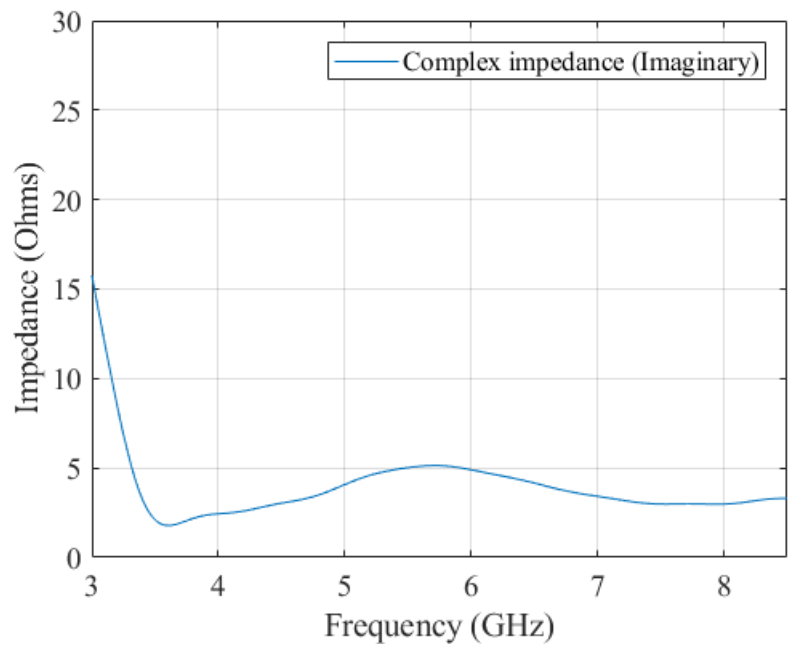

Figure 3.42: Imaginary part of the input impedance of the miniaturized antenna. 


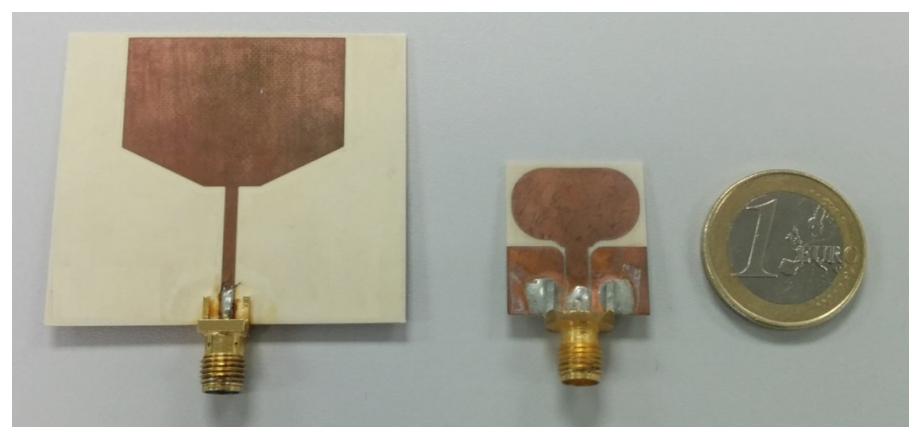

Figure 3.43: Transmitting and receiving UWB antennas.

\subsubsection{Experimental Propagation Measurement Setup}

In order to assess the direct miniaturization technique, the performance of the miniaturized antenna is compared with a larger UWB monopole used in [51] as implanted antenna. This UWB monopole has an overall size of $50 \times 40$ $\mathrm{mm}^{2}$. More details can be found in [79]. Hereinafter, we refer to this antenna as the large one. On the left-hand side of Figure 3.43, this large antenna is shown. Regarding the overall size of the miniaturized antenna in comparison with the large one, it has been reduced by a factor of nearly 2.2 times.

In order to evaluate the performance of the antennas, an IB2OB and an IB2OFF setups are considered. These two scenarios are emulated by using the setup depicted in Figure 3.44.

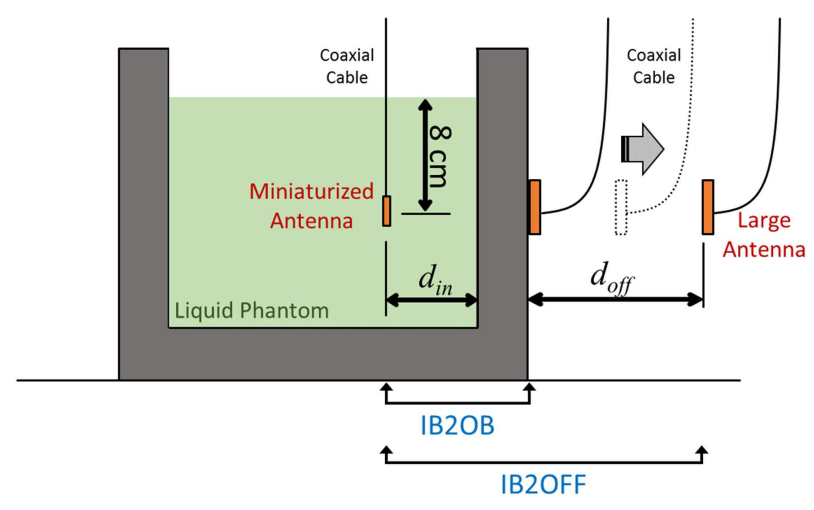

Figure 3.44: Measurement setups for each scenario. 
As mentioned above, the performance of the miniaturized antenna is assessed. To achieve this goal, the measurement campaign reported in [51] is replicated by changing the in-body antenna for our miniaturized antenna. That is, the inner antenna used in the setup depicted in Figure 3.44 is the miniaturized antenna, meanwhile in the same phantom-based setup described in [51] the large antenna is used as implanted antenna. In both cases, another large antenna with the same features is used as receiving antenna.

According to Figure 3.44, the liquid phantom is poured into the polyethylene container as in Section 3.2.2.4. In order to avoid the physical contact with the phantom, the implantable antenna is covered again with a layer of latex rubber and submerged into the phantom at a depth of $8 \mathrm{~cm}$. The external antenna is placed in a vertical position out of the container. In each scenario the antennas are properly oriented to achieve a co-polarized mode, i.e., the antennas are placed facing each other with the same polarization. The transmitting and receiving antennas are connected to port 1 and port 2 of the VNA, respectively.

Regarding the IB2OB scenario, the submerged antenna is moved away from the internal container face, whereas the on-body antenna is placed and fixed outside over the external container wall. The spacing between the internal container face and the inner antenna is $d_{i n}$. The thickness of the container wall is disregarded as proposed in [13]. The measurements are made with an initial antenna separation of $d_{\text {in }}=20 \mathrm{~mm}$, which is increased in steps of 10 $\mathrm{mm}$ up to $80 \mathrm{~mm}$ backwards in a straight line.

Considering the IB2OFF scenario, the submerged antenna is fixed to a distance of $d_{i n}=40 \mathrm{~mm}$ into the phantom, whereas the off-body antenna is moved away from the external container face. The distance between the external container face and the off-body antenna is $d_{\text {off }}$.

\subsubsection{Results}

From measurements, the forward transmission coefficient $\mathrm{S}_{21}(f, d, k)$ is obtained, where $f$ is the frequency value, $k$ is the snapshot number and $d$ is the distance between antennas. Before the data processing, $k=5$ snapshots are averaged in order to measure the Signal-to-Noise ratio (SNR). After averaging the samples, the $\tilde{S}_{21}(f, d)$ is obtained. The relative received power as a function of frequency for a given separation distance between antennas is computed as: 


$$
P(f, d)=\left|\tilde{S}_{21}(f, d)\right|^{2}
$$

\section{IB2OB}

Figure 3.45 depicts the relative received power obtained from each pair of antennas in the IB2OB scenario. From this figure, one can observe that the noise level is located at a received power around $-120 \mathrm{~dB}$.

From the results, it can be noted that the frequency responses of the channel are very similar, especially when the distance between antennas is lower than $50 \mathrm{~mm}$. On the one hand, the frequency drop is explained by the fact that the antenna gain falls down with the increment of frequency (see Figure 3.38). On the other hand, this drop is also related to the propagation medium, since the received power always decreases as the frequency raises [12].

As can be observed in Figure 3.45, the slope is steeper as the separation distance between antennas increases. For instance, using the large antenna for a separation distance of $d_{i n}=40 \mathrm{~mm}$, the received power decreases from -56.3 $\mathrm{dB}$ at $3.1 \mathrm{GHz}$ to $-70.7 \mathrm{~dB}$ at $4.1 \mathrm{GHz}$, whereas for a separation distance of $d_{\text {in }}$ $=50 \mathrm{~mm}$ it decreases from $-64.7 \mathrm{~dB}$ to $-85.1 \mathrm{~dB}$ in the same frequency points. In the same way, considering the miniaturized antenna, the power decreases

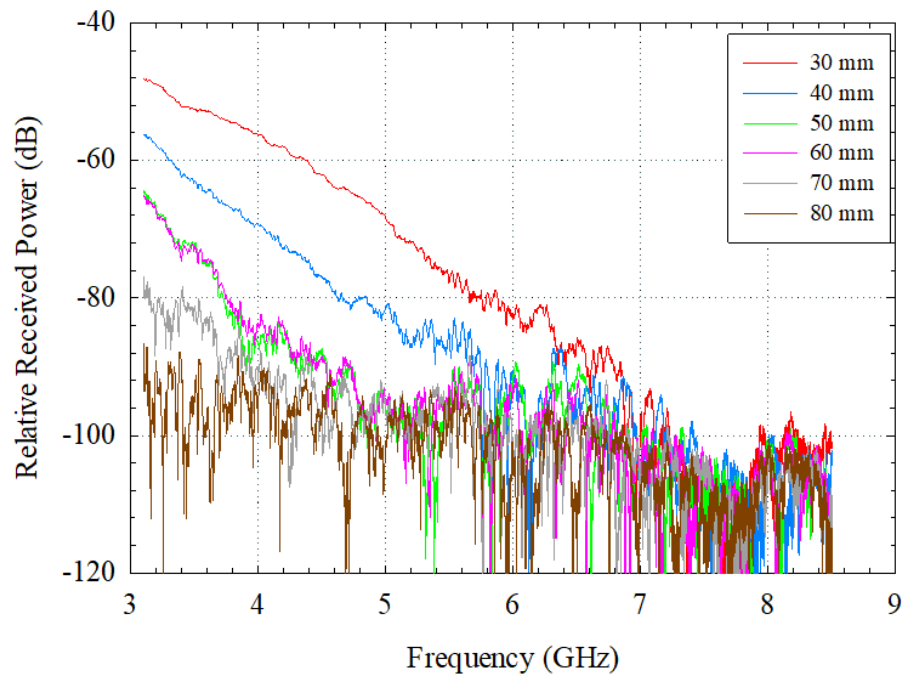

(a) 


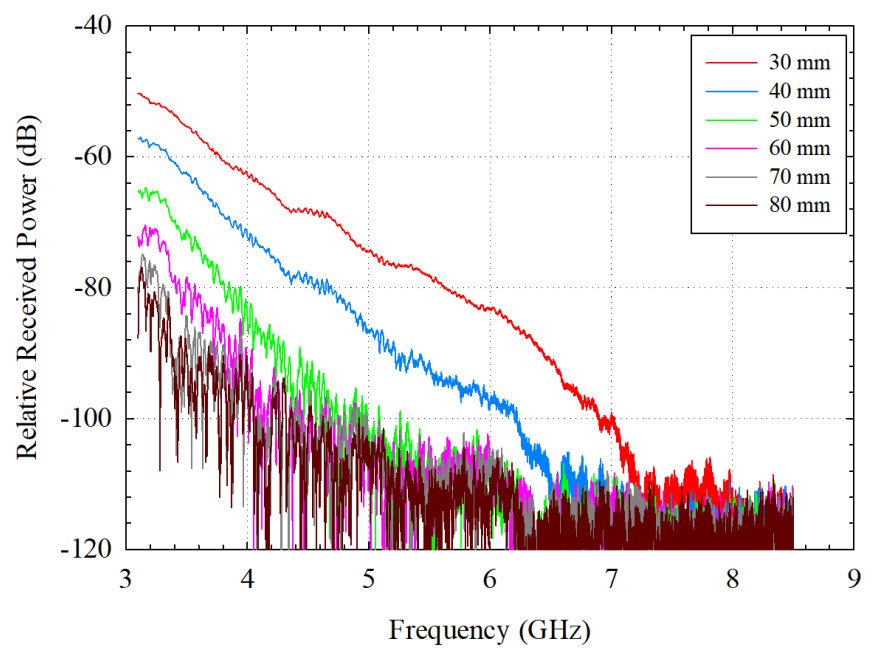

(b)

Figure 3.45: Relative received power as a function of the frequency in the IB2OB scenario for different values of $d=d_{\text {in }}$ when (a) the large and (b) the miniaturized antennas acted as transmitters.

from $-57.1 \mathrm{~dB}$ to $-73.6 \mathrm{~dB}$ for $d_{i n}=40 \mathrm{~mm}$ and from $-65.1 \mathrm{~dB}$ to $-86.3 \mathrm{~dB}$ for $d_{i n}=50 \mathrm{~mm}$. Therefore, comparing the power decay with frequency of each pair of antennas within the frequency band 3.1-4.1 GHz, a difference of $2.1 \mathrm{~dB}$ for $d_{i n}=40 \mathrm{~mm}$ and $4.7 \mathrm{~dB}$ for $d_{i n}=50 \mathrm{~mm}$ is obtained, being the received power higher when the large antenna is used.

When the spacing between antennas is higher than $50 \mathrm{~mm}$, the behaviour of the received power varies depending on the inner antenna used. The received power drops considerably as the separation distance between antennas is increased when using the large antenna. It can be produced by the multipath effect as well as the efficiency of this antenna for these scenarios in comparison with the miniaturized one. Moreover, the received power is around the noise floor for frequencies above $6 \mathrm{GHz}$ using both transmitting antennas. 


\section{IB2OFF}

Figure 3.46 shows the relative received power as a function of frequency in the IB2OFF scenario. In this scenario, it can be observed that the noise level is approximately at a relative received power of $-110 \mathrm{~dB}$.

The received power is higher using the miniaturized antenna from 3.1 to 6 $\mathrm{GHz}$ for all distances. One can also observe how the power has a smoother decrease from 4 to $6 \mathrm{GHz}$ in case of using the miniaturized antenna. For both antennas, the received power does not vary significantly when $d_{o f f}$ is increased. This is explained by the fact that, in this scenario, the increments of $d_{\text {off }}$ are performed being the air the propagation, whereas the inner antenna is fixed. On the contrary, the relative received power for IB2OB scenario decreased considerably with the increments of $d_{i n}$ since, in that case, the outer antenna is fixed and the inner antenna is moved inside the phantom. Besides, it can be noted that using both antennas the relative received power is around the noise floor above $6 \mathrm{GHz}$ as well.

\section{Conclusions of the Comparison of the Two Antennas}

The results presented in the previous sections comparing the performance of both antennas evidence that the antennas have a similar behaviour in both propagation scenarios. Using either of the two antennas, the relative received power is around the noise floor above $6 \mathrm{GHz}$. The results also shows the positive impact of designing the in-body antenna considering the propagation medium from the initial designing stage. In this way, a tiny UWB antenna, which maintains a quasi-omnidirectional radiation pattern and its bandwidth, can be achieved in order to perform reliable measurement campaigns to evaluate UWB .

\subsection{UWB On-Body Antennas}

As mentioned, implantable antennas are designed to work inside the body. In the design stage, the human body tissues are considered since they change the characteristics of the antennas. In this section, new UWB on-Body antennas are assessed and discussed as antenna candidates to communicate with inbody devices. In order to achieve a large bandwidth, the direct antenna design procedure is applied. 


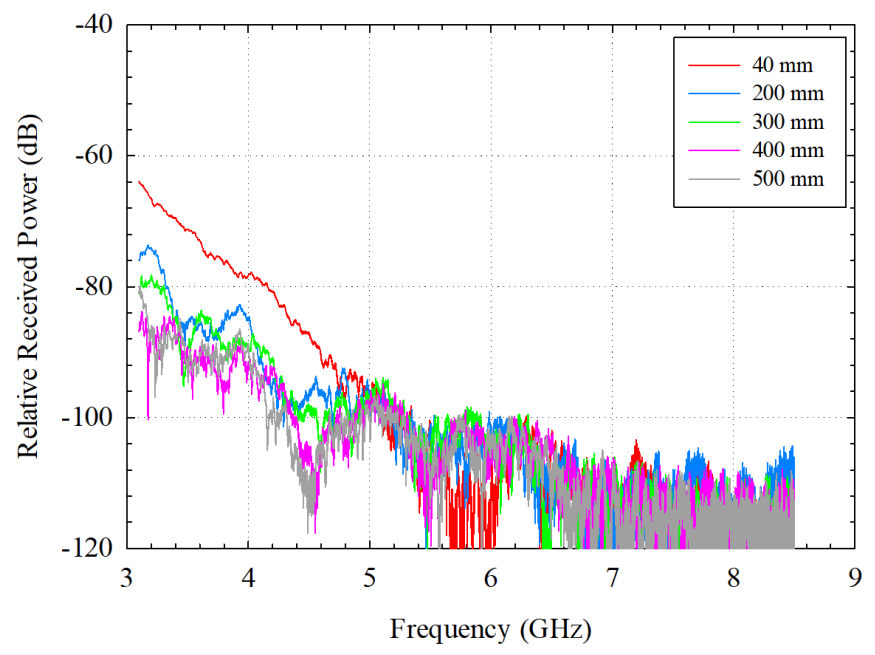

(a)

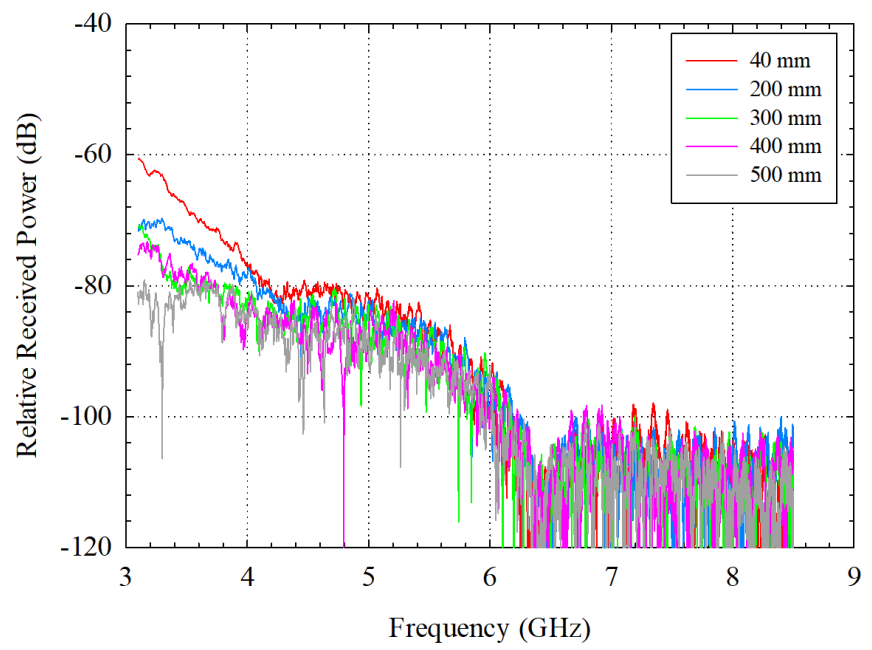

(b)

Figure 3.46: Relative received power as a function of the frequency in the IB2OFF scenario for different values of $d=d_{\text {off }}$ when (a) the large and (b) the miniaturized antennas acted as transmitters. 


\subsubsection{Simulation setup 6 Antenna Candidates}

Since there are not many UWB on-body antenna models in literature, wellknown technologies should be considered as possible candidates. Slotted patch antennas can achieve large bandwidths with low-profile structures within UWB [80], [81]. Other relevant feature of this kind of antenna is the low and compact profile of its designs as well as the versatility and adaptability of its models.

In this section, several UWB on-body slot patch antenna models are proposed and optimized by the direct antenna design procedure explained in Section 3.2.2. These antenna models have been designed and optimized by considering the most relevant human tissues of the abdominal region as proposed in [82]. The simulations consider the human body as a multilayer tissue model [83]. In particular, the multilayer model depicted in Figure 3.47 is considered. CST sotware is used to perform the antenna design.

The antenna is placed over five different tissue layers. These layers correspond with the main human body tissues involved in the transmission from the small intestine to the body surface as proposed in [82]. The antenna is considered to be in physical contact with the skin. The thickness of each layer is depicted in Figure 3.47. The dielectric properties of human body tissues, electric conductivity and relative permittivity, were provided by C. Gabriel [12]. From the Gabriel's data the dielectric features of human muscle tissue within UWB frequency range are used in CST in order to perform the simulations.

\begin{tabular}{|cc|}
\hline Small Instestine Wall & $0.5 \mathrm{~mm}$ \\
\hline Fat $_{\text {Layer2 }}$ & $15 \mathrm{~mm}$ \\
\hline Muscle & $10 \mathrm{~mm}$ \\
\hline Fat $_{\text {Layer1 }}$ & $20 \mathrm{~mm}$ \\
\hline Skin & $1.5 \mathrm{~mm}$ \\
\hline UWB Antenna \\
\hline
\end{tabular}

Figure 3.47: Multilayer Antenna Model for the CST simulations.

Two UWB on-body slotted patch antennas have been designed, miniaturized and optimized considering the requirements mentioned in Section 3.1.2.2. In both slot antenna designs, a fork-shaped microstrip feeding line structure is chosen. This is by the fact that this kind of feeding can get larger bandwidth than that obtained with a conventional one [84]. Besides, a really compact an- 
tenna structure can be achieved by using this feeding technique. In Figure 3.48, the proposed feeding structure is shown.

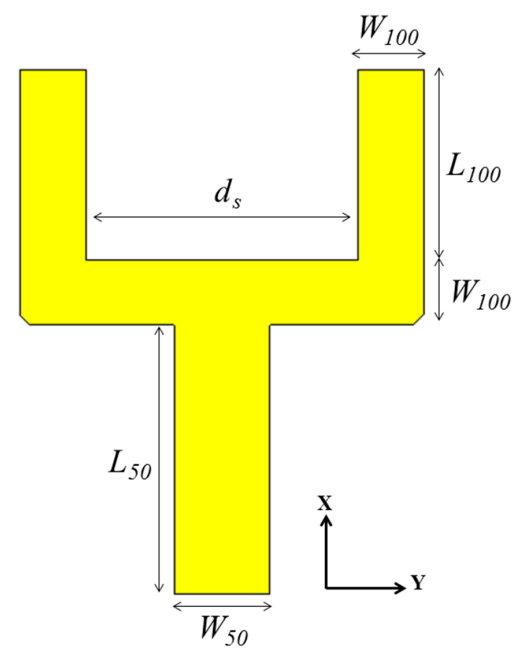

Figure 3.48: Fork-shaped microstrip feeding line.

The fork-shaped structure is based on two symmetrical $100 \Omega$ microstrip lines which are connected in parallel to the $50 \Omega$ feed line. The microstrip feeding line width $\left(\mathrm{W}_{50}\right)$, the stubs width $\left(\mathrm{W}_{100}\right)$, and the separation between the two stubs $\left(\mathrm{d}_{s}\right)$, are computed in order to achieve an input impedance of 50 $\Omega$. Otherwise, the microstrip length is $\mathrm{L}_{50}$ and the stubs length is $\mathrm{L}_{100}$. The dimensions of the feeding line are provided in Table 3.1.

Table 3.1: Dimensions of fork-shaped feeding line.

\begin{tabular}{|c|c|}
\hline Parameter & Value $(\mathrm{mm})$ \\
\hline $\mathrm{W}_{50}$ & 6.38 \\
\hline $\mathrm{W}_{100}$ & 4.30 \\
\hline $\mathrm{d}_{s}$ & 18.13 \\
\hline $\mathrm{L}_{50}$ & 12.67 \\
\hline $\mathrm{L}_{100}$ & 17.90 \\
\hline
\end{tabular}

The first design consists in a conventional rectangular-shaped slotted patch in free space. The antenna structure is miniaturized and optimized by considering the five-layered model described in Figure 3.47 and the direct miniaturization 

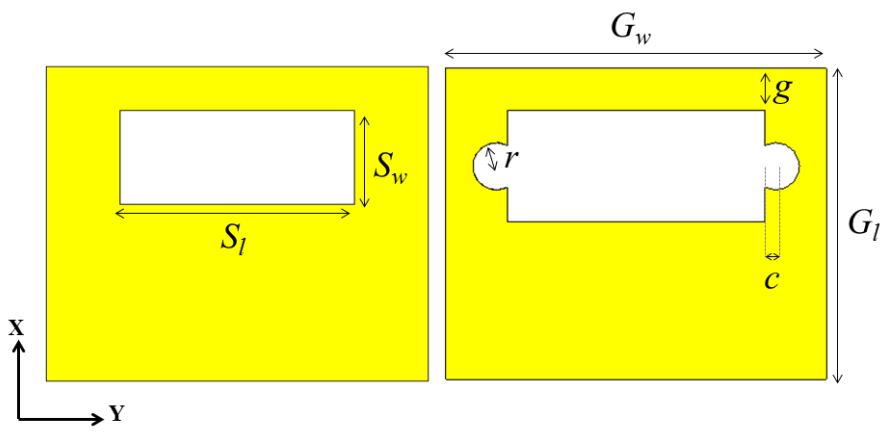

Figure 3.49: Proposed slotted patch structures.

technique explained in Section 3.2.2. In this case, the antennas are in direct contact with the tissue layer on one side instead of being completely wrapped by the tissue. The second design adds two circles at the ends of the slot to enhance the impedance matching in a wider range of frequencies. These two designs are depicted in Figure 3.49.

The ground plane dimensions are $\mathrm{G}_{l}$ and $\mathrm{G}_{w}$. The rectangular slot and the slot with circles have a width of $S_{w}$ and a length of $S_{l}$. The distance between the edge of the slot and the centre of the circle is $\mathrm{c}$, and the radius of the circle is r. Finally, the gap between the slot and the upper edge of the ground plane is g. The dimensions of the slots are summarized in Table 3.2.

Table 3.2: Dimensions of slot models.

\begin{tabular}{|c|c|c|}
\hline Parameter & Rectangular slot $(\mathrm{mm})$ & Slot with circles $(\mathrm{mm})$ \\
\hline $\mathrm{G}_{l}$ & 40.5 & 40.5 \\
\hline $\mathrm{G}_{w}$ & 49.5 & 49.5 \\
\hline $\mathrm{S}_{w}$ & 9.73 & 14.55 \\
\hline $\mathrm{S}_{l}$ & 23.56 & 33.50 \\
\hline $\mathrm{g}$ & 5.50 & 5.50 \\
\hline $\mathrm{c}$ & - & 1.41 \\
\hline $\mathrm{r}$ & - & 3 \\
\hline
\end{tabular}

In order to receive the maximum power from sensors located inside the human body, it is important to focus the radiation pattern of the designed antenna in the direction of interest. Considering the slot antenna theory, this kind of structures presents an omnidirectional radiation pattern [60]. To increase 
the directivity of the antenna reducing the backward radiation, a reflecting element is included. Figure 3.50 depicts the complete antenna structure including a reflecting plane and the dielectric substrate. On the one hand, the position and the size of the reflector are thoroughly studied in order to focus the power radiation in the desired direction. After an optimization process, a reflector length of $\mathrm{R}_{l}=50 \mathrm{~mm}$, and a distance between the feeding structure and the reflector of $\mathrm{d}_{r}=10 \mathrm{~mm}$ are chosen for this purpose. The width of the reflector, $\mathrm{R}_{w}$, is equal to that of the ground plane $\left(\mathrm{G}_{w}\right)$ in order to minimize the overall size of the antenna. Moreover, a Rogers 3003 dielectric substrate, which has a relative permittivity of 3 and a thickness of $1.524 \mathrm{~mm}$, is used.

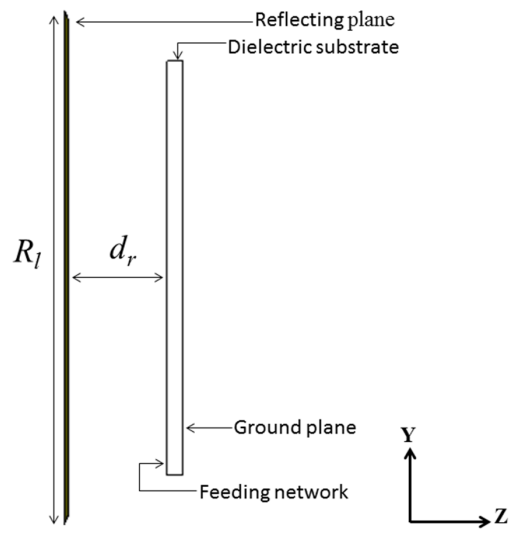

Figure 3.50: Side view of the proposed antenna structure.

\subsubsection{Simulated Results}

\subsubsection{Reflection Coefficient}

As can be observed in Figure 3.51, the reflection coefficient values obtained with the rectangular slot are below $-10 \mathrm{~dB}$ from $4.31 \mathrm{GHz}$ to $6.10 \mathrm{GHz}$, which means an absolute bandwidth of $1.79 \mathrm{GHz}$ and a relative bandwidth of 34.39 \%. Moreover, it can be noted how the bandwidth improves by adding the circles at the ends of the slot. In this case, the antenna matching covers from $3.18 \mathrm{GHz}$ to $5.52 \mathrm{GHz}$, achieving an absolute bandwidth of $2.34 \mathrm{GHz}$ at the low part of UWB, and a relative bandwidth of $53.79 \%$. Besides, a matching band is achieved from $5.9 \mathrm{GHz}$ to $7.15 \mathrm{GHz}$, obtaining an absolute bandwidth of 1.25 $\mathrm{GHz}$ and a relative bandwidth of $18.80 \%$. Thus, the optimization of the shape 


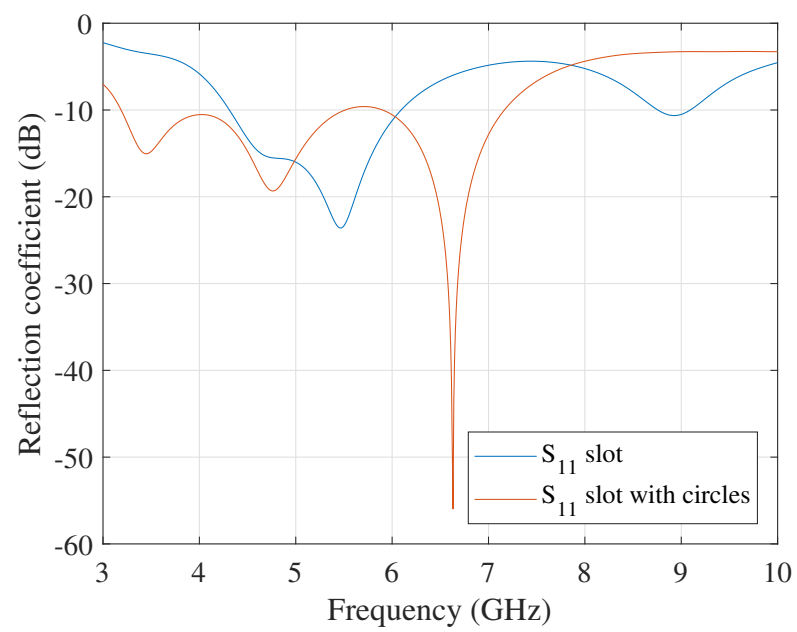

Figure 3.51: Simulated reflection coefficient for the slotted patch structures within UWB.

of the slot by adding the circles implies an improvement of the bandwidth of $550 \mathrm{MHz}$ and $19.40 \%$ in absolute and relative terms, respectively.

\subsubsection{Input Impedance}

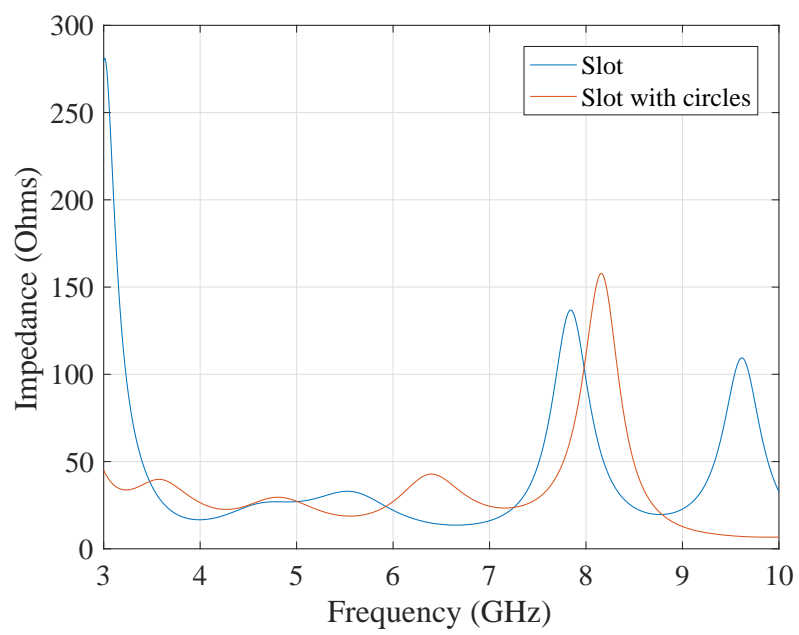

Figure 3.52: Real part of complex impedance for each slot patch structure. 


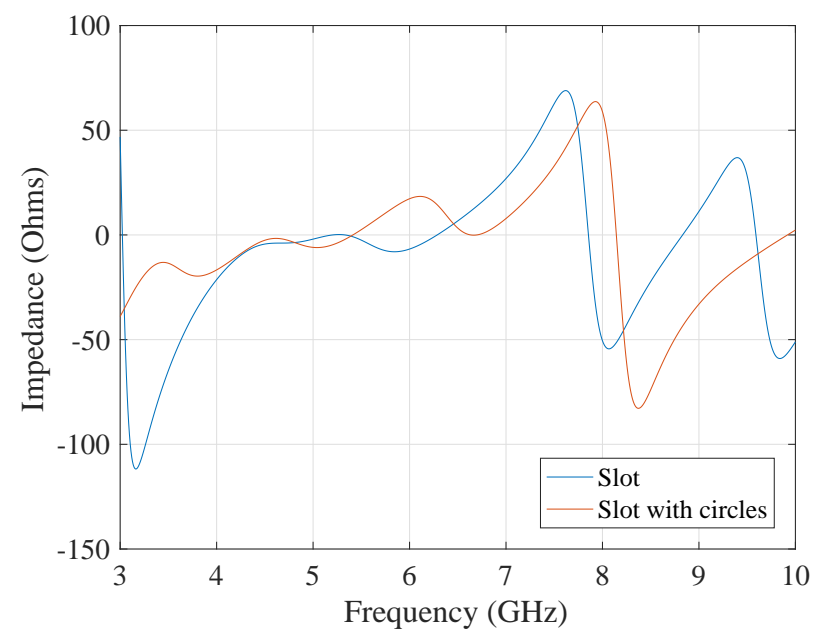

Figure 3.53: Imaginary part of complex impedance for each slot patch structure.

As can be seen from Figure 3.52, the value of the resistance is stabilized between $20 \Omega$ and $50 \Omega$ from $3.1 \mathrm{GHz}$ to $7.75 \mathrm{GHz}$ in both designs. Moreover, as can be observed in Figure 3.53, the reactance of the slot with circles is close to zero up to $7.75 \mathrm{GHz}$. On the contrary, in the case of the rectangular slot, the reactance is close to zero in a narrow frequency band. Since the performance of the slot patch antenna with circles is better, hereinafter this antenna model has only been considered.

\subsubsection{Near-field Radiation Pattern}

Considering that the implanted and the on-body antennas are close to each other and the wavelength is shorter due to the dielectric properties of tissues, it can be concluded that the antennas mainly work on the Fresnel region [60], [72]. Accordingly, Figure 3.54 shows the near-field radiation patterns of the slot with circles from $3.1 \mathrm{GHz}$ to $6.1 \mathrm{GHz}$. The width of the tissue layers match the width of the antenna in order to reduce the computational cost of the simulations [61]. 

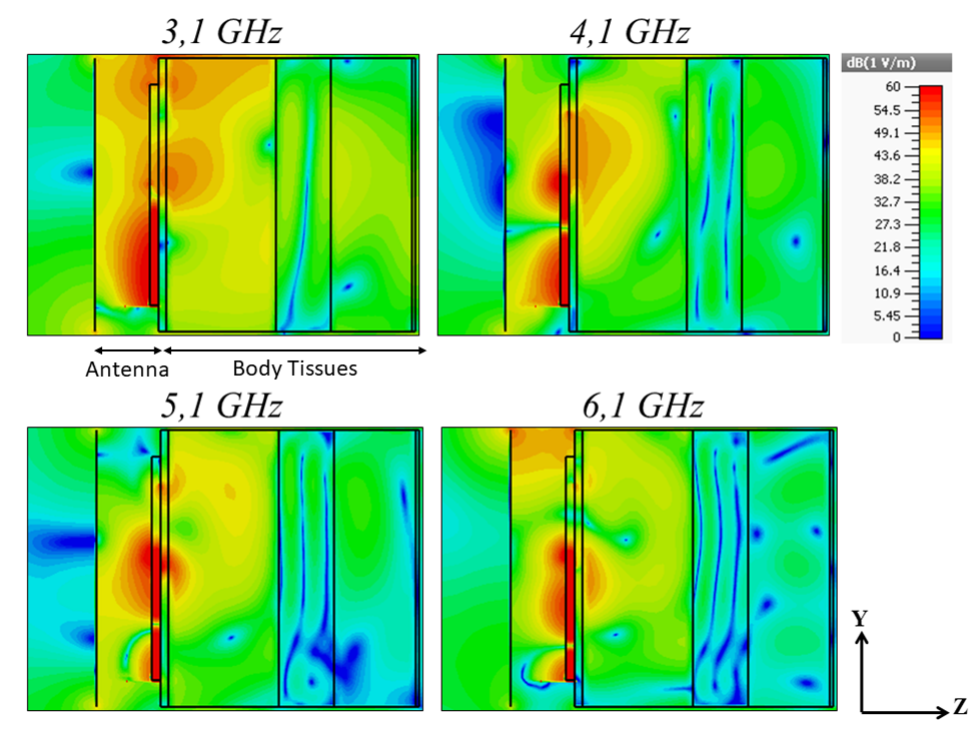

Figure 3.54: Near radiated E-Field $(\mathrm{dBV} / \mathrm{m})$ in the human tissues.

As can be seen, the slot loaded with circles achieves good results in terms of field penetration into the human tissues. However, as expected, both field penetration and near-field radiating pattern get worse as the frequency increases. This happens because the losses in the human tissues grow as the frequency increases [12]. On the other hand, the effect of the reflecting plane can be noticed. The electrical field is focused into the human tissues and the penetration through them increases. Moreover, the backward radiation is minimized as expected.

\subsubsection{Manufacturing $\mathcal{G}^{3}$ Testing}

A prototype of the slot loaded with circles is fabricated at the iTEAM facilities. In Figure 3.55, the manufactured model of this design is shown. In order to hold the reflector plane and increase the consistency of the whole structure, four nylon screws are included during the manufacturing process.

After the manufacturing process, the antenna performance is checked in order to verify the accuracy of the simulation setup considered in the design stage. Hence, $\mathrm{S}_{11}$ is measured over the belly of a human subject. The antenna is placed over the human skin surface as can be seen in Figure 3.56. The measurement setup is shown on the left-hand side of Figure 3.56. In order 

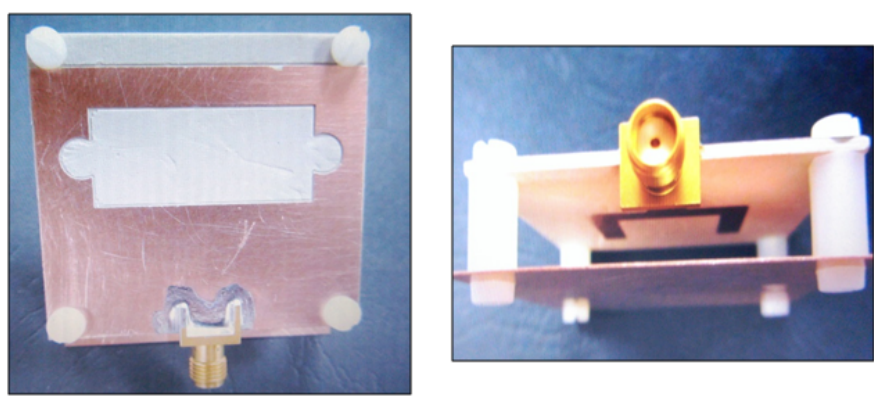

Figure 3.55: Manufactured prototype of the slot patch antenna with circles.

to measure the reflection coefficient, a Keysight N5227A VNA is used. The VNA is calibrated through a full port calibration. To protect the antenna, and avoid direct contact with the human skin, it is covered with a plastic bag in the measurement process. On the one hand, the comparison between the simulated $\mathrm{S}_{11}$ values of the slotted patch antenna with circles with those obtained on the human belly are shown in Figure 3.57. It can be observed that both results are in close agreement. The slight differences observed can be attributed to the divergences between the thickness of the tissue layers of the human subject used during the measurements and the thickness of the tissues used in the simulation process, along with the use of the plastic bag. Nevertheless, $\mathrm{S}_{11}$ values are still below $-6 \mathrm{~dB}$ between $3 \mathrm{GHz}$ and $5.3 \mathrm{GHz}$, achieving an absolute bandwidth greater than $2 \mathrm{GHz}$ within the low part of UWB.

On the other hand, the reflection coefficient of the slot patch antenna with circles is obtained by using a living porcine model at the in vivo experiment described in Section 2.3.2. The on-body antenna is placed in 13 different locations on the belly of the pig subject. The obtained values are depicted in
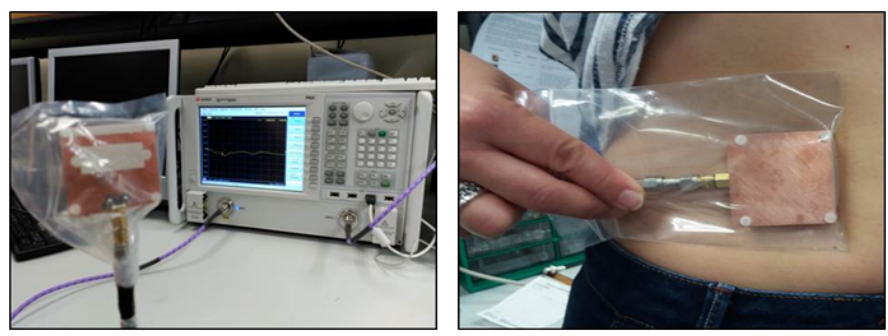

Figure 3.56: Measurement setup. 


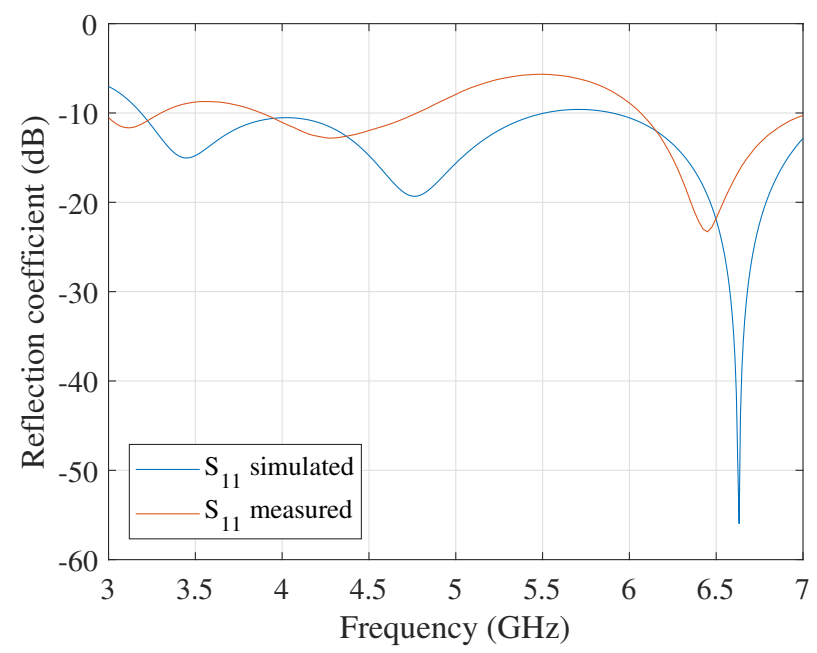

Figure 3.57: Simulated and measured reflection coefficient of the manufactured antenna.

Figure 3.58. Considering the mean values of all receivers shown in Figure 3.58, the reflection coefficient values are below $-10 \mathrm{~dB}$ from $3.3 \mathrm{GHz}$ to $4.2 \mathrm{GHz}$ and from $5.3 \mathrm{GHz}$ to $5.9 \mathrm{GHz}$, which means an absolute bandwidth of $0.9 \mathrm{GHz}$ (relative bandwidth of $10.7 \%$ ) and $0.6 \mathrm{GHz}$ (relative bandwidth of $10.7 \%$ ). It is worth mentioning that the mean values of the reflection coefficient are below $-6 \mathrm{~dB}$ within the whole frequency range under test.

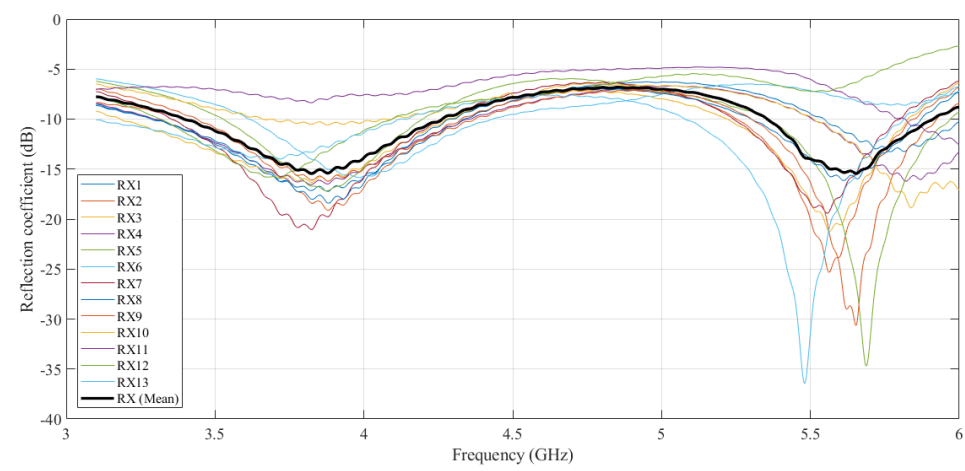

Figure 3.58: Reflection coefficient of the slotted patch antenna over the pig's belly. 


\section{Chapter 4}

\section{UWB Channel Characterization}

As mentioned in Chapter 1 of this thesis, UWB technology aims at enhancing the current in-body applications. Nevertheless, the main drawback of using UWB for in-body applications is the high signal attenuation caused by the human body tissues which increases dramatically with the increment of frequency. Hence, an accurate UWB in-body channel characterization is necessary in order to consider this band as the best candidate for future networks of implantable nodes.

This chapter is devoted to the UWB in-body channel characterization. Two in-body scenarios are considered depending on the location of the receiving antenna: IB2IB and IB2OB. Phantom-based and in vivo setups described in Chapter 2 are used. Firstly, the losses in the propagation medium and the diversity of the channel are assessed by means of these novel high accurate phantom-based setups. On the other hand, the results obtained from the measurements performed with these liquid chemical solutions in the IB2OB scenario are compared with the in vivo measurements. In this way, the reliability of the phantom-based measurements are tested. 


\subsection{Introduction}

\subsubsection{Promising In-Body Scenarios at UWB Frequencies}

The international standard for WBANs (IEEE 802.15.6) was published and approved in 2012 [4]. In this first version, MICS band was established as the frequency band used when at least one of the nodes involved in the communication is implanted inside the human body. As mentioned in Section 1.1.2, these scenarios are reflected on the standard in $\mathrm{S}_{1}$ (implant to implant), $\mathrm{S}_{2}$ (implant to body surface) and $S_{3}$ (implant to external) scenarios. Although new mobile communication standards encourage the use of large bandwidths to improve current applications, the medical standard has not been changed since its first publication. This fact restrains the IEEE Std. 802.15.6 to allow high data rate connections, which are achieved by other telecommunication services today in force. UWB frequency band has emerges as a potential candidate for the next generation of in-body devices because it can mitigate the limitations of the current band.

A substantial improvement in technology has always led to unthinkable new applications. High data rate wireless connections could make possible, for example, to send high definition images to improve medical diagnosis. An implant-to-implant communication scenario could be the intermediate step between an implanted network and a network outside the body. Internal nodes would send the collected information among them and this information would be sent to an external network using an intermediate node. The need of an intermediate step is because the main drawback of in-body communications is the high attenuation of human tissues at high frequencies [12]. Even though some studies address in-body to in-body studies where all the nodes are implanted [41], the literature is lack of measurements for IB2IB configurations within UWB frequency band.

Most popular propagation scenario in WBAN networks is the IB2OB scenario in which wireless communications are established between implanted sensors inside the body and on-body antennas located over the skin surface. This is the typical scenario of consolidated medical systems such as the capsule endoscopy. As in the IB2IB case, a quantitative enhancement in these systems would lead to countless new improvements in the wireless medical field. Most popular UWB schemes are the pulse-based schemes, which use large bandwidths, and multicarrier-based schemes, which split the available spectrum in subbands. UWB impulse radio (UWB-IR) and multiband orthogonal frequency-division multiplexing (MB-OFDM) are examples of these 
transmission schemes, respectively. The use of this UWB transmission schemes can considerably improve the performance of current medical devices such as the capsule endoscopy.

\subsubsection{Path Loss Models}

The main radio channel feature reported in the literature is the path loss and its fitting model. In this thesis, the attenuation of RF signal between the input terminals of the transmitter and the output terminals of the receiver is analyzed. ITU-R defines this attenuation as system loss. However, we use the term path loss since it is commonly used in the related literature. Thus, the comparison with other works can be performed easily.

As mentioned in Chapter 3, the VNA measures the forward transmission coefficient $\mathrm{S}_{21}$, which is the frequency transfer function $(H(f))$.

The path loss values can be computed from the $H(f)$ as [10]:

$$
P L(d B)=-10 \log _{10}\left(\overline{|H(f)|^{2}}\right),
$$

with $H(f)$ being the frequency transfer function in $\mathrm{N}$ resolution points computed as $H(f)=\left|S_{21}\right| e^{-j \angle S_{21}}$ where $\left|S_{21}\right|$ and $\angle S_{21}$ are module and phase in radians of the $S_{21}$, respectively.

According to Equation (4.1), the path loss models found in the literature can be classified depending by their dependence on distance as:

- Linear, in which the path loss in decibels varies linearly (slope $\alpha$ ) with the distance between antennas, $d$, as: $P L(d B)=C+\alpha d$, where $C$ is a constant.

- Power, where the path loss in decibels varies with the power (exponent $\gamma$ ) of the distance between antennas as: $P L(d B)=C+\alpha d^{\gamma}$.

- Log-distance, in which the path loss in decibels is linearly dependent (slope $n$ ) with the logarithm of the distance between antennas as: $P L(d B)=$ $C+10 n \log _{10} d$.

Sometimes, these models also include a shadowing term that statistically models -typically with a Gaussian function- the dispersion of the samples around the fitting model. 


\subsubsection{Correlation}

Channel diversity of UWB systems can enhance the channel performance and thus enable new applications. UWB systems can achieve highly accurate localization algorithms [85]. These techniques can be influenced by the correlation among transmitters and receivers [86]. Hence, future algorithms for the localization of implanted sensors, such as the capsule endoscope, may be affected by the channel diversity in both transmission and reception [41].

The diversity of the channel in transmission and reception is assessed by computing the correlation coefficients. These coefficients are calculated as the maximum correlation between two different channel impulse responses as follows:

$$
\rho_{d_{1}, d_{2}}=\frac{E\left[h_{d_{1}}(\tau) \cdot h_{d_{2}}^{*}(\tau)\right]}{\sqrt{E\left[\left|h_{d_{1}}(\tau)\right|^{2}\right] \cdot E\left[\left|h_{d_{2}}(\tau)\right|^{2}\right]}},
$$

being $E[]$ the expected value; $h_{d_{1}}(\tau)$ and $h_{d_{2}}(\tau)$ the channel impulse responses for a distance between antennas $d_{i}$. The channel impulse responses are obtained from the Inverse Fast Fourier Transform (IFFT) of the frequency transfer function $H(f)$ within $N$ resolution points.

\subsection{In-Body to In-Body Scenario}

\subsubsection{Preliminary Analysis}

As concluded in Section 3.2.2.5, the received power reaches the noise level from a frequency value of $6 \mathrm{GHz}$. In this preliminary analysis, the bandwidth at which the received power values are above the noise will be determined.

\subsubsection{Methodology}

The measurement setup and the methodology used in this preliminary study are explained in Chapter 2, Section 2.2.2.4. For this first approach, two identical miniaturized antennas described in Section 3.2.2 are chosen. Figure 4.1 shows the manufactured antennas.

According to Figure 2.10, the antenna located on the left hand side of Figure 4.1 acts as the in-body transmitter, whereas the other one acts as the in-body receiver. The receiving antenna is placed in the middle of the $\mathrm{Y}$ axis, at $8 \mathrm{~cm}$ away from the container's wall located behind it (X axis), and at a height of $8.6 \mathrm{~cm}$ from the container's floor ( $\mathrm{Z}$ axis). The miniaturized antenna 


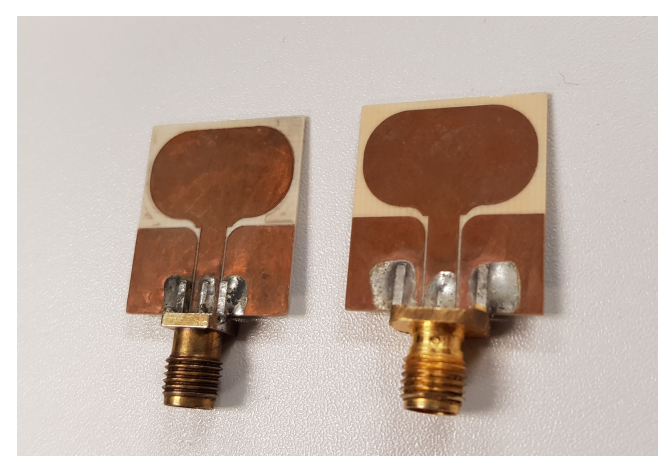

Figure 4.1: UWB in-body antennas used in the IB2IB experimental scenario

is moved in a XYZ mesh in front of the receiving antenna. The rest of the setup parameters are depicted in Table 4.1.

Table 4.1: Configuration of the VNA and the positioner. Preliminary analysis in the IB2IB scenario.

\begin{tabular}{|c|c|}
\hline Parameter & Value \\
\hline VNA: Output power & $8 \mathrm{dBm}$ \\
\hline VNA: Frequency band & $3.1-8.5 \mathrm{GHz}$ \\
\hline VNA: Resolution bandwidth & $3 \mathrm{kHz}$ \\
\hline VNA: Resolution points & 1601 \\
\hline VNA: Snapshots of the channel (Traces) & 5 \\
\hline Positioner: Step resolution & $\Delta \mathrm{x}=\Delta \mathrm{y}=\Delta \mathrm{z}=1 \mathrm{~cm}$ \\
\hline Positioner: XYZ grid mesh & $11 \times 11 \times 2$ \\
\hline
\end{tabular}

\subsubsection{Discussion}

As described in the previous section, the measurements are performed in a bandwidth ranging from 3.1 to $8.5 \mathrm{GHz}$. Figure 4.2 shows the frequency transfer function obtained from several in-body locations in this preliminary study. It can be noted that the received power significantly drops above 5.5 GHz. This is due to the fact that the losses in the muscle phantom at high frequencies grow significantly (see Figure 2.5). Therefore, the measured bandwidth should be lower than this frequency value in order to obtain non-noisy samples. In this way, the diversity of the channel and the path loss can be properly assessed. 


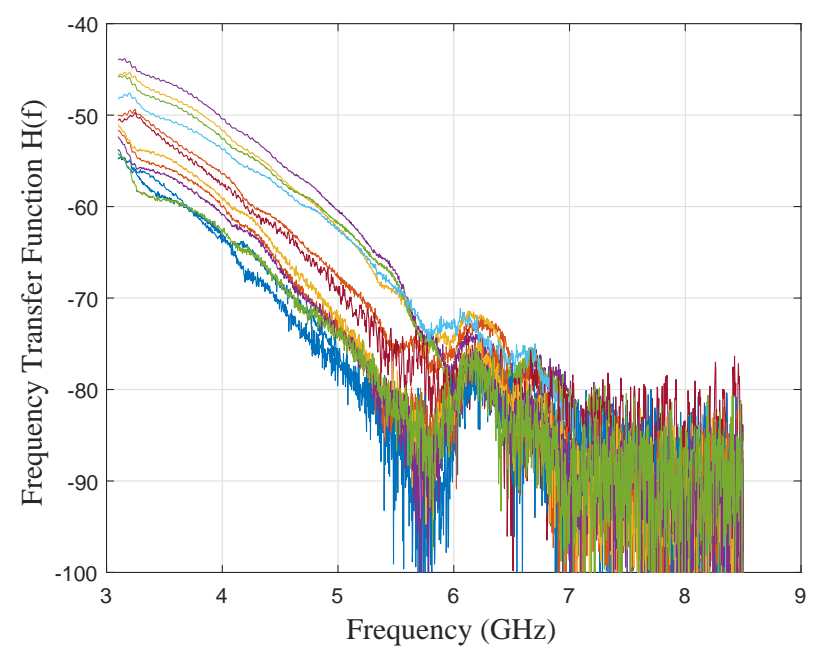

Figure 4.2: Frequency transfer function $H(f)$ in several in-body locations.

\subsubsection{In-Body to In-Body Channel Characterization}

According to the results presented in the previous section, the studied bandwidth is reduced for the IB2IB scenario in the following sections. From the obtained values, the path loss and the diversity of the channel are assessed in order to research the UWB channel performance for IB2IB communications.

\subsubsection{Methodology \&3 Data Processing}

As in the preliminary analysis, the measurement setup used is the one described in Section 2.2.2.4. The in-body antennas are also the two identical UWB monopoles used in the preliminary analysis. Furthermore, according to Figure 2.10, the antenna located on the left hand side of Figure 4.1 acts as the in-body transmitter, whereas the other one acts as the in-body receiver. The configuration of the pieces of equipment involved in this scenario is summarized in Table 4.2.

To facilitate the understanding of the measurements, reference points in each axis are established. The in-body transmitter is placed in $5 \mathrm{Z}$ planes separated steps of $\Delta \mathrm{z}$ to each other. The plane $\mathrm{Z}=0$ is the center and reference plane, whereas the other planes are located above $(-\Delta z,-2 \Delta z)$ and below $(\Delta z, 2 \Delta z)$ from $\mathrm{Z}=0$. $\mathrm{Z}$ reference plane is at a height of $8 \mathrm{~cm}$ from the container's floor. 
Table 4.2: Configuration of the VNA and the positioner. IB2IB channel characterization.

\begin{tabular}{|c|c|}
\hline Parameter & Value \\
\hline VNA: Output power & $8 \mathrm{dBm}$ \\
\hline VNA: Frequency band & $3.1-5.1 \mathrm{GHz}$ \\
\hline VNA: Resolution bandwidth & $3 \mathrm{kHz}$ \\
\hline VNA: Resolution points & 1601 \\
\hline VNA: Snapshots of the channel (Traces) & 5 \\
\hline Positioner: Step resolution & $\Delta \mathrm{x}=\Delta \mathrm{y}=\Delta \mathrm{z}=1 \mathrm{~cm}$ \\
\hline Positioner: XYZ grid mesh & $6 \times 7 \times 5$ \\
\hline
\end{tabular}

$\mathrm{X}=0$ is at $15 \mathrm{~cm}$ from the container's walls and the transmitting antenna is moved backwards from this position. Finally, $\mathrm{Y}=0$ is in the middle of the wall of the box and the transmitter is moved to the left and to the right from this point.

As mentioned, the studied bandwidth should be reduced since the losses in the propagation medium increases dramatically above $5.5 \mathrm{GHz}$ as concluded in Section 4.2.1.1. Accordingly, the VNA is calibrated through a full 2-port calibration within the first $2 \mathrm{GHz}$ of UWB frequency range, i.e., from 3.1 to $5.1 \mathrm{GHz}$. The noise floor is at $-90 \mathrm{~dB}$.

The return losses of both antennas are previously checked to ensure the antenna matching during the measurements (see Figure 4.3). The values between antennas are slightly different due to the manufacturing process.

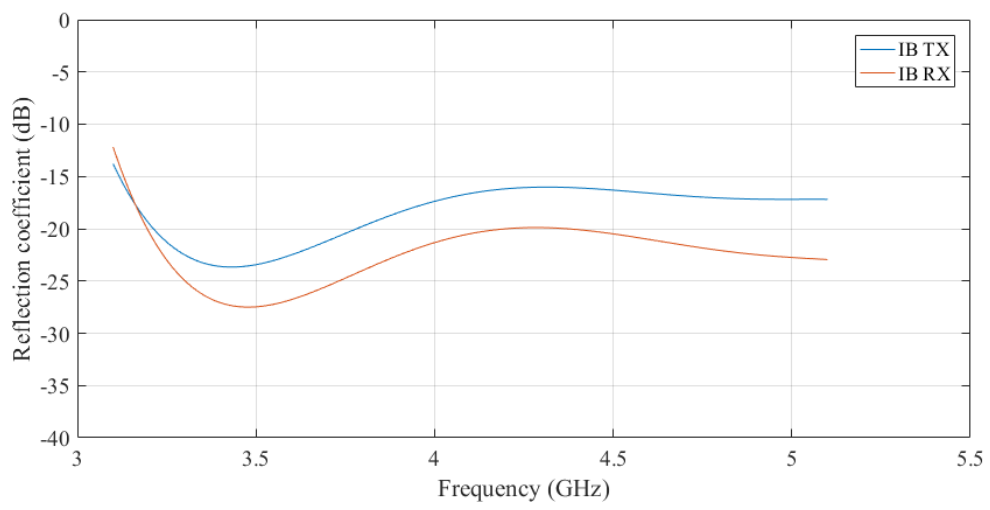

Figure 4.3: Measured reflection coefficients from 3.1 to $5.1 \mathrm{GHz}$ where both antennas are submerged into the liquid phantom. 


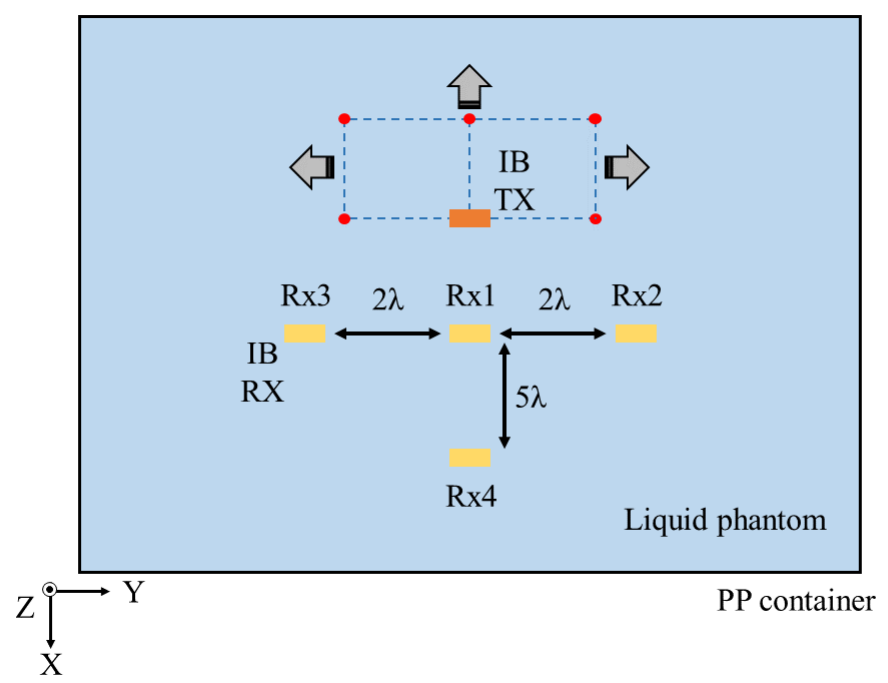

Figure 4.4: IB2IB experimental measurement setup. Locations of receiving antenna.

The XYZ mesh of $6 \times 7 \times 5$ measurement points is measured in four different locations of the in-body receiver inside the phantom (see Figure 4.4). These locations of the receiving antenna are named as RX1, RX2, RX3 and RX4. $\mathrm{RX} 1$ is at $\mathrm{Z}=0, \mathrm{Y}=0$ and $10 \mathrm{~cm}$ from the container's wall located behind it (X axis). The rest of the receiver locations are at multiples of the wavelength $(\lambda)$ from RX1 within the same height ( $Z$ plane). The value of $\lambda$ corresponds to the wavelength at the central frequency of the bandwidth under analysis (4.1 $\mathrm{GHz}$ ) considering the propagation speed inside the phantom. This wavelength is 7 times shorter than the wavelength in free at the same frequency. Regarding data processing, on the one hand, the path loss values are obtained as described in Equation (4.1). Only those $S_{21}$ samples $10 \mathrm{~dB}$ above the noise level are considered to compute the path loss values in order to capture mainly the direct path contribution. On the other hand, the diversity of the channel in transmission and reception is assessed by computing the correlation coefficients as Equation (4.2). 


\subsubsection{Path Loss}

For this section, the in-body receiver is at RX1 (see Figure 4.4). RX1 is chosen because it is in the middle and thus a greater number of samples above the noise level can be obtained. Figure 4.5 depicts, on the one hand, the path loss for different heights, i.e., Z planes (see Figure 4.5(a)) and, on the other hand, the path loss considering all the values involved in (see Figure 4.5(b)). The distance between antennas ranged from 4.8 to $9 \mathrm{~cm}$. As can be observed, the linear approximation model achieves a well fit in all cases. The linear model for different heights or all of them can be expressed as follows:

$$
P L(d B)=P L_{0, z}(d B)+\alpha_{z}(d B / c m) \cdot d(c m)
$$

where $P L_{0, z}$ is the value of the path loss for either different $\mathrm{Z}$ planes or all of them when the distance between antennas in $d B, d$, tends to 0 ; and $\alpha_{z}$ is a fitting parameter in $d B / \mathrm{cm}$.

Table 4.3 presents the path loss fit parameters considering different heights as well as all the measured values. As can be observed from this table, the values are quite similar. $P L_{0, z}$ varies from 34 to $46 \mathrm{~dB}$ and $\alpha_{z}$ from 3 to 5 , approximately. On the other hand, it can be noted that those furthest planes from $\mathrm{Z}=0$ have more losses than the rest. This is because the antenna misalignment increases at the extreme planes. Moreover, the values at $-2 \Delta z$ and $2 \Delta z$ differ due to a misalignment in height between the receivers and the grid of measurement points. On the other hand, the losses are lower when the antennas are at the same height $(\mathrm{Z}=0)$.

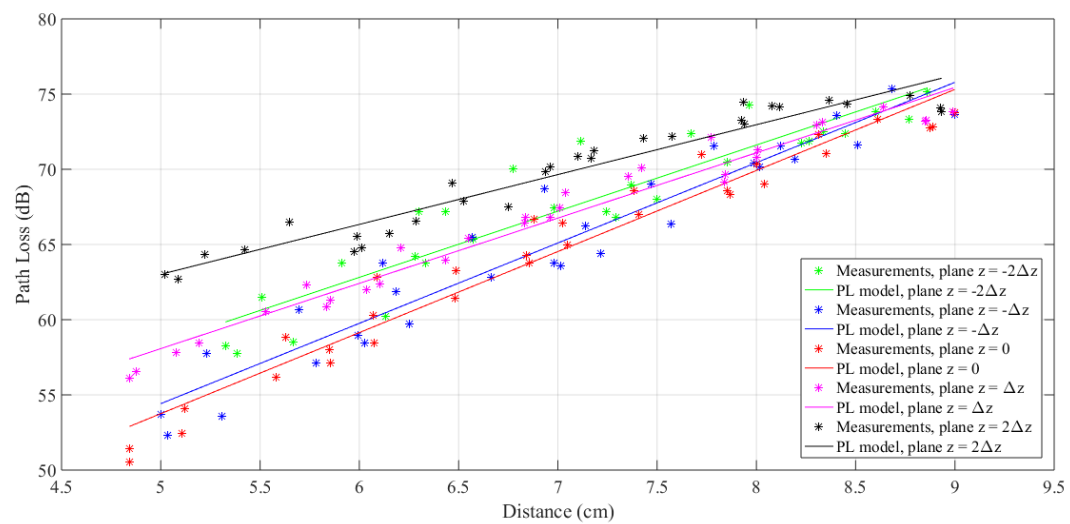

(a) 


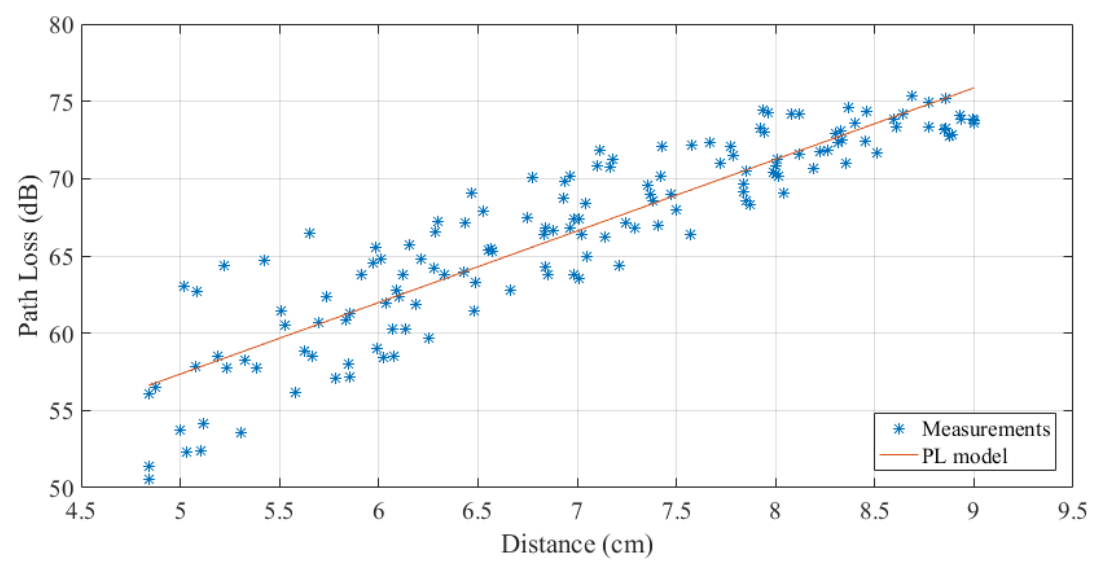

(b)

Figure 4.5: Path loss as a function of distance between antennas considering RX1. Values and models for different heights (a) and all the measured points (b).

Table 4.3: Fitting parameters of the approximation model for the IB2IB scenario.

\begin{tabular}{ccc}
\hline \hline Z plane & $\mathbf{P L}_{0, z}(d B)$ & $\alpha_{z}(d B / c m)$ \\
\hline$-2 \Delta_{z}$ & 36.4233 & 4.3987 \\
\hline$-\Delta_{z}$ & 27.7087 & 5.3416 \\
\hline 0 & 26.8048 & 5.3894 \\
\hline$\Delta_{z}$ & 36.3740 & 4.3404 \\
\hline $2 \Delta_{z}$ & 46.4496 & 3.3141 \\
\hline All & 34.2116 & 4.6290 \\
\hline \hline
\end{tabular}

When all the measured points are considered, the slope of the approximation model is an intermediate value of the above cases as expected. 


\subsubsection{Channel Correlation Modeling}

\section{Correlation in Transmission}

The diversity of the channel is evaluated by means of the correlation coefficients as Equation (4.2). In Figure 4.6, the scheme for computing the correlation coefficients in transmission is shown. The receiving antenna is in the $\mathrm{RX} 1$ position. In each $\mathrm{Z}$ plane, a reference location of the transmitting antenna is established. These reference locations are at $\mathrm{X}=0, \mathrm{Y}=0$ in each $\mathrm{Z}$ plane. Accordingly, the channel impulse response in the reference location $\left(h_{d_{1}}(\tau)\right)$ is correlated with those impulse responses located at the same $\mathrm{Z}$ plane $\left(h_{d_{2}}(\tau)\right)$.

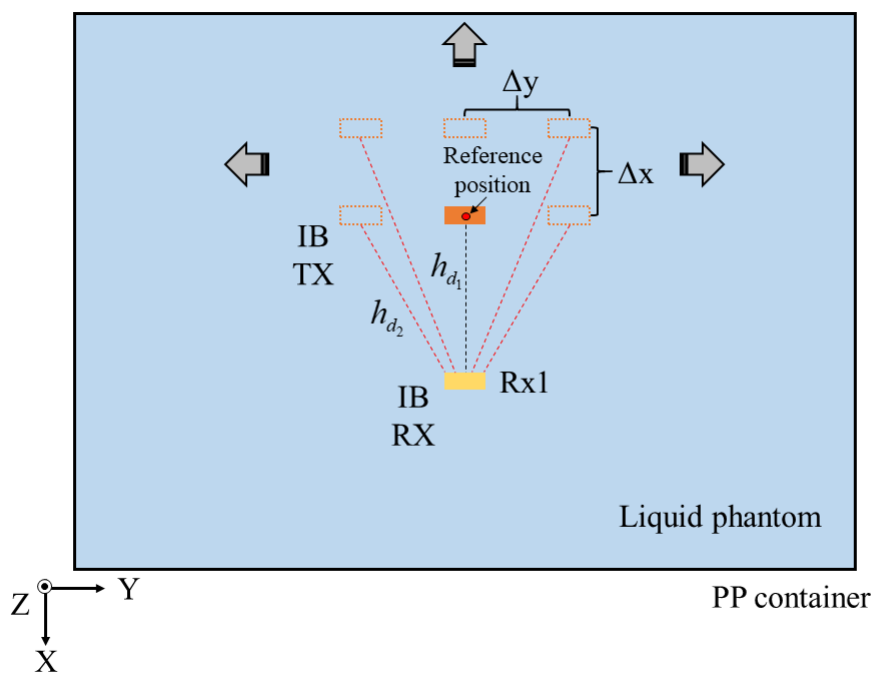

Figure 4.6: Scheme for the assessment of the correlation in transmission in the IB2IB scenario

Figure 4.7 depicts the correlation in transmission for transmitting antennas located at the same height particularized for RX1 receiver location. From Figure 4.7, one can observe that the behavior of the correlation is quite similar regardless of the $\mathrm{Z}$ plane. Besides, the correlation varies with the $\mathrm{X}$ axis but not with the $\mathrm{Y}$ axis. Moreover, the correlation drops below 0.5 when the transmitter location is above $4 \Delta x(4 \mathrm{~cm})$ from the reference transmitting antenna location. 


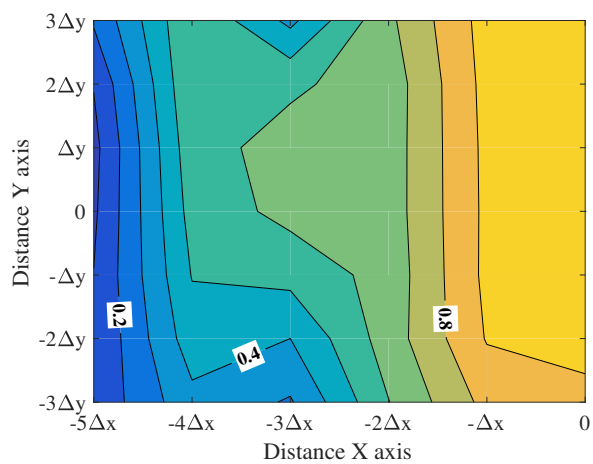

(a)

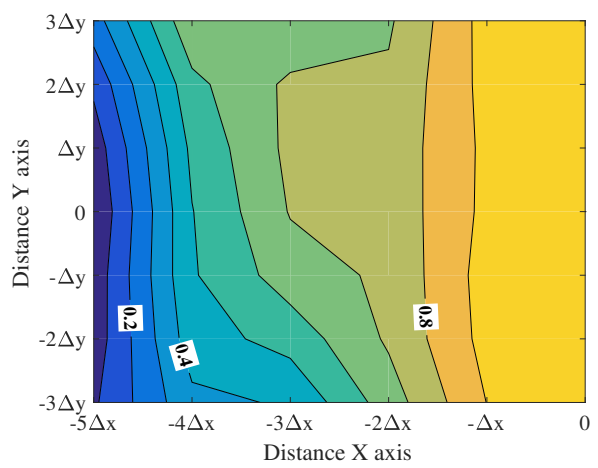

(c)

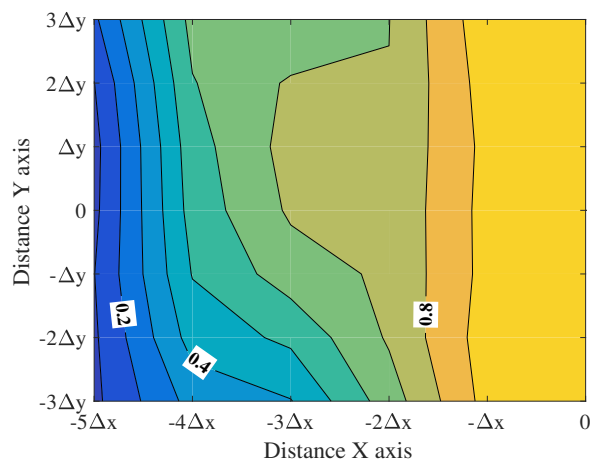

(b)

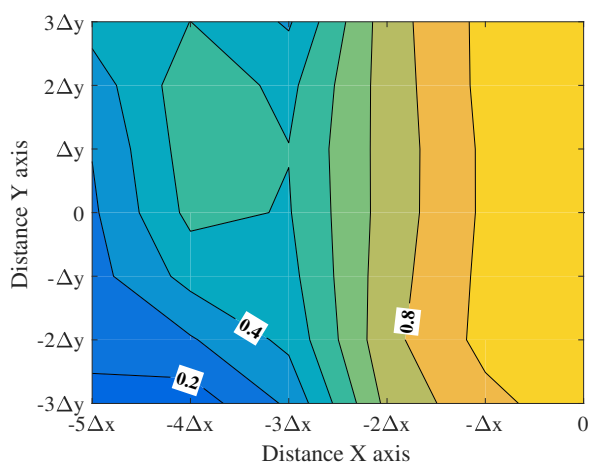

(d)

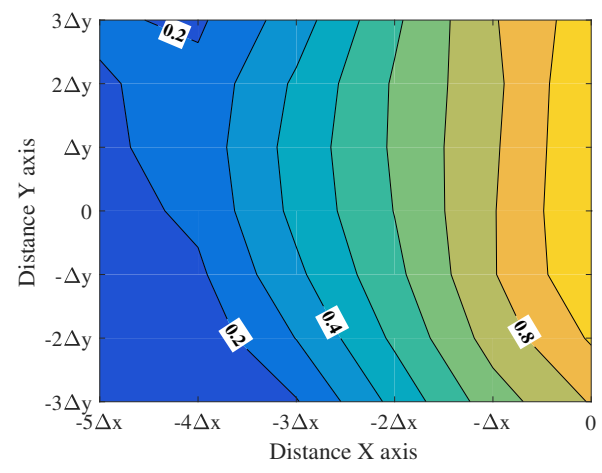

(e)

Figure 4.7: Correlation coefficients at different heights in the experimental IB2IB scenario using RX1. $\mathrm{Z}=-2 \Delta \mathrm{z}(\mathrm{a}), \mathrm{Z}=-\Delta \mathrm{z}$ (b) and $\mathrm{Z}=0$ (c) $\mathrm{Z}=-\Delta \mathrm{z}$ (d) and $\mathrm{Z}=2 \Delta \mathrm{z}$ (e). 


\section{Correlation in Reception}

In order to assess the correlation in reception, the position of different receiving antenna locations are considered. The channel impulse responses in each transmitting antenna location for all the measurement points obtained from RX2 is correlated with those obtained from the rest of the receivers, RX1, RX3, RX4 (see Figure 4.4) at the same transmitting antenna location. According to Equation (4.2), $h_{d_{1}}(\tau)$ is the channel impulse response obtained from RX2 in a transmitting antenna location, whereas $h_{d_{2}}(\tau)$ is the impulse response in the same transmitting antenna location obtained from the other receivers, i.e., $d_{1}=d_{2}$. RX1 and RX3 are $2 \lambda$ and $4 \lambda$ away from RX2 in $\mathrm{Y}$ axis, respectively, while $\mathrm{RX} 4$ is $2 \lambda$ away in $\mathrm{Y}$ axis and $5 \lambda$ in $\mathrm{X}$ axis from RX2 as well (see Figure 4.4).

Figure 4.8 shows the Complementary Cumulative Distribution Function $(\mathrm{CCDF})$ for the correlation in reception, i.e., $\mathrm{CCDF}=1-\mathrm{P}(\mathrm{X}<$ correlation coefficients $)=\mathrm{P}(\mathrm{X}>$ correlation coefficients $)$. The CCDF in each pair of receivers is computed considering all the measurement points in the whole XYZ mesh measured by each receiver. As can be observed, the correlation decreases as the distance between receivers increases. This is more significant when the receiving antennas are away in both axes (RX2\&RX4), since practically all the correlation coefficients are below 0.65. When receiver antennas are only separated in $\mathrm{Y}$ axis, the correlation increases considerably. Nevertheless, the increment of the distance between receivers in $\mathrm{Y}$ axis can reduce the correlation. As can be seen in Figure 4.8, the values of the correlation coefficients when receiving antennas are separated $4 \lambda$ (RX2\&RX3) decreases by $10 \%$ compared to the values when receivers are separated $2 \lambda$ (RX2\&RX1). However, the correlation is very high in both cases. 


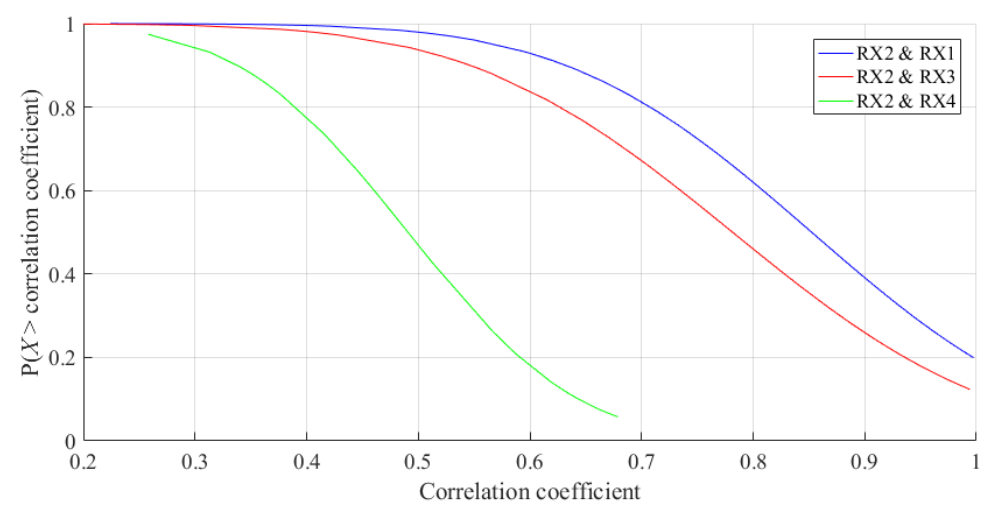

Figure 4.8: Complementary cumulative distribution function (CCDF) for correlation coefficients in reception.

\subsection{In-Body to On-Body Scenario}

As mentioned in Section 4.1.1, UWB technology can improve the features of current medical devices such as the capsule endoscopy. In order to validate this frequency band, a thorough investigation of the performance of the propagation channel at these frequencies is absolutely essential. This requires reliable measurement setups.

Throughout this section, the path loss and the diversity of the channel for UWB pulsed-based schemes and multicarrier-based are assessed and discussed in different IB2OB scenarios. The radio channel performance for the whole used bandwidth as well as for 500- $\mathrm{MHz}$ subbands is studied from an experimental point of view. On the other hand, the impact of the chosen receiving antenna on the UWB channel performance is researched as well. Also, the values obtained from a phantom-based setup are compared with those obtained in an in vivo experiment. 


\subsubsection{Path Loss Models}

\subsubsection{Effect of Receiving Antenna on Channel Performance}

In order to assess the impact of the antenna features on the radio channel performance, two different antennas have been selected. The first antenna candidate is a slotted patch antenna which was optimized by using the direct antenna design procedure explained in Section 3.2.2. The other one is a UWB monopole antenna used as on-body receiver in several experimental measurement campaigns [41], [87].

For both receiving antennas previously described, the phantom-based scenario described in Figure 2.11 has been considered. Using either the slot or the monopole antenna, the CPW monopole antenna described in Section 3.2.2 is chosen as implanted transmitting antenna. The VNA and the positioner configuration is detailed in Table 4.4.

Table 4.4: Configuration of the VNA and the positioner. IB2OB channel characterization.

\begin{tabular}{|c|c|}
\hline Parameter & Value \\
\hline VNA: Output power & $8 \mathrm{dBm}$ \\
\hline VNA: Frequency band & $3.1-5.1 \mathrm{GHz}$ \\
\hline VNA: Resolution bandwidth & $3 \mathrm{kHz}$ \\
\hline VNA: Resolution points & 1601 \\
\hline VNA: Snapshots of the channel (Traces) & 5 \\
\hline Positioner: Step resolution & $\Delta \mathrm{x}=\Delta \mathrm{y}=\Delta \mathrm{z}=1 \mathrm{~cm}$ \\
\hline Positioner: XYZ grid mesh & $7 \times 7 \times 3$ \\
\hline
\end{tabular}

The plane $\mathrm{Z}=0$ is the center plane where the transmitter and the receiver are aligned in the vertical axis, whereas the other planes are located above $(\Delta \mathrm{z}$, $2 \Delta \mathrm{z}$ ) from this plane. $\mathrm{Z}=0$ is at a height of $8 \mathrm{~cm}$ from the container's floor. $\mathrm{X}=0$ is at $2 \mathrm{~cm}$ from the container's wall and the transmitting antenna is moved backwards from this position. Finally, $\mathrm{Y}=0$ is in the middle of the wall of the box and the transmitter is moved to the left and to the right from this point. The on-body receiver is fixed in the middle of the external container's wall, whereas the in-body transmitter is moved inside the liquid phantom. Figure 4.9 shows the IB2OB measurement setup used for this measurement campaign. 


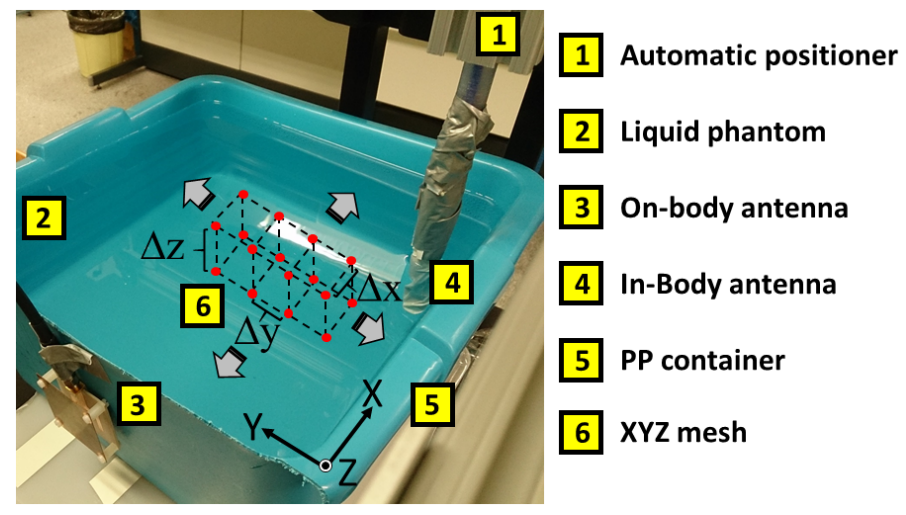

Figure 4.9: IB2OB measurement setup. Grid of measurement points.

As mentioned, the radio channel performance is assessed for separated UWB subbands as well as for the entire used bandwidth. Depending on the used receiving antenna, the noise floor is different [88]. In particular, the noise floor is at -80 and $-100 \mathrm{~dB}$ when the receiving antenna is the monopole and the slot, respectively. It is worth mentioning that only those $S_{21}$ samples $10 \mathrm{~dB}$ above the noise level are considered to capture mainly the direct path contribution whatever the receiving antenna is.

The path loss in each in-body antenna location is calculated as described in Equation (4.1). Figure 4.10 shows the path loss samples computed from $d=$ 2.5 to $7 \mathrm{~cm}$ by using both receiving antennas. According to the observed values, the linear and the log-distance model should be compared to find out the best option. On the one hand, the linear model follows the expression described in Equation (4.3). On the other hand, the log-distance model follows the following expression:

$$
P L(d B)=P L_{0, d_{r e f}}+10 \gamma \log _{10}\left(\frac{d(\mathrm{~cm})}{d_{r e f}}\right),
$$

being $P L_{0, d_{r e f}}$ the path loss value in a reference distance $d_{r e f}=1 \mathrm{~cm}$ in $d B$, and $\gamma$ the path loss exponent.

As can be observed in Figure 4.10, the path loss values vary depending on the used receiving antenna. Besides, this variation is higher as the subband is located at higher frequency bands as expected. This may be explained due to the fact that the noise floor values change depending on the on-body receiving 
antenna used. Accordingly, the maximum value of the path loss is higher for the slot antenna. Considering the whole frequency range, the path loss values obtained from both antennas are quite similar (see Figure 4.10). The trend of both models are practically the same as well. Nonetheless, the path loss values are higher for the slot antenna. This is by the fact that the slot antenna focuses the power in one direction, minimizing the received contributions from the rest. In this way, the relative received power is lower and, consequently, the path loss becomes higher.

Even though the trend of the path loss models are quite similar by using either antenna, the performance of the antenna mainly affects on the values at high frequencies. Hence, it could be more accurate to say that this a radio link budget evaluation or system loss instead of a conventional path loss model. As mentioned in the introduction of this chapter, path loss term has been considered since it is the most used in the related works in literature.

Table 4.5: Fitting parameters of the approximation models. Monopole antenna.

\begin{tabular}{|c|c|c|c||c|c|c|}
\cline { 2 - 7 } \multicolumn{1}{c|}{} & \multicolumn{6}{c|}{ Monopole Antenna } \\
\cline { 2 - 7 } \multicolumn{1}{c|}{} & \multicolumn{3}{c|}{ Linear } & \multicolumn{3}{c|}{ Log-distance } \\
\hline Frequency & $\mathrm{PL}_{0}$ & $\alpha$ & RMSE & $\mathrm{PL}_{0}$ & $\gamma$ & RMSE \\
\hline $3.1-3.6$ & 20.316 & 5.362 & 3.7009 & 6.164 & 5.974 & 3.482 \\
\hline $3.6-4.1$ & 22.161 & 5.723 & 3.542 & 7.243 & 6.350 & 3.337 \\
\hline $4.1-4.6$ & 25.278 & 5.932 & 5.0202 & 9.96 & 6.561 & 4.868 \\
\hline $4.6-5.1$ & 34.912 & 4.172 & 6.3555 & 24.085 & 4.622 & 6.302 \\
\hline $3.1-5.1$ & 23.607 & 5.316 & 3.6158 & 9.639 & 5.914 & 3.413 \\
\hline
\end{tabular}

Table 4.6: Fitting parameters of the approximation models. Slotted patch antenna.

\begin{tabular}{|c|c|c|c||c|c|c|}
\cline { 2 - 7 } \multicolumn{1}{c|}{} & \multicolumn{5}{c|}{ Slot Antenna } \\
\cline { 2 - 7 } \multicolumn{1}{c|}{} & \multicolumn{3}{c|}{ Linear } & \multicolumn{3}{c|}{ Log-distance } \\
\hline Frequency & $\mathrm{PL}_{0}$ & $\alpha$ & RMSE & PL $_{0}$ & $\gamma$ & RMSE \\
\hline $3.1-3.6$ & 14.826 & 7.405 & 5.287 & -12.202 & 9.329 & 5.345 \\
\hline $3.6-4.1$ & 26.299 & 6.627 & 5.618 & 1.482 & 8.431 & 5.531 \\
\hline $4.1-4.6$ & 36.496 & 6.394 & 5.723 & 12.432 & 8.15 & 5.619 \\
\hline $4.6-5.1$ & 53.434 & 4.502 & 5.215 & 35.829 & 5.82 & 5.004 \\
\hline $3.1-5.1$ & 22.406 & 6.846 & 4.843 & 2.814 & 8.656 & 4.84 \\
\hline
\end{tabular}




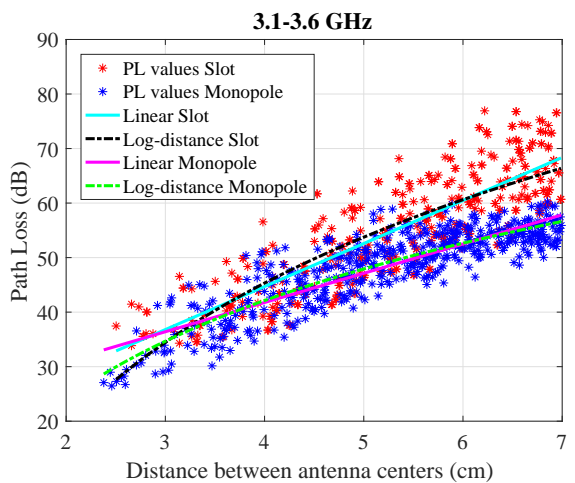

(a)

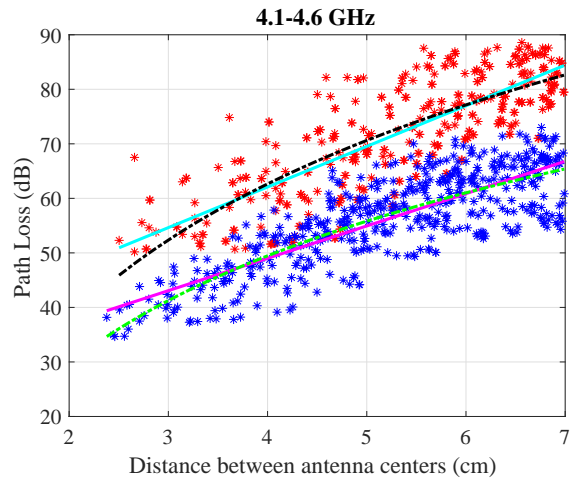

(c)

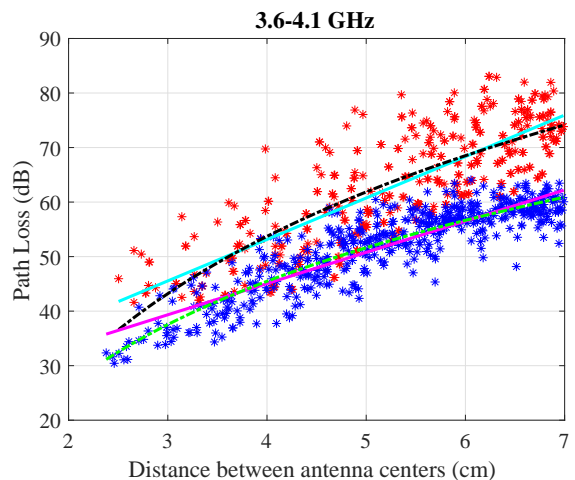

(b)

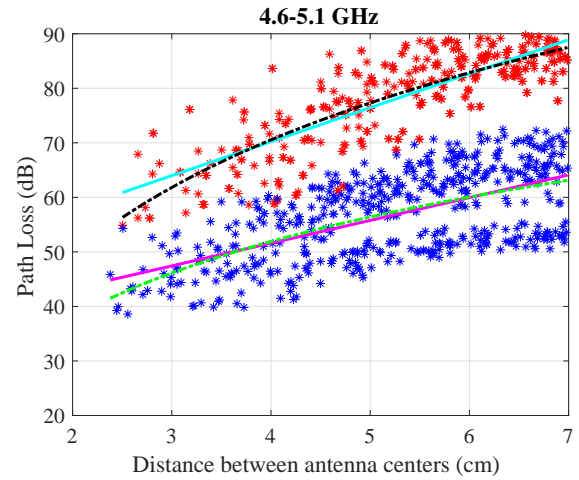

(d)

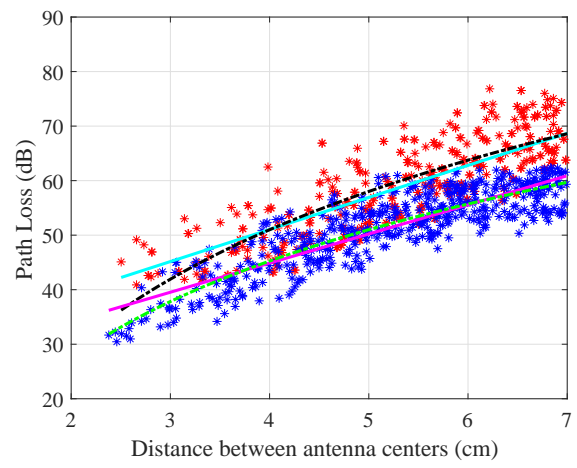

(e)

Figure 4.10: Path loss values per subband (a-d) and the whole available bandwidth (e) using two on-body receivers particularized for the IB2OB scenario. 
Table 4.5 and Table 4.6 present the path loss fitting parameters along with the RMSE for each approximation model considering each subband as well as the whole bandwidth by using both receiving antennas. As can be seen, parameter $\alpha$ is higher when the slot antenna is used, i.e., the slope of the curve is steeper. Whereas $\alpha$ is lower at higher frequency bands with the slot antenna, it keeps around five when the receiving antenna is the monopole one. The same occurs with regard to the $\gamma$ exponent in the case of the log-distance model, where the $\gamma$ value with the monopole antenna is around 6 . The differences between $\alpha$ and $\gamma$ obtained from both antennas are mainly produced by the radiation pattern. the monopole antenna takes more signals from the sides due to its omnidirectional pattern, while the patch antenna focuses power by reducing signal reception from the sides.

It is worth mentioning that the path loss exponent is lower in the upper subband for both antennas. This is by the fact that the losses reach the noise level at large distances and high frequencies, whereas they continue growing for short distances. Therefore, the slope of the model decreases.

Regarding $P L_{0}$ for linear and log-distance models, its value increases as the subband is higher in frequency for both receiving antennas. It can be observed how $P L_{0}$ is negative for the lowest frequency subband in the log-distance model for the slot antenna. This is because the $\gamma$ exponent is very high and $d_{r e f}$ is 1 , so that $P L_{0}$ is mathematically negative. On the other hand, such negative value is in concordance with a best fitting -lower RMSE- of the path loss for a linear trend. This can be observed in Table 4.6, where the RMSE is lower for the linear than for the log-distance one.

For the monopole receiver, as can be observed in Table 4.5, the best fit is achieved by the log-distance model since the RMSE is lower in all the cases. On the other hand, for the slot antenna, Table 4.6 shows that the path loss is better fit by a linear model only in the lowest frequency subband. In this case, the path loss increasingly follows a logarithmic trend as the considered frequency band is above $3.6 \mathrm{GHz}$. This can be noted since the difference between RMSE values of both models is higher as the used frequency band increases. That is, 0.087 for the second subband $(3.6-4.1 \mathrm{GHz})$ and 0.212 for the fourth one $(4.6-5.1 \mathrm{GHz})$, what leads to an increment of $143.67 \%$. In the case of the monopole antenna, the RMSE for the log-distance model is lower than the RMSE for the linear case in all the subbands studied. However, as in the case of the slot antenna, there is no big difference between linear and log-distance approximation models. 
With regard to the path loss considering the whole bandwidth, the best fitting is achieved with the log-distance model for both receiving antennas. This conclusion is in line with the results obtained in other measurement campaign where the same phantom is used [41]. On the other hand, [87] compared phantom-based and in vivo measurements in an IB2OB scenario using the phantom described in [40]. The propagation losses in [87] are higher than those obtained in this section where a high accurate muscle-like phantom is used. This is due to the fact that the dielectric values of the phantom proposed here are close to those obtained by Gabriel [12]. On the contrary, the phantom proposed in [40] overestimates the losses as concluded in [87].

\subsubsection{Experimental Models. Phantom-based vs In Vivo results}

In order to validate the results obtained from measurements performed in a laboratory facilities using phantoms, an in vivo measurement campaign is completely essential. This is the only reliable way to ensure that the laboratory tests have certain accuracy.

Observing the results obtained in the previous section, the slot antenna has better performance on than the monopole one for this purpose. Actually, this could be predictable since the slot antenna was designed and optimized to work in this propagation medium efficiently. As mentioned, at least one in vivo measurement campaign is necessary to confirm the values obtained from the phantom-based measurements. Accordingly, an IB2OB measurement campaign considering the in vivo setup explained in Section 2.3 has been carried out. For the in vivo experiment, the slot antenna acts as the on-body receiver, whereas the CPW monopole antenna described in Section 3.2.2 is used as implanted transmitter.

Due to the strict time constrains in the operating room, two different in-body points are measured with the in-body antenna implanted in two different places within the small intestine of the animal subject. It is worth mentioning that the in vivo scenario tries to emulate a real communication between an UWB device located in the small bowel, such as the capsule endoscopy, and an UWB on-body receiver located over the skin. In order to do that, the surgeon placed the in-body antenna among the guts aided by a endoscopic camera system.

Figure 4.11(a) and Figure 4.11(b) show the in-body locations through the camera system. Regarding the receiving antenna, it is located on thirteen positions over the pig belly as described in Section 2.3.2. 


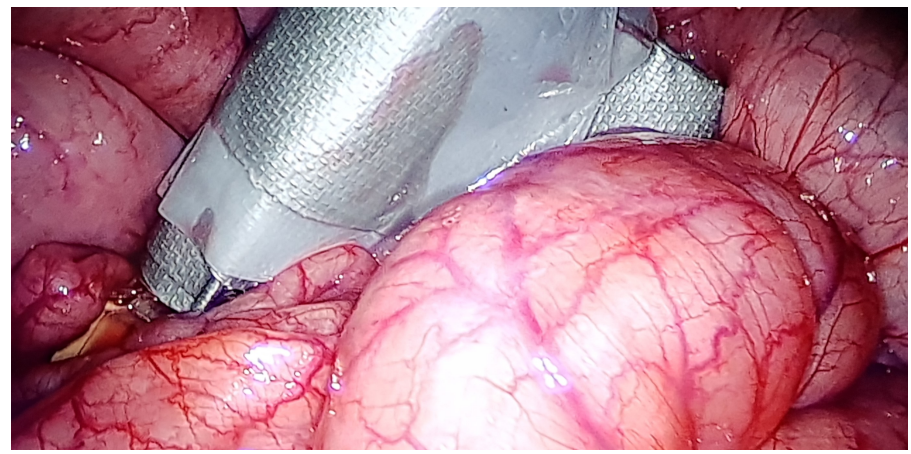

(a)

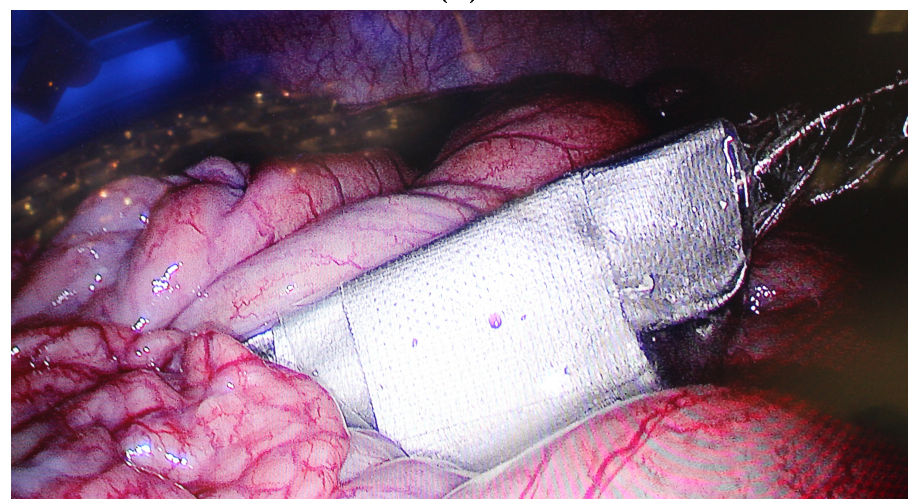

(b)

Figure 4.11: Location of the implanted antenna in the in vivo experiment

As described in Section 2.3.2.1, a specific software is designed to control and synchronize the VNA and the tracking system. The parameters entered on the VNA are exactly the same than those of the IB2OB phantom-based measurement detailed in Table 4.4. Hence, the comparison of the results obtained from both setups is simpler and more reliable. As concluded in the previous section, the antenna parameters could drastically change when a different scenario or setup is considered. In the phantom-based setup, the noise floor is at $-100 \mathrm{~dB}$ with the slot antenna as receiver. In contrast, the noise level is at $-110 \mathrm{~dB}$ when the same receiver is used in the in vivo experiment. As in the phantom-based measurements, only those $S_{21}$ samples $10 \mathrm{~dB}$ above the noise level are taken into account. 


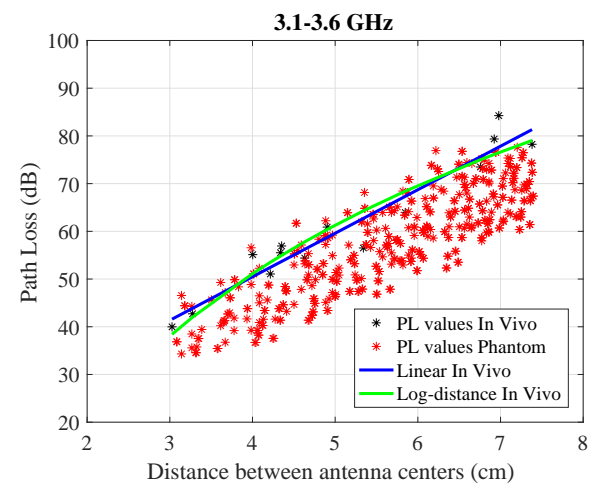

(a)

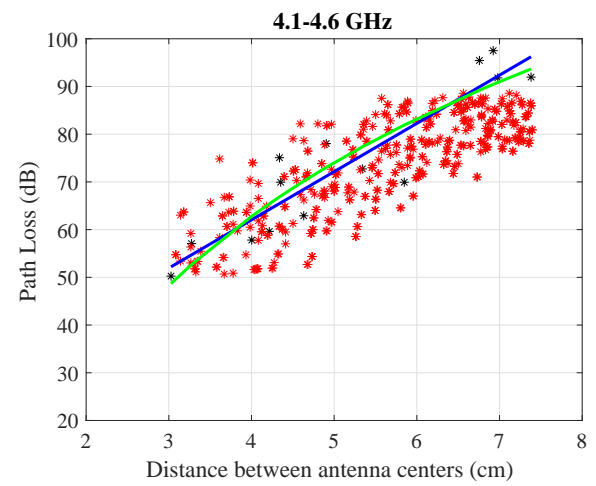

(c)

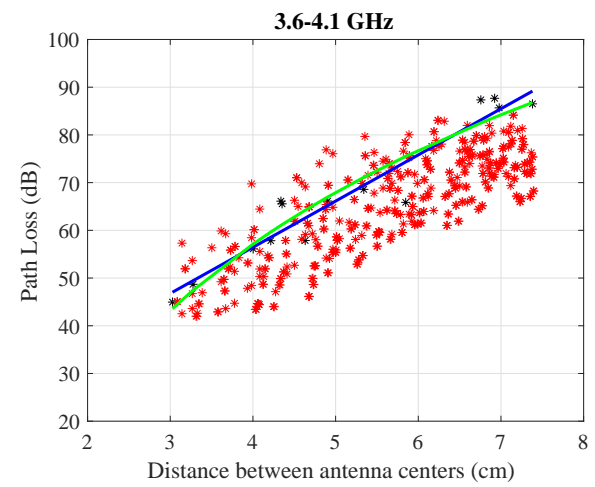

(b)

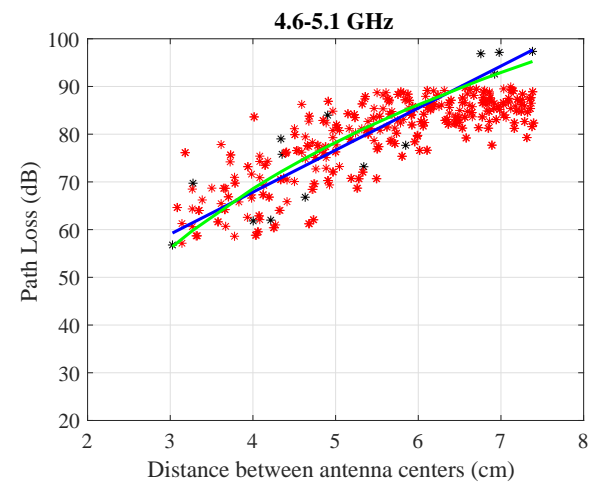

(d)

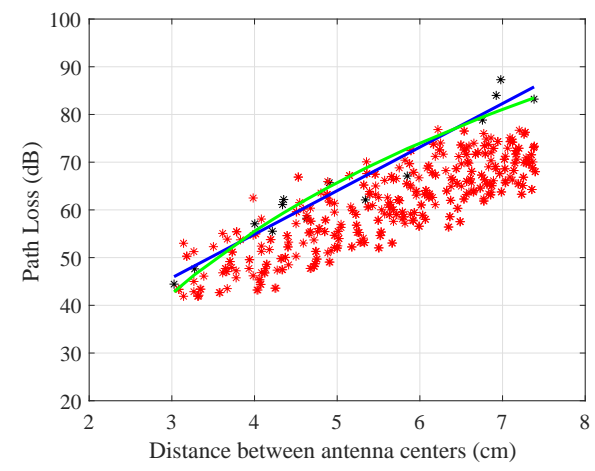

(e)

Figure 4.12: Path loss values per subband and the whole available bandwidth for in vivo (black points) and phantom-based (red points) setups particularized for the IB2OB scenario. 
Table 4.7: Fitting parameters of the approximation models for the in vivo measurements

\begin{tabular}{|c|c|c|c||c|c|c|}
\cline { 2 - 7 } \multicolumn{1}{c|}{} & \multicolumn{5}{c|}{ Slot Antenna } \\
\cline { 2 - 7 } \multicolumn{1}{c|}{} & \multicolumn{3}{c|}{ Linear } & \multicolumn{3}{c|}{ Log-distance } \\
\hline Frequency & $\mathrm{PL}_{0}$ & $\alpha$ & RMSE & $\mathrm{PL}_{0}$ & $\gamma$ & RMSE \\
\hline $3.1-3.6$ & 13.984 & 9.123 & 3.399 & -12.053 & 10.492 & 3.733 \\
\hline $3.6-4.1$ & 17.735 & 9.678 & 3.852 & -10.054 & 11.155 & 4.096 \\
\hline $4.1-4.6$ & 21.604 & 10.109 & 5.644 & -7.1765 & 11.616 & 5.926 \\
\hline $4.6-5.1$ & 32.624 & 8.808 & 5.908 & 8.254 & 10.020 & 6.345 \\
\hline $3.1-5.1$ & 17.107 & 9.425 & 3.126 & -7.868 & 10.531 & 3.613 \\
\hline
\end{tabular}

In Table 4.7, the path loss values in the in vivo experiment using the slot antenna as receiver are shown. Furthermore, the path loss values obtained from the phantom-based setup using the same receiver antenna are included in Figure 4.12 as well. The distance range obtained from the phantom-based experiment has been fitted to the minimum and maximum distance between antennas in the in vivo experiment in order to get an easier comparison. From Figure 4.12, one can observe that the path loss values obtained from the in vivo experiment are consistent with those obtained by the phantom-based setup. This is a remarkable point since these results give certain reliability to the experimental single-layer measurement setup in which the high accuracy phantom of the muscle tissue is used. In this way, the number of in vivo measurement campaigns could be decreased considerably.

When the subband increases in frequency, the path loss values of both setups are different at high distances between antennas. This is by the fact that the noise level is not the same in each case and therefore the maximum top value considered in computation. This effect can be observed at high distance and subbands where the path loss values begin to stabilize as they reach the noise level. For this reason, there are some points above in the in vivo case making it more noticeable in the higher frequency subbands. On the other hand, the arrangement of the points in the in vivo varies due to other factors such as the breathing, the blood flow and so on. In addition, it is very difficult to control the alignment of the antennas so it can produce these variations as well.

Observing Table 4.7, one can conclude that a better fit is achieved with the linear approximation model whatever the subband is. The same can be concluded in the case of the whole measured bandwidth. This can be seen in the RMSE values for the linear model which are lower than those of the log-distance one. Besides, $P L_{0}$ values are mathematically negative in almost all the cases for the log-distance model. Therefore, the linear model achieves 
the best approximation in contrast with the values obtained with phantom in which the losses tends to a log-distance model as the frequency increases. This difference in the trend of the approximation models can be explained given that the number of points measured in the in vivo experiment is limited compared to the massive measurement campaign obtained from the phantombased measurements.

With regard to the fitting parameters of the linear approximation model, $P L_{0}$ increases with frequency as expected. The fitting parameter $\alpha$ is between 8 - 10, i.e., a bit higher than the values for the phantom which are between 5 - 7. This difference may occur because in the in vivo experiment, other factors such as metabolism, blood flow, or breathing are included in the values obtained.

\subsubsection{Diversity of the Channel}

The diversity of the channel in transmission and reception is assessed throughout this section. For this purpose, the correlation coefficients for both experimental measurement setups are computed as Equation (4.2). Since the number of in-body locations on the in vivo experiment is very limited, only the correlation in reception could be evaluated in this case.

As receiving antenna, the slot antenna used in the previous sections is chosen, whereas the CPW miniaturized antenna acts as the transmitter for all the scenarios explained hereon.

\subsubsection{Correlation in Transmission}

\section{Phantom-based Scenario}

In order to evaluate the diversity of the channel in transmission, the correlation coefficients are computed from the values obtained in the phantom-based setup from 3.1 to $5.1 \mathrm{GHz}$ described in Section 4.3.1.1 particularized for the slot patch antenna. The VNA and the positioner configuration is detailed in Table 4.4.

The channel impulse response in a reference location of the in-body transmitter is correlated with those located in the same $\mathrm{Z}$ plane $(\mathrm{Z}=0)$ as well as at different heights $(Z=\Delta z, Z=2 \Delta z)$ to analyze the correlation in transmission. The location of the reference impulse response is set at the center of XY plane at $\mathrm{Z}=0$ (see Figure 4.13). 
From the correlation coefficients, the probability that these coefficients are equal to or greater than 0.8 is calculated. In Table 4.8 , the probability values for each subband and for the entire frequency range are shown. For each of these values, different areas are considered (see Figure 4.13). The first value takes into account the correlation coefficients from $\mathrm{X}=0$, i.e., the closest locations between antennas, to $\mathrm{X}=3$ ( $1^{\text {st }}$ half $)$, where the reference position is located, and all antenna locations in $\mathrm{Y}$ axis. The second one considers positions from $\mathrm{X}=3$ to $\mathrm{X}=6$ ( $2^{\text {nd }}$ half $)$ and all antenna locations in $\mathrm{Y}$ axis as well. Finally, the last value assumes all antenna locations in the same XY plane.

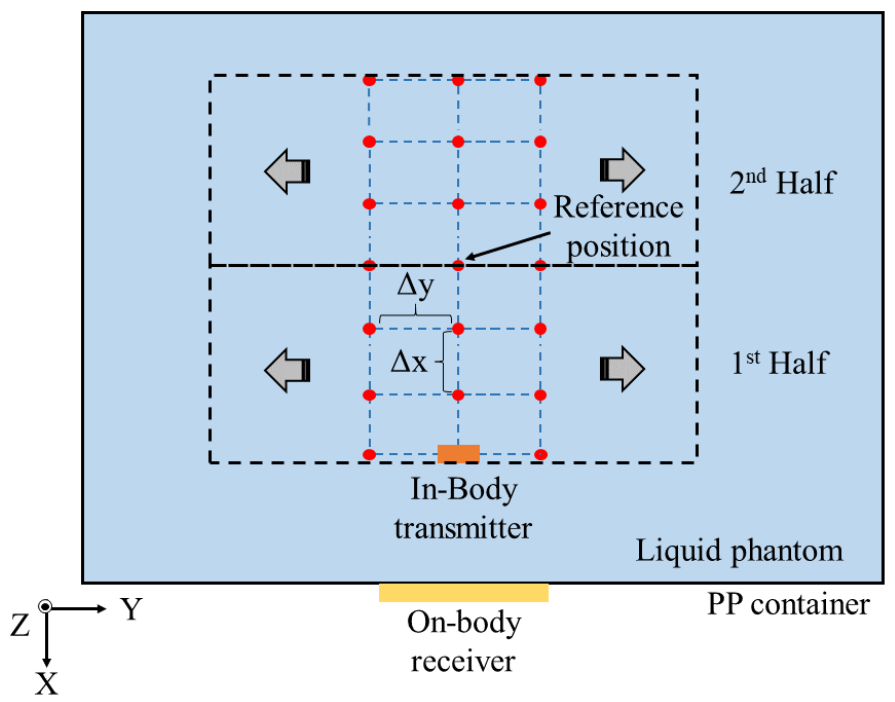

Figure 4.13: Measuring points considered in each half for the calculation of correlation coefficients in transmission for the phantom-based measurements

From Table 4.8, one can observe that the correlation is getting lower as the distance between in-body transmitters increases in $\mathrm{Z}$ axis for all the cases, as concluded in [41]. Besides, the correlation is lower for those locations farther in $\mathrm{X}$ axis. This decrement is influenced by multipath effect due to the multiple bounces on the walls of the box. In Figure 4.14, it can be observed that the main contribution begins to be comparable to others. The variations in $\mathrm{Z}=0$ can be caused by the unpredictable multipath effect. Moreover, the correlation per subband is higher when the subband is located at upper frequencies whatever the distance between transmitters in $\mathrm{Z}$ axis is. Regarding the correlation 
Table 4.8: Probability for correlation coefficients $\geq 0.8$

\begin{tabular}{|c|c|c|c|}
\hline $\begin{array}{c}\text { Frequency range } \\
(\mathrm{GHz})\end{array}$ & $\begin{array}{c}\mathrm{Z}=0 \\
1^{\text {st }} \text { Half } / 2^{\text {nd }} \text { Half/ } / \\
\text { Whole }\end{array}$ & $\begin{array}{c}\mathrm{Z}=\Delta \mathrm{z} \\
1^{\text {st }} \text { Half } / 2^{\text {nd }} \text { Half/ } \\
\text { Whole }\end{array}$ & $\begin{array}{c}\mathrm{Z}=2 \Delta \mathrm{z} \\
1^{\text {st }} \text { Half } / 2^{\text {nd }} \text { Half } / \\
\text { Whole }\end{array}$ \\
\hline $3.1-3.6$ & $0.667 / 0.286 / 0.531$ & $0.524 / 0.191 / 0.469$ & $0.381 / 0.143 / 0.347$ \\
\hline $3.6-4.1$ & $0.714 / 0.191 / 0.551$ & $0.714 / 0.333 / 0.510$ & $0.571 / 0.191 / 0.429$ \\
\hline $4.1-4.6$ & $0.809 / 0.238 / 0.592$ & $0.714 / 0.333 / 0.571$ & $0.619 / 0.333 / 0.531$ \\
\hline $4.6-5.1$ & $0.857 / 0.667 / 0.796$ & $0.857 / 0.619 / 0.755$ & $0.762 / 0.524 / 0.694$ \\
\hline $3.1-5.1$ & $0.667 / 0.238 / 0.512$ & $0.667 / 0.191 / 0.490$ & $0.619 / 0.095 / 0.429$ \\
\hline
\end{tabular}

considering the whole frequency band, it decreases as the distance between antennas increases in height as well. Besides, the correlation values considering the entire bandwidth are close to those obtained at the lower subbands for $\mathrm{Z}$ $\leq \Delta \mathrm{z}$. However, the correlation in the lowest subband is quite lower than the rest of cases at $\mathrm{Z}=2 \Delta \mathrm{z}$.

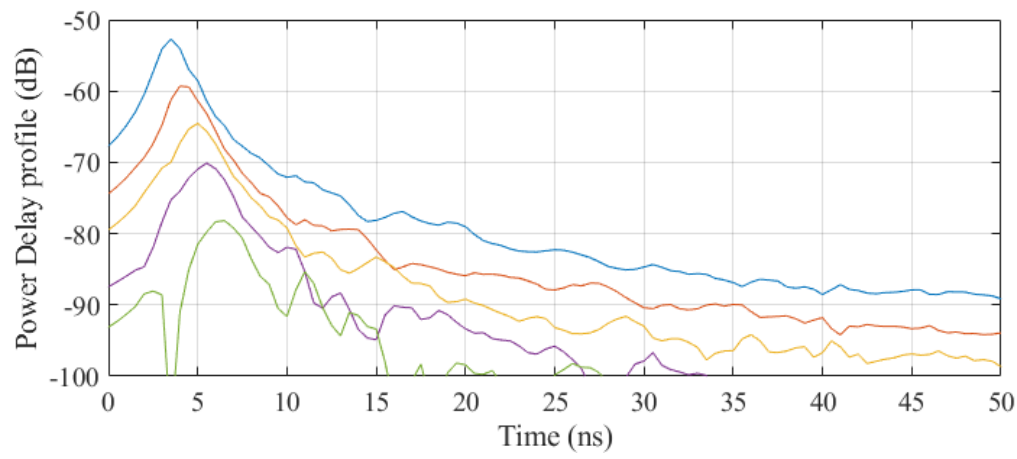

Figure 4.14: Power delay profile for 5 different depths in the $\mathrm{X}$ axis considering RX1.

\subsubsection{Correlation in Reception}

The correlation in reception is evaluated by the correlation coefficients as well. Here, the diversity of the channel could be assessed for both experimental scenarios, i.e., phantom and in vivo. 


\section{Phantom-based Scenario}

For the evaluation of the correlation in reception for the phantom-based measurement setup, the correlation coefficients are computed from the values obtained in the phantom-based setup from 3.1 to $5.1 \mathrm{GHz}$ described in Section 4.3.1.1 particularized for the slot patch antenna. In this case, the slot antenna is located over the external's container wall at different locations (see Figure 2.14). The on-body antenna is located in an initial position (in orange color in Figure 4.15) and moved away in steps of the wavelength $(2 \lambda$ and $4 \lambda)$. In every location of the receiving antennas, the submerged transmitter into the liquid phantom is moved into a XYZ mesh of $7 \times 7 \times 3$ as Section 4.3.1.1.

It is worth mentioning that the locations of the receiving antennas are selected according to the location of the human and pig small bowel (see Figure 2.14). As can be observed in Figure 2.14, the small intestine is located at the bottom left side of the belly. In this experiment, the on-body antenna is accordingly placed in order to try to be close to this area.

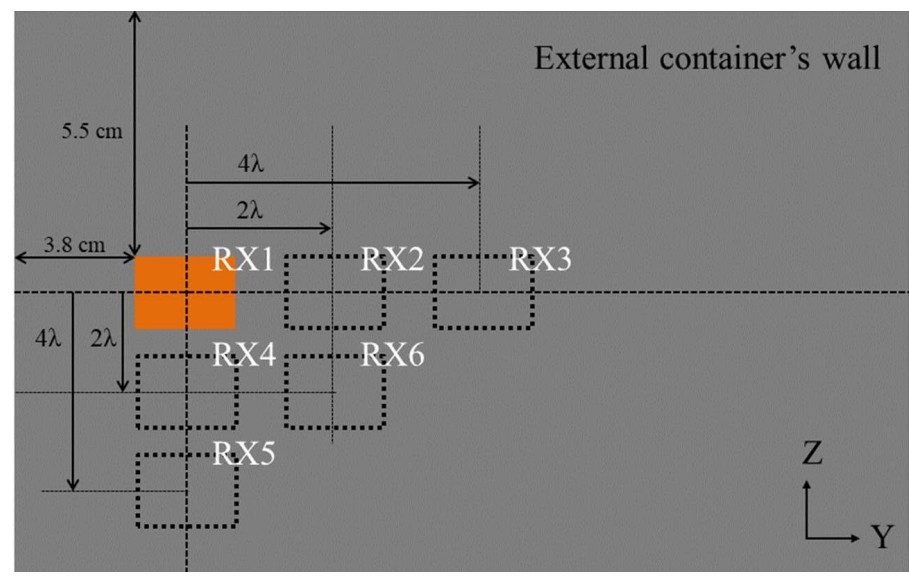

Figure 4.15: Locations of on-body antenna to study the correlation in reception in the IB2OB phantom-based scenario

In accordance with Equation (4.2), $h_{d_{1}}(\tau)$ is the channel impulse response obtained from RX1 (see Figure 4.15) in a transmitting antenna location, whereas $h_{d_{2}}(\tau)$ is the channel impulse response in the same in-body antenna location obtained from other receivers. RX2 and RX4 are $2 \lambda$ away from RX1 in the $\mathrm{Y}$ and $\mathrm{Z}$ axes, respectively, whereas RX3 and RX5 are at $5 \lambda$. On the other hand, $\mathrm{RX} 6$ is $2 \lambda$ away in $\mathrm{Z}$ and $\mathrm{Y}$ axes. 


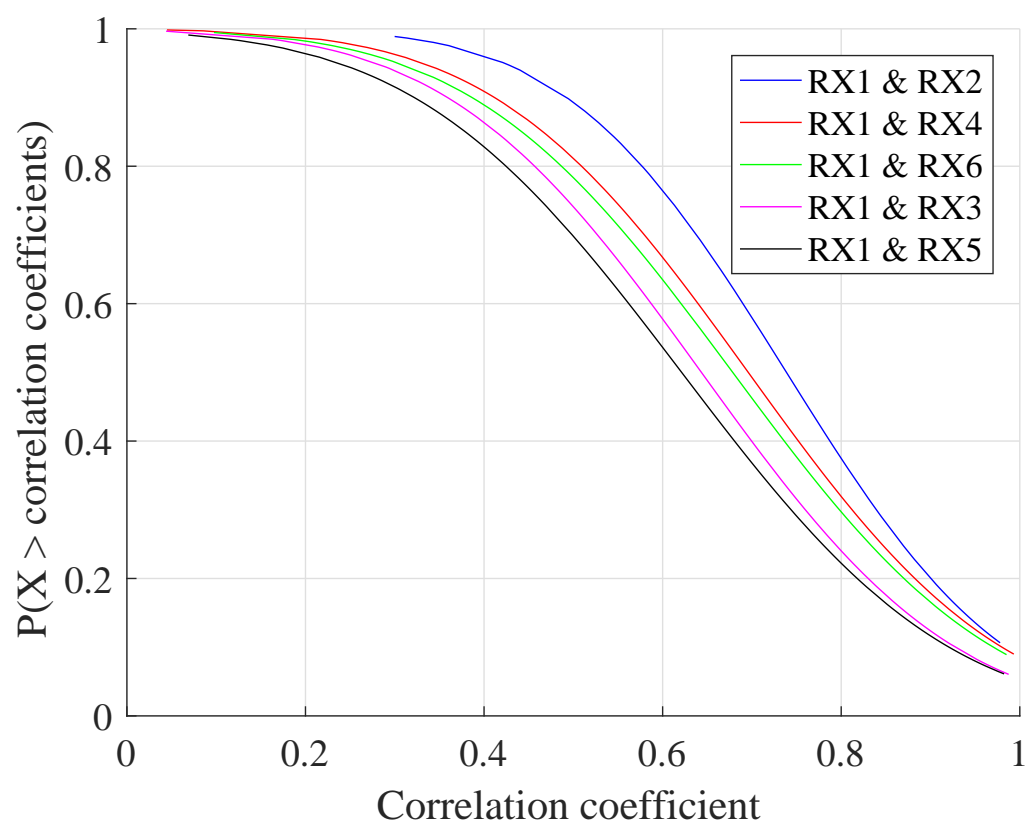

Figure 4.16: Complementary cumulative distribution function (CCDF) for correlation coefficients in reception.

Figure 4.16 shows the CCDF for the correlation in reception considering the whole available bandwidth $(3.1-5.1 \mathrm{GHz})$. The CCDF is computed from all the XYZ measurement points. As can be seen, the correlation is quite high when the receiving antennas are closer in the $\mathrm{Y}$ and $\mathrm{Z}$ axes. A slight decrease can be seen when the receiving antennas are separated $2 \lambda$ in the $\mathrm{Z}$ axis. The correlation between RX1 and RX6 is lower than those values for RX2 and RX4. On the other hand, the correlation considerably decreases as the distance between receivers is long, i.e., more than $2 \lambda$. As can be observed, the correlation is lower when the receivers are located at different $\mathrm{Z}$ planes. 


\section{In vivo Scenario}

For the in vivo experiment, the correlation coefficients are calculated as in the previous section. According to Figure 2.17 and Equation (4.2), two tables of values are obtained. For the values shown in Table 4.9 and Table 4.10, the reference channel impulse response is obtained from the receiving antenna location number 7 (see Figure 2.16). For both tables, the reference channel impulse is correlated with the channel impulse responses obtained by the other receiving antenna locations. In Table 4.9, the channel impulse responses are obtained from the same in-body antenna location. On the other hand, in Table 4.10, the channel impulse response obtained from the receiving antenna location number 7 at the position of the implanted antenna shown in Figure 4.11(a) is correlated with those obtained by all the receivers at the position of the implanted antenna shown in Figure 4.11(b). The configuration of the involved pieces of equipment are detailed in Section 2.3.2.1.

Table 4.9: Correlation coefficients when the in-body antennas are located in the same location

\begin{tabular}{|c|c|c|c|c|c|}
\cline { 3 - 5 } \multicolumn{1}{c|}{} & $\mathrm{Y}=-5 \lambda$ & $\mathrm{Y}=-2 \lambda$ & $\mathrm{Y}=0$ & $\mathrm{Y}=2 \lambda$ & $\mathrm{Y}=5 \lambda$ \\
\hline $\mathrm{X}=-5 \lambda$ & & & 0.3573 & & \multicolumn{1}{c|}{} \\
\hline $\mathrm{X}=-2 \lambda$ & & 0.803 & 0.951 & 0.851 & \\
\hline $\mathrm{X}=0$ & 0.9042 & 0.976 & 0.976 & 0.885 & 0.8823 \\
\hline $\mathrm{X}=2 \lambda$ & & 1 & 0.917 & 0.857 & \\
\hline $\mathrm{X}=5 \lambda$ & & & 0.890 & & \\
\hline
\end{tabular}

Table 4.10: Correlation coefficients when the in-body antennas are located in different location

\begin{tabular}{|c|c|c|c|c|c|}
\cline { 3 - 6 } \multicolumn{1}{c|}{} & $\mathrm{Y}=-5 \lambda$ & $\mathrm{Y}=-2 \lambda$ & $\mathrm{Y}=0$ & $\mathrm{Y}=2 \lambda$ & $\mathrm{Y}=5 \lambda$ \\
\hline $\mathrm{X}=-5 \lambda$ & & & 0.3387 & & \multicolumn{1}{c|}{} \\
\hline $\mathrm{X}=-2 \lambda$ & & 0.5893 & 0.3485 & 0.5571 & \\
\hline $\mathrm{X}=0$ & 0.6568 & 0.6276 & 0.5004 & 0.4049 & 0.3823 \\
\hline $\mathrm{X}=2 \lambda$ & & 0.7924 & 0.6358 & 0.6061 & \\
\hline $\mathrm{X}=5 \lambda$ & & & 0.8369 & & \\
\hline
\end{tabular}

From the results shown in Table 4.9 and Table 4.10 , it can be noted that the correlation in reception decreases as the distance between receivers is higher. However, the correlation is quite high when the transmitting antenna is placed 
in the same location. When the transmitting antenna location is varied, the correlation is significantly reduced. 


\section{Chapter 5}

\section{Diversity Techniques}

The main drawback of the radio wave transmission through the human body is the high attenuation introduced by the living body tissues. Besides, this attenuation dramatically increases with the increment in frequency. As mentioned in previous chapters, these losses constrain both the distance between transmitter and receiver as well as the available bandwidth to transmit efficiently at UWB frequencies. Therefore, novel healthcare applications at UWB frequency band would depend on the performance of the radio channel.

In this chapter, methods based on channel diversity are applied in this complex environment to achieve a better channel performance. 


\subsection{Introduction}

\subsubsection{Time Reversal Technique}

\subsubsection{Principle}

Although Time Reversal (TR) signal processing was intended for acoustics [89], it was extended to electromagnetic waves [90] in 2004. In the research field of the wireless radio propagation, this technique was taken to focus the transmitted energy on the intended receiver, thus reducing the interference from other field resources. This technique might be very useful for multireceiver communication systems with a large number of involved nodes [91].

Basically, TR achieves a space-time compression [92]. The main idea is to precode the transmitted UWB pulse to obtain a new channel impulse response. Firstly, the receiver sends pulse to the transmitter which is known by the transmitter and receiver. Then, from such received pulse, the transmitter can estimate the channel impulse response, $h(\tau)$, between the transmitter and receiver. Thus, the transmitter sends a precoded signal to the receiver, which consists of the signal to be transmitted, $x(\tau)$, convolved with the inverse and conjugated channel impulse response previously estimated, $h^{*}(-\tau)$. Accordingly, it should be remarked that for applying this technique the channel should be reciprocal so that the channel impulse response can be estimated on the transmitter side [90].

Mathematically, the received signal, $y(\tau)$, can be expressed as:

$$
y(\tau)=\left(x(\tau) \otimes h^{*}(-\tau)\right) \otimes h(\tau)=x(\tau) \otimes\left(h^{*}(-\tau) \otimes h(\tau)\right)=x(\tau) \otimes h_{e q}(\tau)
$$

where $\otimes$ is the convolution operator and $h_{e q}(\tau)$ is the equivalent channel impulse response obtained from:

$$
h_{e q}(\tau)=h^{*}(-\tau) \otimes h(\tau)
$$

This is the autocorrelation of the channel impulse response. It should be also considered to normalize $h_{e q}(\tau)$ in order to compare this technique with other schemes [92],

$$
h_{e q}(\tau)=\frac{h^{*}(-\tau)}{\sqrt{\int\left|h^{*}(-\tau)\right|^{2}}} \otimes h(\tau) .
$$


In Figure 5.1, the scheme of TR technique applied to a multi-receiver system is shown. In this case, the equivalent impulse response is only optimal for TX$\mathrm{RX}_{1}$ due to the use of the estimated inverse and conjugated channel impulse response of $h_{1}^{*}(\tau)$ as precoding signal.

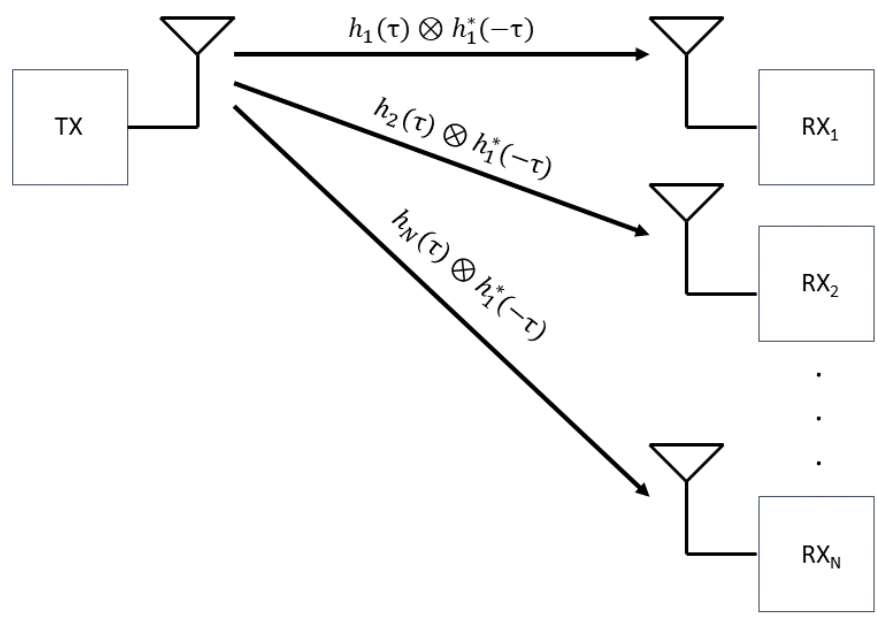

Figure 5.1: Scheme of TR technique for a multi-receiver system

To evaluate the characteristics and performance of the TR technique in a specific application, some figures of merit can be found in the literature. In [92], authors proposed a peak to peak gain $\left(G_{p 2 p}\right)$ which shows the ratio between the strongest path using $\operatorname{TR}\left(h_{e q}\right)$ and the conventional form $(h)$. So, this is worth mentioning that TR could achieve a gain on the receiver side precoding the transmitted signal of the intended user. It can be measured as follows:

$$
G_{p 2 p}=10 \log _{10}\left(\frac{\max \left(\left|h_{e q}(\tau)\right|^{2}\right)}{\max \left(|h(\tau)|^{2}\right)}\right)
$$

In the case of the receiver captures all the energy from the channel response, then the gain is defined as:

$$
G_{p 2 p}=10 \log _{10}\left(\frac{\int\left|h_{e q}(\tau)\right|^{2}}{\int|h(\tau)|^{2}}\right)
$$




\subsubsection{Maximal-Ratio Combining}

\subsubsection{Principle}

Methods based on diversity combining can cope with the high attenuation that living tissues have at high frequencies. In an IB2OB scenario where the information among sensors is wirelessly sent, there are usually involved one implanted transmitter such as the capsule endoscope or other bioimplant, and an array of multiple sensors over the human torso. Taking into account this typical scenario, Maximal-Ratio Combining (MRC) can profit from the receiver diversity in order to enhance the channel performance. This technique tries to combat the multiple fading by combining the SNR of each received branch [93]. Here, branch refers to the channel impulse response between the transmitter and each of the on-body receivers. On the receiver side, the received signals for each receiver antenna are combined to increase the SNR of the whole system and thus reducing the Bit Error Rate (BER). Before combining, the received signals are multiplied by a weightening factor in every branch. The values of the weights are such that they maximize the output SNR. The weights are the complex conjugate of the channel impulse response obtained in each receiver, i.e., $w_{i}=h_{i}^{*}(\tau)$ being $w_{i}$ the weight of the receiver $i$. The architecture of the MRC technique is depicted in Figure 5.2.

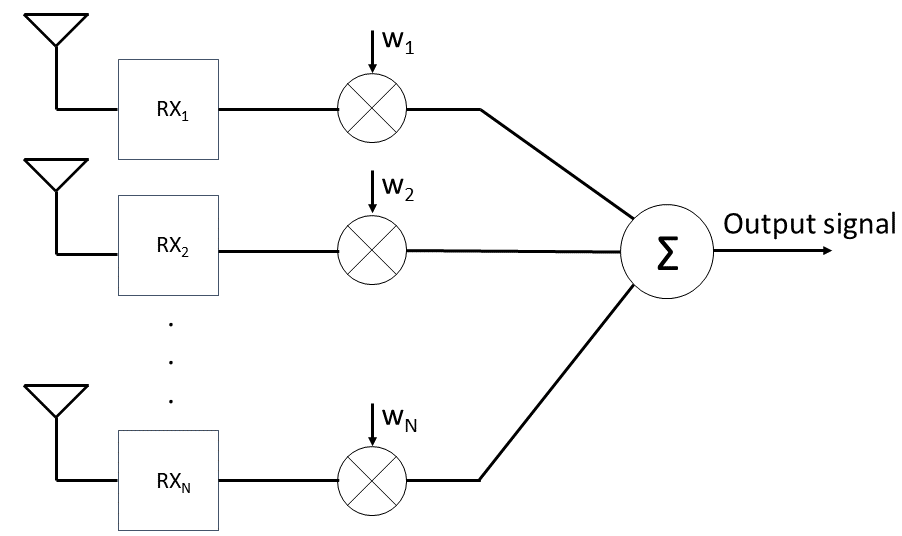

Figure 5.2: Architecture of a receiver using MRC technique 
According to the scheme of MRC depicted in Figure 5.2, the combined output signal, $r(\tau)$, is given by [94]:

$$
\begin{aligned}
\mathbf{y}(\tau) & =\mathbf{h}(\tau) x(\tau)+\mathbf{n}(t), \\
\mathbf{h} & =\left[h_{1}, h_{2}, \ldots, h_{N}\right]^{T}, \\
\mathbf{n} & =\left[n_{1}, n_{2}, \ldots, n_{N}\right]^{T}, \\
r(\tau) & =\mathbf{w}^{H} \mathbf{y}=\mathbf{w}^{H} \mathbf{h} x(\tau)+\mathbf{w}^{H} \mathbf{n},
\end{aligned}
$$

where $\mathbf{y}(\tau)$ is the received signal at the array of $N$ elements, $\mathbf{h}(\tau)$ is the channel impulse response for each branch, $x(\tau)$ is the transmitted signal and $\mathbf{n}$ is the noise. Since the transmitted signal $x(\tau)$ has unit average power, the instantaneous output SNR will be:

$$
S N R=\frac{\left|\mathbf{w}^{H} \mathbf{h}\right|^{2}}{E\left[\left|\mathbf{w}^{H} \mathbf{n}\right|^{2}\right]}
$$

The term of the denominator is the noise power given by:

$$
\begin{aligned}
E\left[\left|\mathbf{w}^{H} \mathbf{n}\right|^{2}\right] & =E\left[\left|\mathbf{w}^{H} \mathbf{n} \mathbf{n}^{H} \mathbf{w}\right|^{2}\right]=\sigma^{2} \mathbf{w}^{H} I_{N} \mathbf{w} \\
& =\sigma^{2} \mathbf{w}^{H} \mathbf{w}=\sigma^{2}\|\mathbf{w}\|^{2}
\end{aligned}
$$

being $I_{N}$ the identity matrix $\mathrm{N} \times \mathrm{N}$. Considering that the weight which maximizes the output SNR is the complex conjugate values obtained in each receiver, $h_{i}^{*}(\tau)$, the ouput SNR is therefore given by:

$$
S N R=\frac{\left|\mathbf{h}^{H} \mathbf{h}\right|^{2}}{\sigma^{2} \mathbf{h}^{H} \mathbf{h}}=\frac{\mathbf{h}^{H} \mathbf{h}}{\sigma^{2}}=\sum_{i=1}^{N} \frac{\left|h_{n}\right|^{2}}{\sigma^{2}}
$$

\subsubsection{Reconstruction of the Channel Impulse Response. CLEAN algorithm}

Considering the weight values on the receiver side described in Section 5.1.2.1, the channel impulse response of each receiver should be known. A UWB received signal consists of a transmitted UWB pulse, the channel impulse response and the noise. In the literature, there are available algorithms to deduce the Multipath Components (MPCs) in the context of UWB systems [19]. For example, the SAGE algorithm performs a iterative determination of the maximum-likelihood of the parameters of the MPCs [95]. 
One of the most extended high-resolution algorithm to extract the MPCs with a certain accuracy is the CLEAN algorithm [96]. The premise of this algorithm is that the received signal is a sum of scaled and delayed replicas of the known transmitted pulse. This algorithm has been used in works by using computer anatomical human body models [97]. The channel impulse response is given by:

$$
h(\tau)=\sum_{i=1}^{N} A_{k} \delta\left(\tau-\tau_{i}\right),
$$

where $A_{k}$ and $\tau_{i}$ are the amplitude and time delay of the $i$ th MPCs, respectively, and $N$ is the total number of considered MPCs. The CLEAN algorithm first finds the largest pulse correlating the received signal with the transmitted pulse, thus identifying the highest peak. The amplitude, $\mathrm{A}_{T_{n}}$, and time delay, $\tau_{n}$, of this peak is recorded and then its power contribution is subtracted from the received signal at its corresponding time delay. This process is iteratively repeated until the difference between the amplitude of the highest subtracted peak and the current extracted contribution are below certain threshold $\gamma$. In Figure 5.3, the scheme of CLEAN algorithm is depicted.

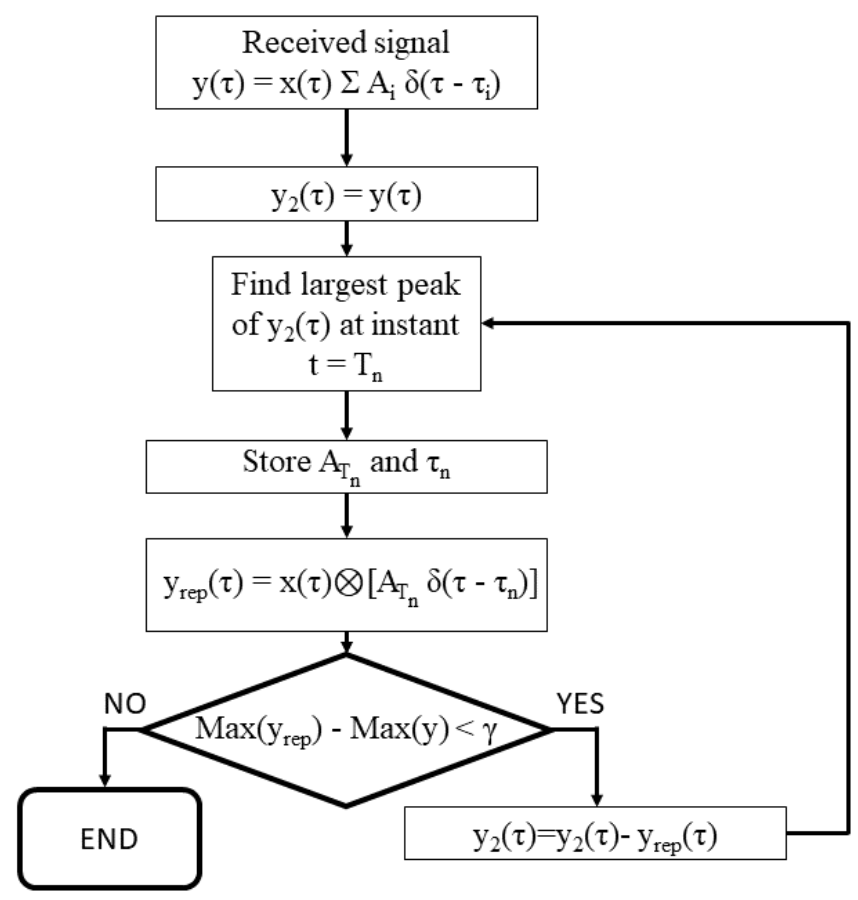

Figure 5.3: CLEAN algorithm 


\subsection{Time Reversal for In-Body to In-body Communications}

As mentioned, the antennas involved in an IB2IB scenario are all implanted inside the body. Since the losses in the propagation medium can dramatically increase at high frequencies, this scenario can be the the intermediate step in the connection between a deeply implanted node and an external one. This intermediate propagation scenario could arise between the deeply implanted node and a subcutaneous one.

Throughout this section, the TR technique is tested to be used in medical applications. Specifically, this technique is applied to improve the received power in an IB2IB scenario. This fact can improve the radio link established among implanted sensors, compensating the high losses of the propagation medium at gigahertz frequencies.

\subsubsection{Configuration $\mathcal{G}$ Methodology}

As a first analysis of TR signal processing for in-body communications, this technique is tested in an IB2IB scenario. Here, the transmitted signal by the in-body transmitter is precoded and the gain on the receiver side is quantified. Specifically, TR technique is applied in the channel measurement campaign described in Section 4.2.2 considering the receiver RX1 (see Figure 4.4). The configuration of the VNA and the positioner is shown in Table 4.2. In this measurement campaign, two identical UWB monopole antennas were chosen. These are two miniaturized in-body antennas described in Section 3.2.2. Both antennas are depicted in Figure 4.1.

Regarding the data processing, the performance of TR technique is assessed by means of the $G_{p 2 p}$ described in Equation (5.5). As mentioned in Section 5.1.1.1, the reciprocity of the channel must be ensured in order to apply this technique. Therefore, the reciprocity of the channel is previously evaluated in the following section. 


\subsubsection{Reciprocity of the Propagation Channel}

A reciprocal channel means that the forward transmission coefficients $\left(\mathrm{S}_{21}\right.$, $\mathrm{S}_{12}$ ) have similar features in both communication directions. When both antennas are located in free space with Line-Of-Sight (LOS) conditions, it is relatively obvious to think that the channel is reciprocal. In in-body communications, the antennas are located into the body, which produces a high variation in their characteristics. The complex permittivity of body tissues modifies the propagation speed and therefore the wavelength compared to this one in free space. Thus, the field regions might change their radiation areas [60]. So, the antennas could be in near field regions where the radiation parameters could fluctuate considerably. On the other hand, the propagation medium is not the free space and the conditions, where the transmitter and the receiver have in their locations, are not the same.

Figure 5.4 depicts the $\mathrm{S}_{21}$ and $\mathrm{S}_{12}$ in three different measurement points at different heights. It can be noted that the frequency responses are practically identical to each other. Besides, Table 5.2 shows the probability values that the correlation coefficients between $\mathrm{S}_{21}$ and $\mathrm{S}_{12}$ in the same measurement point at different $\mathrm{Z}$ planes are above 0.8 . One can observe the high similarity regardless the location of the transmitting antenna. Hence, it can be concluded that the propagation channel can be practically considered reciprocal when both antennas are submerged into the liquid phantom.

Table 5.1: Probability that correlation between $S_{21}$ and $S_{12}$ is greater than 0.8 .

\begin{tabular}{cc}
\hline \hline Z plane & Probability $>\mathbf{0 . 8}$ \\
\hline$-2 \Delta z$ & 0.8571 \\
\hline$-\Delta z$ & 0.9310 \\
\hline 0 & 0.9355 \\
\hline$\Delta z$ & 0.9032 \\
\hline $2 \Delta z$ & 0.800 \\
\hline \hline
\end{tabular}




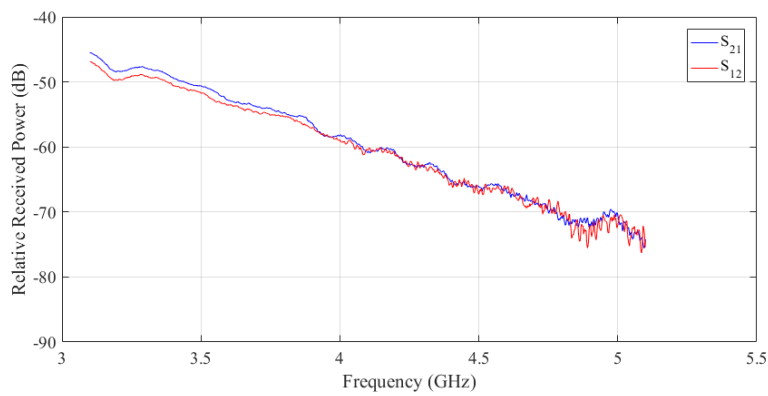

(a)

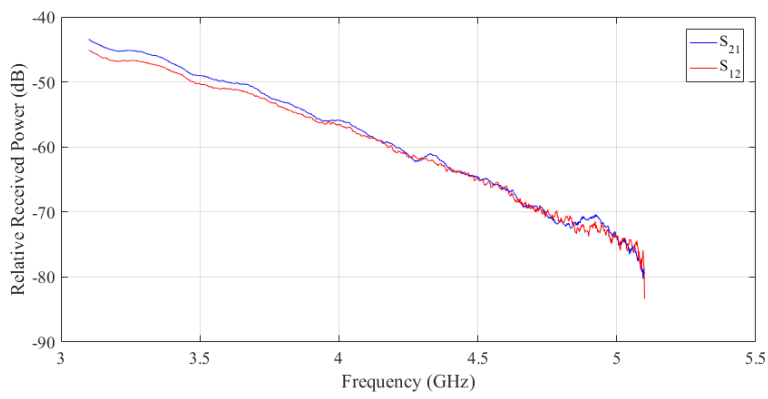

(b)

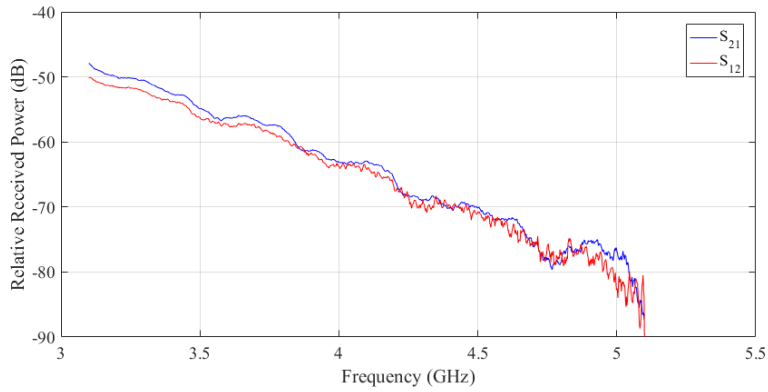

(c)

Figure 5.4: Forward transmission coefficient in both directions (from port 1 to port 2 and viceversa) in three transmitting antenna locations at different $\mathrm{Z}$ planes. $\mathrm{Z}=-\Delta \mathrm{z}$ (a), $\mathrm{Z}=0$ (b) and $\mathrm{Z}=\Delta \mathrm{z}(\mathrm{c})$. 


\subsubsection{Results}

In this section, the $G_{p 2 p}$ is calculated for each $\mathrm{Z}$ plane to evaluate the performance of $\mathrm{TR}$ in this specific application. In each measurement point, the transmitted signal is precoded by the channel impulse response obtained on the transmitter side in this point. As in Section 4.2.2, the plane $\mathrm{Z}=0$ is the center plane, whereas the other planes were located above $(-\Delta z,-2 \Delta z)$ and below $(\Delta \mathrm{z}, 2 \Delta \mathrm{z})$ from $\mathrm{Z}=0$.

As evidenced in Section 5.2.2, a reciprocal channel can be assumed. Hence, according to Equation (5.5), the estimated channel impulse response $h^{*}(-\tau)$ is calculated from the $S_{12}$ samples, whereas $h(\tau)$ is the channel impulse response obtained from $S_{21}$ samples.

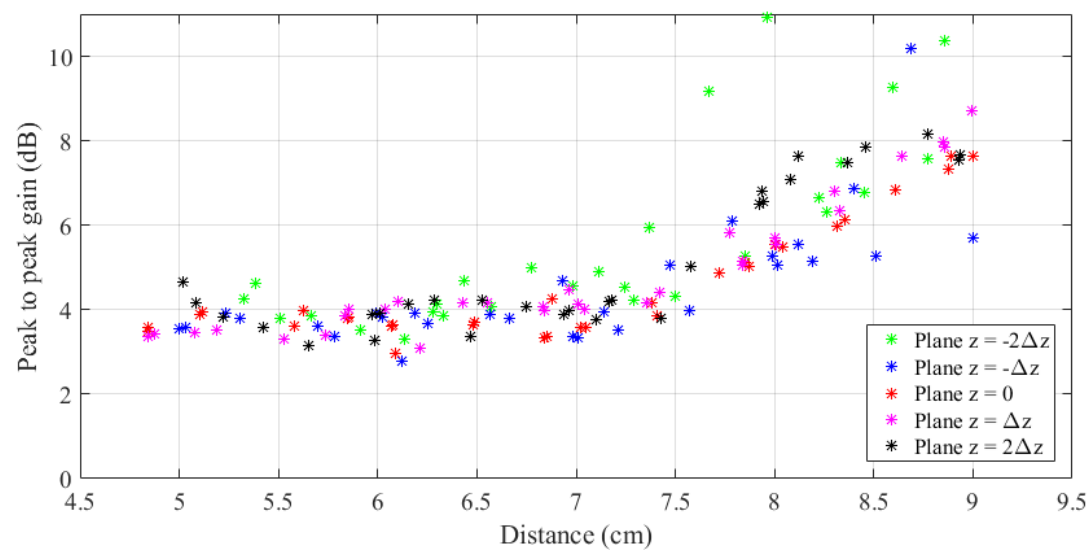

Figure 5.5: Peak to peak gain at different $Z$ planes

Figure 5.5 shows the values of $G_{p 2 p}$ for each Z plane. As can be observed, the gain approximately varies from 4 to $11 \mathrm{~dB}$, being higher when the implanted transmitter and receiver are further away from each other. In addition, the gain has higher values when the transmitter is located at the upper and lower planes, i.e., $(-2 \Delta z, 2 \Delta z)$ where the received power is lower. Section 4.2.2 shows the losses in the liquid phantom within a distance between antennas from 4.8 to $9 \mathrm{~cm}$. As can be observed in Figure 4.5 of Section 4.2.2, the losses are $5 \mathrm{~dB} / \mathrm{cm}$, approximately. From the results obtained applying the TR technique shown in Figure 5.5, the distance between transmitters could be roughly increased from 1 to $2 \mathrm{~cm}$. As mentioned, this increase is greater when the antennas are farther apart. It implies $11-22 \%$ of that without using TR signal processing. On the 
other hand, it is worth mentioning the impact of the diversity of the channel in the performance of TR. Observing the diversity in transmission depicted in Figure 4.7 of Section 4.2 .2 , it can be noted that there is a relation between these values and the $G_{p 2 p}$. From this figure, it can be concluded that $G_{p 2 p}$ increases when the correlation decreases at the furthest distances.

\subsection{MRC Technique for In-Body to On-body Communications}

Throughout this section, the MRC technique is tested to be used in IB2OB applications where a multi-receiver system is usually used. This technique is applied to improve the channel performance in this particular scenario.

\subsubsection{Measurement setup 69 Methodology}

As an initial approach, the MRC technique is tested in an IB2OB scenario. Accordingly, MRC is applied in the phantom-based measurement campaign explained in Section 4.3.1.1 particularized for the on-body receiving slot patch antenna described in Section 3.3.3. In that measurement campaign, the miniaturized CPW-fed UWB monopole antenna outlined in Section 3.2.2 acted as in-body transmitter. The VNA and positioner configuration is summarized on Table 4.4. In Section 4.3.1.1, the in-body transmitter is placed at $3 \mathrm{Z}$ planes separated steps of $\Delta z$ to each other. The plane $\mathrm{Z}=0$ is the center plane, where the transmitter and the receiver are aligned in height, whereas the other planes are located above $(\Delta z, 2 \Delta z)$ from this plane.

In order to make a more realistic study, the channel impulse response and its conjugate are not obtained in the same way. On the receiver side, the received signal is obtained and the channel impulse response is reconstructed from the transmitted and received pulses. The conjugate for the weight values is deduced from the channel estimation with the CLEAN algorithm explained below, whereas the channel impulse response is measured by the VNA. 


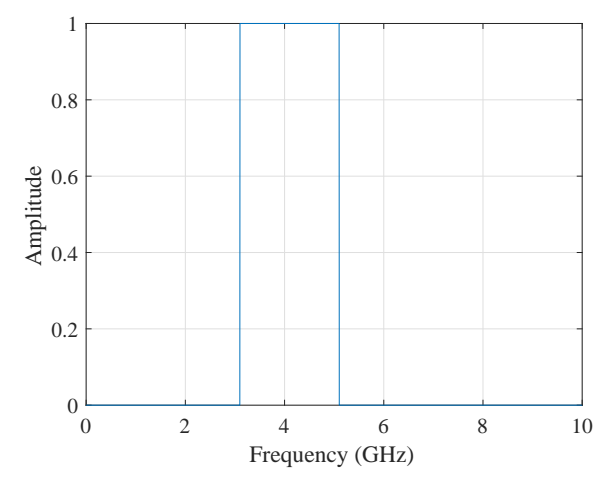

(a)

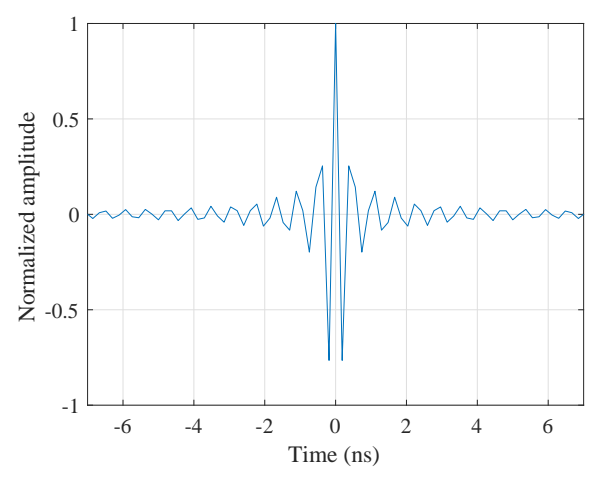

(b)

Figure 5.6: Transmitted signal in frequency (a) and time (b) domain

\subsubsection{Threshold Selection for CLEAN Algorithm}

As mentioned in Section 5.1.3, CLEAN algorithm stops the iterative process when a certain threshold, $\gamma$, is reached. In this section, the optimal threshold is sought to reconstruct the channel impulse response efficiently.

This algorithm extracts the tap delay profile by knowing the transmitted pulse. As a first approach, here a rectangular waveform which covers the whole available bandwidth (3.1 - 5.1) is chosen. In Figure 5.6, the transmitted pulse in the frequency and time domains are depicted.

In order to get the optimal threshold for the CLEAN algorithm, the channel impulse responses obtained from the measurement campaign described in Section 4.3.1.1 are used. Specifically, the channel impulse response is obtained when the in-body transmitter is located at $\mathrm{X}=4, \mathrm{Y}=0, \mathrm{Z}=1$, i.e., at the center point of the XYZ mesh, and the on-body received is placed in the middle of the external container wall $(\mathrm{Y}=0, \mathrm{Z}=0)$. In order to measure the accuracy of the algorithm, the correlation coefficients between the reconstructed and the original received signal are obtained for different threshold values. In Table 5.2, the correlation coefficient for 5 different thresholds are shown. As can be noted, the correlation increases as the threshold grows up to $10 \mathrm{~dB}$. From this value, the correlation decreases because the algorithm becomes less efficient. This is due to the influence of the bandwidth of the pulse on the effectiveness of the CLEAN algorithm. In Figure 5.7, one can observe the loss of efficiency of the CLEAN algorithm above $10 \mathrm{~dB}$. 
Table 5.2: Correlation values for different thresholds

\begin{tabular}{cc}
\hline \hline Threshold $(\gamma)$ & Correlation coefficient \\
\hline $3 \mathrm{~dB}$ & 0.665 \\
\hline $5 \mathrm{~dB}$ & 0.815 \\
\hline $10 \mathrm{~dB}$ & 0.966 \\
\hline $15 \mathrm{~dB}$ & 0.574 \\
\hline $20 \mathrm{~dB}$ & 0.447 \\
\hline \hline
\end{tabular}

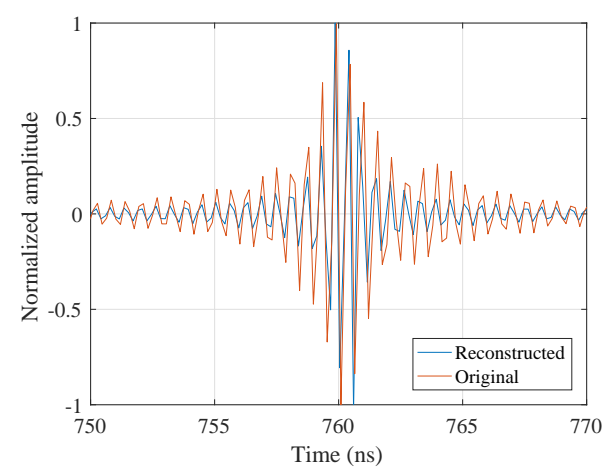

(a)

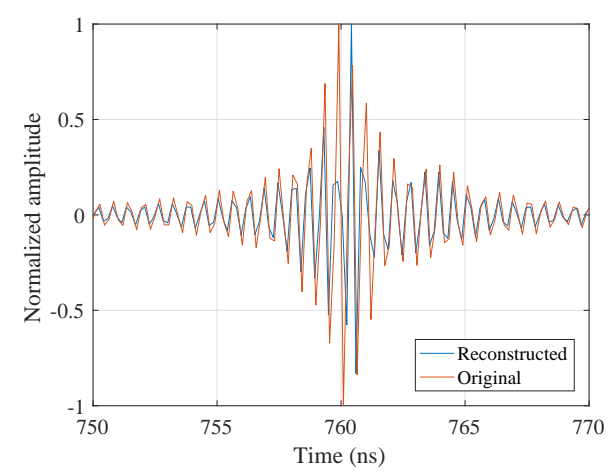

(b)

Figure 5.7: Original and reconstructed received signal using the CLEAN algorithm with a threshold of (a) $10 \mathrm{~dB}$ and (b) $20 \mathrm{~dB}$

\subsubsection{MRC Applied at In-body to On-body Communications}

As first analysis of MRC for IB2OB communications, this technique is applied in the IB2OB scenario explained in Section 4.3.1.1 particularized for the slot patch antenna. In addition to the central receiver, two other receivers are considered. The three receivers are placed at the middle of the container's wall $(\mathrm{Y}=0, \mathrm{Z}=0)$, at $-2 \lambda$ away in the $\mathrm{Y}$ axis $(\mathrm{Y}=-2 \lambda, \mathrm{Z}=0)$ and at $2 \lambda$ away in this axis as well $(\mathrm{Y}=2 \lambda, \mathrm{Z}=0)$, being $\lambda$ the effective wavelength at the central frequency of the bandwidth under analysis $(4.1 \mathrm{GHz})$ considering the propagation speed inside the phantom. 
According to the results of Section 5.3.2, the optimal value for the CLEAN algorithm in this particular case is $10 \mathrm{~dB}$. Therefore, this value of the threshold is chosen as stop criteria of the CLEAN algorithm.

In Figure 5.8, the gain in SNR in $\mathrm{dB}$ applying MRC with the three aforementioned receivers is shown. As can be observed, the gain is higher as the distance between antennas is higher. Besides, this increment is greater when the transmitter and receiver are located at different heights. The gain in SNR varies from 4 to $12 \mathrm{~dB}$, approximately. Considering the gain in SNR, it implies an increment on the distance between transmitter and receiver from 1 to $2 \mathrm{~cm}$. As mentioned, this increase is greater when the antennas are farther apart as well as at different heights. The distance between antennas could be enhanced from 11 to $22 \%$ by using MRC technique.

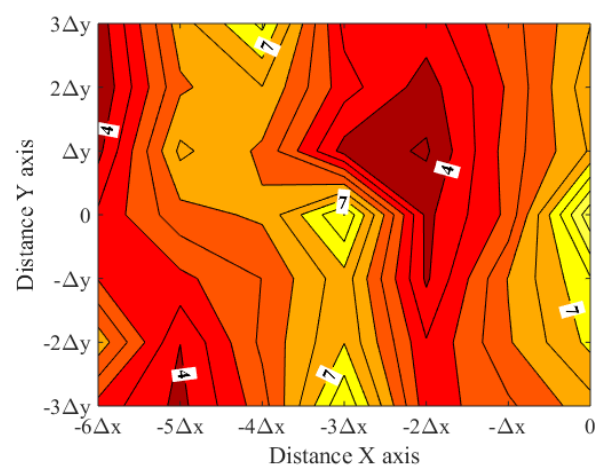

(a)

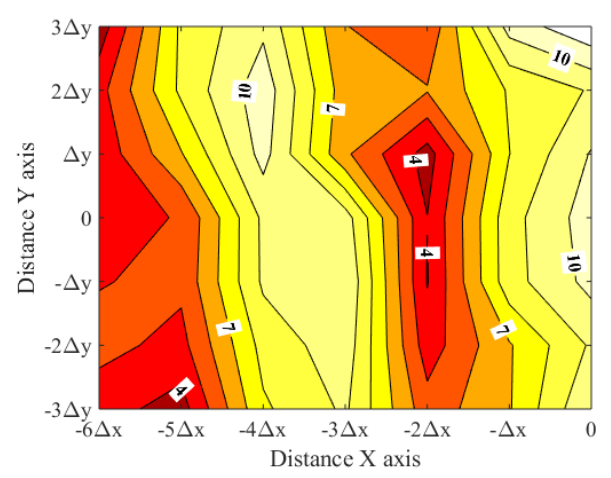

(b) 


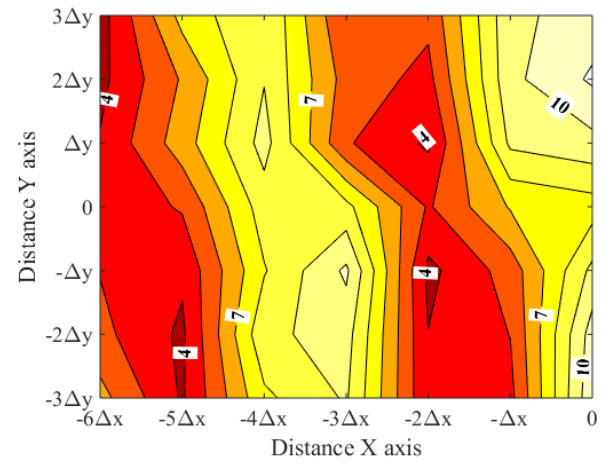

(c)

Figure 5.8: Gain in SNR applying MRC at different heights. (a) $\mathrm{Z}=0$, (b) $\mathrm{Z}=\Delta z$, (b) $\mathrm{Z}=2 \Delta z$ 



\section{Chapter 6}

\section{Conclusions and Perspective}

WBANs have a promising future. Many researchers are involved in the work to enhance the current in-body networks. They know that there is a gap to be filled.

The technology of wireless medical devices are far from the current advances in wireless communications. The current standard for in-body communications, IEEE 802.15.6, only allows the use of narrow band systems. Even though current wireless medical devices have a relative good penetration through human tissues and certain robustness, high data rate wireless connections are not possible given the limited available bandwidth. To solve this issue, UWB technology has emerged as a potential candidate for the revision of this standard to improve implanted communications qualitatively.

UWB measurements can be performed from an experimental or a mathematical point of view. This thesis is mainly focused on the experimental assessment of the in-body UWB channel performance. In order to carry out this task, a comprehensive study of the UWB in-body channel has been performed. From the design of UWB antennas for this purpose to the study of the channel by using novel phantom-based measurement setups as well as in vivo measurements. In addition, diversity-based techniques has been evaluated in order to achieve more power on the receiver side so that the coverage between transmitter and receiver can be increased. 
The main contribution of this thesis are summed up in Section 6.1, whereas proposed future works are detailed in Section 6.2.

\subsection{Conclusions}

This thesis was focused on contributing to UWB channel characterization as well as enhancing the channel performance for future in-body communications.

In Chapter 3, achieving new implantable UWB transmitting antennas as well as on-body receivers was the main goal. Two optimization and miniaturization techniques were used for this purpose. On the one hand, the most popular method to optimize antennas to work inside the human body was applied. In this technique, firstly, the antenna is designed to work on frequencies that are above the target frequency band. Then, the antenna is wrapped with tissue layers. Taking into account the dielectric properties of human tissues, it is expected that the resonance frequency shifts down. Applying this technique, a new discone antenna was designed, miniaturized, optimized and manufactured for this purpose. In order to enlarge the bandwidth as well as to increase the input impedance, the back cavity technique and a short wall were added to the first design. In this way, a tiny antenna for the capsule endoscope was obtained achieving a bandwidth of $1 \mathrm{GHz}$ in the low part of the UWB frequency band, approximately. Same results were obtained after manufacturing the antenna with an aluminium material. Since the typical miniaturization procedure involves a large number of trials with certain success, a new approach to optimize and miniaturized UWB antennas was developed. In this case, the antenna was wrapped with the tissue layer since the first design stage. This means that the optimization and miniaturization procedures are applied considering the propagation medium from the beginning. In this way, the radiation parameters that the antennas have in free space were trying to keep up. With this technique, a well-known CPW-fed antenna with circular patch was miniaturized to work inside the body. As evidenced the obtained results, a broad bandwidth within UWB was achieved. Furthermore, the received power was high enough to establish a proper communication link despite the drop of the antenna efficiency. Besides, other radiation parameters such as the radiation pattern were kept as well. Moreover, the channel performance was similar compared with other larger antenna models for an IB2OB scenario and even better for certain distances between antennas in an IB2OFF scenario. Comparing both techniques, it can be concluded that the direct design procedure was more efficient than the traditional one. This is by the fact that the popular miniaturization procedure is based on a premise which does not seem to 
be good for antenna with large bandwidths. The optimization procedure can become ineffective and inefficient in design time (trial and error).

The direct design procedure was applied on other antennas for in-body communications. Specifically, a family of slot antennas was assessed as candidates of UWB on-body receivers. Two slot antennas were miniaturized and optimized by the direct antenna design procedure. The difference between both models was that two circles were added to optimize one of the slots. In both models were added a reflecting plane to focus the power to the area of interest. A broad bandwidth and good penetration were achieved with the slot antenna with circles. Besides, the input impedance was close to $50 \Omega$ and the losses were lower with this antenna within the lower part of UWB spectrum. After manufacturing the slot antenna with circles, the values of antenna matching obtained by simulations were compared with those obtained by using a human subject. The real values were below $-6 \mathrm{~dB}$ between 3 to $5.3 \mathrm{GHz}$, achieving an absolute bandwidth greater than $2 \mathrm{GHz}$ within the low part of UWB spectrum.

Chapter 4 deals with the experimental UWB channel characterization in two different promising scenarios. These scenarios were the IB2IB and the IB2OB. First of all, a measurement campaign in a phantom-based IB2IB scenario from 3.1 to $8.5 \mathrm{GHz}$ is carried out. Two identical UWB monopole antennas were used. Two monopole antennas miniaturized and optimized by the direct antenna procedure were used. In this analysis, it was noted that the signal significantly drops above $5.5 \mathrm{GHz}$. So only the contributions below this frequency should be considered since the values below the noise level must be disregarded. Afterwards, other measurement campaign was performed for the same scenario and antennas, but considering a lower bandwidth than in the previous study. The path loss models were fitted by a linear model. In this study of IB2IB channel performance, losses were compared when samples were considered different heights as well as considering all of them. As concluded, even though the slope was higher at the center heights and took an intermediate value considering all heights, there was no great variation. On the other hand, the diversity of the channel was assessed for the IB2IB scenario as well. With regard to the correlation in transmission, uncorrelated signals were achieved when the nodes were separated by $4 \lambda$ at least. In reception, the receivers had to be more than $2 \lambda$ away to reach uncorrelation.

In Chapter 4, the channel performance in several IB2OB scenarios was assessed as well. Firstly, the differences in the path loss values by using two different on-body receivers were analyzed. One was a slot patch antenna with a reflecting plane and the other one was a microstrip monopole antenna. As implanted antenna, the aforementioned UWB miniaturized antenna was used. 
The best performance was achieved with the slot patch antenna because the noise level was lower in comparison with the other one. This difference in the antenna performance was more remarkable as the frequency increases. With regard to the path loss using the slot patch antenna in the IB2OB phantombased scenario, it was better fitted by a linear approximation models in the lowest $500 \mathrm{MHz}$ subband $(3.1-3.6 \mathrm{GHz})$, but the trend changed to a logdistance model as the subband grows. Considering the whole available bandwidth $(3.1-5.1 \mathrm{GHz})$, the trend was slightly better fitted by a linear model. Moreover, the values obtained from an in vivo experiment were analyzed and discussed with those of a phantom-based setup. For that, the same antennas were used (slot and CPW-fed antennas). It was highly remarkable the good agreement between in vivo and phantom-based measurements. As the frequency increased, the measurements performed with the phantom reached the noise level, whereas the noise level was reached in higher frequency bands and further distances in the in vivo measurements. Unlike the phantom-based scenario, the path loss fitted better with a linear approximation model. This was due to the difference in measured points between one setup and another. However, there are not big differences either. Regarding the correlation in transmission for the phantom-based IB2OB scenario by using the slot antenna as on-body receiver and the UWB CPW monopole as in-body transmitter, the correlation increased as the distance between transmitters grew as well. Furthermore, the correlation in upper frequency subbands was higher than that of the low frequency ones. The results also evidence that the correlation values for the whole bandwidth were quite similar to those obtained at the lower subband. On the other hand, the correlation in reception was assessed for a phantom-based and in vivo scenarios. In both cases the correlation considerably decreased as the distance between receivers was higher. In the case of the phantom-based setup, the receivers should be separated more than $2 \lambda$ to obtain a low correlation. On the in vivo experiment, the correlation was too high when the transmitters are located in the same in-body position, and significantly decreased as the transmitting antennas are positioned in different in-body locations.

Chapter 5 was devoted to study methods based on diversity combining to enhance the channel performance and to compensate the high losses of the propagation channel. Time Reversal signal processing is applied in an IB2IB scenario. This scenario can be the the intermediate step in the connection between a deeply implanted node and an external one. The intermediate node can be considered as a relay. First of all, the reciprocity of the channel was studied and proved. Since the channel was reciprocal, it allows the use of Time Reversal technique. Using the Time Reversal technique, a gain between 4 and 
$11 \mathrm{~dB}$ was achieved. This enhancement implies that the distance between sensors could increase from 1 to $2 \mathrm{~cm}$, i.e. a 11-22\% of that without applying time reversal processing. This gain was higher when the correlation in transmission decreases and the received power was lower.

Finally, in the same chapter, Maximal-Ratio Combining technique is used in an IB2OB scenario for the same purpose. IB2OB communications are the most popular in the medical field. In order to reconstruct the channel impulse response, the CLEAN algorithm was applied. The gain in SNR applying MRC with three receivers varied from 4 to $12 \mathrm{~dB}$, which implied an increment of the distance between antennas of between 1 and $2 \mathrm{~cm}$. This increment led to an increment between $11-22 \%$ of that without using MRC technique, being higher when the distance between antennas increased.

\subsection{Perspective}

This thesis is a little grain of sand on a beach of thousands of miles long. The amount of future works that can be derived is countless. This thesis aims to be rigorous but general. This thesis attempts to cover all the phases until a realistic and accurate characterization of the in-body propagation channel is achieved.

Reducing the number of in vivo experiments is ethically and morally essential. Here, to solve this issue, a first approach of a high accurate phantombased setup is developed. Even though the validation with living tissues is in good agreement, new phantoms such as skin or fat should be manufactured and tested their effect on the propagation channel. In this way, the effects of the heterogeneity of the human tissues on channel characterization could be checked and compared with the results obtained from in vivo experiments as well as from the single phantom-based setup presented here.

As mentioned in this thesis, the literature is lack of UWB antennas for inbody communications. Since this is a harsh propagation medium, the involved antennas play an even more relevant role. The direct design procedure introduced in this thesis must be used to get new antenna models to test much more its effectiveness. The shape, size and technology of an antenna for in-body communications could depend on the medical application as well as the device in which the antenna will be embedded. Therefore, much smaller antenna models must be designed with this new approach for medical devices such as the endoscopic capsule. Besides, more sophisticated and complex models including certain parts of the capsule like the battery should be included in order to 
study its effect on radiation parameters. With regard to on-body receivers, the on-body antennas designed in this thesis and new derived models should be tested in more ex vivo experiments since the thickness of each human tissue is different according to the anatomy of the assessed human subject.

Since future wireless networks aim at interconnecting thousand and thousand of devices, more and more propagation scenarios must be studied. Regarding future communications with implantable nodes, this thesis tries to contribute to the gap that exists in literature. The in-body to in-body communication scenario is completely unexplored. Therefore, many more studies in more complex measurement setups must be carried out for later validation in an in vivo experiment. Something similar happens in the in-body to on-body scenario. Although the literature has contributions to this scenario within UWB bands, more studies should be performed considering experimental setups with high accurate phantoms in more complex environments. Furthermore, the experimental results should be checked with realistic simulations that take into account complex digital models to further corroboration of the findings.

At the same time that the measurement setup is enhanced and refined, other techniques based on channel diversity such as Equal Gain Combining or Selection Diversity could be tested. In the techniques used in this thesis, other kind of antennas can be chosen and the number of receivers can be increased to perform deeper studies. On the other hand, the transmission scheme could include a signal generator to reproduce a real situation. 


\section{Appendix A}

\section{Dielectric Properties of Human Body and Tissue-equivalent Phantoms}

There are two parameters that define the behavior of the electromagnetic fields: the permittivity $(\epsilon)$ which is related to the electric component, and the permeability $(\mu)$, related to the magnetic component of the field. These parameters describe how the electromagnetic waves travel through space. The propagation losses, reflections, speed, polarization and other effects are explained considering these properties from the materials involved in the propagation medium. The values of both parameters are usually given relative to those of vacuum as relative permittivity $\left(\epsilon_{r}\right)$ and relative permeability $\left(\mu_{r}\right)$. These are dimensionless, complex and also frequency dependent.

Normally, the propagation scenario is the air, where the waves reach the maximum speed and the minimum losses, and just the properties of the obstacles in between have to be taken into account. However, the number of unconventional scenarios is growing at this time, like in this thesis, where the body itself is the propagation medium. In the case of WBANs, the magnetic field can be neglected in the same way as in the air, because $\mu_{r}$ is close to 1 as well. Then, the relative permittivity becomes the key in such scenarios. 
As mentioned above, it is a frequency-dependent complex parameter, so it has a real and an imaginary part:

$$
\varepsilon(f)=\varepsilon_{r}^{\prime}-j \varepsilon_{r}^{\prime \prime}
$$

The real part is commonly known as dielectric constant, whereas the imaginary one is usually termed as loss factor. Sometimes, the loss factor is expressed as conductivity $(\mathrm{S} / \mathrm{m})$ :

$$
\sigma(f)=2 \pi f \varepsilon_{0} \varepsilon_{r}^{\prime \prime}
$$

As can be observed in the previous equation, the frequency determines the value of this property. There are plenty of studies about measuring the relative permittivity of the body tissues. When analyzing these properties with the frequency, it has been reported that there are some frequency ranges where they change abruptly, the so-called dispersion regions. This behavior can be observed in Figure A.1, where there are three changes in the slope.

These regions are related to the polarization mechanisms of the matter that forms the tissues. Each drop in the values of the dielectric constant takes place at certain frequency, when the mechanism is no longer present. The point where this phenomenon appears is called relaxation frequency. For instance, water molecules rotate following the electric field up to $19.3 \mathrm{GHz}$. From this frequency value, they cannot reach such rotation speed and start to mismatch and become out of phase. This misalignment is translated into losses, which

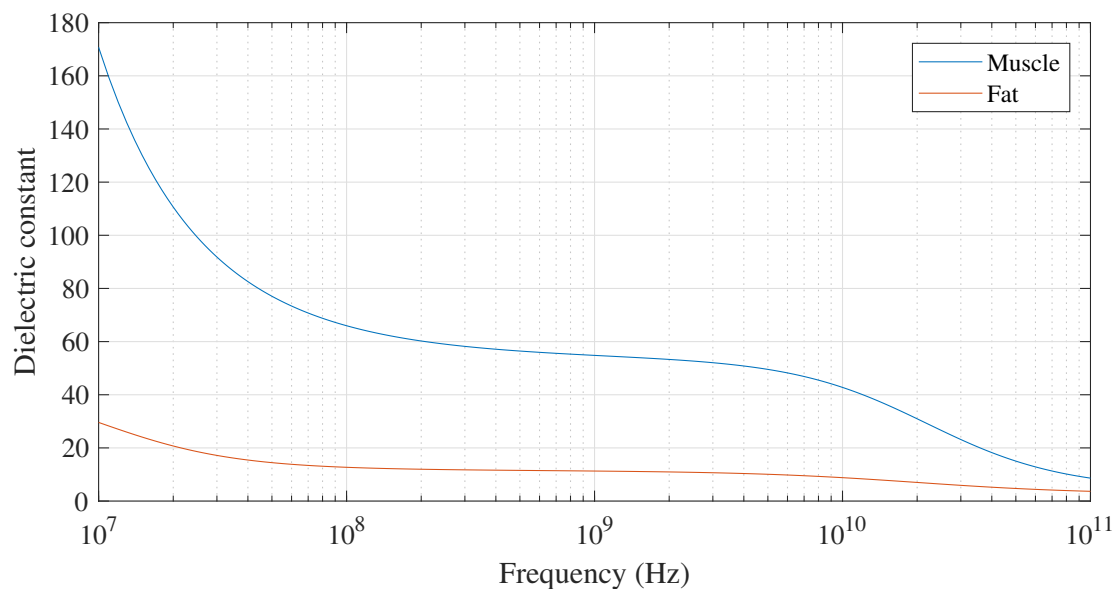

(a) 


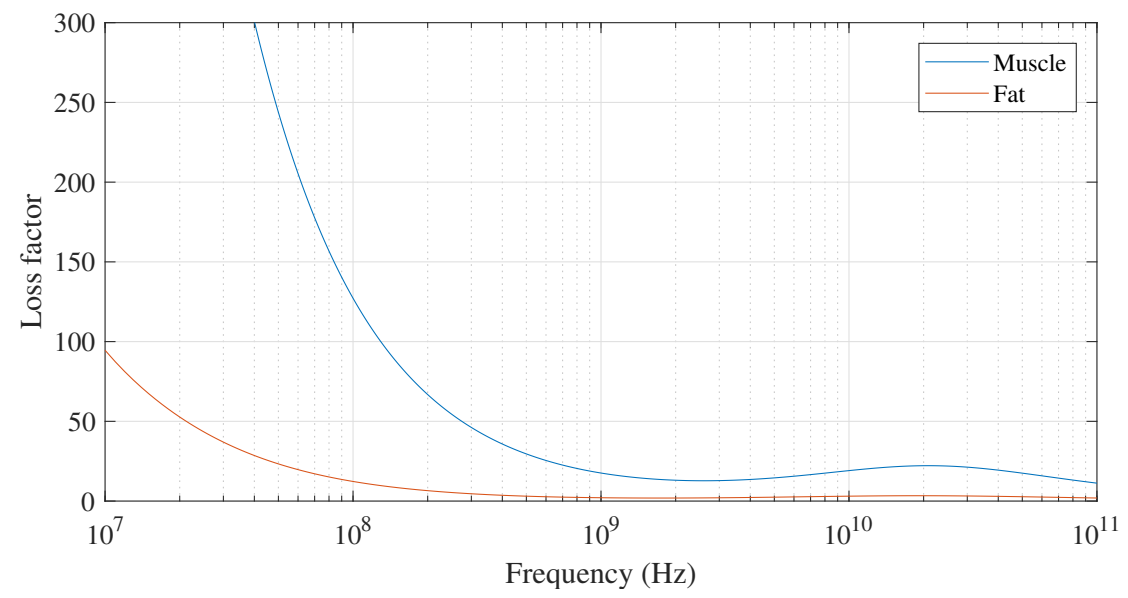

(b)

Figure A.1: Relative permittivity of the fat and muscle tissues according to [12]. Dielectric constant (a) and loss factor (b).

cause a peak in the loss factor at this frequency. The equation which best describes the behavior of the values of the relative permittivity is the ColeCole model:

$$
\varepsilon_{r}^{\prime}-j \varepsilon_{r}^{\prime \prime}=\varepsilon_{\infty}+\frac{\varepsilon_{s}-\varepsilon_{\infty}}{1+(j \omega \tau)^{1-\alpha}}+\frac{\sigma_{s}}{j \omega \varepsilon_{0}},
$$

where $\varepsilon_{\infty}$ is the infinite dielectric constant; $\varepsilon_{s}$ is the static dielectric constant; $\sigma_{s}$ is the conductivity; $\omega$ is the angular frequency; and $\tau$ is a time constant. This model achieves a good fitting for the dispersion regions by considering different poles, which can be selected from one to four, depending on the frequency range, i.e., the number of slope changes. Then, when measuring the values of the body tissues at certain frequencies, they are fitted to this model in a way that there is no need to provide all of the values but just the parameters of the model. In addition, the curves for the concerned tissue can be extrapolated for frequencies outside those measured. This can be useful when a frequency range cannot be reached with the technology of the moment.

The most cited work as a reference for the values of the body tissues is the Gabriel's compilation [12]. This source contains a database of 55 tissues, within a frequency range from $10 \mathrm{~Hz}$ up to $100 \mathrm{GHz}$. One can appreciate that most of the measurements were not performed on human beings, since this is restricted by ethical and legal reasons, but with different animals. Results for all of them 
are included and combined within the Cole-Cole model in order to achieve an average model for each tissue. In fact, there is a remarkable variability between the values of different specimens due to age, gender or weight, among other factors. This dispersion of the values is usually taken into account by establishing a tolerance region of around a 10\% deviation from the Gabriel values in order to match the tissue of interest. Temperature is other of the factors that changes the relative permittivity of the materials, and so that of the tissues. It can be assumed that the temperature that should be used in tests is that of the body in normal conditions, i.e., around 37 degrees Celsius. Thus, researchers try to measure the dielectric properties at this temperature regardless of the sample conditions, either in vivo or in vitro, where the excised tissue is no longer vascularized. This latest fact changes other properties such as the tissue moisture, which is extremely important in the final dielectric properties. Once excised, tissues begin to dry out, and water is an important constituent for most of them. There are studies that show the changes in the permittivity due to their hydration [98].

There are several methods and measurement techniques for characterizing the relative permittivity of the materials. Regarding the tissues, the openended coaxial technique is the preferred one because of its multiple advantages [99]-[101], including non-destructive and high bandwidth frequency measurements [102]. This method is basically comprised of an open-ended coaxial probe and a vector network analyzer, which measure the reflection parameter $\left(\mathrm{S}_{11}\right)$ of the interface between the material under test and the probe's tip. Then, the complex permittivity of the material can be calculated from the scattering parameter. For this, a calibration with at least three different wellknown materials need to be performed before, e.g., air, short circuit and water [103]. This calibration removes the influence of the elements of the setup such as the connectors, cables or the probe itself, in a way that at the end just the reflection on the tip surface is considered. The technique is suitable to measure liquids and semisolids, so it is convenient for most of the tissues.

These permittivity values are used afterwards as target when simulating a specific scenario in which the human body is present. Simulations are carried out with either computer or physical phantoms that match these reference values [104]. Regarding the physical or experimental phantoms, they are not trivial to obtain or ensure the matching with the target values. Replacing a tissue by a model made of a different material but with the same dielectric properties is challenging. It is not possible to replicate the same behavior in the whole frequency spectrum, so the attempts reported in the literature are limited to certain frequency bands [105]. Traditionally, these bands were nar- 
row because technologies at that time did not make use of large bandwidths. In these cases, a great deal of phantoms is reported in literature with their corresponding formulation [106]. Most of them achieve a remarkable approximation, imitating even decimal values. However, nowadays, the need of higher capacities has led to the demand of new technologies that benefit from wider bands, e.g., UWB (3.1-10.6 GHz). These kinds of phantoms are not easily found, since imitating not only the values but the behavior in frequency, i.e., the relaxation frequencies or dispersion regions, is restricted by the nature of the materials. There are many attempts trying to replicate the tissues along large frequency bands, being most of them unsuitable due to a bad approximation [40](see Figure A.2).

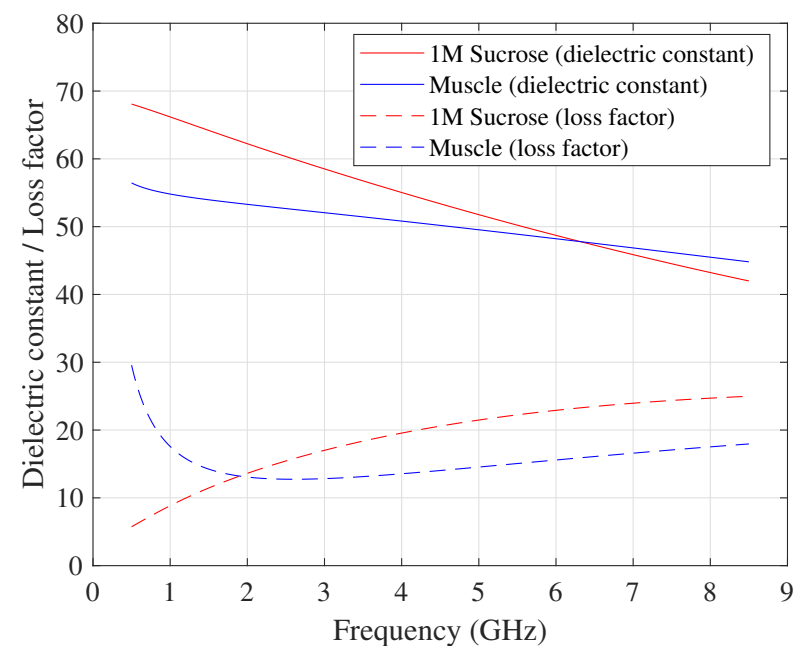

Figure A.2: Relative permittivity of the $1 \mathrm{M}$ sucrose solution proposed in [40] compared to the muscle tissue.

Recently, phantoms with high accuracy in such bandwidths has been reported in [107] (see Figure A.3). 


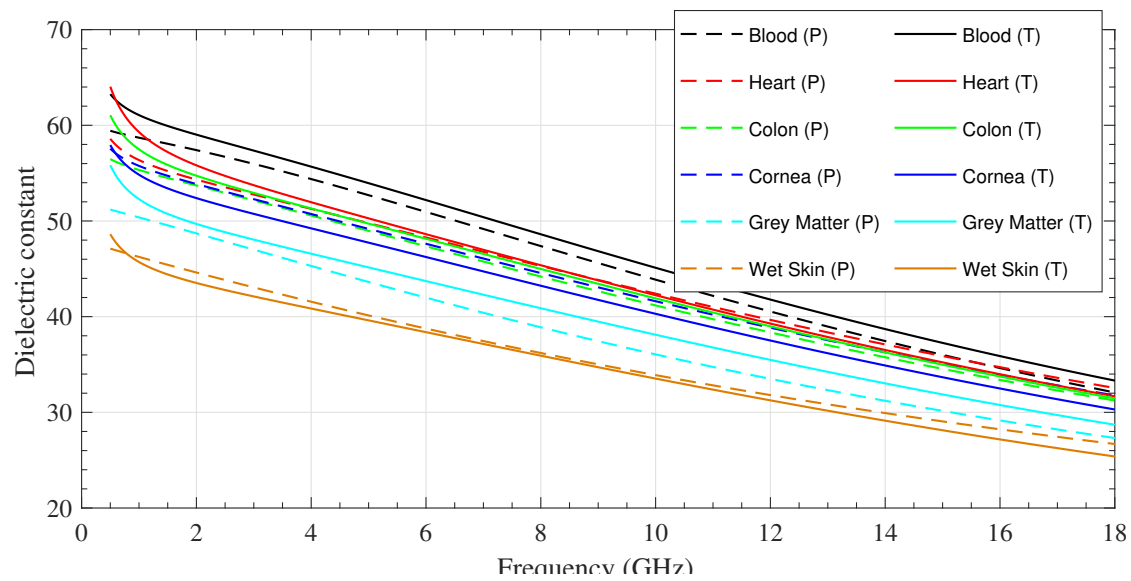

(a)

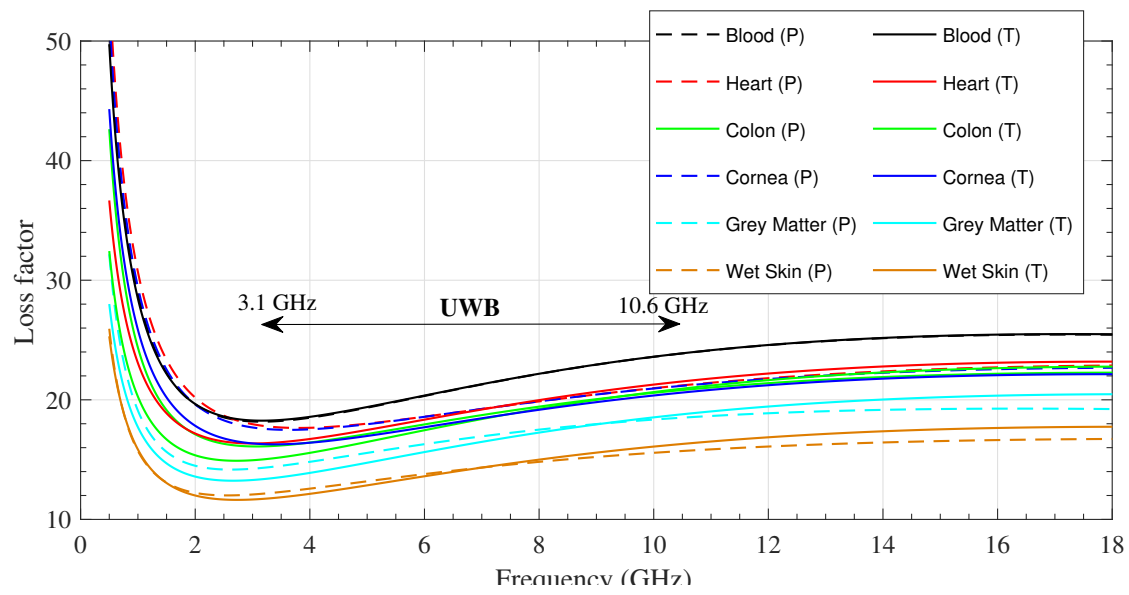

(b)

Figure A.3: Relative permittivity of several phantoms [54], [107] (P, dashed lines) in comparison with Gabriel's of their corresponding tissues ( $\mathrm{T}$, solid lines). Dielectric constant (a) and loss factor (b). 


\section{List of Figures}

1.1 Commercial capsule endoscopy. (a) Front and (b) side view. . . 3

1.2 Example of multiple nodes interconnected in a WBAN network

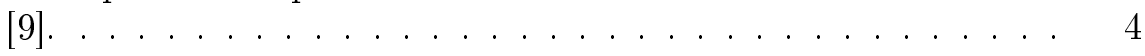

1.3 UWB frequency spectrum $[24] . \ldots \ldots \ldots \ldots$

2.1 Inner tissues of the CST Female Visible Human Model "Nelly" [53]. . . . . . . . . . . . . . . . . . 18

2.2 Experimental phantom-based measurement setup. . . . . . . 18

2.3 In vivo measurements. Antenna implanted in the abdominal region of a porcine subject. . . . . . . . . . . 19

2.4 Frequency domain channel sounding [53] . . . . . . . . . . 20

2.5 Measured relative permittivity of the UWB phantom compared to the values of human muscle tissue reported in [12] . . . . 22

2.6 Keysight Vector Network Analyzer. . . . . . . . . . . . . . 23

2.7 Tracking system. (a) Mid-range transmitter, (b) sensor, (c) Desktop electronics unit [55]. . . . . . . . . . . . . . 24

2.8 XYZ linear positioners [56]. . . . . . . . . . . . . 25 
2.9 Step motors and coils $[56] \ldots \ldots \ldots \ldots$. . . . . . . . 26

2.10 IB2IB phantom-based experimental measurement setup $\ldots . .27$

2.11 IB2OB phantom-based experimental measurement setup . . . . 28

2.12 Custom program interface for phantom-based measurement campaigns. . . . . . . . . . . . . . . . . . . 29

2.13 Algorithm of Grid Points movement. . . . . . . . . . . . . 31

2.14 Pig anatomy $[58] \ldots \ldots \ldots \ldots \ldots$

2.15 In-body antenna placed among the guts of the small intestine. Picture taken by the laparoscopic camera. . . . . . . . . . . 34

2.16 Locations of the on-body receiver over the pig's belly. . . . . . . 35

2.17 On-body receiver placed on the belly and covered by the isolator layer. . . . . . . . . . . . . . . . . 35

2.18 Custom software interface for in vivo measurement campaigns. $\quad 36$

2.19 Algorithm of the software for in vivo measurements. . . . . . . 37

3.1 Scheme of antenna structure [61] . . . . . . . . . . . . . 41

3.2 Types of radiation pattern [61]. . . . . . . . . . . . 43

3.3 UWB implantable antennas. (a) For capsule endoscope [33]. (b) Loop antenna to be implanted into the brain [62]. (c) Flexible implantable antenna [63]. . . . . . . . . . . . . 45

3.4 Manufactured UWB On-Body antenna model [66] . . . . . . . 46

3.5 Wireless capsule endoscopy structure [33]. . . . . . . . . . . . 49

3.6 Discone antenna parameters. . . . . . . . . . . . 50

3.7 Discone antenna model in free space. . . . . . . . . . . . 52

3.8 Reflection coefficient of discone antenna designed to work at high frequencies (Free space). . . . . . . . . . . 52

3.9 Real part of the input impedance of discone antenna (Free space). 53 
3.10 Imaginary part of the input impedance of discone antenna (Free

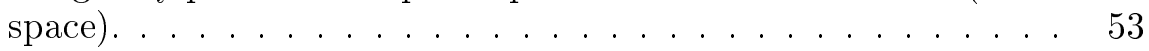

3.11 Discone antenna embedded into the capsule endoscope. . . . . . 54

3.12 Capsule endoscope and antenna wrapped with the phantom layer. 55

$3.13 \mathrm{XY}$ cross section of the discone antenna and capsule. . . . . . 55

3.14 Reflection coefficient of the embedded antenna into the capsule endoscope and wrapped with the phantom layer. . . . . . 5 56

3.15 Discone antenna model with 15 steps. . . . . . . . . . 57

3.16 Discone antenna filled with ferrite. . . . . . . . . 57

3.17 Discone antenna optimized by back cavity technique . . . . . 58

3.18 Reflection coefficient of the discone antenna optimized by back cavity technique . . . . . . . . . . . . . 59

3.19 Real part of the input impedance of the discone antenna optimized by back cavity technique . . . . . . . . . . . 59

3.20 Imaginary part of the input impedance of the discone antenna optimized by back cavity technique . . . . . . . . 60

3.21 Discone antenna optimized by back cavity technique and short wall . . . . . . . . . . . . . . . . 61

3.22 Reflection coefficient of the discone antenna optimized by back cavity technique and short wall . . . . . . . . . . 62

3.23 Real part of the input impedance of the discone antenna optimized by back cavity technique and short wall . . . . . . . .

3.24 Imaginary part of the input impedance of the discone antenna optimized by back cavity technique and short wall . . . . . .

3.25 Discone antenna optimized by back cavity technique and short wall including a copper layer of $1 \mathrm{~mm}$ of thickness. . . . . . . 64

3.26 Reflection coefficient of the discone antenna optimized by back cavity technique and short wall including a copper layer of 1 mm of thickness . . . . . . . . . . . . . . 
3.27 Real part of the input impedance of the discone antenna optimized by back cavity technique and short wall including a copper layer of $1 \mathrm{~mm}$ of thickness . . . . . . . . . . . . . . . 65

3.28 Imaginary part of the input impedance of the discone antenna optimized by back cavity technique and short wall including a copper layer of $1 \mathrm{~mm}$ of thickness . . . . . . . . . . . . 65

3.29 Manufactured discone antennas. . . . . . . . . . . . . 66

3.30 (a) Dielectric constant and (b) loss factor values of the phantom described in [40] and the real values of the human muscle tissue

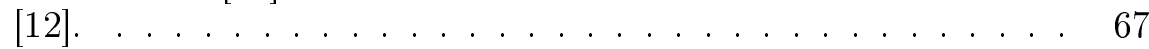

3.31 Discone antenna embedded into a polyethylene capsule. . . . . 67

3.32 Reflection coefficient of manufactured antenna . . . . . . . . 68

3.33 Real and Imaginary part of the input impedance of the manufactured discone antenna . . . . . . . . . . . . . . 68

3.34 Most extended procedure for antenna optimization and miniaturization .................... 69

3.35 New approach of miniaturization and optimization. Direct antenna design technique. . . . . . . . . . . . . . . . 70

3.36 Candidate antenna wrapped with phantom layer. . . . . . . . . 70

3.37 UWB optimized planar antenna dimensions. . . . . . . . . . . . 71

3.38 Gain pattern of the miniaturized antenna in the human muscle tissue at 3.1, 4.1, 5.1 and $6.1 \mathrm{GHz}$. . . . . . . . . . . . . 72

3.39 Manufactured UWB antenna which is miniaturized and optimized by the direct antenna design procedure. . . . . . . . . 73

3.40 Measured and simulated return loss from 3.1 to $8.5 \mathrm{GHz}$. . . . 74

3.41 Real part of the input impedance of the miniaturized antenna. . 75

3.42 Imaginary part of the input impedance of the miniaturized antenna. . . . . . . . . . . . . . . . . . . . 75

3.43 Transmitting and receiving UWB antennas. . . . . . . . . . 76 
3.44 Measurement setups for each scenario. . . . . . . . . . 76

3.45 Relative received power as a function of the frequency in the IB2OB scenario for different values of $d=d_{\text {in }}$ when (a) the large and (b) the miniaturized antennas acted as transmitters. . 79

3.46 Relative received power as a function of the frequency in the IB2OFF scenario for different values of $d=d_{\text {off }}$ when (a) the large and (b) the miniaturized antennas acted as transmitters. . 81

3.47 Multilayer Antenna Model for the CST simulations. . . . . . . . 82

3.48 Fork-shaped microstrip feeding line. . . . . . . . . . . 83

3.49 Proposed slotted patch structures. . . . . . . . . . . . 84

3.50 Side view of the proposed antenna structure. . . . . . . 85

3.51 Simulated reflection coefficient for the slotted patch structures within UWB. . . . . . . . . . . . . . . 86

3.52 Real part of complex impedance for each slot patch structure. . 86

3.53 Imaginary part of complex impedance for each slot patch structure. 87

3.54 Near radiated E-Field $(\mathrm{dBV} / \mathrm{m})$ in the human tissues. . . . . . 88

$\begin{array}{ll}3.55 \text { Manufactured prototype of the slot patch antenna with circles. } & 89\end{array}$

3.56 Measurement setup. . . . . . . . . . . . . . . . . . 89

3.57 Simulated and measured reflection coefficient of the manufactured antenna. . . . . . . . . . . . . 90

3.58 Reflection coefficient of the slotted patch antenna over the pig's belly. . . . . . . . . . . . . . . . 90

4.1 UWB in-body antennas used in the IB2IB experimental scenario 95

4.2 Frequency transfer function $H(f)$ in several in-body locations. . 96

4.3 Measured reflection coefficients from 3.1 to $5.1 \mathrm{GHz}$ where both antennas are submerged into the liquid phantom. . . . . . . 97 
4.4 IB2IB experimental measurement setup. Locations of receiving antenna. ................... 98

4.5 Path loss as a function of distance between antennas considering RX1. Values and models for different heights (a) and all the measured points (b). ................ 100

4.6 Scheme for the assessment of the correlation in transmission in the IB2IB scenario . . . . . . . . . . . . . . . 101

4.7 Correlation coefficients at different heights in the experimental IB2IB scenario using RX1. $\mathrm{Z}=-2 \Delta \mathrm{z}(\mathrm{a}), \mathrm{Z}=-\Delta \mathrm{z}$ (b) and $\mathrm{Z}=$ 0 (c) $\mathrm{Z}=-\Delta \mathrm{z}(\mathrm{d})$ and $\mathrm{Z}=2 \Delta \mathrm{z}(\mathrm{e})$. . . . . . . . . . . . . 102

4.8 Complementary cumulative distribution function (CCDF) for correlation coefficients in reception. . . . . . . . . . . 104

4.9 IB2OB measurement setup. Grid of measurement points. . . . . 106

4.10 Path loss values per subband (a-d) and the whole available bandwidth (e) using two on-body receivers particularized for the IB2OB scenario. . . . . . . . . . . . . . . . . . 108

4.11 Location of the implanted antenna in the in vivo experiment . . 111

4.12 Path loss values per subband and the whole available bandwidth for in vivo (black points) and phantom-based (red points) setups particularized for the IB2OB scenario. . . . . . . . . . . . . . . 112

4.13 Measuring points considered in each half for the calculation of correlation coefficients in transmission for the phantom-based measurements . . . . . . . . . . . . . . . 115

4.14 Power delay profile for 5 different depths in the $\mathrm{X}$ axis considering RX1..................... 116

4.15 Locations of on-body antenna to study the correlation in reception in the IB2OB phantom-based scenario . . . . . . . . . . . . 117

4.16 Complementary cumulative distribution function (CCDF) for correlation coefficients in reception. . . . . . . . . . . . 118

5.1 Scheme of TR technique for a multi-receiver system . . . . . . 123 
5.2 Architecture of a receiver using MRC technique . . . . . . . . 124

5.3 CLEAN algorithm . . . . . . . . . . . . 126

5.4 Forward transmission coefficient in both directions (from port 1 to port 2 and viceversa) in three transmitting antenna locations at different $\mathrm{Z}$ planes. $\mathrm{Z}=-\Delta \mathrm{z}(\mathrm{a}), \mathrm{Z}=0$ (b) and $\mathrm{Z}=\Delta \mathrm{z}(\mathrm{c}) . \quad 129$

5.5 Peak to peak gain at different $\mathrm{Z}$ planes . . . . . . . . . . 130

5.6 Transmitted signal in frequency (a) and time (b) domain . . . . 132

5.7 Original and reconstructed received signal using the CLEAN algorithm with a threshold of (a) $10 \mathrm{~dB}$ and (b) $20 \mathrm{~dB} \ldots \ldots 133$

5.8 Gain in SNR applying MRC at different heights. (a) $\mathrm{Z}=0$, (b) $\mathrm{Z}=\Delta z$, (b) $\mathrm{Z}=2 \Delta z \ldots \ldots \ldots \ldots \ldots \ldots$

A.1 Relative permittivity of the fat and muscle tissues according to [12]. Dielectric constant (a) and loss factor (b). . . . . . . 145

A.2 Relative permittivity of the $1 \mathrm{M}$ sucrose solution proposed in [40] compared to the muscle tissue. . . . . . . . . . . . . 147

A.3 Relative permittivity of several phantoms [54], [107] (P, dashed lines) in comparison with Gabriel's of their corresponding tissues (T, solid lines). Dielectric constant (a) and loss factor (b). . . 148 



\section{List of Tables}

3.1 Dimensions of fork-shaped feeding line. . . . . . . . . . 83

3.2 Dimensions of slot models. . . . . . . . . . . . . . . . 84

4.1 Configuration of the VNA and the positioner. Preliminary analysis in the IB2IB scenario. . . . . . . . . . . . . . . 95

4.2 Configuration of the VNA and the positioner. IB2IB channel

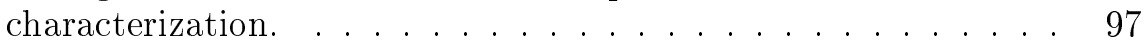

4.3 Fitting parameters of the approximation model for the IB2IB scenario. . . . . . . . . . . . . . . . 100

4.4 Configuration of the VNA and the positioner. IB2OB channel characterization. . . . . . . . . . . . . . 105

4.5 Fitting parameters of the approximation models. Monopole antenna. . . . . . . . . . . . . . 107

4.6 Fitting parameters of the approximation models. Slotted patch antenna. . . . . . . . . . . . . . . 107

4.7 Fitting parameters of the approximation models for the in vivo measurements . . . . . . . . . . . . . . . 113

4.8 Probability for correlation coefficients $\geq 0.8 \ldots \ldots \ldots$ 
4.9 Correlation coefficients when the in-body antennas are located in the same location . . . . . . . . . . . . . . 119

4.10 Correlation coefficients when the in-body antennas are located in different location . . . . . . . . . . . . . . . . . . . . 119

5.1 Probability that correlation between $S_{21}$ and $S_{12}$ is greater than 0.8. . . . . . . . . . . . . . . . . 128

5.2 Correlation values for different thresholds . . . . . . . . . 133 


\section{Glossary}

\section{BER}

Bit Error Rate.

\section{CCDF}

Complementary Cumulative Distribution Function.

\section{CPW}

Coplanar Waveguide.

\section{CST}

Computer Simulation Technology.

CT

Computed Tomography scan.

\section{$\mathrm{dBm}$}

decibel-milliwatts.

\section{FCC}

Federal Communications Commission. 


\section{GHz}

Gigahertz.

\section{HFSS}

High Frequency Electromagnetic Field Simulation.

\section{HULAFE}

Hospital Universitari i Politècnic La Fe.

\section{IB2IB}

In-Body to In-Body.

\section{IB2OB}

In-Body to On-Body.

\section{IB2OFF}

In-Body to Off-Body.

\section{IDFT}

Inverse Discrete Fourier Transform.

\section{IFFT}

Inverse Fast Fourier Transform.

\section{ISM}

Industrial, Scientific and Medical.

\section{$\mathrm{kHz}$}

kilohertz.

\section{LOS}

Line-Of-Sight. 


\section{MICS}

Medical Implant Communication System.

\section{MPCs}

Multipath Components.

\section{MRC}

Maximal-Ratio Combining.

\section{MRI}

Magnetic Resonance Imaging.

\section{PEC}

Perfect Electrical Conductor.

\section{PP}

Polypropylene.

SF

Shortening Factor.

SNR

Signal-to-Noise ratio.

TR

Time Reversal.

\section{UPV}

Universitat Politècnica de València.

\section{UWB}

Ultra-Wide band. 
VB

Visual Basic.

VNA

Vector Network Analyzer.

\section{WBAN}

Wireless Body Area Network.

\section{WCE}

Wireless Capsule Endoscopy. 


\section{Bibliography}

[1] A. Guy, "History of Biological Effects and Medical Applications of Microwave Energy", IEEE Transactions on Microwave Theory and Techniques, vol. 32, no. 9, pp. 1182-1200, Sep. 1984, ISSN: 0018-9480. DOI: 10.1109/TMTT. 1984.1132830.

[2] H. L. Abrams and B. J. McNeil, "Medical Implications of Computed Tomography (Cat Scanning)", New England Journal of Medicine, vol. 298, no. 5, pp. 255-261, Feb. 1978, ISSN: 0028-4793.

[3] M. A. Shampo and R. A. Kyle, "Karl Theodore Dussik, Pioneer in Ultrasound", Mayo Clinic Proceedings, vol. 70, no. 12, p. 1136, Dec. 1995, ISSN: 00256196. DOI: 10.4065/70.12.1136.

[4] G. Iddan, G. Meron, A. Glukhovsky, and P. Swain, "Wireless capsule endoscopy.", Nature, vol. 405, no. 6785, pp. 417-418, May 2000, ISSN: 0028-0836. DOI: $10.1038 / 35013140$.

[5] Z Fireman, E Mahajna, E Broide, M Shapiro, L Fich, A Sternberg, Y Kopelman, and E Scapa, "Diagnosing small bowel Crohn's disease with wireless capsule endoscopy.", Gut, vol. 52, no. 3, pp. 390-2, Mar. 2003, ISSN: 0017-5749. DOI: 10.1136/GUT .52.3.390.

[6] H. Burri and D. Senouf, "Remote monitoring and follow-up of pacemakers and implantable cardioverter defibrillators.", Europace : European 
pacing, arrhythmias, and cardiac electrophysiology : journal of the working groups on cardiac pacing, arrhythmias, and cardiac cellular electrophysiology of the European Society of Cardiology, vol. 11, no. 6, pp. 7019, Jun. 2009, ISSN: 1532-2092. DOI: 10.1093/europace/eup110.

[7] R. Chávez-Santiago, K. Sayrafian-Pour, A. Khaleghi, K. Takizawa, J. Wang, I. Balasingham, and H.-B. Li, "Propagation models for IEEE 802.15.6 standardization of implant communication in body area networks", IEEE Communications Magazine, vol. 51, no. 8, pp. 80-87, Aug. 2013, ISSN: 0163-6804. DOI: 10.1109/MCOM.2013.6576343.

[8] IEEE Standards Association, 802.15.6-2012 - IEEE Standard for Local and metropolitan area networks - Part 15.6: Wireless Body Area Networks. Institute of Electrical and Electronics Engineers, 2012, ISBN: 9780738172064 .

[9] Wireless Body Area Network, http: //www.techsparks .co.in/thesisin-wireless-body-area-network, Accessed: 2018-04-17.

[10] T. S. Rappaport, Wireless communications : principles and practice. Dorling Kindersley, 2009, p. 707, ISBN: 813172882X.

[11] W. G. Scanlon, J. B. Burns, and N. E. Evans, "Radiowave propagation from a tissue-implanted source at $418 \mathrm{MHz}$ and $916.5 \mathrm{MHz}$. ", IEEE transactions on bio-medical engineering, vol. 47, no. 4, pp. 527-34, Apr. 2000, ISSN: 0018-9294. DOI: 10.1109/10.828152.

[12] C. Gabriel, "Compilation of the Dielectric Properties of Body Tissues at RF and Microwave Frequencies", Texas (USA), Tech. Rep., Jun. 1996, p. 273. DOI: ReportN.AL/OE-TR-1996-0037.

[13] R. Chávez-Santiago, C. Garcia-Pardo, A. Fornes-Leal, A. Vallés-Lluch, G. Vermeeren, W. Joseph, I. Balasingham, and N. Cardona, "Experimental Path Loss Models for In-Body Communications Within 2.36$2.5 \mathrm{GHz}$ ", IEEE Journal of Biomedical and Health Informatics, vol. 19, no. 3, pp. 930-937, May 2015, ISSN: 21682194. DOI: 10 . 1109 / JBHI . 2015.2418757.

[14] D. Kurup, M. Scarpello, G. Vermeeren, W. Joseph, K. Dhaenens, F. Axisa, L. Martens, D. Vande Ginste, H. Rogier, and J. Vanfleteren, 
"In-body path loss models for implants in heterogeneous human tissues using implantable slot dipole conformal flexible antennas", EURASIP Journal on Wireless Communications and Networking, vol. 2011, no. 1, p. 51, Dec. 2011, ISSN: 1687-1499. DOI: 10.1186/1687-1499-2011-51.

[15] D. Kurup, W. Joseph, G. Vermeeren, and L. Martens, "In-body path loss model for homogeneous human tissues", IEEE Transactions on Electromagnetic Compatibility, vol. 54, no. 3, pp. 556-564, 2012, ISSN: 00189375. DOI: $10.1109 /$ TEMC .2011.2164803.

[16] S. Ashok Kumar and T. Shanmuganantham, "Design and Analysis of an Implantable CPW-Fed X-Monopole Antenna for 2.45-GHz ISM Band Applications", International Journal of Microwave and Wireless Technologies, vol. 20, no. 3, pp. 246-252, Mar. 2014, ISSN: 1530-5627. DOI: $10.1089 / \mathrm{tmj} .2013 .0186$.

[17] J. Gemio, J. Parron, and J. Soler, "Human Body Effects on implantable antenna for ISM bands applications: Models comparison and propagation losses study", Progress In Electromagnetics Research, vol. 110, pp. 437-452, 2010, ISSN: 1559-8985. DOI: $10.2528 /$ PIER10102604.

[18] P. Patel, M. Sarkar, and S. Nagaraj, "Ultra wideband channel characterization for invasive biomedical applications", in 2016 IEEE 17th Annual Wireless and Microwave Technology Conference (WAMICON), Apr. 2016, pp. 1-6, ISBN: 978-1-5090-1199-5. DOI: 10.1109/WAMICON . 2016.7483835.

[19] A. Molisch, "Ultrawideband Propagation Channels-Theory, Measurement, and Modeling", IEEE Transactions on Vehicular Technology, vol. 54, no. 5, pp. 1528-1545, Sep. 2005, ISSN: 0018-9545. DOI: $10.1109 /$ TVT . 2005.856194.

[20] European Commission, "2007/131/EC: Commission Decision of 21 February 2007 on allowing the use of the radio spectrum for equipment using ultra-wideband technology in a harmonised manner in the Community", Tech. Rep., 2007, pp. 33-36.

[21] A. F. Molisch, "Ultra-wide-band propagation channels", Proceedings of the IEEE, vol. 97, no. 2, pp. 353-371, Feb. 2009, ISSN: 0018-9219. DOI: 10.1109/JPROC . 2008. 2008836. 
[22] European Telecommunications Standards Institute, "ETSI EN 302065. Short Range Devices (SRD) using Ultra Wide Band technology (UWB); Harmonised Standard covering the essential requirements of article 3.2 of the Directive 2014/53/EU", Tech. Rep., 2016, p. 32.

[23] X. Shen, M. Guizani, R. Caiming Qiu, and T. Le-Ngoc, Eds., UltraWideband Wireless Communications and Networks. John Wiley \& Sons, 2007, p. 322, ISBN: 0470028513.

[24] UWB frequency spectrum, https://www.eliko.ee/uwb-technologyindoor-positioning/, Accessed: 2018-04-17.

[25] A. Ghildiyal, K. Amara, R. D. Molin, B. Godara, A. Amara, and R. K. Shevgaonkar, "UWB for in-body medical implants: A viable option", in 2010 IEEE International Conference on Ultra-Wideband, Nanjing: IEEE, Sep. 2010, pp. 1-4, ISBN: 978-1-4244-5305-4. DOI: 10.1109 / ICUWB . 2010.5615732.

[26] R. Chávez-Santiago, I. Balasingham, and J. Bergsland, "Ultrawideband Technology in Medicine: A Survey", Journal of Electrical and Computer Engineering, vol. 2012, pp. 1-9, 2012, ISSN: 20900147. DOI: 10.1155/ 2012/716973.

[27] E. Chow, M. Morris, and P. Irazoqui, "Implantable RF Medical Devices: The Benefits of High-Speed Communication and Much Greater Communication Distances in Biomedical Applications", IEEE Microwave Magazine, vol. 14, no. 4, pp. 64-73, Jun. 2013, ISSN: 1527-3342. DOI: 10.1109/MMM. 2013.2248586.

[28] R. Chavez-Santiago and I. Balasingham, "Ultrawideband Signals in Medicine [Life Sciences]", IEEE Signal Processing Magazine, vol. 31, no. 6, pp. 130136, Nov. 2014, ISSN: 1053-5888. DOI: 10.1109/MSP. 2014.2340234.

[29] M. R. Yuce and T. Dissanayake, "Easy-to-swallow wireless telemetry", IEEE Microwave Magazine, vol. 13, no. 6, pp. 90-101, 2012, ISSN: 15273342. DOI: $10.1109 /$ MMM.2012.2205833.

[30] Wee Chang Khor and M. E. Bialkowski, "Investigations into an uwb microwave radar system for breast cancer detection", in 2007 IEEE Antennas and Propagation International Symposium, IEEE, Jun. 2007, 
pp. 2160-2163, ISBN: 978-1-4244-0877-1. DOI: 10 . 1109 / APS . 2007 . 4395955.

[31] X. Xiao and T. Kikkawa, "Early Breast Cancer Detection by Ultrawide Band Imaging with Dispersion Consideration", Japanese Journal of Applied Physics, vol. 47, no. 4, pp. 3209-3213, Apr. 2008, ISSN: 0021-4922. DOI: $10.1143 /$ JJAP . 47 . 3209.

[32] A. Fornes-Leal, C. Garcia-Pardo, M. Frasson, V. Pons Beltrán, and N. Cardona, "Dielectric characterization of healthy and malignant colon tissues in the $0.5-18 \mathrm{GHz}$ frequency band", Physics in Medicine and Biology, vol. 61, no. 20, pp. 7334-7346, Oct. 2016. DOI: 10.1088/00319155/61/20/7334.

[33] Q. Wang, K. Wolf, and D. Plettemeier, "An UWB capsule endoscope antenna design for biomedical communications", in 2010 3rd International Symposium on Applied Sciences in Biomedical and Communication Technologies (ISABEL 2010), Rome: IEEE, Nov. 2010, pp. 1-6, ISBN: 978-1-4244-8131-6. DOI: 10.1109/ISABEL . 2010.5702832.

[34] M. R. Yuce, T. Dissanayake, and H. C. Keong, "Wireless telemetry for electronic pill technology", in 2009 IEEE Sensors, Oct. 2009, pp. 14331438. DOI: $10.1109 /$ ICSENS . 2009.5398440.

[35] B. Moussakhani, R. Chavez-Santiago, and I. Balasingham, "Multi model tracking for localization in wireless capsule endoscopes", in Proceedings of the 4th International Symposium on Applied Sciences in Biomedical and Communication Technologies, New York, USA: ACM Press, Oct. 2011, pp. 1-5, ISBN: 9781450309134. DOI: 10.1145/2093698.2093857.

[36] R. Chávez-Santiago, J. Wang, and I. Balasingham, "The ultra wideband capsule endoscope", in 2013 IEEE International Conference on UltraWideband (ICUWB), 2013, pp. 72-78. DOI: 10 . 1109 / ICUWB . 2013. 6663825.

[37] A. Khaleghi, R. Chávez-Santiago, and I. Balasingham, "An improved ultra wideband channel model including the frequency-dependent attenuation for in-body communications", in Proceedings of the Annual International Conference of the IEEE Engineering in Medicine and Bi- 
ology Society, EMBS, San Diego, CA, Sep. 2012, pp. 1631-1634, ISBN: 9781424441198. DOI: 10.1109/EMBC . 2012.6346258.

[38] C. Andreu, C. Garcia-Pardo, A. Fomes-Leal, M. Cabedo-Fabrés, and N. Cardona, "Uwb in-body channel performance by using a direct antenna designing procedure", in 2017 11th European Conference on Antennas and Propagation (EUCAP), 2017, pp. 278-282. DOI: 10.23919/EuCAP. 2017.7928416.

[39] T. Takimoto, T. Onishi, K. Saito, M. Takahashi, S. Uebayashi, and K. Ito, "Characteristics of biological tissue equivalent phantoms applied to UWB communications", Electronics and Communications in Japan, Part I: Communications (English translation of Denshi Tsushin Gakkai Ronbunshi), vol. 90, no. 5, pp. 48-55, May 2007, ISSN: 87566621. DOI: 10.1002/ecja. 20300.

[40] H. Yamamoto, J. Zhou, and T. Kobayashi, "Ultra wideband electromagnetic phantoms for antennas and propagation studies", IEICE Transactions on Fundamentals of Electronics, Communications and Computer Sciences, vol. E91-A, no. 11, pp. 3173-3182, Nov. 2008, ISSN: 09168508. DOI: $10.1093 /$ ietfec/e91-a.11.3173.

[41] C. Andreu, S. Castelló-Palacios, C. Garcia-Pardo, A. Fornes-Leal, A. Vallés-Lluch, and N. Cardona, "Spatial In-Body Channel Characterization Using an Accurate UWB Phantom", IEEE Transactions on $\mathrm{Mi}$ crowave Theory and Techniques, vol. 64, no. 11, pp. 3995-4002, Nov. 2016, ISSN: 0018-9480. DOI: 10.1109/TMTT .2016. 2609409.

[42] R. Chávez-Santiago, C. García-Pardo, A. Fornes-Leal, A. Vallés-Lluch, I. Balasingham, and N. Cardona, "Ultra wideband propagation for future in-body sensor networks", in 2014 IEEE 25th Annual International Symposium on Personal, Indoor, and Mobile Radio Communication (PIMRC), Sep. 2014, pp. 2160-2163. DOI: 10.1109/PIMRC . 2014. 7136530.

[43] C. Andreu, C. Garcia-Pardo, S. Castelló-Palacios, A. Vallés-Lluch, and N. Cardona, "Frequency dependence of uwb in-body radio channel characteristics", IEEE Microwave and Wireless Components Letters, vol. 28, no. 4, pp. 359-361, 2018, ISSN: 1531-1309. DOI: 10.1109/LMWC . 2018. 2808427. 
[44] M. J. Association of American Medical Colleges., Academic medicine : journal of the Association of American Medical Colleges. 6. Hanley \& Belfus, Jun. 1989, vol. 74, pp. 667-670.

[45] A. Christ, W. Kainz, E. G. Hahn, K. Honegger, M. Zefferer, E. Neufeld, W. Rascher, R. Janka, W. Bautz, J. Chen, B. Kiefer, P. Schmitt, H. P. Hollenbach, J. Shen, M. Oberle, D. Szczerba, A. Kam, J. W. Guag, and N. Kuster, "The Virtual Family development of surface-based anatomical models of two adults and two children for dosimetric simulations", Physics in Medicine and Biology, vol. 55, no. 2, pp. 23-38, Jan. 2010, ISSN: 0031-9155. DOI: 10.1088/0031-9155/55/2/N01.

[46] M. C. Gosselin, E. Neufeld, H. Moser, E. Huber, S. Farcito, L. Gerber, M. Jedensjo, I. Hilber, F. D. Gennaro, B. Lloyd, E. Cherubini, D. Szczerba, W. Kainz, and N. Kuster, "Development of a new generation of high resolution anatomical models for medical device evaluation. The Virtual Population 3.0", Physics in Medicine and Biology, vol. 59, no. 18 , pp. 5287-5303, Sep. 2014, ISSN: 0031-9155. DOI: 10.1088/0031$9155 / 59 / 18 / 5287$.

[47] D. Anzai, K. Katsu, R. Chávez-Santiago, Q. Wang, D. Plettemeier, J. Wang, and I. Balasingham, "Experimental Evaluation of Implant UWB-IR Transmission With Living Animal for Body Area Networks", IEEE Transactions on Microwave Theory and Techniques, vol. 62, no. 1, pp. 183-192, Jan. 2014, ISSN: 00189480. DOI: 10 . 1109/TMTT . 2013. 2291542.

[48] M. Lazebnik, E. L. Madsen, G. R. Frank, and S. C. Hagness, "Tissuemimicking phantom materials for narrowband and ultrawideband microwave applications", Physics in Medicine and Biology, vol. 50, no. 18, pp. 4245-4258, Sep. 2005, ISSN: 0031-9155. DOI: 10.1088/0031-9155/ $50 / 18 / 001$.

[49] T. Yilmaz, R. Foster, and Y. Hao, "Broadband Tissue Mimicking Phantoms and a Patch Resonator for Evaluating Noninvasive Monitoring of Blood Glucose Levels", IEEE Transactions on Antennas and Propagation, vol. 62, no. 6, pp. 3064-3075, Jun. 2014, ISSN: 0018926X. DOI: 10.1109/TAP . 2014. 2313139 . 
[50] P. A. Floor, R. Chávez-Santiago, S. Brovoll, Aardal, J. Bergsland, O. J.H. N. Grymyr, P. S. Halvorsen, R. Palomar, D. Plettemeier, S. E. Hamran, T. A. Ramstad, and I. Balasingham, "In-body to on-body ultrawideband propagation model derived from measurements in living animals", IEEE Journal of Biomedical and Health Informatics, vol. 19, no. 3, pp. 938948, 2015, ISSN: 2168-2194. DOI: 10.1109/JBHI .2015.2417805.

[51] C. Garcia-Pardo, R. Chavez-Santiago, N. Cardona, and I. Balasingham, "Experimental UWB frequency analysis for implant communications", in 2015 37th Annual International Conference of the IEEE Engineering in Medicine and Biology Society (EMBC), IEEE, Aug. 2015, pp. 54575460, ISBN: 978-1-4244-9271-8. DOI: 10.1109/EMBC. 2015.7319626.

[52] H. Xie, V. Shamdasani, A. T. Fernandez, R. Peterson, M. Lachman, Y. Shi, J.-L. Robert, M. Urban, S. Chen, and J. Greenleaf, "Shear wave Dispersion Ultrasound Vibrometry (SDUV) on an ultrasound system: In vivo measurement of liver viscoelasticity in healthy animals", in 2010 IEEE International Ultrasonics Symposium, San Diego, CA: IEEE, Oct. 2010, pp. 912-915, ISBN: 978-1-4577-0382-9. DOI: 10 . 1109 / ULTSYM . 2010.5935956.

[53] C. Garcia-Pardo, C. Andreu, A. Fornes-Leal, S. Castelló-Palacios, S. Perez-Simbor, M. Barbi, A. Vallés-Lluch, and N. Cardona, "Ultrawideband technology for medical in-body sensor networks: An overview of the human body as a propagation medium, phantoms, and approaches for propagation analysis", IEEE Antennas and Propagation Magazine, vol. 60 , no. 3 , pp. 19-33, 2018, ISSN: 1045-9243. DOI: 10.1109 /MAP . 2018.2818458.

[54] N. Cardona, S. Castello Palacios, A. Fornes Leal, C. Garcia Pardo, and A. Valles Lluch, Synthetic model of biological tissues for evaluating the wireless transmission of electromagnetic waves, Spanish Patent PCT/ES2016/070912, Jun. 2017.

[55] 3d guidance tracking system, http : //www . ndigital . com, Accessed: 2018-02-15.

[56] Xyz linear positioners and step motor systems, http://www arrickrobotics . com, Accessed: 2018-02-15. 
[57] M. Kawasaki and R. Kohno, "A toa based positioning technique of medical implanted devices", in 3rd International Symposium on Medical Information and communication Technology (ISMICT), Montreal: IEEE, 2008 .

[58] Pig anatomy, https : //www . biologycorner.com/pig/review.html, Accessed: 2018-04-20.

[59] X. Li, Body Matched Antennas for Microwave Medical Applications. KIT Scientific Publishing, 2014, ISBN: 9783731501473.

[60] C. A. Balanis, Antenna Theory: Analysis and Design, 3rd. New York: John Wiley \& Sons, 2005.

[61] Estudio, diseño y optimización de antena UWB para comunicaciones intracorporales, http : / / hdl . handle . net/10251/80429, Accessed: 2018-04-17.

[62] K. Y. Yazdandoost and R. Miura, "Miniaturized UWB implantable Antenna for Brain-Machine-Interface", in 2015 9th European Conference on Antennas and Propagation (EuCAP), Lisbon, 2015, pp. 1-5.

[63] Z. Bao, Y. X. Guo, and R. Mittra, "An ultrawideband conformal capsule antenna with stable impedance matching", IEEE Transactions on Antennas and Propagation, vol. 65, no. 10, pp. 5086-5094, Oct. 2017, ISSN: 0018-926X. DOI: 10.1109/TAP.2017.2741027.

[64] Y. Morimoto, D. Anzai, and J. Wang, "Design of ultra wide-band lowband implant antennas for capsule endoscope application", in 2013 7th International Symposium on Medical Information and Communication Technology (ISMICT), 2013, pp. 61-65. DOI: 10.1109/ISMICT . 2013. 6521700 .

[65] E. Miralles, C. Andreu, M. Cabedo-Fabres, M. Ferrando-Bataller, and J. F. Monserrat, "UWB on-body slotted patch antennas for in-body communications", in 2017 11th European Conference on Antennas and Propagation (EUCAP), IEEE, Mar. 2017, pp. 167-171, ISBN: 978-88907-0187-0. DOI: 10.23919/EuCAP. 2017.7928598. 
[66] Q. Wang, R. Hahnel, H. Zhang, and D. Plettemeier, "On-body directional antenna design for in-body UWB wireless communication", in 2012 6th European Conference on Antennas and Propagation (EUCAP), IEEE, Mar. 2012, pp. 1011-1015, ISBN: 978-1-4577-0920-3. DOI: 10.1109/EuCAP. 2012.6206211.

[67] X. Yu, J. Zhang, M. Haenggi, and K. B. Letaief, "Coverage analysis for millimeter wave networks: The impact of directional antenna arrays", IEEE Journal on Selected Areas in Communications, vol. 35, no. 7, pp. 1498-1512, 2017, ISSN: 0733-8716. DOI: 10.1109/JSAC. 2017. 2699098.

[68] L. A. Y. Poffelie, P. J. Soh, S. Yan, and G. A. E. Vandenbosch, "A high-fidelity all-textile uwb antenna with low back radiation for off-body wban applications", IEEE Transactions on Antennas and Propagation, vol. 64, no. 2, pp. 757-760, Feb. 2016, ISSN: 0018-926X. DOI: 10.1109/ TAP . 2015.2510035.

[69] S. Kang and C. W. Jung, "Wearable fabric reconfigurable beam steering antenna for on/off-body communication system", in 2015 IEEE International Symposium on Antennas and Propagation USNC/URSI National Radio Science Meeting, 2015, pp. 1211-1212. DOI: 10.1109/APS. 2015. 7304994.

[70] W. Jeong and J. Choi, "A low profile ir-uwb antenna with conical radiation pattern for on-body communications", in 2015 IEEE International Symposium on Antennas and Propagation USNC/URSI National Radio Science Meeting, 2015, pp. 2023-2024. DOI: 10.1109/APS . 2015. 7305401.

[71] Y. Morimoto, D. Anzai, and J. Wang, "Design of ultra wide-band lowband implant antennas for capsule endoscope application", in 2013 7th International Symposium on Medical Information and Communication Technology (ISMICT), IEEE, Mar. 2013, pp. 61-65, ISBN: 978-1-46735772-2. DOI: 10.1109/ISMICT . 2013.6521700.

[72] P. Hall and Y. Hao, "Antennas and propagation for body-centric wireless communications", in, 2006, p. 258. 
[73] Y. Zhang and A. K. Brown, "The discone antenna in a bpsk directsequence indoor uwb communication system", IEEE Transactions on Microwave Theory and Techniques, vol. 54, no. 4, pp. 1675-1680, 2006, ISSN: 0018-9480. DOI: 10.1109/TMTT .2006.872049.

[74] M. Ghafari, M. Adjrad, and M. Ghavam, "A novel uwb discone antenna for biomedical applications", in 2013 European Modelling Symposium, Nov. 2013, pp. 127-131. DOI: 10.1109/EMS .2013.22.

[75] A. Singh, "SIMULATIVE ANALYSIS OF DISCONE ANTENNA FOR 2.44 GHZ REGIME USING ANTENNA MAGUS", vol. 2, no. 2, 2012.

[76] W. Yan, A.-x. Chen, and T.-h. Jiang, "Design of UHF miniature discone antenna", in Proceedings of the 9th International Symposium on Antennas, Propagation and EM Theory, IEEE, Nov. 2010, pp. 356-358, ISBN: 978-1-4244-6906-2. DOI: 10.1109/ISAPE.2010.5696474.

[77] A. Andújar, J. Anguera, and C. Puente, "A systematic method to design broadband matching networks", in Proceedings of the Fourth European Conference on Antennas and Propagation, 2010, pp. 1-5.

[78] J. Liang, L. Guo, C. C. Chiau, and X. Chen, "CPW-fed circular disc monopole antenna for UWB applications", in 2005 IEEE International Workshop on Antenna Technology Small Antennas and Novel Metamaterials ( $i W A T$ ), Singapore: IEEE, 2005, pp. 505-508, ISBN: 0-7803-88429. DOI: 10.1109/IWAT . 2005.1461127.

[79] C. Tarin, P. Marti, L. Traver, N. Cardona, J. A. Diaz, and E. Antonino, "UWB Channel Measurements for Hand-Portable Devices: A Comparative Study", in 2007 IEEE 18th International Symposium on Personal, Indoor and Mobile Radio Communications, Athens, 2007, pp. 1-5, ISBN: 1424411440. DOI: 10.1109/PIMRC. 2007.4394768.

[80] M. Klemm, I. Z. Kovcs, G. F. Pedersen, and G. Troster, "Novel small-size directional antenna for uwb wban/wpan applications", IEEE Transactions on Antennas and Propagation, vol. 53, no. 12, pp. 3884-3896, Dec. 2005, ISSN: 0018-926X. DOI: 10.1109/TAP.2005.859906.

[81] C. Marchais, G. L. Ray, L. Lecoq, and A. Sharaiha, "A stripline fed printed wide slot antenna for uwb applications", in 2006 12th Interna- 
tional Symposium on Antenna Technology and Applied Electromagnetics and Canadian Radio Sciences Conference, 2006, pp. 1-4.

[82] P. Ara, M. Heimlich, and E. Dutkiewicz, "Antenna performance for localization of capsule endoscope", in 2014 8th International Symposium on Medical Information and Communication Technology (ISMICT), 2014, pp. 1-5. DOI: 10.1109/ISMICT . 2014.6825222.

[83] E. Pancera, Xuyang Li, M. Jalilvand, T. Zwick, and W. Wiesbeck, UWB medical diagnostic: in-body transmission modeling and applications, 2011.

[84] S. D. Targonski, R. B. Waterhouse, and D. M. Pozar, "Design of wideband aperture-stacked patch microstrip antennas", IEEE Transactions on Antennas and Propagation, vol. 46, no. 9, pp. 1245-1251, Sep. 1998, ISSN: 0018-926X. DOI: 10.1109/8.719966.

[85] S. Gezici, Z. Tian, G. B. Giannakis, H. Kobayashi, A. F. Molisch, H. V. Poor, and Z. Sahinoglu, "Localization via ultra-wideband radios: a look at positioning aspects for future sensor networks", IEEE Signal Processing Magazine, vol. 22, no. 4, pp. 70-84, Jul. 2005, ISSN: 1053-5888. DOI: 10.1109/MSP. 2005. 1458289 .

[86] B. Moussakhani, J. Flam, S. Stoa, I. Balasingham, and T. Ramstad, "On localisation accuracy inside the human abdomen region", IET Wireless Sensor Systems, vol. 2, no. 1, pp. 9-15, Mar. 2012, ISSN: 20436386. DOI: 10.1049/iet-wss. 2011.0060.

[87] C. Garcia-Pardo, A. Fornes-Leal, N. Cardona, R. Chávez-Santiago, J. Bergsland, I. Balasingham, S. Brovoll, O. Aardal, S.-E. Hamran, and R. Palomar, "Experimental ultra wideband path loss models for implant communications", in 2016 IEEE 27th Annual International Symposium on Personal, Indoor, and Mobile Radio Communications (PIMRC), Valencia: IEEE, Sep. 2016, pp. 1-6, ISBN: 978-1-5090-3254-9. DOI: 10 . 1109/PIMRC . 2016.7794780.

[88] T. Tuovinen, T. Kumpuniemi, K. Y. Yazdandoost, M. Hamalainen, and J. Iinatti, "Effect of the antenna-human body distance on the antenna matching in UWB WBAN applications", in 2013 7th International Symposium on Medical Information and Communication Technology (IS- 
MICT), IEEE, Mar. 2013, pp. 193-197, ISBN: 978-1-4673-5772-2. DOI: 10.1109/ISMICT . 2013.6521727.

[89] M. Fink and C. Prada, "Reports on Progress in Physics", Rep. Prog. Phys, vol. 63, 2005.

[90] R. C. Qiu, C. Zhou, N. Guo, and J. Q. Zhang, "Time reversal with miso for ultrawideband communications: Experimental results", IEEE Antennas and Wireless Propagation Letters, vol. 5, pp. 269-273, 2006, ISSN: 1536-1225. DOI: 10.1109/LAWP. 2006.875888.

[91] R. J. Barton and H.-C. Wu, "Performance of Cooperative Time-Reversal Communication in a Mobile Wireless Environment Performance of Cooperative Time-Reversal Communication", International Journal of Distributed Sensor Networks International Journal of Distributed Sensor Networks International Journal of Distributed Sensor Networks, vol. 3, no. 1 , pp. 59-68, 2007, ISSN: 1550-1329. DOI: 10.1080/15501320601067915.

[92] P. Pajusco and P. Pagani, "On the Use of Uniform Circular Arrays for Characterizing UWB Time Reversal", IEEE Transactions on Antennas and Propagation, vol. 57, no. 1, pp. 102-109, Jan. 2009, ISSN: 0018-926X. DOI: $10.1109 /$ TAP . 2008.2009715.

[93] Ning Kong and L. Milstein, "Average SNR of a generalized diversity selection combining scheme", IEEE Communications Letters, vol. 3, no. 3, pp. 57-59, Mar. 1999, ISSN: 1089-7798. DOI: 10.1109/4234.752901.

[94] S. Roy and P. Fortier, "Maximal-ratio combining architectures and performance with channel estimation based on a training sequence", IEEE Transactions on Wireless Communications, vol. 3, no. 4, pp. 1154-1164, 2004, ISSN: 1536-1276. DOI: 10.1109/TWC. 2004.828022.

[95] B. H. Fleury, M. Tschudin, R. Heddergott, D. Dahlhaus, and K. I. Pedersen, "Channel parameter estimation in mobile radio environments using the sage algorithm", IEEE Journal on Selected Areas in Communications, vol. 17 , no. 3, pp. 434-450, Mar. 1999, ISSN: 0733-8716. DOI: 10.1109/49.753729.

[96] R. J. M. Cramer, R. A. Scholtz, and M. Z. Win, "Evaluation of an ultrawide-band propagation channel", IEEE Transactions on Antennas and 
Propagation, vol. 50, no. 5, pp. 561-570, 2002, ISSN: 0018-926X. DOI: 10.1109/TAP. 2002 . 1011221.

[97] A. Khaleghi, R. Chávez-Santiago, and I. Balasingham, "Ultra-wideband statistical propagation channel model for implant sensors in the human chest", en, IET Microwaves, Antennas \& Propagation, vol. 5, no. 15, pp. 1805-1812, Dec. 2011, ISSN: 17518725. DOI: 10 . 1049 / iet-map . 2010.0537.

[98] L. Farrugia, P. S. Wismayer, L. Z. Mangion, and C. V. Sammut, "Accurate $<\mathrm{i}>$ in vivo $</ \mathrm{i}>$ dielectric properties of liver from $500 \mathrm{MHz}$ to 40 $\mathrm{GHz}$ and their correlation to $<\mathrm{i}>$ ex vivo $</ \mathrm{i}>$ measurements", Electromagnetic Biology and Medicine, vol. 35, no. 4, pp. 365-373, Oct. 2016, ISSN: $1536-8378$. DOI: $10.3109 / 15368378.2015 .1120221$.

[99] A. A. Bakar, A. Abbosh, and M. Bialkowski, "Fabrication and Characterization of a Heterogeneous Breast Phantom for Testing an Ultrawideband Microwave Imaging System", in Asia-Pacific Microwave Conference 2011, 2011, pp. 1414-1417, ISBN: 9780858259744.

[100] S Gabriel, R. W. Lau, and C Gabriel, "The dielectric properties of biological tissues: II. Measurements in the frequency range $10 \mathrm{~Hz}$ to 20 GHz", Physics in medicine and biology, vol. 41, pp. 2251-2269, 1996, ISSN: 0031-9155. DOI: $10.1088 / 0031-9155 / 41 / 11 / 002$.

[101] A. Abu Bakar, A. Abbosh, P. Sharpe, M. E. Bialkowski, and Y. Wang, "Heterogeneous Breast Phantom for Ultra Wideband Microwave Imaging", Microwave and Optical Technology Letters, vol. 53, no. 7, pp. 15951598, Jul. 2011. DOI: 10.1002/mop. arXiv: 0604155 [physics].

[102] A. Nyshadham, C. Sibbald, and S. Stuchly, "Permittivity measurements using open-ended sensors and reference liquid calibration-an uncertainty analysis", IEEE Transactions on Microwave Theory and Techniques, vol. 40, no. 2, pp. 305-314, 1992, ISSN: 00189480. DOI: $10.1109 / 22$. 120103.

[103] A. Fornes-Leal, C. Garcia-Pardo, N. Cardona, S. Sergio Castello-Palacios, and A. Valles-Lluch, "Accurate broadband measurement of electromagnetic tissue phantoms using open-ended coaxial systems", in 2017 11th International Symposium on Medical Information and Communication 
Technology (ISMICT), Lisbon: IEEE, Feb. 2017, pp. 32-36, ISBN: 9781-5090-5464-0. DOI: 10.1109/ISMICT . 2017.7891761.

[104] A. T. Mobashsher and A. M. Abbosh, "Artificial Human Phantoms: Human Proxy in Testing Microwave Apparatuses That Have Electromagnetic Interaction with the Human Body", IEEE Microwave Magazine, vol. 16, no. 6, pp. 42-62, Jul. 2015.

[105] S. Castelló-Palacios, C. Garcia-Pardo, A. Fornes-Leal, N. Cardona, and A. Vallés-Lluch, "Formulas for Easy-To-Prepare Tailored Phantoms at 2.4 GHz ISM Band", in 2017 11th International Symposium on Medical Information and Communication Technology (ISMICT), Lisbon, 2017, pp. $27-31$.

[106] C.-K. Chou, G.-W. Chen, A. W. Guy, and K. H. Luk, "Formulas for preparing phantom muscle tissue at various radiofrequencies", Bioelectromagnetics, vol. 5, no. 4, pp. 435-441, 1984, ISSN: 0197-8462. DOI: $10.1002 /$ bem . 2250050408 .

[107] S. Castelló-Palacios, C. Garcia-Pardo, A. Fornes-Leal, N. Cardona, and A. Vallés-Lluch, "Tailor-Made Tissue Phantoms Based on Acetonitrile Solutions for Microwave Applications up to 18 GHz", IEEE Transactions on Microwave Theory and Techniques, vol. 64, no. 11, pp. 3987-3994, Nov. 2016. 
